\title{
Fracture Propagation and Permeability Change under Poro-thermoelastic Loads \& Silica Reactivity in Enhanced Geothermal Systems DE-FG36-06G016059
}

\author{
Submitted by \\ Ahmad Ghassemi \\ Department of Petroleum Engineering \\ Texas A\&M University \\ College Station TX \\ October, 2009
}




\section{Table of Contents}

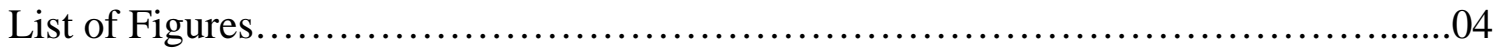

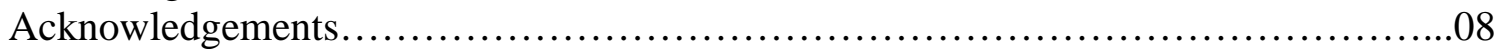

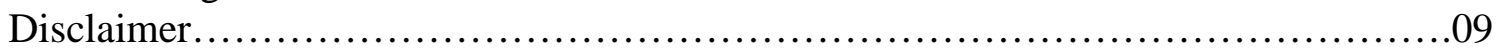

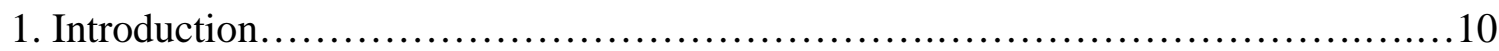

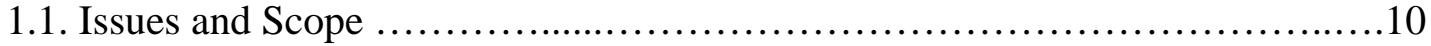

2. Fundamentals of Fracture Propagation \& Hydraulic Fracturing in Geothermal

Reservoirs: Concepts \& Mathematical Framework.............................13

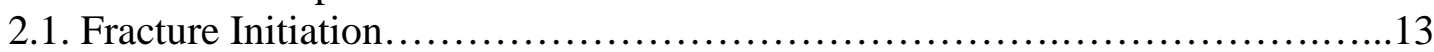

2.1.1. Hydraulic fracture initiation......................................... 16

2.2. Fracture Propagation................................................... 17

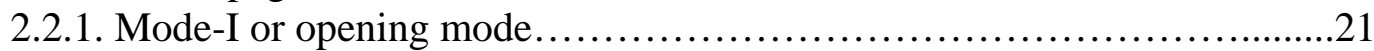

2.2.2. Mode-II or sliding mode..............................................21

2.2.3. Mode-III or tearing mode.......................................22

2.3. Fracture Propagation Criteria for Combined Loading........................25

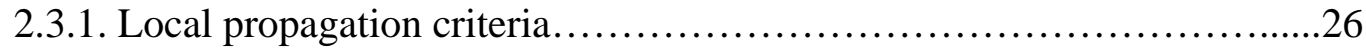

2.3.2. Global propagation criteria.........................................26

2.3.3. Structural (non-local) criteria.....................................26

2.4. A Unified Criterion for Mode I and II Propagation and Coalescence ............27

2.4.1. Mode \& direction of crack propagation............................29

3. Coupled Poro-thermoelastic Processes.........................................37

3.1. Governing Equations of Poro-thermoelasticity.............................. 37

3.1.1. Constitutive equations...............................................38

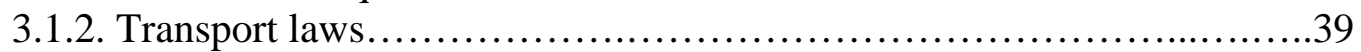

3.1.3. Balance laws......................................................40

3.2. Field Equation of Poro-thermoelaticity.....................................40

3.2.1. Field equation of poroelasticity and thermoelasticity..................41

3.3. Poro-thermoelastic Boundary Element Methods............................41

3.3.1. Displacement discontinuity method for poro-thermoelasticity............43

3.3.2. Natural fracture deformation using poro-thermoelastic DD...............48

3.4. Poro-thermoelastic Fractured Rock Response to Injection......................50

3.4.1. Fluid Flow \& Heat Transfer.............................................50

3.5. Solution Method.....................................................51

4. 3D-dimensional BEM for Fracture Response \& Injection-Induced Stresses..........55

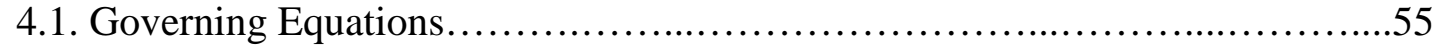

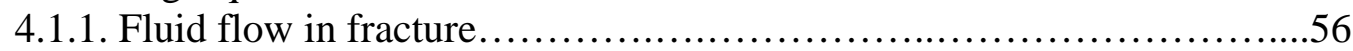

4.1.2. Fluid flow in reservoir matrix..................................... 57

4.1.3. Heat transport in fracture......................................57

4.1.4. Heat transport in reservoir......................................5 57

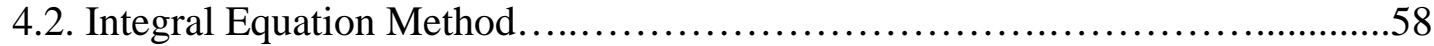

4.3. Treatment of Joint Deformation...........................................61

4.4. Finite Element Method for Fluid Flow \& Heat Transport in Fracture............64

4.5. Discretization of Integral Equations for Response of Reservoir Matrix..........65

4.6. Numerical Procedures for Flow \& Deformation in Natural Fracture..............70 


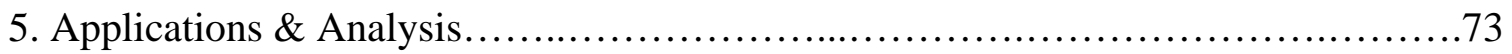

5.1. Poro-thermoelastic Analysis of Fracture Opening...............................73

5.1.1. Improved solution for short \& long time crack response..................77

5.2. Modeling Fracture Propagation ................................................

5.2.1. Propagation of multiple pressurized cracks................................. 83

5.3. Response Injection/Extraction into a Fractured Reservoir........................85

5.4. Three-Dimensional Poroelastic Analysis of Natural Fractures.....................89

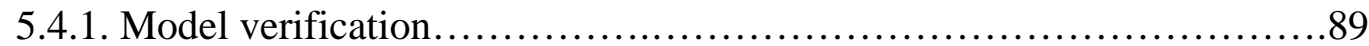

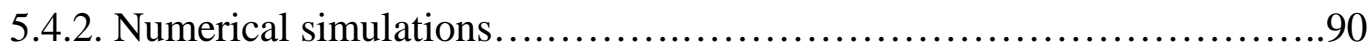

5.5. Three-Dimensional Analysis of Natural Fracture Response to Injection and

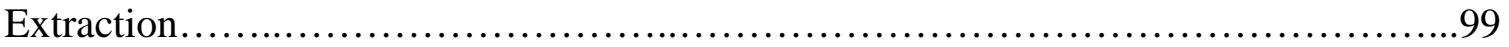

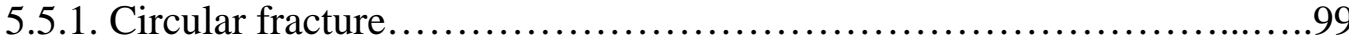

5.5.2. Irregularly-shaped fracture........................................113

6. Porothermoelastic Analysis of Reactive Flow in a Natural Fracture........................121

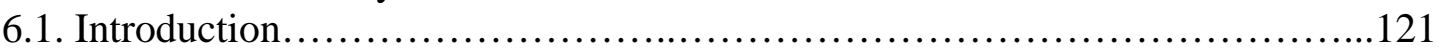

6.2. Modeling Approach \& Mathematical Model .....................................121

6.2.1. Fluid flow in the rock fracture......................................124

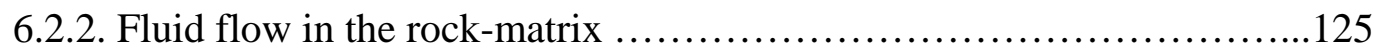

6.2.3. Heat transfer in the fracture and rock-matrix .........................125

6.2.4. Solute transport and reactivity.........................................126

6.3. Aperture Change Due to Silica Reactivity \& Poro- thermoelastic Effects.......127

6.3.1. Silica dissolution and precipitation and aperture variation.................127

6.3.2. Aperture variation caused by poro-thermoelasticity............................129

6.4. Temperature, Concentration, Pore Pressure \& Aperture Solution with Constant Leakoff (Permeable Rock-Matrix).............................................130

6.4.1. Temperature solution and thermoelastic effects on fracture aperture........131

6.4.2. Solution for silica concentration.......................................132

6.4.3. Pore pressure solution \& poroelastic effects on fracture aperture...........133

6.5. Temperature, Concentration, \& Aperture Solution (Impermeable Rock).........133

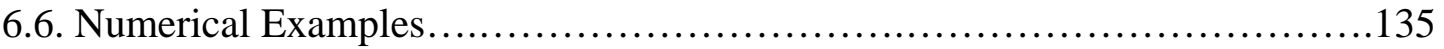

7. Conclusion \& Future Research.................................................. 145

7.1. Flow in Fractured Reservoir, Fracture Propagation, Permeability Change..145

7.2. Three-Dimensional Thermoelastic Effects.................................146

7.3. Influence of Quartz Precipitation/Dissolution on Fracture Permeability....148

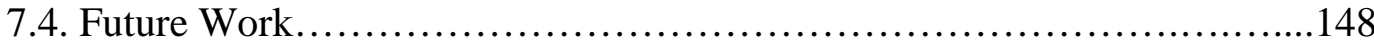

8. Publications \& Technology Transfer/ Student Training.............................149 


\section{List of Figures}

Figure 2.1. Stress state near the tip of a crack.

Figure 2.2. A sketch of three fundamental modes of fracture.

Figure 2.3. Geometry of an inclined crack.

Figure 2.4. Crack-tip closure resulting from Barenblatt's $3^{\text {rd }}$ postulate.

Fig. 2.5. Graphical interpretation of the edge and structural criteria. Edge criterion: the stress is calculated at a point $B$ at a distance equal to the length $d$ of the FPZ; Structural criterion: average stress over the segment $A B,|A B|=d$.

Figure 3.1. Illustration of coupling between poro-thermo-mechanical processes.

Figure 3.2. Discretization in the boundary element method.

Figure 3.3. Constant normal and shear displacement discontinuity.

Figure 3.4. Time marching scheme for a continuous heat/fluid source or DD.

Figure 3.5. A fluid filled fracture subject to normal and shear stress

Figure 4.1. Cartoon of injection/extraction heat extraction in a planar fracture.

Figure 4.2. Mohr-Coulomb model used for joint elements.

Figure 5.1. A uniformly pressurized, cooled crack.

Figure 5.2. Magnitude of crack opening due to an applied stress.

Figure 5.3. Normalized crack opening due to pore pressure loading.

Figure 5.4. Maximum crack opening due to cooling, also shown is the steady-state analytical value.

Figure 5.5. Crack opening for 3 loading modes using the improved DD method. Note the smooth transition of $\mathrm{DD}$ to a small time of $\mathrm{t}=0.01 \mathrm{sec}$. Crack opening is shown as negative.

Figure 5.6. Comparison of drained and un-drained solutions with analytical results.

Figure 5.7. Fracture opening caused by combined loading modes. Note the dominance of mode 1 during short times.

Figure 5.8. A pressurized crack under biaxial stresses in an infinite poroelastic medium. 
Figure 5.9. Crack propagation path at different speeds $\left(\mathrm{S}_{\mathrm{H}}=1 \mathrm{MPa}, \mathrm{S}_{\mathrm{h}}=0.5 \mathrm{MPa}, \mathrm{P}=2.5 \mathrm{MPa}\right.$, $\left.\mathrm{c} / \sigma_{t}=1.5\right)$.

Figure 5.10. Crack propagation path at different speeds $\left(\mathrm{S}_{\mathrm{H}}=3 \mathrm{MPa}, \mathrm{S}_{\mathrm{h}}=0.5 \mathrm{MPa}, \mathrm{P}=2.5 \mathrm{MPa}\right.$, $\left.\mathrm{c} / \sigma_{t}=1.5\right)$.

Figure 5.11. Crack propagation path at different speeds $\left(\mathrm{S}_{\mathrm{H}}=3 \mathrm{MPa}, \mathrm{S}_{\mathrm{h}}=0.5 \mathrm{MPa}, \mathrm{P}=2.5 \mathrm{MPa}\right.$, $\mathrm{c} / \sigma_{t}=1.1$.

Figure 5.12. Crack propagation paths for two parallel cracks using different models $\left(\mathrm{S}_{\mathrm{H}}=3 \mathrm{MPa}\right.$, $\left.\mathrm{S}_{\mathrm{h}}=0.5 \mathrm{MPa}, \mathrm{P}=2.5 \mathrm{MPa}, \mathrm{c} / \sigma_{t}=1.1\right)$.

Figure 5.13. Crack propagation path near an inclined crack at different crack propagation speed $\left(\mathrm{S}_{\mathrm{H}}=1 \mathrm{MPa}, \mathrm{S}_{\mathrm{h}}=0.5 \mathrm{MPa}, \mathrm{P}=3.5 \mathrm{MPa}, \mathrm{c} / \sigma_{t}=1.1\right)$.

Figure 5.14. Bottomhole pressure variation with cooling.

Figure 5.15. Reservoir pressure distribution with cooling effect at one day.

Figure 5.16. Reservoir pressure distribution with cooling effect at one week.

Figure 5.17. Reservoir pressure distribution with cooling effect at one month.

Figure 5.18. Reservoir pressure distribution with cooling effect at 1 year.

Figure 5.19. Mesh for a circular fracture used to verify the 3D numerical model.

Figure 5.20. Comparisons between numerical results and analytical results in the (a) normal and (b) shear direction.

Figure 5.21. Circular fracture mesh used in numerical example, where element $\mathrm{A}$ is at the center of the fracture.

Figure 5.22. Variations of fracture opening at element A: (a) Mode 1 and (b) Mode 2 loading.

Figure 5.23. Undrained and drained fracture opening profile in response to combined mode 1 and 2 loading, where $r$ is the radius of the computational point and $a$ is fracture radius.

Figure 5.24. Discretization of an irregularly shaped fracture by using 1834 four-noded quadrilateral elements.

Figure 5.25. Distribution of shear displacement component $D_{\mathrm{zx}}$ on the fracture.

Figure 5.26. Distribution of shear displacement component $D_{\text {zy }}$ on the fracture.

Figure 5.27. Distribution of normal displacement $D_{\mathrm{zz}}$ on the fracture.

Figure 5.28. Variation of fracture aperture $d_{n}$ for element B with time. 
Figure 5.29. Variation of slip in x-direction $d_{s x}$ for element B with time.

Figure 5.30. Variation of slip in y-direction $d_{s y}$ for element B with time.

Figure 5.31. Variation of normal effective stress $\sigma_{z z}^{\prime}$ for element B with time.

Figure 5.32. Fluid circulation in geothermal reservoir.

Figure 5.33. Discretization of a circular planar fracture: A is the injection well and B is the extraction well.

Figure 5.34. Distributions of poroelastic stress $\sigma_{\mathrm{zz}}$ from poro-thermoelastic model after different injection times: (a) $\mathrm{t}=1.0 \times 10^{5} \mathrm{~s}$; (b) $\mathrm{t}=1.0 \times 10^{7} \mathrm{~s}$; (c) $\mathrm{t}=1.0 \times 10^{9} \mathrm{~s}$

Figure 5.35. Distributions of thermoelastic stress $\sigma_{\mathrm{zz}}$ from poro-thermoelastic model after different injection times: (a) $\mathrm{t}=1.0 \times 10^{5} \mathrm{~s}$; (b) $\mathrm{t}=1.0 \times 10^{7} \mathrm{~s}$; (c) $\mathrm{t}=1.0 \times 10^{9} \mathrm{~s}$.

Figure 5.36. Distributions of the fracture aperture increment from poro-thermoelastic model after different injection times: (a) $\mathrm{t}=1.0 \times 10^{5} \mathrm{~s}$; (b) $\mathrm{t}=1.0 \times 10^{7} \mathrm{~s}$; (c) $\mathrm{t}=1.0 \times 10^{9} \mathrm{~s}$.

Figure 5.37. Distributions of fluid pressure in the fracture from poro-thermoelastic model after different injection times: (a) $\mathrm{t}=1.0 \times 10^{5} \mathrm{~s}$; (b) $\mathrm{t}=1.0 \times 10^{7} \mathrm{~s}$; (c) $\mathrm{t}=1.0 \times 10^{9} \mathrm{~s}$.

Figure 5.38. Distributions of fluid temperature in the fracture from poro-thermoelastic model after different injection times: (a) $\mathrm{t}=1.0 \times 10^{5} \mathrm{~s}$; (b) $\mathrm{t}=1.0 \times 10^{7} \mathrm{~s}$; (c) $\mathrm{t}=1.0 \times 10^{9} \mathrm{~s}$.

Figure 5.39. Comparison of fracture aperture increments at point A from the poroelastic, thermoelastic, and combined poro-thermoelastic models in the fluid injection and extraction process.

Figure 5.40. Comparison of fluid pressures at the location of point A from the poroelastic, thermoelastic, and poro-thermoelastic models during the fluid injection and extraction process.

Figure 5.41. Comparison of fracture aperture at points A, C, D and E during injection and extraction process from poroelastic, thermoelastic and combined models.

Figure 5.42. Comparison of fluid pressures at points A, C, D and E from the poroelastic, thermoelastic and combined models for injection and extraction process.

Figure 5.43. Discretization of an arbitrary shaped planar fracture, where A is fluid injection well, and $\mathrm{B}$ and $\mathrm{C}$ are fluid extraction wells.

Figure 5.44. Distributions of fluid temperature in the fracture from poro-thermoelastic model after different fluid injection times: (a) $\mathrm{t}=1.0 \times 105 \mathrm{~s}$; (b) $\mathrm{t}=1.0 \times 107 \mathrm{~s}$; (c) $\mathrm{t}=1.0 \times 109 \mathrm{~s}$. 
Figure 5.45. Distributions of fracture aperture increment after different fluid injection times: (a) $\mathrm{t}=1.0 \times 105 \mathrm{~s}$; (b) $\mathrm{t}=1.0 \times 107 \mathrm{~s}$; (c) $\mathrm{t}=1.0 \times 109 \mathrm{~s}$.

Figure 5.46. Distributions of fluid pressure in the fracture from poro-thermoelastic model after different fluid injection times: (a) $t=1.0 \times 10^{5} \mathrm{~s}$; (b) $\mathrm{t}=1.0 \times 10^{7} \mathrm{~s}$; (c) $\mathrm{t}=1.0 \times 10^{9} \mathrm{~s}$.

Figure 6.1. Idealized fracture and rock- matrix system.

Figure 6.2. Normalized fracture aperture due to silica reactivity for different injection times (no leak-off).

Figure 6.3. Concentration distribution in the fracture after 1 week, 1 month and 3 months when pure water is injected in the fracture.

Figure 6.4. Temperature distribution in the fracture after 1 week, 1 month and 3 months.

Figure 6.5. Normalized pressure profile in the fracture after 1 week, 1 month, 3 months.

Figure 6.6. Normalized fracture aperture due to silica dissolution/precipitation at 1 week and 3 months (undersaturated case; Solid lines: No leak-off: and symbols: $\mathrm{m}=0.5$ ).

Figure 6.7. Normalized fracture aperture due to silica dissolution/precipitation at 1 week and 3 months (super-saturated case; Solid lines: No leak-off: and symbols: $\mathrm{m}=0.5$ ).

Figure 6.8. Normalized fracture aperture due to influence of poroelastic stress on fracture aperture after 3 months. The figure is drawn for different fluid loss/injection ratios (m).

Figure 6.9. Normalized fracture aperture at the injection point due to influence of poroelastic stress on fracture aperture for different fluid loss/injection ratios (m) and injection times.

Figure 6.10. Normalized fracture aperture at the injection point due to thermoelastic effects for different fluid loss/injection ratios (m) and injection times.

Figure 6.11. Normalized pressure profile along the fracture for different fluid loss/injection ratios (m) after 3 months of injection. Lines: poroelastic case and symbols: thermoelastic case. 


\section{ACKNOWLEDGEMENTS}

The PI (A. Ghassemi) has benefited from the contributions of many individuals in completing this project, including graduate students, post-doctoral fellows, and consultants; whose efforts are reflected in the publications listed at the end of this report. Chakra Rawal, Jun Ge, Qingfen Tao, and Wenxu Xue worked on various aspects of the project for their Ph.D. and M.S. theses. Xiaixian Zhao and Zhennan Zhang participated in this work as Pos-Docs and contributed to the fracture modeling and 3D model developments. Prof. A. Cheng took part in formulation of the 2D heat extraction/chemical transport problem; the research also benefited from his consultations in various solution strategies of the $3 \mathrm{D}$ integral equations. 


\section{DISCLAIMER}

This information was prepared as an account of work sponsored by an agency of the U.S. Government. Neither the U.S. Government nor any agency thereof, nor any of their employees, makes any warranty, express or implied, or assumes any legal liability or responsibility for the accuracy, completeness, or usefulness of any information, apparatus, product, or process disclosed, or represents that its use would not infringe privately owned rights. References herein to any specific commercial product, process, or service by trade name, trademark, manufacturer, or otherwise, does not necessarily constitute or imply its endorsement, recommendation, or favoring by the U.S. Government or any agency thereof. The views and opinions of authors expressed herein do not necessarily state or reflect those of the U.S. Government or any agency thereof. 


\section{Introduction}

Geothermal energy is recovered by circulating water through heat exchange areas within a hot rock mass. Geothermal reservoir rock masses generally consist of igneous and metamorphic rocks that have low matrix permeability. Therefore, cracks and fractures play a significant role in extraction of geothermal energy by providing the major pathways for fluid flow and heat exchange. Therefore, knowledge of the conditions leading to formation of fractures and fracture networks is of paramount importance. Furthermore, in the absence of natural fractures or adequate connectivity, artificial fractures are created in the reservoir using hydraulic fracturing. Multiple fractures are preferred because of the large size necessary when using only a single fracture. Although the basic idea is rather simple, hydraulic fracturing is a complex process involving interactions of high pressure fluid injections with a stressed hot rock mass, mechanical interaction of induced fractures with existing natural fractures, and the spatial and temporal variations of in-situ stress. As a result, it is necessary to develop tools that can be used to study these interactions as an integral part of a comprehensive approach to geothermal reservoir development, particularly enhanced geothermal systems. In response to this need we have developed advanced poro-thermo-chemo-mechanical fracture models for rock fracture research in support of EGS design.

The fracture propagation models are based on a regular displacement discontinuity formulation. The fracture propagation studies include modeling interaction of induced fractures. In addition to the fracture propagation studies, two-dimensional solution algorithms have been developed and used to estimate the impact of pro-thermo-chemical processes on fracture permeability and reservoir pressure. Fracture permeability variation is studied using a coupled thermo-chemical model with quartz reaction kinetics. The model is applied to study quartz precipitation/dissolution, as well as the variation in fracture aperture and pressure. Also, a three-dimensional model of injection/extraction has been developed to consider the impact poro- and thermoelastic stresses on fracture slip and injection pressure. These investigations shed light on the processes involved in the observed phenomenon of injection pressure variation (e.g., in Coso), and allow the assessment of the potential of thermal and chemical stimulation strategies.

\subsection{Issues \& Scope}

Fracture propagation and permeability enhancement are very important in development of geothermal reservoirs, particularly EGS. Important coupled processes that control flow and heat extraction in a geothermal reservoir include (i) fracture closure/opening in response to changing effective normal stress, (ii) fracture shear dilation during stimulation and circulation, (iii) poro-thermoelastic effects in stimulation and circulation operations, and (iv) chemical dissolution and precipitation during circulation.

The purpose of the project was to investigate fracture response to water injection, and to study the variation of fracture permeability and fluid pressure near injection regions of 
EGS while considering coupled poro-thermoelastic effects and mineral precipitation/dissolution processes. Part (I) of the project involved development of 2D models to study mode I and II fracture propagation and interaction as well as fracture opening and slip in geothermal reservoirs using the boundary element method; and development of a three-dimensional poro-thermoelastic fracture models to study the impact of injection/extraction on the reservoir's state of stress and fracture slip with reference to Coso injection experiments. Part (II) consisted of the development of fundamental analytical and numerical models to study the individual influences of thermal, poroelastic, and silica dissolution/precipitation processes on the fracture permeability and pressure variations over temporal and spatial scales of interest to reservoir development and injection/extraction operations. The project has resulted in the completion of the following:

1. development of a 2D poro-thermoelastic model for analysis of injection/extraction in fractured rock

2. development of a two-dimensional poroelastic boundary element code for modeling single/multiple fracture propagation and coalescence with natural discontinuity such as joints and faults

3. 2D modeling of permeability change caused by poro-thermo-chemical processes (silica precipitation/dissolution)

4. Three-dimensional modeling of variation of in-situ stress due to injection/extraction based on three-dimensional modeling of injection induced thermal stresses

By considering the relevant rock mechanics issues and processes in fracturing and natural fracture behavior, the project has advanced the state-of-the-knowledge and technology in the high pressure thermo-mechanical rock/fluid interactions. It is anticipated that utilizing the project outcomes will improve the technology for designing artificial fractures and enhanced geothermal systems.

The body of this report consists of two parts. Part I deals with rock failure and fracture propagation and poro-thermoelastic effects on fracture permeability and fluid flow in fractured rock; it includes Chapters 1-5. Part II pertains to geochemistry and the impact of silica reactivity on fracture permeability and fluid flow; it consists of Chapter 6. The fundamental concepts and theoretical issues involved in rock failure and fracture propagation, thermoelasticity, poro-thermoelasticity, and details of the numerical boundary element methods are described in Chapters 1-4. Chapter 5 is devoted to application and analysis; it includes model applications to problems of interest such as fracture opening and slip, analysis of injection/production in naturally fractured reservoir, and fracture propagation. In addition, examples of 3D analysis of fracture slip are presented. Chapter 6 describes theoretical and analytical/numerical aspects of reactive flow in fractures. In addition, example applications are presented to illustrate the 
combined effects of silica dissolution and precipitation and poro-thermoelastic processes on fracture aperture and flow and pressure distribution in a fracture during circulation operations. The report ends with conclusions and practical guidelines as well as a few recommendations for future work. 


\section{Fundamentals of Fracture Propagation \& Hydraulic Fracturing in Geothermal Reservoirs: Concepts \& Mathematical Framework}

Hydraulic fracturing and stimulation of geothermal reservoirs involves a number of processes such as fracture initiation, fracture propagation, fluid flow in a deformable fracture, fluid diffusion into the rock, and heat transfer between the fluid and rock. Each of these aspects forms, in itself, a complex problem which has been the subject of many investigations and merits further study. A detailed examination of these processes with the intent of simultaneously improving their treatment is beyond the objectives of the present investigation. However, it is beneficial to review fracture initiation and propagation as they are very important in understanding the dynamics of fractures in geothermal fields and reservoir development.

During pressurization of the borehole, the solid (rock) is subjected to a system of external loads under certain environmental conditions (stress, temperature, chemical state). The response of the rock to imposed loads is simply one of deformation in the sense that the rock remains topologically unchanged [1]. Application of the load will eventually cause the formation of a fracture creating new surface areas within the rock mass. Therefore, from a mathematical viewpoint, two types of problems may be distinguished: fracture initiation and fracture propagation.

\subsection{Fracture Initiation}

In dealing with the fracture initiation problem, one needs to evaluate the critical level of the applied loads that correspond to inception of a fracture. An appropriate fracture criterion is necessary to augment the analysis of deformation and stresses, for the purpose of relating it to the fracture resistance of the rock. Several different failure criteria are in existence for use in various applications [2]:

- Maximum tensile stress theory; according to this theory, failure initiates when the minimum principal stress component, $\sigma_{3}$, reaches the tensile strength, $T_{0}$, of the material, $\sigma_{3}=-T_{0}$.

- Coulomb's theory; it is the maximum shear stress criterion which postulates that fracture will occur at a point in the material for a specific value $S_{0}$, referred to as the shear strength $\left[\left(\sigma_{1}-\sigma_{3}\right) / 2\right] \geq S_{0}$ with $\sigma_{1}$ and $\sigma_{3}$ representing the major and minor principal stresses, respectively. According to this theory the failure plane will bisect the angle between the minor and major principal stresses so that in a triaxial test, the plane of failure should be at $45^{\circ}$ to the axial stress. This conclusion, however, is not observed experimentally. The orientation of 
the failure plane varies with rock type and is less than $45^{\circ}$ to the direction of maximum compression.

- Coulomb-Navier (Modified max. shear criterion); Coulomb's theory was modified by Navier to include the influence of the normal stress acting on the plane of failure which tends to increase the shear resistance of the material [3]. Representing the normal stress by $\sigma$ and the shear stress by $\tau$, the theory stipulates that failure occurs when the magnitude of the shear stress acting on the failure plane reaches a value given by $|\tau|=S_{0}+\mu^{*} \sigma$ in which $\mu^{*}$ is the coefficient of internal friction. This criterion for failure indicates that the angle between the failure plane and the direction of maximum load, $\theta$, will be less than $45^{\circ}$ which is in agreement with experimental evidence [3].

- Mohr's Theory; it postulates that a material fractures or begins to deform permanently when the shear stress acting on the plane of failure increases to a value which is a function of the normal stress acting on the same plane $\tau$ $=f(\sigma)$. However, if the largest tensile principal stress has reached the tensile strength of the material, the maximum tensile stress theory, mentioned previously, is assumed. The form of $f(\sigma)$ is determined experimentally. Unlike the previously described criteria, Mohr's theory of failure predicts the direction of failure in addition to the state of stress at which failure occurs. Mohr's theory also implies that the value of the intermediate stress does not affect the shear stress, $\tau$, so that failure is independent of this stress. It also indicates that failure will not occur in hydrostatic compression consistent with experimental observation. It should be mentioned that the Coulomb-Navier and Mohr criteria are identical if the coefficient of internal friction is constant [4].

- Griffith's Theory; in contrast to the previously described empirical criteria that do not relate failure to any internal mechanisms or processes taking place in the rock material, the [5] theory is based on a given mechanism and relates failure to it. According to Griffith, two conditions are necessary for fracture; a stress condition and an energy condition, i.e., the cohesive forces must be exceeded and the energy required to form two surfaces in the solid must be provided. Together, these conditions are sufficient to ensure fracture. The first condition provides the basis for a failure criterion, while many fracture propagation criteria originate from the energy condition. Griffith's theory is based on the assumption that rocks contain flaws such as microcracks. When the rock body is subjected to external loads, stress concentrations develop around these features. These stress concentrations cause crack initiation and growth, which will ultimately cause macroscopic failure. Griffith considered the problem of a thin, linearly elastic, isotropic, infinite plate, containing a thin elliptical slit of length 2a subjected to a tensile stress $\sigma$. The normal stress developed at the tip of the crack, due to the applied load, is given by [6]: 
$\sigma_{t}=\sigma\left(1+\frac{4 a}{2 b}\right)$

in which $2 \mathrm{a}$ and $2 \mathrm{~b}$ represent the major and minor axes of the elliptical hole. Thus for sharp slits (i.e. large values of $(a / b)$ the stress, $\sigma_{\mathrm{t}}$, could be large enough to cause failure at moderate levels of applied load. This formed the basis for Griffith's analysis and formulation of his stress condition for failure. [7] derived his failure criterion by considering the variation of the tangential stress $\sigma_{\mathrm{t}}$ at the surface of a flat elliptical crack under the action of twodimensional stresses $\sigma_{1}, \sigma_{2}$ at infinity and obtained:

$$
\frac{\left(\sigma_{1}-\sigma_{3}\right)^{2}}{\sigma_{1}+\sigma_{3}}=8 T_{0}
$$

If $\sigma_{3}=0$ and $\sigma_{1}=C \mathrm{o}$, the uniaxial compressive strength of the rock, the above relation indicates that $T_{0}=\left(C_{0}\right)$, i.e., tensile strength is exactly one eighth of the uniaxial compressive strength, a condition that is not consistent with experimental observations. The compressive strength of most rocks varies from 10 to 100 times the tensile strength [8]. In addition, [9] showed that the failure criterion corresponds to a Mohr's envelope at failure is given by:

$$
\tau^{2}+4 T_{0} \sigma=4 T_{0}^{2}
$$

This is a parabolic criterion, and so it is not valid for many rocks (especially igneous). Typically, brittle rocks, such as granite and quartzite for which the Griffith criterion is most likely valid, have a straight envelope in compression, in disagreement with the normal Griffith criterion [10]. When modified to include closing of cracks in compression [11], the prediction turns out to be linear and identical to the Navier-Coulomb criterion, upon complete closure of cracks. This modified version gives an improved ratio of the compressive-totensile strengths for rocks of 12 , but the ratio is still low in comparison with that obtained from measurements [10]. It should also be kept in mind that the Griffith theory does not predict the path of a growing crack except when loaded normal to its surface (simple tension). It does, however, indicate that, in compression, the direction in which fracture is initiated is not in the direction of the original crack and turns towards the direction of the maximum principal stress. However, Griffith's failure criterion is valid in both tension and compression. This is very important and adds to the attractiveness of the theory, particularly when viewed in light of the fact that tension and shear are the two basic modes causing fracture, and there is no fundamental compression mechanism of failure. Finally, similar to Coulomb and Mohr criteria; Griffith's theory predicts that the intermediate principal stress has no effect on the strength. 


\subsubsection{Hydraulic Fracture Initiation}

The maximum tensile stress theory is often used to predict the tensile failure of the borehole wall $\left(\sigma_{3}=-T_{0}\right)$ and hydraulic fracture initiation. For a borehole in an impermeable rock formation, whose axis is parallel to the major principal stress, the condition for failure and creation of a tensile fracture is given by:

$$
3 \sigma_{h, \text { min }}-\sigma_{H, \text { max }}-p+T=p_{b}
$$

in which $p$ is the initial pore pressure, and $p_{\mathrm{b}}$ is the breakdown pressure which is viewed as the pressure at which the crack is formed; often assumed to correspond to the initial peak of the pressure-time record. $T$ represents the tensile strength of the intact rock material, and $\mathrm{p}$ is the reservoir pore pressure. The term $\left(3 \sigma_{\mathrm{h}, \min }-\sigma_{\mathrm{H}, \max }\right)$ in the above equation represents the least stress concentration along the borehole wall due to the farfield horizontal stresses, and is obtained from the Kirsch solution [12].

However, there are indications that shear may be a mode of failure in initiation of hydraulic fractures from inclined wellbores [13,14,15]. Indeed, some investigators [16], [17] argue that a hydraulic fracture is induced by shear rather than tensile failure and use a Mohr-Coulomb shear failure criterion.

As noted by [18], neither the tensile nor the shear failure criterion is capable of predicting the high breakdown pressures observed in the laboratory as well as its dependence on borehole size and injection rate. A fracture mechanics model [19-23) based on the concept of unstable fracture propagation using the fracture toughness criterion, $K_{\mathrm{I}}=K_{\mathrm{c}}$, seems more promising. Introduction of an additional condition, namely, $\partial\left(K_{\mathrm{I}}-K_{\mathrm{c}}\right.$ )$/(\partial \mathrm{L}) \geq 0$ makes it possible to take into account phenomena such as pumping rate- and size-dependence of the breakdown pressure.

Fracture initiation from a wellbore may be treated using the classical strength of materials approach [24], with Eq. (2.4) or the fractures mechanics approach [19,22-23]. The fracture mechanics approach can be considered by using a fracture propagation model based on the principles of fracture mechanics. In some instances it provides a better estimate of the breakdown pressure. Also, the structural criterion [25] implemented in our numerical mode may be used to predict both initiation and propagation. However, because of its practicality, the classical approach is the one that is widely used. We will also adopt it in this work unless otherwise indicated for a particular case (as in Chapter 5 when estimating the $S_{H \max }$ magnitude).

The above Eq. (2.4) for fracture initiation pressure does not take into account the influence of poroelastic and thermoelastic effects. Using the classical approach, fracturing of a vertical well in a cooled rock was considered in [26] it was found that thermal stresses can impose a significant influence on hydraulic fracturing and interpretation of its results to determine the maximum far-field stress. Cooling the rock induces tensile stresses and results in a lower wellbore pressure for fracture initiation. Numerical and analytical models have been developed that can calculate the stress 
distribution around a wellbore of arbitrary orientation while considering, thermoelastic, and poroelastic effects. These are useful for calculating the failure pressure and its location around the wellbore based on the classical approach. The details of the governing equation and solution methodologies can be found in [27-28] for the analytical approach; and in (Ghassemi, and Zhang, 2004) for the numerical solution. Certain aspects of the latter appear in this report also.

Once the onset of failure and fracture initiation is established from the knowledge of the stresses and the limit of the material's ability to carry an applied load (i.e., a failure criterion), the question arises as to if and how the fracture propagates. This is the subject of fracture propagation modeling portion of this project. But, we note that it is possible in principle to apply this numerical model can to study fracture initiation using a fracture mechanics approach. This is not, however, pursued in this work.

\subsection{Fracture Propagation}

Whether a fracture subject to a system of loads propagates, and if the propagation is stable or not is the subject of the field of fracture mechanics. In this work fracture propagation is studied within the framework of what is known as the field of Linear Elastic Fracture Mechanics (LEFM), the mathematical framework of which is described in detail below. In situations where the fracture process zone is not small or when dealing with fracture intersection and coalescence, a new non-local criterion is proposed and used. This criterion in effect extends the LEFM for use in the case of a non-small FPZ and for cases when SIFs cannot not be used.

According to LEFM, near the crack tip the displacements are proportional to $ل_{\mathrm{r}}$ and the stresses contain a singularity of the form $1 / \sqrt{ }$. This modern theory of fracture mechanics has its roots in the work of Griffith [5] who emphasized the energy dissipated in an increment of fracture extension, which is now referred to as the energy release rate, $G$.

In the previous Section, Griffith's theory was described within the framework of a failure criterion. It was indicated that to initiate a fracture it is necessary to have a stress concentration which is provided by inherent microcracks or flaws. This stress condition is necessary but not sufficient for fracture propagation as very sharp flaws may be present within a loaded body without causing failure. Indeed, Inglis' [7] results show that the stresses at the edge of a sharp crack can tend to infinity. This paradox can be understood by the second aspect of Griffith's theory, namely the energy balance which leads to a critical condition for fracture propagation. It is written as an equality between the change in the potential energy due to an increment of crack extension and the energy required to create two new surfaces [29]:

$$
\frac{d(W-U)}{d A}=\gamma
$$

where $W$ and $U$ are the external work done on the body and its internal strain energy, respectively, and $A=4 B a$ is the crack surface area for an internal fracture of length $2 a$ in a 
body of thickness $B$ (it equals unity under plain strain condition). Westergaard's solution [30] for the opening displacement, $v$, of the Griffith crack is given by:

$$
v=\frac{2 \sigma}{E^{\prime}}\left(a^{2}-x^{2}\right)^{\frac{1}{2}}
$$

in which $E^{\prime}=E$ for plane stress, $E^{\prime}=\left(E /\left(1-v^{2}\right)\right)$ for plane strain, and $\sigma$ is the applied stress. Thus, it can be shown that:

$$
W-U=4 B \int_{0}^{a} \frac{1}{2} \sigma(x) d x=\frac{B \pi a^{2} \sigma^{2}}{E^{\prime}}
$$

Assuming fixed grip conditions (constant displacement at the boundary) so that the external work equals zero, and substituting Eq. (2.5) gives the strain energy released when the crack grows by an amount $d a$ :

$$
\frac{d U}{d a}=\frac{\pi a \sigma^{2}}{2 E^{\prime}}
$$

This can be solved to yield the magnitude of the stress at failure:

$$
\sigma=\left(\frac{2 E^{\prime} \gamma}{\pi a}\right)^{\frac{1}{2}}
$$

The strain energy release rate at one tip is:

$$
G=\frac{\pi \sigma^{2} a}{4 E^{\prime}}
$$

Therefore, Griffith's energy balance states that crack growth occurs when $G$ reaches a critical value, $G_{c}$; a material property representing its resistance to crack growth. The parameter $\mathrm{G}$ can be expressed in terms of tip parameters. This became possible as a result of the work of Westergaard who used stress functions derived from complex potentials to find expressions for distribution of stresses around the tip of cracks with thicknesses approaching zero. Sneddon [31] applied Westergaard's results to both two- and threedimensional pressurized cracks. The stress field in the vicinity of the tip of a twodimensional crack is given by:

$$
\left[\begin{array}{c}
\sigma_{x} \\
\sigma_{y} \\
\tau_{x y}
\end{array}\right]=P \sqrt{\frac{a}{2 r}}\left[\begin{array}{c}
\frac{3}{4} \cos \frac{\theta}{2}+\frac{1}{4} \cos \frac{5 \theta}{2} \\
\frac{5}{4} \cos \frac{\theta}{2}-\frac{1}{4} \cos \frac{5 \theta}{2} \\
\frac{1}{2} \sin \theta \cos \frac{3 \theta}{2}
\end{array}\right]
$$


and for a penny-shaped crack,

$$
\left[\begin{array}{c}
\sigma_{r} \\
\sigma_{z} \\
\tau_{z r}
\end{array}\right]=\frac{2 P}{\pi} \sqrt{\frac{a}{2 r}}\left[\begin{array}{c}
\frac{3}{4} \cos \frac{\theta}{2}+\frac{1}{4} \cos \frac{5 \theta}{2} \\
\frac{5}{4} \cos \frac{\theta}{2}-\frac{1}{4} \cos \frac{5 \theta}{2} \\
\frac{1}{2} \sin \theta \cos \frac{3 \theta}{2}
\end{array}\right]
$$

where $P$ is the internal pressure on the crack face, $a$ is the fracture half-length in twodimensions and its radius in three dimensions, $r$ is the distance from a point to the crack tip, and $\theta$ represents the angle that $r$ makes on the central plane of the crack (Figure 2.1).

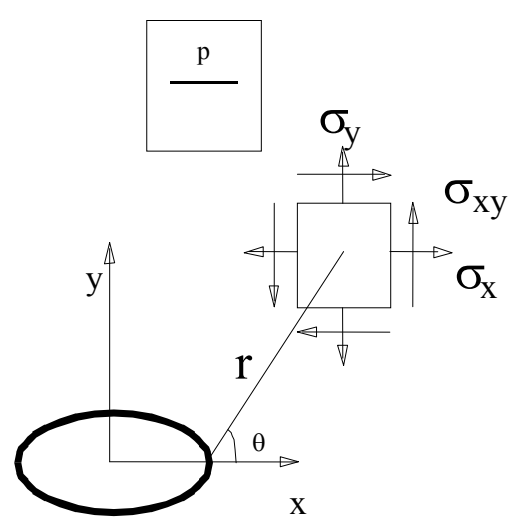

$2 \mathrm{c}$

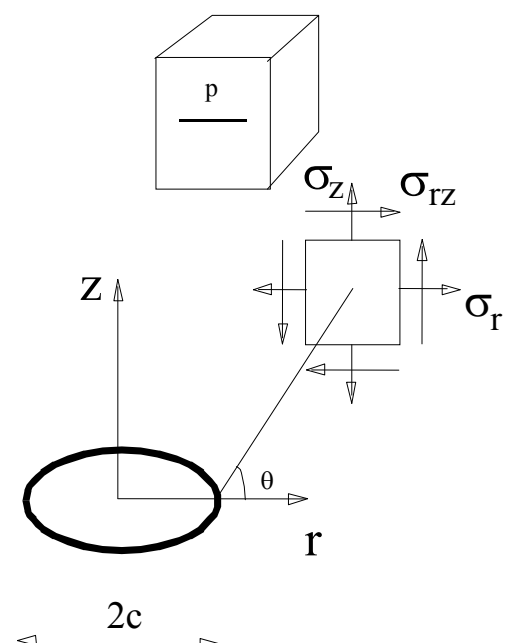

Figure 2.1. Stress state near the tip of a crack.

As can be observed, and noted by Sneddon [31] the above expressions for stress components around the tip in three dimensions differ from those in the two-dimensional case by a numerical factor only.

Writing the second of Eqs. (2.11) for the case $\theta=0$, and translating the center of the coordinate system to the center of the crack yields:

$$
\sigma_{y}=\frac{K}{\sqrt{2 \pi(x-a)}}
$$


where $K$ is the stress intensity factor. It is a quantity which depends on the geometry and loading and has the value $\sigma \sqrt{ }(2 \pi \mathrm{a})$ where $\sigma=\operatorname{limit}\left(\sigma_{\mathrm{y}}\right)$ as $x \rightarrow a$. The crack opening displacement in terms of $K$ is given by Kanninen and Popelar [29]:

$$
v=\frac{(1+v)(\kappa+1)}{E} K\left(\frac{a-x}{2 \pi}\right)^{\frac{1}{2}}
$$

in which $\kappa$ equals $(3-v) /(1+v)$ for plane stress and $(3-4 v)$ for plane strain, and $v$ represents the Poisson's ratio. Using these equations, Irwin [32] calculated the work required to close the crack that has extended by an amount $\Delta a$, which may be equated to the product of the energy release rate and the increment of crack extension. Therefore,

$$
G \Delta a=2 \int_{a}^{a+\Delta a} \frac{1}{2} \sigma_{y}(x) v(x-\Delta a) d x
$$

so that:

$$
G=\frac{K^{2}}{E^{\prime}}
$$

This expression, derived by Irwin [32], relating the Griffith's strain energy release rate, a global parameter, to the stress intensity factor, a local crack-tip quantity, is considered as a major contribution. It shows that Griffith's criterion for crack growth is essentially identical with that of crack growth when the stress intensity factor, K, reaches a critical value $K_{\mathrm{c}}$. This parameter is known as the fracture toughness. It is a material property which can be determined experimentally, and is known to be a function of temperature, confining pressure, and moisture content [33]. But, when defining the fracture toughness, $K_{\text {c }}$, it is assumed that the material is homogeneous and linearly elastic. These conditions do not generally hold true in the case of rock, and nonlinear behavior prevails at the crack tip in case of a homogeneous, linear elastic rock.

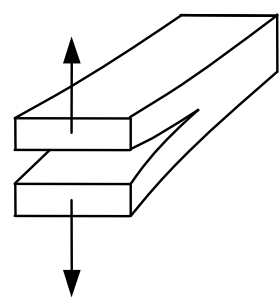

I

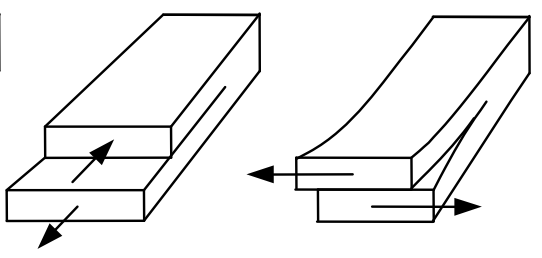

II

III

Figure 2.2. A sketch of three fundamental modes of fracture.

The size of this zone has a large influence on the value of the fracture toughness and results in a scale effect. Indeed, laboratory tests indicate that fracture toughness increases with an increase in crack length or specimen size [34]. For large crack lengths or 
specimens, $K_{\mathrm{c}}$ becomes independent of size, however, and approaches an asymptotic value considered to be an intrinsic material parameter called fracture toughness. When applied to a hydraulic fracture, the above criterion predicts that the higher the fracture toughness, the higher the pressure required to extend the fracture, and the longer the fracture, the lower the pressure required for its extension (if the overburden remains constant).

Hydraulically-driven fractures are usually considered to propagate in Mode I or the opening mode, however, mix-mode propagation is possible and will be considered in the modeling efforts. It is useful to review other modes of fracture propagation as shear growth may take place on a macro-level in some geologic situations, out-of-plane propagation, and injection into pre-existing joints in geothermal reservoirs.

In addition to a crack loaded in uniaxial tension, Irwin also considered other configurations of fracture loading and classified them into three independent modes. Mode-I is the above considered problem, Mode-II refers to a crack loaded in shear, i.e., the load is tangential to the crack surface in the direction of propagation; and Mode-III is defined by a crack loaded by a tangential load in the direction perpendicular to the direction of propagation. This is also called the anti-plane mode (Figure 2.2). The equations for stresses and displacement for these problems are as given below:

\subsubsection{Mode-I or opening mode}

In this mode, the displacements of the crack surfaces are perpendicular to the plane of the crack. Mode-I crack tip stress and displacement fields are [32]:

$$
\begin{gathered}
{\left[\begin{array}{c}
\sigma_{r} \\
\sigma_{\theta} \\
\tau_{r \theta}
\end{array}\right]=\frac{K_{I}}{\sqrt{2 \pi r}} \cos 2 \theta\left[\begin{array}{c}
1+\sin ^{2} \frac{\theta}{2} \\
\cos ^{2} \frac{\theta}{2} \\
\sin \frac{\theta}{2} \cos \frac{\theta}{2}
\end{array}\right]} \\
\sigma_{z}=v\left(\sigma_{r}+\sigma_{\theta}\right) \\
\sigma_{z}=\tau_{r z}=\tau_{\theta z}=0 \\
\tau_{r z}=\tau_{r \theta}=0 \\
{\left[\begin{array}{c}
u \\
v \\
w
\end{array}\right]=\frac{K_{I}}{4 G} \sqrt{\frac{r}{2 \pi}}\left[\begin{array}{c}
2(\kappa-1) \cos \frac{\theta}{2}-\cos \frac{3 \theta}{2} \\
-2(\kappa-1) \sin \frac{\theta}{2}+\sin \frac{3 \theta}{2} \\
0
\end{array}\right]}
\end{gathered}
$$

\subsubsection{Mode-II or sliding mode}

During Mode-II loading, the displacement of the crack surface is in the plane of the crack and perpendicular to the leading edge of the crack. This is caused by in-plane shear. Mode-II crack tip stress and displacement fields are given by Irwin [32]: 


$$
\begin{gathered}
{\left[\begin{array}{c}
\sigma_{r} \\
\sigma_{\theta} \\
\tau_{r \theta}
\end{array}\right]=\frac{K_{I I}}{\sqrt{2 \pi r}}\left[\begin{array}{c}
\sin \frac{\theta}{2}\left(1-3 \sin ^{2} \frac{\theta}{2}\right) \\
-3 \sin \frac{\theta}{2} \cos ^{2} \frac{3 \theta}{2} \\
\cos \frac{\theta}{2}\left(1-3 \sin ^{2} \frac{\theta}{2}\right)
\end{array}\right]} \\
\sigma_{z}=v\left(\sigma_{r}+\sigma_{\theta}\right) \\
\sigma_{z}=\tau_{r z}=\tau_{\theta z} \\
\tau_{r z}=\tau_{r \theta}=0 \\
{\left[\begin{array}{c}
u \\
v \\
w
\end{array}\right]=\frac{K_{I I}}{4 G} \sqrt{\frac{r}{2 \pi}}\left[\begin{array}{c}
2(\kappa-1) \sin \frac{\theta}{2}+3 \sin \frac{3 \theta}{2} \\
-2(\kappa-1) \cos \frac{\theta}{2} \\
0
\end{array}\right]}
\end{gathered}
$$

In Mode-II the material failure occurs when $K_{\mathrm{II}}$ reaches its critical value $K_{\mathrm{IIc}}$, known as the Mode-II fracture toughness.

\subsubsection{Mode-III or tearing mode}

In this mode, the crack surface displacements are in the plane of the crack and parallel to its leading edge. This is caused by out-of-plane shear. Mode-III near tip stress and displacement fields are given as Irwin [32]:

$$
\begin{gathered}
{\left[\begin{array}{c}
\tau_{r z} \\
\tau_{\theta_{z}}
\end{array}\right]=\frac{K_{I I I}}{\sqrt{2 \pi r}}\left[\begin{array}{r}
\sin \frac{\theta}{2} \\
\cos \frac{\theta}{2}
\end{array}\right]} \\
\sigma_{z}=\sigma_{r}=\sigma_{\theta}=\tau_{r \theta}=0 \\
w=\frac{1}{G} \int \sigma_{x z} d x=\frac{2 K_{I I I}}{G} \sqrt{\frac{r}{2 \pi}} \sin \frac{\theta}{2} \\
u=v=0
\end{gathered}
$$

The elastic stress and displacement fields in mixed mode problems may be obtained from the above results using the superposition principle. For Mode-I and Mode-II combination, for example, this yields:

$$
\left[\begin{array}{c}
\sigma_{r} \\
\sigma_{\theta} \\
\tau_{r \theta}
\end{array}\right]=\frac{K_{I}}{\sqrt{2 \pi r}}\left[\begin{array}{c}
\cos \frac{\theta}{2}\left(1+\sin ^{2} \frac{\theta}{2}\right) \\
\cos ^{3} \frac{\theta}{2} \\
\sin \frac{\theta}{2} \cos ^{2} \frac{\theta}{2}
\end{array}\right]+\frac{K_{I I}}{\sqrt{2 \pi r}}\left[\begin{array}{c}
\sin \frac{\theta}{2}\left(1-3 \sin ^{2} \frac{\theta}{2}\right) \\
-3 \sin \frac{\theta}{2} \cos ^{2} \frac{\theta}{2} \\
\cos \frac{\theta}{2}\left(1-3 \sin ^{2} \frac{\theta}{2}\right)
\end{array}\right]
$$


In the preceding expressions, $G$ represents the shear modulus. The above stresses and displacements can be expressed in the integral form as:

$$
\begin{gathered}
\sigma_{i j}=\frac{K_{I}}{\sqrt{2 \pi r}} f_{I_{i j}}(\theta)+\frac{K_{I I}}{\sqrt{2 \pi r}} f_{I I_{i j}}(\theta) \\
u_{i}=\frac{K_{I}}{4 g} \sqrt{\frac{r}{2 \pi}} g_{I_{i}}(\theta)+\frac{K_{I I}}{4 g} \sqrt{\frac{r}{2 \pi}} g_{I I_{i}}(\theta)
\end{gathered}
$$

where the stress intensity factors are defined as:

$$
\begin{gathered}
K_{I}=\sigma_{n} \sqrt{\pi a}=\sigma \sqrt{\pi a} \sin ^{2} \beta \\
K_{I}=\sigma_{n} \sqrt{\pi a}=\sigma \sqrt{\pi a} \sin \beta \cos \beta
\end{gathered}
$$

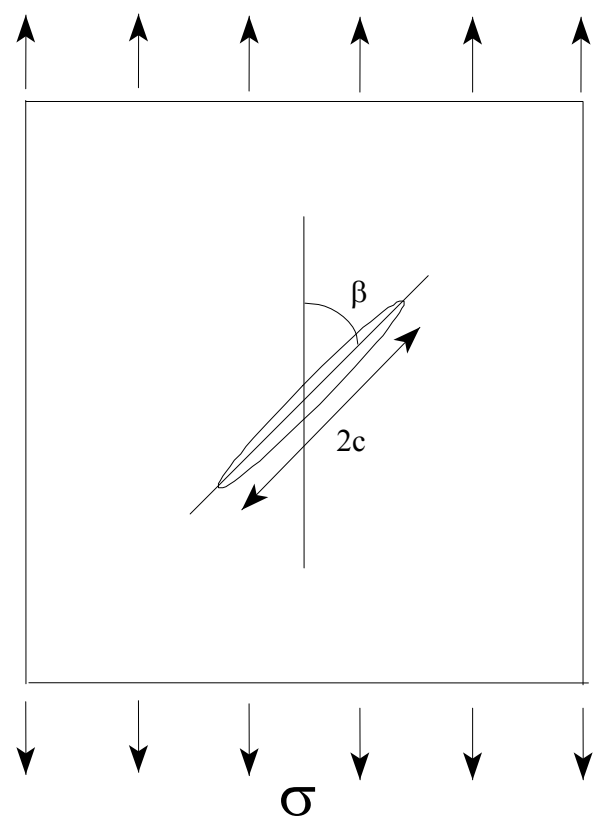

Figure 2.3. Geometry of an inclined crack.

The above equations show that $K_{\mathrm{I}}$ and $K_{\mathrm{II}}$ are function of the crack inclination angle, $\beta$ (see Figure 2.3). Thus, in mixed-mode, the stress intensity factors are functions of the applied load, crack dimensions, and crack inclination with respect to vertical.

These equations form the mathematical framework for fracture propagation studies using the Linear Elastic Fracture Mechanics (LEFM). It can be seen that displacements are proportional to $\sqrt{ } \mathrm{r}$ and that whatever the mode of loading, the stresses contain a singularity of the form $\left(1 /\left(V_{r}\right)\right)$. Therefore, the stress field becomes infinite at the tip for all values of $\mathrm{K}$ not equal to zero, for any arbitrary loading. It is apparent that infinite stresses are physically unacceptable and are a direct result of the simplified model used to describe the real physical state. The model assumes perfectly sharp cracks and linear 
brittle material behavior. But the development of plastic zones ahead of cracks in metals, and micro-cracking in rocks are manifestations of non-linear behavior and reduce the tip stresses to a finite value. This region of micro-cracking ahead of a fracture in rocks is often referred to as the process zone and differs from the region of yielding near the crack tip in metals. For rocks, the size and shape of this zone remain the same whether the crack tip location is a free surface or deep within the material, i.e., for plane stress and plane strain. This does not hold for metals [35]. It is for this reason that laboratory observations indicate that thickness has no effect on fracture toughness of rocks. So long as the size of the process zone remains small relative to other dimensions of the problem, linear elastic conditions prevail, and LEFM remains applicable.

To resolve the problem of stress singularity at the tip, Barenblatt [36] proposed a new theory in which two postulates were invoked when characterizing the crack tip: (1) that there are intense cohesive forces that act over a small region at the ends of the crack, and (2) that the local distribution of these cohesive forces is the same for a given material under specified conditions. The stress singularity arising from these cohesive forces is such that it cancels out the stress singularity resulting from the applied stresses. This, according to Goodier [37], can be regarded as a third postulate of Barenblatt's theory of equilibrium cracks. The consequence of the third postulate is that the crack closes smoothly and the crack opening displacement has a slope of zero at the tip (Figure 2.4).

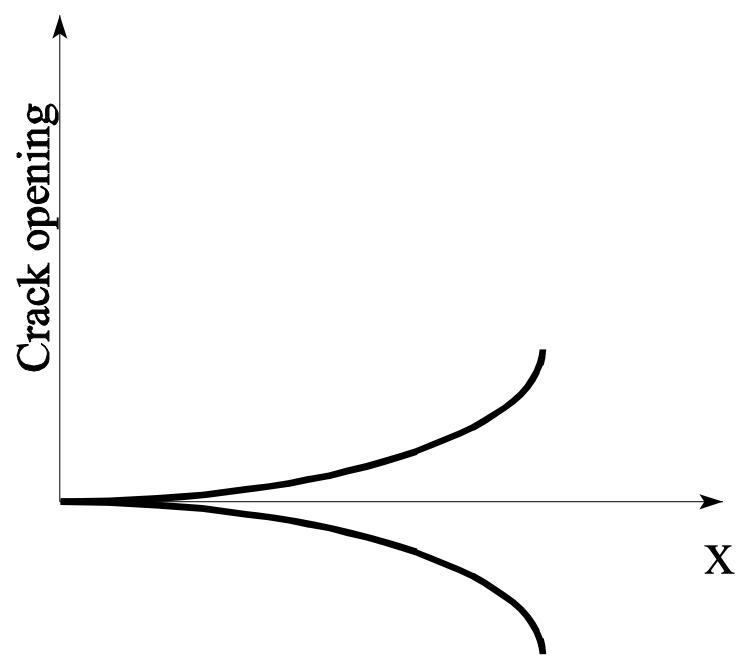

Figure 2.4. Crack-tip closure resulting from Barenblatt's $3^{\text {rd }}$ postulate.

This is in contrast to the opening of a Griffith crack used in this work which is elliptical with a finite radius of curvature and closes with an infinite slope at the tip. The theory of Barenblatt [36] suggests that the cohesive forces increase in response to an increase, from zero, of the applied load on the crack. When these forces are unable to keep the crack from opening, the crack becomes a mobile equilibrium crack and propagation ensues. Barenblatt [36] introduced a new material property called the modulus of cohesion defined as: 


$$
K_{0}=\int_{0}^{d} \frac{g(\xi)}{\xi^{\frac{1}{2}}} d \xi
$$

in which $\mathrm{g}(\xi)$ is a function describing the intensity of the cohesive forces over a small interval, $\mathrm{d}$, at the tip of the crack. The form of this function is unknown so that the above integral cannot be performed. However, Barenblatt showed that it can be expressed in terms of elastic modulus and the surface energy, $\gamma$, as:

$$
K_{0}=(\pi \gamma E)^{\frac{1}{2}}
$$

Using the concept of the stress intensity factor, $K$, Barenblatt's fracture criterion can be written as:

$$
K=\frac{K_{0}}{\pi}
$$

which can be compared to the Griffith's stress condition by using $K=\sigma \sqrt{ }(2 \pi a)$ to obtain the stress required for fracture:

$$
\sigma_{t}=\sqrt{\frac{1}{2 \pi}} \sqrt{\frac{2 E \gamma}{\pi a}}
$$

This differs from the Griffith criterion, Eq. (2.16), by only a constant.

The Griffith-Irwin criterion is a useful one when applied to situations where the fracture propagates in its own plane. However, the direction of crack propagation is not known a priori under combined loading situations so that the above criterion is inadequate and other propagation theories are needed. There are a number of such theories that can be divided into two groups, namely local criteria and global criteria. In both groups, propagation is assumed to occur at a point where the value of the parameter of interest reaches a critical value characteristic of the material. The propagation direction is determined by the direction in which this parameter has its minimum or maximum as required by the criterion.

\subsection{Fracture Propagation Criteria for Combined Loading}

2.3.1. Local propagation criteria: The maximum circumferential tensile stress, $\sigma_{\theta \max }$, criterion [38] and the minimum strain energy density, $S_{\theta \min }$, [39] are examples of local propagation criteria. Propagation is determined by the value of these parameters at a distance from the crack tip. In the $\sigma_{\theta \max }$-theory, proposed by Erdogan and Sih [38], the crack extension starts at the tip, in a radial direction, and in a plane perpendicular to the direction of maximum tension. Fracture propagates when $\sigma_{\theta \max }$ reaches its critical value. 
The $\mathrm{S}_{\theta \min }$-theory was formulated by Sih [39]. In this theory, the parameter governing fracture propagation is the strain density at the point of propagation. The initial crack growth occurs in the direction along which the strain energy density factor possesses a stationary (minimum) value. The crack extends when the strain energy density reaches a critical value, $\mathrm{S}_{\theta}$, a material property. The value of $\mathrm{S}_{\mathrm{c}}$ is evaluated along a contour $r=r_{0}$, with $r_{0}$ being a property of the material.

2.3.2. Global propagation criteria: are based on the maximum energy release rate, $G_{(\theta) \max }$, associated with crack propagation [40-42). The global energy approach has its roots in the Griffith criterion and is based on the universal principle of energy minimization. It predicts that a fracture will grow in the direction along which the elastic energy release per unit crack extension will be maximum and the crack will start to grow when this energy release rate reaches a critical value. However, according to Sih and Erdogan [43], the application of the energy release rate concept to cracks under mixed mode loading are of doubtful value due to the mathematical difficulties caused by prevailing stress singularities at the crack tip and corner where it is kinked, and because of the problems with accuracy of numerical solutions for solving problems involving stress singularities. Despite the above arguments and that there is no clear reason why the propagation path has to follow the direction of maximum $G_{\theta}$, and authors in $[40,41,44,45]$ use the above approach to arrive at propagation theories for mixed mode problems involving metals.

From a numerical modeling point of view, the computational effort required to predict the propagation path is very important. The energy release rate needs to be calculated numerically by taking the derivative of the energy release, $G$, with respect to the fracture length or area in the direction of propagation. The direction of propagation is not known a priori so that a trial-and-error method is needed for its determination. For threedimensional problems, this procedure has to be repeated at several locations along the fracture edge (at every node when there is a possibility of branching). This makes the energy release rate criterion relatively computationally intensive.

\subsubsection{Structural (non-local) criteria}

The above review indicates that since Griffith's paper [7] on fracture of brittle materials in compression, immense literature has appeared on the subject (see also reviews and references in [47-59]). Most of these works has focused on the opening mode or mode I (tensile) propagation. Meanwhile, in many cases of significance in rock mechanics, the mode II (sliding, shear) propagation may prevail; or it may appear at some stage of crack growth. These situations arise in the presence of sufficiently high confining pressure [51,56-58], or even under uniaxial compression [51,59-60] and many cases of crack coalescence [51-52,55,57,59-60]. 
The importance of mode II crack propagation in rock is evident by the considerable attention it has received both analytically [51,56,58,61-62] and numerically [53,57-60,6364]. These investigations have provided fundamental results and understanding, however, it is necessary to develop a unified crack initiation criterion that is capable of predicting both tensile and shear cracks [63], and to establish a reliable fracture criterion for predicting Mode II fracture [56]. Such a criterion has been developed [64,65] and is used in this work. Below, we describe certain features of this criterion.

\subsection{A Unified criterion for Crack Propagation and Coalescence under Compressive Loads}

'Structural' criteria which are often called 'non-local' use a structural parameter, $d$. One such approach employs the criterion of average tensile stress over a characteristic size, $d$, of a fracture process zone (FPZ):

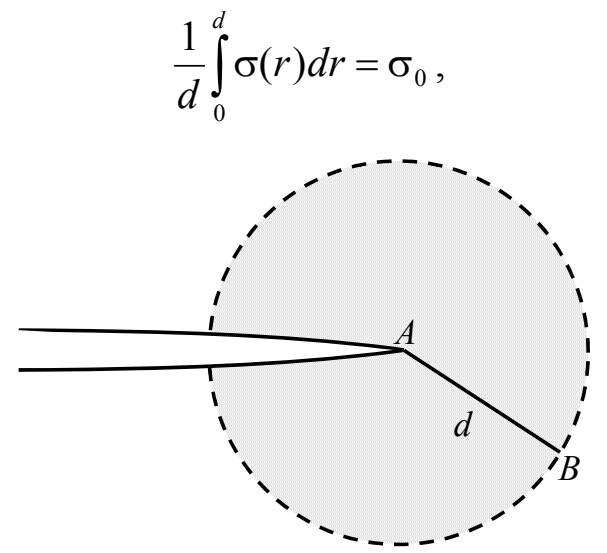

Fig. 2.5. Graphical interpretation of the edge and structural criteria. Edge criterion: the stress is calculated at a point $B$ at a distance equal to the length $d$ of the FPZ; Structural criterion: average stress over the segment $\mathrm{AB},|A B|=d$.

where $\sigma_{0}$ has the meaning of the microscopic tensile strength; here and henceforth, tensile stresses are assumed positive. An alternative formulation of 2.22 employs the tensile stress at the edge of the FPZ at a distance $d$ rather than the average value, i.e.:

$$
\sigma(d)=\sigma_{0}
$$

For a small FPZ, when $d / \ell \rightarrow 0$, the criteria (2.22), (2.23) are equivalent to the propagation condition in terms of the SIFs:

$$
K_{\mathrm{I}}=K_{\mathrm{IC}}
$$

with $K_{\mathrm{IC}}=\sigma_{0} \sqrt{\pi d / 2}$ for (2.22); and $K_{\mathrm{IC}}=\sigma_{0} \sqrt{2 \pi d}$ for (2.23). In this case, the asymptotic formulae provide easy extensions and simple analytical results.

From a computational viewpoint, average values are less sensitive to local errors and thus provide a better opportunity for comparing the results obtained by various methods. Finally, average values agree with the concept of the finite element method which 
employs stresses averaged over an element, and which is often employed for simulation of crack propagation e. g., [56,58-59] and [68, 85-87].

Criterion (2.22) in its initial form referred to tensile fractures, but its concept is quite general and can obviously be extended to other types of fracture [74-77]. It is sufficient to formulate the macroscopic condition of fracture (tensile, shear, time-dependent, etc.) in terms of stresses $f\left(\sigma_{i j}\right)=\sigma_{0}$ and substitute the values averaged over a characteristic size $d$ into it. Thus, one obtains the criterion:

$$
f\left(\overline{\sigma_{i j}}\right)=\sigma_{0}
$$

where:

$$
\overline{\sigma_{i j}}=\frac{1}{d} \int_{0}^{d} \sigma_{i j}(r) d r
$$

Note that (2.21) may be considered as an extension of (2.23) as well, if it is assumed that:

$$
\overline{\sigma_{i j}}=\sigma_{i j}(d)
$$

The function $f\left(\sigma_{i j}\right)$ and the strength $\sigma_{0}$ are different for tension and compression. The structural parameter $d$ may also differ. We will call the function $f\left(\overline{\sigma_{i j}}\right)$ and the parameter $\sigma_{0}$ as a driving force and the microscopic strength, respectively; and refer to the ratio $F=f\left(\overline{\sigma_{i j}}\right) / \sigma_{0}$ as a normalized driving force. A criterion of the form (2.25) and (2.26), including the average normal $\overline{\sigma_{n n}}$ and shear $\overline{\sigma_{n t}}$ tractions as a particular choice of $\overline{\sigma_{i j}}$, was suggested and successfully employed in [72] in 2D. The most general form of (2.25) was suggested in [74], where the driving force $f$ was taken as an arbitrary functional.

It is of special significance to appropriately prescribe the parameters $d$ and $\sigma_{0}$ that enter (2.25), (2.26). In practice, they are to be found experimentally using specimens having thin slits. Since direct measurements of $\sigma_{0}$ are not feasible, the following consideration is useful. The form (2.25), (2.26) does not include the length $2 l$ of a crack: the latter may be either much greater than the structural parameter $d$, or it may exceed the latter not too significantly (four-ten times). In the case when the relative length of a crack is large ( $d / l$ $<<1)$, the asymptotic form of (2.25), (2.26), formulated in terms of the SIFs and discussed in Sec. 4, becomes available. The asymptotic form does not contain the values of $d$ and $\sigma_{0}$ separately: they enter as the combination $\sqrt{d} \sigma_{0}$ defining a critical SIF, $K_{C}=\sqrt{(\pi / 2) d} \sigma_{0}$. Hence, instead of measuring $\sigma_{0}$, one may estimate the critical SIF in an experiment with a sufficiently long crack (from our calculations it follows that it is sufficient to have $d / l<0.1)$. Then, for a prescribed $d$ we find the value $\sigma_{0}=K_{C} \sqrt{2 /(\pi d)}$. Now the values of $d$ and $\sigma_{0}$ may be used for shorter cracks, when the approximation of the small FPZ is not valid. Thus, the critical SIF for a small FPZ may serve to estimate $\sigma_{0}$ in the general case. 
A number of particular equations for the function $f$ have been listed in $[72,74]$ that deal with the case of tensile and bending external loads. But in the rock mechanics where the applied loads are primarily compressive other particular equations for the driving force are considered. For mode I, we will use the common choice:

$$
f_{\mathrm{I}}\left(\overline{\sigma_{i j}}\right)=\overline{\sigma_{\theta \theta}}, \sigma_{0}=\sigma_{t}
$$

where $\overline{\sigma_{\theta \theta}}>0, \sigma_{t}$ is the tensile strength. For mode II, the Mohr-Coulomb criterion seems appropriate:

$$
f_{\text {II }}\left(\overline{\sigma_{i j}}\right)=\operatorname{sign}\left(\overline{\sigma_{r \theta}}\right) \overline{\sigma_{r \theta}}-\tan \rho \cdot\left(-\overline{\sigma_{\theta \theta}}\right), \sigma_{0}=c
$$

where $\overline{\sigma_{\theta \theta}} \leq 0, \rho$ is the angle of internal friction, $c$ is cohesion.

Each of these equations has been suggested in [25] where a unified criterion, the combination of another function $f_{\mathrm{I}}=\sqrt{{\overline{\sigma_{\theta \theta}}}^{2}+\left(\overline{\sigma_{r \theta}} \sigma_{t} / c\right)^{2}}$ for tension and the particular form $f_{\mathrm{II}}=\operatorname{sign}\left(\overline{\sigma_{r \theta}}\right) \overline{\sigma_{r \theta}}$ for compression, was used. This combination has been studied in detail for a small FPZ near a notch with the angle equal to $0^{\circ}$ (a crack), $20^{\circ}$, and $40^{\circ}$. The unified criterion presented and used herein to numerically simulate crack propagation under compressive loads is given by:

$$
f\left(\overline{\sigma_{i j}}\right)=\left\{\begin{array}{cc}
f_{\mathrm{I}}\left(\overline{\sigma_{i j}}\right)=\overline{\sigma_{\theta \theta}}=\sigma_{\mathrm{t}} & \overline{\sigma_{\theta \theta}}>0 \\
f_{\mathrm{II}}\left(\overline{\sigma_{i j}}\right)=\operatorname{sign}\left(\overline{\sigma_{r \theta}}\right) \sigma_{r \theta}-\tan \rho \cdot\left(-\overline{\sigma_{\theta \theta}}\right)=c & \overline{\sigma_{\theta \theta}} \leq 0
\end{array}\right.
$$

Including the friction angle $\rho$ into the second of (2.28) provides an additional parameter for better approximation. It tends to account for the influence of the normal compressive traction on the shear bearing capacity of a material at the scale of a FPZ. We believe that this influence may be significant, as is the case for intact rock and surfaces in contact. The parameter $\rho$ is to be found in experiments, for instance, of the type suggested in [56]. Presently, not having such experimental data, we may only speculate whether the values of $\rho$ in (2.28) will agree with those obtained in compression of specimens and/or with those obtained for surfaces in contact. Expression (2.28) was used in [8] with $\overline{\sigma_{i j}}$ defined by (2.27) and with $\rho=0$. Note that in the case of a small FPZ with $\rho=0$, an asymptotic form of (2.28) written in terms of SIFs was used in [58,56]. In 3D, $\theta$ is the angle between the crack plane and a plane of possible propagation; $\operatorname{sign}\left(\overline{\sigma_{r \theta}}\right) \sigma_{r \theta}$ is taken as the absolute value of the shear traction in the propagation plane: $\operatorname{sign}\left(\overline{\left.\sigma_{r \theta}\right) \sigma_{r \theta}}=\sqrt{{\overline{\sigma_{\xi \theta}}}^{2}+{\overline{\sigma_{\eta \theta}}}^{2}}\right.$, where $\overline{\sigma_{\xi \theta}}$ and $\overline{\sigma_{\eta \theta}}$ are components of the shear traction in the local co-ordinates $\xi, \eta$ in this plane with $\xi$ normal to a crack front. Thus, the classical mode II and III are included as particular cases when either $\overline{\sigma_{\eta \theta}}$, or $\overline{\sigma_{\xi \theta}}$ equals zero.

\subsubsection{Mode and direction of crack propagation}

For a crack front moving in a fixed mode, the direction of propagation is that for which the driving force $f$ and, consequently, the normalized driving force $F$ is a maximum. In particular, if the direction is characterized by the angle $\theta$ (Fig. 2.5), the angle of propagation is: 
for mode I:

$$
\theta_{\mathrm{I}}=\left\{\theta: \max _{\theta} F_{\mathrm{I}}(\theta)\right\}
$$

for mode II:

$$
\theta_{\mathrm{II}}=\left\{\theta: \max _{\theta} F_{\mathrm{II}}(\theta)\right\}
$$

The corresponding maximum normalized driving forces are $F_{\mathrm{Imax}}=F_{\mathrm{I}}\left(\theta_{\mathrm{I}}\right)$ and $F_{\mathrm{IImax}}=$ $F_{\text {II }}\left(\theta_{\text {II }}\right)$, respectively. At a position of the crack tip where both evaluated $F_{\text {Imax }}$ and $F_{\text {IImax }}$ are positive, the condition of propagation $f\left(\overline{\sigma_{i j}}\right) \geq \sigma_{0}$ is satisfied for both angles $\theta_{\mathrm{I}}$ and $\theta_{\text {II }}$, so that it is necessary to choose between the modes of propagation. This is achieved by assuming that at such a point, the crack will move in the direction corresponding to the greater of the maximum normalized driving forces $F_{\text {Imax }}$ and $F_{\text {IImax }}$. Thus,

mode I occurs if $F_{\text {Imax }}>0$ and:

mode II occurs if $F_{\text {IImax }}>0$ and:

$$
F_{\text {Imax }}>F_{\text {IImax }}
$$

$$
F_{\text {IImax }}>F_{\text {Imax }}
$$

With $\overline{\sigma_{i j}}$ defined by (2.27) and with $\rho=0$, the conditions (2.31), (2.32) were used in [8] to determine the mode of propagation. In the case of a small FPZ with $\rho=0$, the asymptotic form of (2.32) written in terms of SIFs turns into (2.21). As mentioned, the latter was used in $[58,56]$.

The direction $\theta_{\mathrm{p}}$ of fracture propagation is thus defined by:

$$
\theta_{\mathrm{P}}=\left\{\theta_{\mathrm{P}}: \max _{\theta_{\mathrm{I}}, \theta_{\mathrm{II}}}\left[F_{\text {Imax }}=F_{\mathrm{I}}\left(\theta_{\mathrm{I}}\right), F_{\text {IImax }}=F_{\text {II }}\left(\theta_{\text {II }}\right)\right]\right\}
$$

Because usually the angles $\theta_{\mathrm{I}}$ and $\theta_{\mathrm{II}}$ differ, the point corresponding to the change in the propagation mode is a kink point. Equations (2.28), (2.29)-(2.33) are applicable at every point along a crack trajectory, i.e., for initial cracks, for smooth portions of a trajectory, at kink points, for secondary cracks, and for coalescence. In general, a numerical implementation of (2.29), (2.30) necessitates a search among the angles using procedures that involve direct calculations of $f_{\mathrm{I}}\left(\overline{\sigma_{i j}}\right)$ and $f_{\mathrm{II}}\left(\overline{\sigma_{i j}}\right)$. In the case of a small FPZ, the search may employ analytical formulae.

In summary, to find the direction of crack propagation, normalized normal and shear driving forces are evaluated over a fracture process zone (FPZ). Directions comprising angles from $-\pi$ to $+\pi$ with the current tip elements are checked. Driving forces are evaluated ahead of the crack tip in the direction to be checked over the distance equal to the length of a fracture process zone (FPZ). The direction for which the normalized tensile or shear driving force is maximum is determined and a new element is added in this direction. 


\section{References}

1. Erdogan, F. 1974. Principles of fracture mechanics. In Thoft-Christensen (ed.) Continuum Mechanics Aspects of Geodynamics and Rock Mechanics, D. Reidel publishing, Dordrecht-Holland, 29-44.

2. Jaeger, J.C. \& Cook, N.G.W., 1979. Fundamentals of rock mechanics. 3rd ed., Chapman and Hall, New York.

3. Obert, L., 1972. Brittle fracture of rock. In H. Liebowitz (ed.) Fracture: An Advanced Treatise, VII, 93-155.

4. Brace, W.F., 1960. An extension of the Griffith's theory of fracture to rocks. J. Geophys. Res., 65(10), 3477-3480.

5. Griffith, A.A., 1921. The theory of rupture and flow in solids. Phil. Trans. Roy. Soc. London, 163-197.

6. Inglis, C.E., 1913. Stresses in a plate due to the presence of cracks and sharp corners. Trans. Inst. Naval Architects, 55, 219-230.

7. Griffith, A.A., 1924. Theory of rupture. Proc. of the 1st Int. Cong. Appl. Mech., Delft, 55-63.

8. Rinehart, J.S., 1966. Fracture of rocks. Int. J. Fract. Mech., 2(3), 534-551.

9. Murrell, S.A.F., 1958. The strength of Coal under triaxial compression. In W.H. Walton (ed.) Mechanical Properties of Nonmetallic Solids, Interscience, New York, 123-146.

10. Brace, W.F., 1964. Brittle fracture of rocks, In W.R. Judd (ed.) State of Stress in the Earth's Crust. Elsevier, New York, 111-174.

11. McClintock, F.A. \& Walsh, J.B., 1962. Friction on Griffith cracks under pressure. Proc. of the 4th U.S. National Cong. of Appl. Mech., 1015-1577.

12. Goodman, R.E., 1980. Introduction to rock mechanics. John Wiley \& Sons, New York.

13. Daneshy, A.A., 1973. Experimental investigation of hydraulic fracturing through perforations. J. Pet. Tech., Oct., 1201-1206.

14. Ong, S.H., 1994. Borehole Stability. Ph.D. dissertation, Dept. of Petroleum and Geological Engineering, The University of Oklahoma.

15. Solberg, P., Lockner, D. \& Byerlee, J., 1977. Shear and tension hydraulic fractures in low permeability rocks. Pure Appl. Geophys., 115, 191-198.

16. Callanan, M.J., 1981. Hydraulic fracture initiation by shear failure in formations at great depths. Proc. Workshop on hydraulic fracturing stress measurements, Monterey, CA, 181-189. 
17. Paulding, B.W., 1967. Orientation of hydraulically induced fractures. Proc. 9th Symp. on Rock Mech., Golden, Colorado, 461-489.

18. Guo, F., Morgenstern, N.R. \& Scott, J.D., 1993. Interpretation of hydraulic fracturing breakdown pressure. Int. J. Rock Mech. Min. Sci. \& Geomech. Abstr., 30(6), 617-626.

19. Rummel, F., 1987. Fracture mechanics approach to hydraulic fracturing stress measurements. In B.K. Atkinson (ed.) Fracture Mechanics of Rocks, 217-240.

20. Zoback M.D., Rummel F., Jung R. \& Raleigh C.B., 1977. Laboratory hydraulic fracturing experiments in intact and pre-fractured rock. Int. J. Rock Mech. Min. Sci. Geomech. Abstr, 14, 49-58.

21. Abou-Sayed, A.S., Brechtel C.E. \& Clifton R. J., 1978. In situ stress determination by hydrofracturing: a fracture mechanics approach. J. Geophys. Res., 83(B6), 2851-2862.

22. Rummel, F. \& Hansen J., 1989. Interpretation of hydrofrac pressure recordings using a simple fracture mechanics simulation model. Int. J. Rock Mech. Min. Sci. Geomech. Abstr, 26, 483-488.

23. Ito, T. \& Hayashi, K., 1991. Physical background to the breakdown pressure in hydraulic fracturing tectonic stress measurements. Int. J. Rock Mech. Min. Sci. Geomech. Abstr, 28, 285-293.

24. Hubbert M.K. \& Willis D.G., 1957. Mechanics of hydraulic fracturing. Trans. Am. Inst. Min. Engrs., 210, 153-168.

25. Severin A., Mroz Z. A non-local stress failure condition for structural elements under multiaxial loading. Eng. Fracture Mech., 1995, 51, 955-973.

26. Stephens, G. \& Voight, B., 1982. Hydraulic fracturing theory for conditions of thermal stress. Int. J. Rock Mech. Min. Sci. \& Geomech. Abst., 19, 279-284.

27. Nygren A., and A. Ghassemi, 2004. An estimate of $S_{H \max }$ from drilling-induced tensile fractures in Coso geothermal reservoir, CA. Poster Presentation 2004 GRC annual meeting, Palm Springs, CA.

28. Ghassemi, A. \& Zhang, Q. 2004. A transient fictitious stress boundary element method for poro-thermoelastic media. J. Eng. Anal. Boundary Elements, 28/11, 1363-1373.

29. Kanninen, M.F. \& Popelar, C.H., 1985. Advanced fracture mechanics. Oxford University Press, New York.

30. Westergaard, H.M., 1939. Bearing pressures and cracks. J. Appl. Mech., 6(2), 49-53.

31. Sneddon, I.N., 1946. The distribution of stress in the neighborhood of a crack in an elastic solid. Proc. Royal Soc. London, Ser. A., 187, 229-260.

32. Irwin, G.R., 1957. Analyses of stresses and strains near the end of a crack transversing a plate. J. Appl. Mech., 24, 361-364. 
33. Roegiers, J.-C. \& Zhao, X.L., 1991. Rock fracture tests in simulated downhole conditions. Proc. of the 32nd U.S. Symposium on Rock Mechanics, Rock mechanics as a Multidisciplinary Science, A.A. Balkema, 221-230.

34. Boone, T.J., Ingraffea, A.R. \& Roegiers, J.-C., 1991. Simulation of hydraulic fracture propagation in poroelastic rock with application to stress measurement technique. Int. J. Numer. Anal. Meth. Geomech., 28, 1-14.

35. Schmidt, R.A., 1980. A microcrack model and its significance to hydraulic fracturing and fracture toughness testing. Proc. 21st Rock Mech. Symp., 581-590.

36. Barenblatt, G.I., 1962. The mathematical theory of equilibrium cracks. In: Brittle Fracture, Advances in Appl. Mech., 7, 55-129.

37. Goodier, J.N. 1972. Mathematical theory of equilibrium cracks. In H. Liebowitz (ed.) Fracture: An Advanced Treatise, 2, 1-66.

38. Erdogan, F. \& Sih, G.C., 1963. On the crack extension in plates under plane loading and transverse shear. Trans. ASME, J. Basic Engrg., 519-527.

39. Sih, G.C., 1974. Strain-energy-density factor applied to mixed mode crack problems. Int. J. fracture, 10(3), 305-320.

40. Hussain, M.A., Pu, S.,L. \& Underwood, J., 1974. Strain energy release rate for a crack under combined Mode I and Mode II. In Fracture Analysis, Proc. of the '73 Nat. Symp. on fracture mechanics, part II, ASTM STP 560, ASTM, 2-28.

41. Shah, R.C., 1974. Fracture under combined modes in 4340 Steel. In: Fracture Analysis. Proc. of the 1973 national symposium on fracture mechanics, part II, ASTM STP 560, ASTM, 29-52.

42. Tirosh, J., 1977. Incipient fracture angle, fracture loci and critical stress for mixed mode loading. Engrg. Fract. Mech., 9, 607-616.

43. Sih, G.C. \& Erdogan, F., 1963. Stress intensity factor: concept and application. Linear Fracture Mechanics, Sih G. C., Wei, R.P., \& Erdogan F. (eds.), Envo Publishing Company, 85-106.

44. Palaniswami, K. \& Knauss, W.G., 1978. On the problem of crack extension in brittle solids under general loading. In Nemat-nasser (ed.) Mechanics Today, Vol. 4, Pergamon Press, New York, 87-148.

45. Yatomi, C., Fujii, K. \& Nakagawa, K., 1989. Combined stress hypothesis for mixed mode fracture toughness criterion. Engrg. Fracture Mech., 32(6), 881-888.

46. Nemat-Nasser S., Horii H. Compression-induced nonplanar crack extension with application to splitting, exfoliation and rockburst. J. Geophysical Research, 1982, 87, 6805-6821.

47. Horii H., Nemat-Nasser S. Compression-induced microcrack growth in brittle solids: axial splitting and shear failure. J. Geophysical Research, 1985, 90, 3105-3125. 
48. Kemeny J. M., Cook N. G. W. Micromechanics of deformation in rocks. Toughness Mechanics in Quasi-Brittle Materials. S. P. Shah (ed.). Kluwer Academic Publishers, the Netherlands, 1991, 155-188.

49. Kachanov M. L. Elastic solids with many cracks and related problems. Advances in Applied Mechanics, 1993, 30, 259-445.

50. Bobet A., Einstein H.H. Fracture coalescence in rock-type materials under uniaxial and biaxial compression. Int. J. Rock Mech. Min. Sci., 1998, 35, 863-888.

51. Wong R.H.C., Chau K.T. Crack coalescence in rock-like material containing two cracks. Int. J. Rock Mech. Min. Sci., 1998, 35, 147-164.

52. Bobet A., Einstein H.H. Numerical modeling of fracture coalescence in a model rock material. Int. J. of Fracture, 1998, 92, 221-252.

53. Dyskin A. V. Stress fluctuation mechanism of mesocrack growth, dilatancy and failure of heterogeneous materials in uniaxial compression. HERON, 1998, 43, 137-158.

54. Wong R.H.C., Chau K.T., Tang C.A., Lin P. Analysis of crack coalescence in rock-like materials containing three flaws - Part I: experimental approach. Int. J. Rock Mech. Min. Sci., 2001, 38, 909-924.

55. Rao Q., Sun Z., Stephansson O, Li C., Stillborg B. Shear fracture (Mode II) of brittle rock. Rock Mechanics and Mining Sciences, 2003, 40, 355-375.

56. De Bremaecker J.-C., Ferris M.C. Numerical models of shear fracture propagation. Eng. Fracture Mech., 2004 (in press).

57. Melin S. When does a crack grow under mode II conditions? Int. J. Fracture. 1986, 30, 103-114.

58. Reyes O., Einstein H. H. Failure mechanism of fractured rock - a fracture coalescence model. Proc. 7th Int. Congress of Rock Mechanics, 1991, 1, 333-340.

59. Shen B., Stephanson O., Einstein H. H., Ghahreman B. Coalescence of fractures under shear stress in experiments. J. Geophysical Research, 1995, 100, 5975-5990.

60. Cherepanov G.P. Propagation of cracks in compressed bodies. J. Applied Math. Mech. (English translation of Prikladnaia Matematika i Mekhanika), 1966, 30, 96-109.

61. Isaksson P., Stale P. Prediction of shear crack growth direction under compressive loading and plane strain conditions. Int. J. Fracture, 2002, 113, 175-194.

62. Bobet A. Numerical simulation of initiation of tensile and shear cracks. Rock Mechanics in the National Interest. Proc. of the $38^{\text {th }}$ US Rock Mechanics Symposium. Tinucci \& Heasley (eds). Swets \& Zeitlinger Lisse, 2001, 731-738.

63. Tang C.A., Lin P., Wong R.H.C., Chau K.T. Analysis of crack coalescence in rock-like materials containing three flaws - Part II: numerical approach. Int. J. Rock Mech. Min. Sci., 2001, 38, 925-939. 
64. Dobroskok, A. A., Ghassemi, A., and Linkov, A.M. 2005. Novozhilov structural criterion: an extension for numerical simulation of mode I and II crack growth and coalescence. Int. J. of Fracture. 133, 223-246.

65. Dobroskok, A. A., Ghassemi, A., and Linkov, A.M. 2005. Numerical simulation of crack propagation influenced by thermal and porous liquid stresses. Int. J. of Fracture. 134, L29-L34.

66. Petit J.-P., Barquins M. Can natural faults propagate under mode II conditions? Tectonics, 1988, 7, 1243-1256.

67. Dyskin A. V. Crack growth criteria incorporating non-singular stresses: size effect in apparent fracture toughness. Int. J. Fracture, 1997, 83, 191-206.

68. Neuber H. Theory of Notch Stresses. J.W. Edwards, Ann Arbor, Michigan, 1946.

69. Novozhilov V.V. On a necessary and sufficient criterion for brittle strength. J. Applied Math. Mech. (English translation of Prikladnaia Matematika i Mekhanika), 1969, 33, 201-210.

70. Severin A. Brittle fracture criterion for structure with sharp notches. Eng. Fracture Mech., 1994, 47, 673-681.

71. Mikhailov S. E. A functional approach to non-local strength conditions and fracture criteria. - I. Body and point fracture. II. Discrete fracture. Eng. Fracture Mech., 1995, 52, 731-743, 745-754.

72. Isupov I. P., Mikhailov S. E. A comparative analysis of several nonlocal fracture criteria. Archive for Applied Mechanics, 1998, 68, 597-612.

73. Morozov N. F., Petrov Y. V. On the macroscopic parameters of brittle fracture. Arch. Mech. Int. J., 1996, 48, 825-833.

74. De Bremaecker J-C., Ferris M. A., Ralph D. Compressional fractures considered as contact problems and mixed complementarity problems. Eng. Fracture Mech., 2000, 66, 287-303.

75. Mogilevskaya S.G., Rothenburg L., Dusseault M.B. Growth of pressure-induced fractures in the vicinity of a wellbore. Int. J. Fracture, 2000, 104, L.25-L30.

76. Dobroskok A. A., Linkov A. M., Myer L., Roegiers J-C. On a new approach in micromechanics of solids and rocks. Mechanics in the National Interest. Proc. of the $38^{\text {th }}$ US Rock Mechanics Symposium. eds. Tinucci \& Heasley, Swets \& Zeitlinger Lisse. 2001, 1185-1190.

77. Koshelev V.F., Ghassemi A. Numerical modeling of stress distribution and crack trajectory near a fault or a natural fracture. Soil and Rock America 2003; $39^{\text {th }}$ US Rock Mechanics Symposium. P. J. Culligan, H. H. Einstein, A. J. Whittle (eds), Cambridge, Mass., 2003, 931-936. 
78. Dobroskok A.A. Numerical Simulation of Constitutive Equations for a Medium with Cracks and Contact Interaction. Ph. D. Thesis. Institute for Problems of Mechanical Engineering of Russian Academy of Sciences, Saint-Petersburg, 2003 (in Russian).

79. Dyskin A. V., Germanovich L. N., Ustinov K. B. A 3-D model of wing crack growth and interaction. Eng. Fracture Mech., 1999, 63, 81-110.

80. Linkov A. M. 1994. Dynamic Phenomena in Mines and the Problem of Stability. Int. Soc. Rock Mech., Lisboa, Cedex, Portugal.

81. Ingraffea A. R., Heuze F. E. Finite element models for rock fracture mechanics. Int. J. for Numerical and Analytical Methods in Geomechanics, 1980, 4, 25-43.

82. Germanovich L. N., Ring L. M., Carter B. J. et al. Simulation of crack growth and interaction in compression. Proc. $8^{\text {th }}$ Congress on Rock Mechanics. T. Fujii (ed.). Balkema, Rotterdam, 1995, 1, 219-226.

83. Cotterell B., Rice J. R. Slightly curved or kinked cracks. Int. J. Fracture, 1980, 16, 155-168.

84. Linkov, A.M. 2002. Boundary Integral Equations in Elasticity Theory. DordrechtBoston-London, Kluwer Academic Publishers, 2002.

85. Dobroskok A. A. Crack growth near a free surface. Mathematical Modeling in Solid Mechanics. Boundary \& Finite Elements Methods. Proc. $20^{\text {th }}$ Int. Conference. V. A. Postnov (ed.). St-Petersburg, 2003, 2, 184-187.

86. Linkov A.M., and S.G.Mogilevskaya. 1998. Complex hypersingular BEM in plane elasticity problems. In Proc. Singular Integrals in Boundary Element Method. eds. Sladek\&Sladek, 299-364. Comp. Mech. Publications Southampton.

87. Lam K.Y., and M.P. Cleary. 1984. Slippage and re-initiation of (hydraulic) fractures at frictional interfaces. Int. J. Numer. Analyt. Meth. Geomech. 8: 589-604.

88. Thiercelin M., J.C. Rogiers, T.J. Boone, and A.R. Ingraffea. 1987. An investigation of the material parameters that govern the behavior of fractures approaching rock interfaces. Proc. Of Sixth Int. Congress on Rock Mech. 1: 263-269. Montreal: Canada.

89. Renshaw C.E., and D.D. Pollard. 1995. An experimentally verified criterion for propagation across unbounded frictional interfaces in brittle, linear elastic materials. Int. J. of Rock Mech. and Min. Sci.\& Geomech. Abstr. 32(3): 237-249. 


\section{Coupled Poro-thermoelastic Processes}

Drilling a wellbore causes temperature variations in the formation. In a fluid-saturated porous rock, thermal loading can significantly alter the surrounding stress and pore pressure fields. Thermal loading induces volumetric deformation because of thermal expansion/contraction of both the pore fluid and the rock solid. If the rock is heated, expansion of the fluid can lead to a significant increase in pore pressure when the pore space is confined. The tendency is reversed in the case of cooling. Therefore, the timedependent poromechanical processes should be fully coupled to the transient temperature field. This can be studied in the framework of poro-thermoelasticity.

Aside from poromechanical and thermal phenomena, chemical processes are also active in a geothermal reservoir. These processes occur on various time scales and the significance of their interaction and coupling is dependent upon the problem of interest. For example, during drilling operations there is a strong coupling between thermal and poro-mechanical effects that has significant impact on wellbore failure (shear and tensile failure). This is because usually the time required for drilling a length equal to five times the hole's radius is much smaller than the characteristic time $\left(a^{2} / c^{\mathrm{f}}\right)$, where $a$ is the radius of the well and $c^{\mathrm{f}}$ is the fluid diffusivity. On the other hand, during stimulation by hydraulic fracturing the evolution of the fluid-rock mechanics coupling is rapid (on the scale of minutes to hours) compared to thermal processes, thus the thermal effects have little effect on the fluid-mechanical processes involved in fracture propagation. However, during long term injection operations (time scale of weeks to years) or, when the fluid pressure is below the level necessary to hydraulically derive a fracture, the thermomechanical coupling can no longer be neglected. In fact, thermo-mechanical processes are also coupled to chemical processes. Accordingly, different levels of coupling are considered herein when studying wellbore failure, fracture propagation, and fluid circulation.

In order to study the pore pressure and stress field under combined thermal and poromechanical loading, the theory of poro-thermoelasticity [1-3] is used.

\subsection{Governing Equations of Poro-thermoelasticity}

In the theory of poro-thermoelasticity, it is assumed that rock (and fluid) deformation has negligible influence on the temperature field. Heat convection can usually be neglected because of the extremely low fluid flow velocity in a rock such as granite. So that, for rocks with low permeability, heat conduction dominates the heat transfer process [4]. This means that the temperature and heat flux can be calculated separately without the contribution of pore pressure and stresses. The relationships of these three main components in poro-thermoelasticity are indicated in Figure 3.1. 


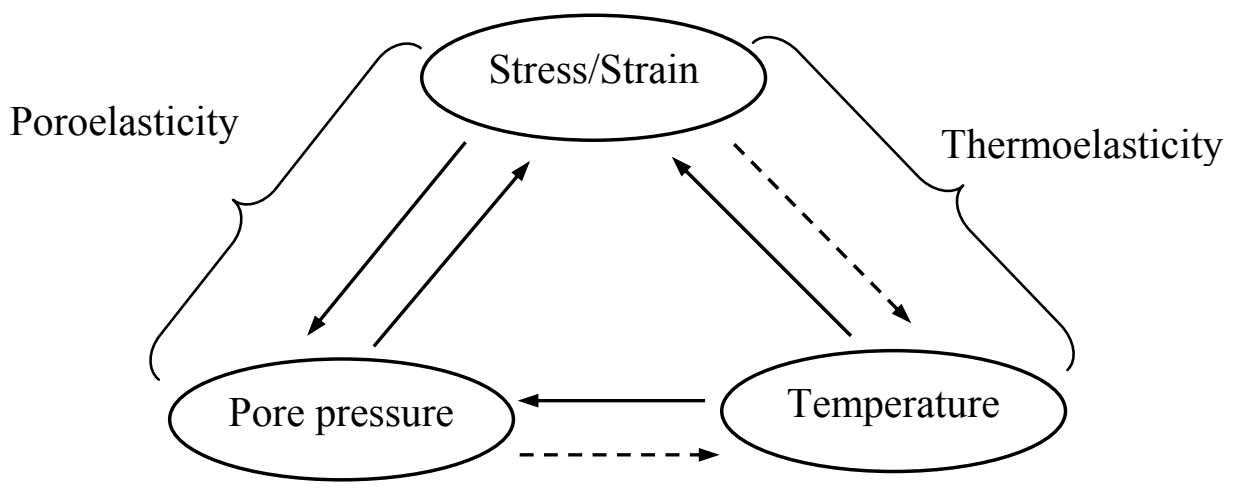

Figure 3.1. Illustration of coupling between poro-thermo-mechanical processes.

The following assumptions are made in this work:

- Homogeneous, isotropic, infinite rock

- Constant material parameters

- Transient fluid flow governed by Darcy's Law

- Transient linear heat conduction

The governing equations for poro-thermoelasticity are briefly reviewed next; these consist of the constitutive equations, transport laws, and balance laws.

\subsubsection{Constitutive Equations}

In isotropic poro-thermoelasticity, the constitutive equations can actually be separated into a deviatoric response and a volumetric one. The latter includes volumetric response of the solid matrix and the fluid. Deviatoric response is given by:

$$
\varepsilon_{i j}=\frac{\sigma_{i j}}{2 G}, \quad \text { for } i \neq j
$$

where $\varepsilon_{i j}$ denotes the components of the deviatoric strain tensor, $\sigma_{i j}$ denotes the components of the deviatoric strain stress tensor, and $G$ is the shear modulus. Throughout this report, subscript indices $i$ and $j$ have values in the range $\{1,2\}$ and the summation convention is used over repeated indices unless otherwise indicated.

The volumetric response of the solid contains both hydraulic and thermal coupling terms:

$$
\varepsilon_{k k}=\frac{\sigma_{k k}}{3 K}+\frac{\alpha p}{K}+\beta_{s} T
$$


where $\varepsilon_{k k}$ is volumetric strain, also denoted as $\varepsilon, \frac{\sigma_{k k}}{3}$ is volumetric stress (mean stress), $p$ is pore pressure change, $T$ the is temperature change. The constant $K$ is the rock's bulk modulus; $\alpha$ is Biot's [5] effective stress coefficient and can be computed using $\alpha=1-\left(K / K_{s}\right)$, where $K_{s}$ is the bulk modulus of solid grains; $\beta_{s}$ is the volumetric thermal expansion coefficient of the bulk solid under constant pore pressure and stress. Note that without the pore pressure term and temperature term, equation (3.2) degenerates to the classical elastic relation. Equation (3.2) can also be written in terms $\mathrm{f}$ stresses:

$$
\sigma_{i j}=2 G \varepsilon_{i j}+\frac{2 G v}{1-2 v} \varepsilon_{k k} \delta_{i j}-\alpha p \delta_{i j}+K \beta_{s} T \delta_{i j}
$$

in which $v$ is Possion's ratio.

The volumetric response of the fluid can be written as:

$$
\zeta=\frac{\alpha}{3 K} \sigma_{k k}+\frac{\alpha p}{B K}-n\left(\beta_{f}-\beta_{s}\right) T
$$

where $\zeta$ is the variation of the fluid content per unit volume of the porous material [5], $B$ is Skempton's pore pressure coefficient, $\beta_{f}$ is volumetric thermal expansion coefficient of the fluid and $n$ is porosity. The parameter $B$ controls the increase in pore pressure due to an increase of mean stress under isothermal undrained condition $(\zeta=0)$.

Equation (3.4) can also be written in term of pore pressure:

$$
p=M\left(\zeta-\alpha \sigma_{k k}+\beta_{m} T\right)
$$

where $M$ is the Biot modulus given as $M=\frac{B K}{\alpha(1-B \alpha)}, \beta_{m}$ is hydro-thermal expansion coefficient given as $\beta_{m}=\alpha \beta_{s}+n\left(\beta_{f}-\beta_{s}\right)$.

\subsubsection{Transport Laws}

The transient fluid flow in porous rocks is governed by the well-known Darcy's law, which can be described as:

$$
q_{i}=-\kappa p_{, i}
$$

where $q_{i}$ is the fluid flux (units of fluid volume per unit area); $\kappa=k / \mu$ ( $k$ is the intrinsic permeability having dimension of length squared, and $\mu$ the fluid dynamic viscosity).

The heat flow is governed by Fourier law, which is written as:

$$
q_{i}^{T}=-\kappa^{T} T_{, i}
$$

where $q_{i}^{T}$ is the heat flux, $\kappa^{T}$ is the thermal conductivity. One can see that the transport laws for fluid flow and heat flow are analogous to each other. 


\subsubsection{Balance Laws}

For local stress balance, standard considerations of static equilibrium lead to the equilibrium equations used in elasticity:

$$
\sigma_{i j, j}=0
$$

Considerations of mass conservation for a compressible fluid yield the local continuity equation:

$$
\frac{\partial \zeta}{\partial t}+q_{i, i}=0
$$

\subsection{Field Equations of Poro-thermoelasticity}

From the constitutive, balance, and transport laws, the field equations can be derived for temperature, $T$, displacement, $u_{i}$, and pore pressure, $p$ :

Navier Equation:

$$
G \nabla^{2} u_{i}+\frac{1}{3}(G+3 K) \varepsilon_{, i}=\alpha p_{, i}+K \beta_{s} T_{, i}
$$

Diffusion equation for pore pressure $p$ :

$$
\kappa \nabla^{2} p=\frac{1}{M} \frac{\partial p}{\partial t}+\alpha \frac{\partial \varepsilon}{\partial t}-\beta_{m} \frac{\partial T}{\partial t}
$$

Diffusion equation for temperature $T$ :

$$
c^{T} \nabla^{2} T=\frac{\partial T}{\partial t}
$$

In the above equations, $u_{i}$ denotes the components of the solid displacement vector, $\varepsilon_{i j}$ the total strain tensor, $p$ the pore pressure change, and $T$ the temperature change. The constant $c^{T}$ represents thermal diffusivity. As mentioned above, heat transfer is calculated separately because stress and pressure changes do not significantly alter the temperature field. Also, note that convective heat transport is neglected.

Ten independent parameters are needed for poro-thermoelastic theory, they are $\{K, G, \alpha$, $\left.B, \kappa, \beta_{s}, \beta_{f}, c^{T}, \kappa^{T}, \gamma_{f}\right\}$. Among them, five parameters $\{K, G, \alpha, B, \kappa\}$ are from poroelasticity, with $\{K, G\}$ as the pure elastic parameters. 


\subsubsection{Field Equations for poroelasticity and thermoelasticity}

Poroelasticity and thermoelasticity can be considered as two special cases of porothermoelasticity. Derivations of field equations for poroelasticity can be found in the literature e.g., [6]. These equations can also be obtained from the poro-thermoelastic ones by neglecting the thermal effect, doing so in equations (3.10) and (3.11), one can get the field equations for poroelasticity:

\section{Navier Equation:}

$$
G \nabla^{2} u_{i}+\frac{1}{3}(G+3 K) \varepsilon_{, i}=\alpha p_{, i}
$$

\section{Diffusion equation $p$ :}

$$
\kappa \nabla^{2} p=\frac{1}{M} \frac{\partial p}{\partial t}+\alpha \frac{\partial \varepsilon}{\partial t}
$$

Similarly, neglecting the pore pressure term from the field equations of porothermoelasticity will result in field equations for thermoelasticity:

\section{Navier Equation:}

$$
G \nabla^{2} u_{i}+\frac{1}{3}(G+3 K) \varepsilon_{, i}=K \beta_{s} T_{, i}
$$

Diffusion equation for temperature, $T$, would be the same as the poro-thermoelastic equation because actually no pore pressure and stress effects are considered. The analogies between poroelasticity and thermoelasticity have been discussed in references [7-8].

\subsection{Poro-thermoelastic Boundary Element Method}

A few analytical procedures have been developed and used to solve geomechanics problems of interest involving coupled thermal and poromechanical problems [9-10]. However, many problems formulated within the framework of poro-thermoelasticity are not amenable to analytical treatment and need to be solved numerically. The boundary element method (BEM) or the boundary integral equation formulation has been used extensively for the poroelastic and thermoelastic problems e.g., [11]. The advantage of the method is that it reduces the problem dimensionality by one, thereby reducing the computational efforts significantly.

Boundary element method (BEM) is a powerful numerical tool for solving systems governed by linear partial differential equations [12]. BEM is based on fundamental solutions, which are analytical solutions corresponding to some sort of singular impulse at a point in an infinite region. For example, in solid mechanics, the impulse can represent a point force applied within an elastic solid. The impulse could also represent a point fluid source, a point heat source or a displacement discontinuity in different problems. The fundamental solutions are also called singular solutions because, 
mathematically speaking, they are well behaved everywhere in the region except at the point of the impulse, where there is a mathematical singularity.

Partial differential equations plus certain conditions specified on the boundary of the region of interest $R$, enclosed within a boundary $\Gamma$, defines a boundary value problem. In contrast to the finite difference and finite element methods which make approximations on the whole region $R$, BEM makes approximations only on the boundary $\Gamma$ by dividing it into $N$ elements, as shown in Figure 3.2, after [13]. Therefore, a system of $N$ linear algebraic equations can be formed. Once these equations have been solved, the solution at any point in $R$ can be constructed. The boundary-only discretization significantly simplifies modeling. The system of equations need to be solved is much smaller than the system needed to solve the same boundary value problem by finite element method. However, this smaller system of equations is no longer sparse as each singular impulse plays a part in every equation (Crouch and Starfield, 1983). Another major advantage is that BEM can generate solutions at any point in the region $\mathrm{R}$, instead of a number of fixed mesh points in finite element method. This is because BEM exploits analytical solutions that hold true for the whole region. Therefore, BEM is potentially more accurate than finite element method, where approximations are made in every subdivision of $R$ [13].

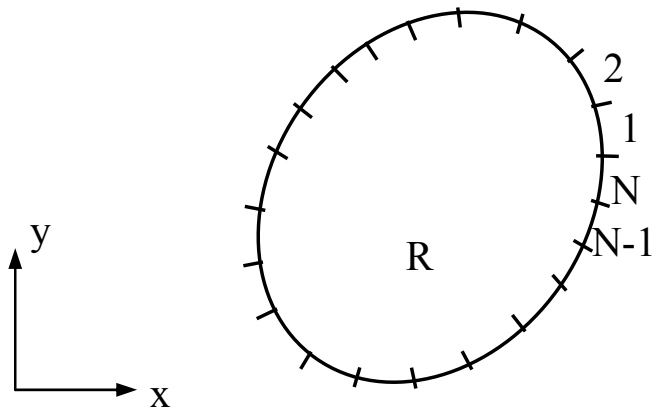

Figure 3.2. Discretization in the boundary element method.

It is worth noting that there two general methods of formulating the BE equations, the direct and the indirect methods. The direct method is based on the generalized Green's theorem, which is sometimes expressed in the form of an energy reciprocity theorem [14]. Solution of the integral equations for the elements into which a boundary is discretized directly yields the desired values of the unknown variables on the boundary.

In the indirect method, singular impulses (e.g., point force, heat source, fluid source, or displacement discontinuity) are distributed on the elements along the boundary so that the combined effects of all the impulses satisfy the prescribed boundary conditions.

The indirect form as applied to the problems of our interest has two sub-formulations, namely, the fictitious stress method (FSM) and the displacement discontinuity method (DDM). The fictitious stress method is based on the analytic solution of a point force in an infinite solid. It is a versatile method of modeling underground openings of arbitrary shape. The displacement discontinuity method makes use of the fundamental solution for a constant discontinuity of displacement in an infinite solid. The displacement jump inherent in the fundamental solution of displacement discontinuity method are not fictitious quantities, this makes it a natural choice for modeling fractures. The fictitious 
stress method is not suitable for such problems, because the effects of elements placed along one crack surface are indistinguishable from the effects of elements placed along the other surface [13].

Although the coupled diffusion-deformation problems are essentially three-dimensional (3-D), they can be analyzed in 2-D using the concept of plane strain as long as the length of the excavation is much larger than its dimension in the plane perpendicular to its axis. The plane strain concept can also be used when the long axis of the opening is not in the direction of a principal stress [11,15] which is often practiced in engineering. In this work, a plane strain 2-D indirect boundary element model is developed and applied to borehole and fracture problems.

\subsubsection{Displacement discontinuity method for poro-thermoelasticity}

The displacement discontinuity (DD) method is based on the fundamental solution for the problem of constant normal and shear discontinuities in displacement over a finite line segment in the $\mathrm{x}$, $\mathrm{y}$ plane of an infinite elastic solid in plane strain, as shown in Figure 3.3 (after 13]). The line segment is chosen to occupy a certain portion: $|x| \leq a, y=0$, Consider this segment to be a line crack with two surfaces, one is on the positive side of $y=0$, denoted $y=0^{+}$, and the other is on the negative side, denoted $y=0^{-}$.

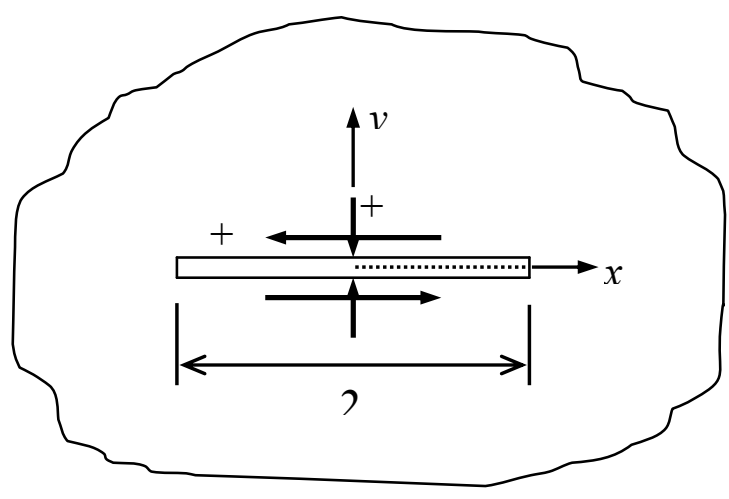

Figure 3.3. Constant normal and shear displacement discontinuity.

From one side of the line segment to the other, the displacements undergo a constant specified change in value $D_{i}=\left(D_{x}, D_{y}\right)$. The displacement discontinuity, $D_{i}$ is defined as the difference in displacement between the two sides of the segment as follows:

$$
\begin{aligned}
& D_{x}=u_{x}\left(x, 0^{-}\right)-u_{x}\left(x, 0^{+}\right) \\
& D_{y}=u_{y}\left(x, 0^{-}\right)-u_{y}\left(x, 0^{+}\right)
\end{aligned}
$$

The elastic solution to this problem is given by Crouch and Starfield [13]. The displacements and the stress components are then defined everywhere with functions of $D_{x}$ and $D_{y}$. The corresponding fundamental solutions for continuous impulses required in 
displacement discontinuity method are the stresses and pore pressures due to DD, fluid source, and heat sources: $\sigma_{i j k}^{c d}, \sigma_{i j}^{c s}, \sigma_{i j}^{c h}, p_{i}^{c d}, p^{c s}, p^{c h} \cdot \sigma_{i j k}^{c d}, \sigma_{i j}^{c s}, p_{i}^{c d}$, and $p^{c S}$ are poroelastic terms, and have been given in [20].

In the DD method, temperature, pore pressure, and stresses fields on the boundary can be approximated at any given time, $t$, by the following methodology (note that the heat equation is not coupled to others, thus it can be solved independently first):

1. Distribute point DDs, fluid sources and heat sources on the boundary elements.

2. Temperature at each element is the sum of all temperatures caused by all heat sources taking place at time $\tau \leq t$. (The temperature field is decoupled from pore pressure and stresses because fluid sources and point DD do not contribute to temperature change.) The strengths of heat sources, which are functions of time, are calculated such that they satisfy the temperature boundary conditions.

3. Pore pressure at each element is the sum of all pore pressures caused by all point forces, fluid sources and heat sources taking place at time $\tau \leq t$. The strengths of heat sources are known from step (2).

4. Stresses at each element are the sum of all corresponding stresses induced by all $\mathrm{DD}$, fluid sources and heat sources taking place at time $\tau \leq t$. The strengths of heat sources are known from step (2).

5. To satisfy the boundary conditions of pore pressure and stresses, strengths of DD and fluid sources can be solved since the contributions from heat sources have been determined. The strengths of DD and fluid sources are also functions of time.

Then, one can obtain the stresses, pore pressure, and temperature at any point using the history of the strengths of heat sources, fluid sources and DD forces along the boundary. The determination of this history requires the solution of a set of three singular integral equations:

$$
\sigma_{i j}(\mathbf{x}, t)=\int_{a}^{t} \Gamma_{\Gamma}\left\{\begin{array}{c}
\sigma_{i j k}^{i d}(\mathbf{x}, \chi ; t-\tau) D_{k}(\chi, \tau) \\
+\sigma_{i j}^{i s}(\mathbf{x}, \chi ; t-\tau) \phi(\chi, \tau) \\
+\sigma_{i j}^{i h}(\mathbf{x}, \boldsymbol{\chi} ; t-\tau) \varphi(\chi, \tau)
\end{array}\right\} d \Gamma(\chi) d \tau
$$




$$
\begin{aligned}
& p(\mathbf{x}, t)=\int_{0}^{t} \int_{\Gamma}\left\{\begin{array}{l}
p_{k}^{i d}(\mathbf{x}, \boldsymbol{\chi} ; t-\tau) D_{k}(\boldsymbol{\chi}, \tau) \\
+p^{i s}(\mathbf{x}, \boldsymbol{\chi} ; t-\tau) \phi(\boldsymbol{\chi}, \tau) \\
+p^{i h}(\mathbf{x}, \boldsymbol{\chi} ; t-\tau) \varphi(\boldsymbol{\chi}, \tau)
\end{array}\right\} d \Gamma(\boldsymbol{\chi}) d \tau \\
& T(\mathbf{x}, t)=\int_{0}^{t} \int_{\Gamma}\left\{T^{i h}(\mathbf{x}, \boldsymbol{\chi} ; t-\tau) \varphi(\boldsymbol{\chi}, \tau)\right\} d \Gamma(\boldsymbol{\chi}) d \tau
\end{aligned}
$$

These are called integral equations because the unknowns appear inside the integral sign. Aside from the heuristic approach based on the principle of superposition, the integral equations can be obtained using a rigorous approach based on the reciprocal theorem [16]. In the above equations, $\Gamma$ is the boundary; $\mathbf{x}$ and $\chi$ are the two-dimensional coordinate vectors. $\sigma_{i j}(\mathbf{x}, t)$ denotes the stress component at $\mathbf{x}$ and at time $t$. The influence function $\sigma_{i j k}^{i d}(\mathbf{x}, \chi ; t-\tau)$ represents the stress component $\sigma_{i j}(\mathbf{x}, t)$ due to an instantaneous unit point DD, located at $\chi$ and taking place at time $\tau$. Similarly, the symbols $\sigma_{i j}^{i s}(\mathbf{x}, \boldsymbol{\chi} ; t-\tau)$ and $\sigma_{i j}^{i h}(\mathbf{x}, \chi ; t-\tau)$ represent the stress components induced by an instantaneous fluid source and an instantaneous heat source, respectively. $p_{k}^{i d}, p^{i s}$ and $p^{i h}$ are the pore pressure induced by an instantaneous unit point $\mathrm{DD}$, fluid source and heat source; the latter are given by [17]. $T^{i h}$ is the temperature induced by a instantaneous unit heat source. $D_{k}, \phi(\chi, \tau)$, and $\varphi(\chi, \tau)$ are the strengths of the point DD, fluid source and heat source, respectively. Superscripts " $i d$ ", "is" and "ih" denote instantaneous displacement discontinuity, fluid source and heat source, respectively. Subscript $i, j, k$ have a range of $1-2$, corresponding to the two spatial directions. It can be seen that the temperature part remains the same in the two methods, because temperature is calculated separately while pore pressure and stress are fully coupled.

\section{Numerical Implementation:}

Numerical implementation of the boundary integral equations of transient porothermoelasticity requires spatial and temporal discretization. Spatial discretization is achieved by dividing the boundary of the problem into a number of elements and replacing the integrals over the boundary by a sum of integrals over these elements. Temporal discretization is realized by dividing the time domain into a number of time increments and utilizing a time marching scheme. In the present implementation, the following approximations are made: the boundary elements are straight-line segments; the singular impulses (point force, displacement discontinuity, fluid source, heat source) are located at the midpoint of each element; the intensity of the impulses is constant over each element, and the time increments, $\Delta t$, are constant. 
Suppose $p+1$ is the number of time increments used, then the boundary integral equations for induced stresses, pore pressure and temperature can be rewritten as:

$$
\begin{aligned}
& \sigma_{i j}=\sum_{\ell=0}^{p} \int_{\Gamma}\left\{\sigma_{i j k}^{c d} D_{k}^{\ell}+\sigma_{i j}^{c s} \phi^{\ell}+\sigma_{i j}^{c h} \varphi^{\ell}\right\} d \Gamma \\
& p=\sum_{\ell=0}^{p} \int_{\Gamma}\left\{p_{k}^{c p} D_{k}^{\ell}+p_{i j}^{c s} \phi^{\ell}+p_{i j}^{c h} \varphi^{\ell}\right\} d \Gamma \\
& T=\sum_{\ell=0}^{p} \int_{\Gamma}\left\{T_{i j}^{c h} \varphi^{\ell}\right\} d \Gamma
\end{aligned}
$$

where $D_{k}^{\ell}, \phi^{\ell}$ and $\varphi^{\ell}$ are strengths of continuous point DD, fluid source and heat source in time increment $\ell$. Suppose $N$ is the number of elements used to discretize the boundary. The spatial integrals over the boundary are replaced by a sum of integrals over these elements. Then, the induced stresses, pore pressure, and temperature on element $m$ due to a constant spatial distribution of continuous DDs, fluid sources and heat sources on element $r$ are given by:

$$
\begin{aligned}
& \sigma_{i j}^{m}=\sigma_{i j k}^{c p} D_{k}^{r}+\sigma_{i j}^{c s} \phi^{r}+\sigma_{i j}^{c h} \varphi^{r} \\
& p^{m}=p_{k}^{c d} D_{k}^{r}+p^{c s} \phi^{r}+p^{c h} \varphi^{r} \\
& T_{i j}^{m}=T^{c h} \varphi^{r}
\end{aligned}
$$

where $D_{k}^{r}, \phi^{r}$ and $\varphi^{r}$ are strengths of continuous point DD, fluid source and heat source on element $r$. The superscripts $m$ and $r$ refer to the influenced and influencing elements, respectively. Influence coefficients: $\sigma_{i j k}^{c d}, \sigma_{i j}^{c s}, \sigma_{i j}^{c h}, p_{i}^{c d}, p^{c s}, p^{c h}$ and $T^{c h}$ now denote the influences of the element $r$, which are obtained by integrating the fundamental solutions for continuous point impulses over the influencing element $r$. The spatial integration over the straight-line constant elements is a relatively easy process in a twodimensional system.

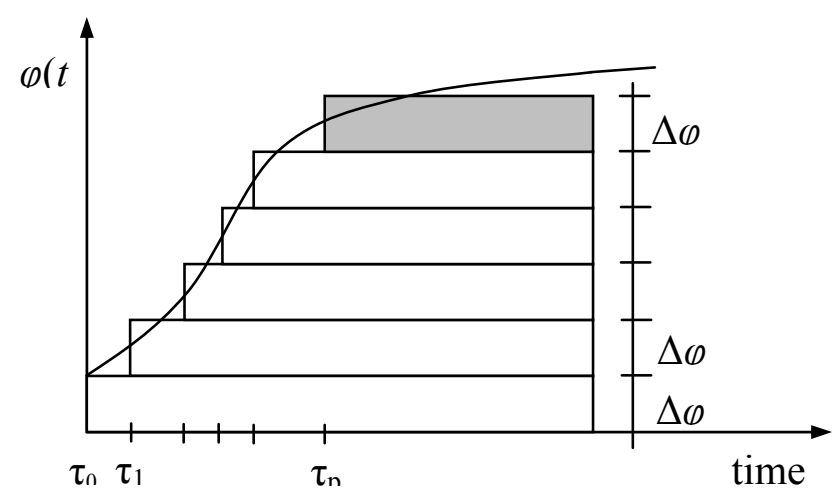

Figure 3.4. Time marching scheme for a continuous heat/fluid source or DD.

There are different approaches to the temporal solution of the problem. One approach is to solve the problem at the end of a time step, and then use the results as the initial 
conditions for the next time step, marching forward in time. The disadvantage of this method is that it requires discretizing the spatial domain of the problem. The second approach is a time marching technique which solves the problem at the end of a time step, but keeps a solution history [19]. This allows for strengths of singular impulses to vary with time. It involves incrementing the strengths of singular impulses at each time step and including the influence of all previous increments. This technique eliminates the need for internal discretization of the spatial domain. But, it has the disadvantage that the coefficient matrix must be stored to be used as required. The scheme is illustrated with the heat source $\varphi(t)$ as an example, in Figure 3.4; after [20].

The implementation of this time marching scheme is possible because it is the time interval between the loading and receiving that affects the response rather than the absolute times. This is the so-called "time translation" property of the fundamental solutions. For example, the stress at a point $\mathbf{x}$ and time $t$ due to a heat source taking place at point $\chi$ and at time $\tau$ is equal to the stress at point $\mathbf{x}$ and time $t$ - $\tau$ due to a heat source occurring at time zero at the point $\chi$. That is:

$$
\sigma_{i j}^{c h}(\mathbf{x}, t ; \chi, \tau)=\sigma_{i j}^{c h}(\mathbf{x}, t-\tau ; \chi, 0)
$$

Due to this property of the fundamental solutions, the evaluation time and loading time can be shifted along the time axis without affecting the values of the fundamental solutions. Therefore, the influence coefficient can be calculated only once during the calculation history.

The induced stresses, pore pressure and temperature of element $m$ are given by:

$$
\begin{aligned}
\sigma_{i j}^{m}(\mathbf{x}, t) & =\sum_{r=1}^{N}\left[\sigma_{i j k}^{c d}\left(\mathbf{x}, \boldsymbol{\chi}, t-\tau_{p}\right) \Delta D_{k}^{r p}+\sigma_{i j}^{c s}\left(\mathbf{x}, \boldsymbol{\chi}, t-\tau_{p}\right) \Delta \phi^{r p}+\sigma_{i j}^{c h}\left(\mathbf{x}, \boldsymbol{\chi}, t-\tau_{p}\right) \Delta \varphi^{r p}\right] \\
& +\sum_{\ell=0}^{p-1} \sum_{r=1}^{N}\left[\sigma_{i j k}^{c d}\left(\mathbf{x}, \boldsymbol{\chi}, t-\tau_{\ell}\right) \Delta D_{k}^{r \ell}+\sigma_{i j}^{c s}\left(\mathbf{x}, \boldsymbol{\chi}, t-\tau_{\ell}\right) \Delta \phi^{r \ell}+\sigma_{i j}^{c h}\left(\mathbf{x}, \boldsymbol{\chi}, t-\tau_{\ell}\right) \Delta \varphi^{r \ell}\right] \\
p^{m}(\mathbf{x}, t) & =\sum_{r=1}^{N}\left[p_{k}^{c d}\left(\mathbf{x}, \boldsymbol{\chi}, t-\tau_{p}\right) \Delta D_{k}^{r p}+p^{c s}\left(\mathbf{x}, \boldsymbol{\chi}, t-\tau_{p}\right) \Delta \phi^{r p}+p^{c h}\left(\mathbf{x}, \boldsymbol{\chi}, t-\tau_{p}\right) \Delta \varphi^{r p}\right] \\
& +\sum_{\ell=0}^{p-1} \sum_{r=1}^{N}\left[p_{k}^{c d}\left(\mathbf{x}, \boldsymbol{\chi}, t-\tau_{\ell}\right) \Delta D_{k}^{r \ell}+p^{c s}\left(\mathbf{x}, \boldsymbol{\chi}, t-\tau_{\ell}\right) \Delta \phi^{r \ell}+p^{c h}\left(\mathbf{x}, \boldsymbol{\chi}, t-\tau_{\ell}\right) \Delta \varphi^{r \ell}\right] \\
T^{m}(\mathbf{x}, t) & =\sum_{r=1}^{N}\left[T^{c h}\left(\mathbf{x}, \boldsymbol{\chi}, t-\tau_{p}\right) \Delta \varphi^{r p}\right]+\sum_{\ell=0}^{p-1} \sum_{r=1}^{N}\left[T_{i j}^{c h}\left(\mathbf{x}, \boldsymbol{\chi}, t-\tau_{\ell}\right) \Delta \varphi^{r \ell}\right]
\end{aligned}
$$

where $p+1$ is the number of time increments, $N$ is the number of elements. $\Delta D_{k}^{r p}$, and $\Delta \phi^{r \ell}$, are the increments of strengths of certain kind of continuous impulse occurring on element $r$. Subscript $k$ takes value from $\{1,2\}$, which denotes the two spatial directions. The influence coefficient $\sigma_{i j k}^{c d}$ and $p_{k}^{c d}$ are obtained by spatial integration of fundamental solutions of a displacement discontinuity on the influencing element $r$. Equation (3.28), (3.29) and (3.30) constitute a set of linear algebraic equations with the unknowns are the 
increments of the singular impulses. They can be solved by applying the boundary conditions.

\subsubsection{Natural fracture deformation using poro-thermoelastic DD}

In this part of the work, we only consider those fractures with two rough surfaces to contact each other and the fluid flow can flow through the void spaces between them as illustrated in Figure 3.5. The fracture can deform normally and laterally to the fracture surface as the stress acting on it changes.

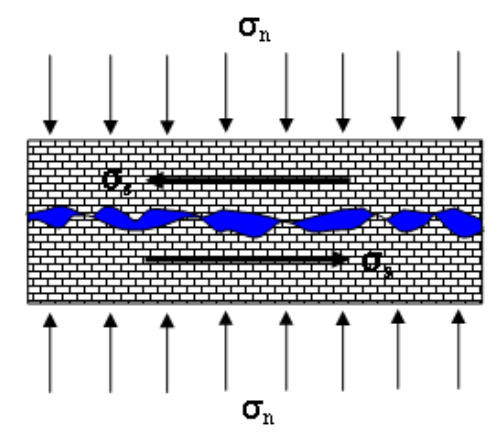

Figure 3.5. A fluid filled fracture subject to normal and shear stress.

Bandis et al.[21] presented a hyperbolic model for the normal deformation of fracture based on a large body of experimental data.

$\sigma_{n}^{\prime}=\frac{D_{n}}{a-b D_{n}}$

where $\sigma_{n}^{\prime}$ is the effective normal stress and $D_{n}$ is the normal closure of fracture; $a$ and $b$ are constants and related with the experimentally determined parameters initial normal stiffness $\left(K_{n i}\right)$ and the maximum possible closure $\left(D_{\text {nmax }}\right)$ as $a=1 / K_{n i}$ and $a / b=D_{n \text { max }}$.

Eq. (31) can be rewritten by substituting $K_{n i}$ and $D_{n \max }$ for $a$ and $b$.

$\sigma_{n}^{\prime}=\frac{K_{n i} D_{n}}{1-D_{n} / D_{n \max }}$

The normal stiffness $\left(K_{n}\right)$ is therefore derived according to Eq. (32) as a function of $D_{n}$ or $\sigma_{n}^{\prime}$ :

$$
K_{n}=\frac{\partial \sigma_{n}^{\prime}}{\partial D_{n}}=\frac{K_{n i}}{\left(1-D_{n} / D_{n \max }\right)^{2}} \quad \text { (33) Or } K_{n}=\frac{K_{n i}}{\left[1-\sigma_{n}^{\prime} /\left(K_{n i} D_{n \max }+\sigma_{n}^{\prime}\right)\right]^{2}}
$$

In the elastic range, the change of shear stress has a linear relationship with the change of shear displacement:

$$
\Delta \sigma_{s}=K_{s} \Delta D_{s}
$$


where $K_{s}$ is the shear stiffness. The fracture slips when the shear stress exceeds the shear strength $\left(\sigma_{c}\right)$ of the fracture defined according to the Mohr-Coulomb failure criterion:

$$
\sigma_{c}=\sigma_{n}^{\prime} \tan \phi^{\prime}+c_{o}
$$

where $\phi^{\prime}$ is the frictional angle and $c_{0}$ is the cohesion strength. The two rough surfaces slide each other and cause an increase in apertures which is known as frictional dilation. We used an approximate linear relation for the aperture increase $\left(\Delta D_{n \text {-dilation }}\right)$ due to shear movement.

$\Delta D_{n-\text { dilation }}=-\Delta D_{s} \tan \psi_{\text {dilation }}$

where $\psi_{\text {dilation }}$ is the dilation angle.

\section{Numerical implementation}

Using the stress-displacement relationship of fracture, the normal and shear stress of the $k t h$ fracture are related with the normal and shear displacement of the kth fracture:

$\Delta \sigma_{n}^{k}-\Delta p^{k}=-K_{n}\left(\Delta D_{n}^{k}+\tan \psi_{\text {dilation }} \Delta D_{s}^{k}\right)$

$\Delta \sigma_{s}^{k}=K_{s} \Delta D_{s}^{k}$

The normal $\sigma_{n}^{k}$ and shear stress $\sigma_{s}^{k}$ and pore pressure $p^{k}$ are also related with the sum of the effects of the normal and shear displacement and interface flow of all of fractures in a fracture network according to the DD solutions:

$$
\begin{aligned}
& \Delta \sigma_{n}^{k}=\sum_{l=1}^{m} A^{l k} \Delta D_{n}^{l}+\sum_{l=1}^{m} B^{l k} \Delta D_{s}^{l}+\sum_{l=1}^{m} C^{l k} \phi^{l}+\sum_{l=1}^{m} D^{l k} \varphi^{l} \\
& \Delta \sigma_{s}^{k}=\sum_{l=1}^{m} E^{l k} \Delta D_{n}^{l}+\sum_{l=1}^{m} F^{l k} \Delta D_{s}^{l}+\sum_{l=1}^{m} K^{l k} \phi^{l}+\sum_{l=1}^{m} F^{l k} \varphi^{l} \\
& \Delta p^{k}=\sum_{l=1}^{m} L^{l k} \Delta D_{n}^{l}+\sum_{l=1}^{m} M^{l k} \Delta D_{s}^{l}+\sum_{l=1}^{m} N^{l k} \phi^{l}+\sum_{l=1}^{m} E^{l k} \varphi^{l} \\
& \Delta T^{k}=\sum_{l=1}^{m} R^{l k} \varphi^{l}
\end{aligned}
$$

where $A^{l k}, B^{l k}, C^{l k}, E^{l k}, F^{l k}, K^{l k}, L^{l k}, M^{l k}$ and $N^{l k}$ are the influence coefficients of the $l^{\text {th }}$ fracture on the $k^{\text {th }}$ fracture. Combining Eqs.(40)-(43) results $8 \mathrm{~m}$ equations for $\mathrm{m}$ fracture segments and $8 \mathrm{~m}$ unknowns $\left(\sigma_{n}, \sigma_{s}, D_{n}, D_{s}, p, \phi\right.$, and $\left.T, \varphi\right)$, therefore the $6 \mathrm{~m}$ unknowns can be obtained by numerically solving the $6 \mathrm{~m}$ linear equations.

The above formulations allow one to consider both the fracture propagation problem and natural fracture (joint) response to hydrothermal loads in e.g., injection extraction problems. Applications of these models will be shown in Chapter 5. 


\subsection{Poro-thermoelastic Fractured Rock Response to Injection/Extraction}

The porothermoelastic model for natural fractures is used to model a fractured geothermal reservoir and to study its response to injection/extraction operations. This is done by considering fluid flow and heat transport in a fracture network. To do so, we use the plain-strain approximation and consider a 2D horizontal section to reduce the solution geometry. The rock is assumed to be isotropic, homogeneous, and linearly elastic with constant permeability. Fluid flow in the fractures is viscous, and laminar such that lubrication flow theory applies and fluid properties are uniform throughout. The fracture aperture and joint deformation are significantly less than the joint lengths, and there are a large but finite number of joints. The total normal stress in the direction normal to the joint varies non-linearly, but in the shear direction, the joint stresses are proportional to deformation when the deformation are in the elastic range (using Mohr-Coulomb criterion). Flow in the joints is described by non-isothermal parallel plate flow model in which the flow rate is proportional to the pressure gradients and also depends on the cube of the apparent joint aperture.

\subsubsection{Fluid Flow \& Heat Transfer}

Assuming that Darcy's flow is applicable in the fracture network, the intrinsic fracture permeability $\left(k_{f f}\right)$ caused by the fracture aperture is defined as:

$k_{f f}=\frac{w_{f}^{2}}{12}$

where $w_{f}$ is the effective fracture aperture (twice the displacement discontinuity). On the other and, often the fracture permeability $\left(k_{f}\right)$ is expressed according to the cubic law:

$k_{f}=\frac{n_{f} w_{f}^{3}}{12}$

where $n_{f}$ is the fracture frequency — number of fractures per unit length.

The fluid balance equation in the fracture includes the flow from the connected fractures and the flux (interface flow) from and to the connected matrices.

$$
\left(\rho_{f} Q\right)_{, i}=-\frac{\partial\left(\rho_{f} n w_{f} \Delta L\right)}{\partial t}+\rho_{f} v+\rho_{f} Q_{s}
$$

where $Q$ is the flow rate in the fracture; $v$ is the flow velocity (linear flow rate) in $\mathrm{m} / \mathrm{s} ; Q_{s}$ is the production or injection term; $n$ is the fracture porosity or the ratio of actual fracture void volume $\left(V_{f}\right)$ to the effective fracture void volume for fluid flow $\left(V_{e f}\right)$, and $c_{f}$ is the fluid compressibility defined by: $\frac{\partial \rho_{f}}{\partial p}=c_{f} \rho_{f}$. The fluid diffusion equation in the fracture can be derived from Eq. (40). 


$$
\begin{aligned}
& \frac{k w_{f}}{\mu} \frac{\partial^{2} p}{\partial x^{2}}-v+n \Delta L w_{f} c_{f} \frac{\partial p}{\partial t}=Q_{\text {injection }}-Q_{\text {injection }} \quad \text { or: } \\
& \frac{k_{f f} w_{f}}{\mu} \frac{\partial^{2} p}{\partial x^{2}}=n w_{f} \Delta L c_{f} \frac{\partial p}{\partial t}-n \Delta L \frac{\partial D_{n}}{\partial t}+\Delta L v+Q_{s}
\end{aligned}
$$

Studies on the heat transfer suggest conduction and dispersion effect within the fracture are small, hence can be ignored [22]. Assuming that rock and fluid properties do not vary with temperature:, we obtain the following heat transport Eqn for the fracture equation:

$$
k_{T} w_{f} \frac{\partial^{2} T}{\partial x^{2}}+Q_{\text {interface }}=\rho_{w} c_{w}\left(n \Delta L w_{f} \frac{\partial T}{\partial t}+q_{\text {fluid }} \frac{\partial T}{\partial x}\right)+Q_{\text {injection }}
$$

$v$ is the interface flow between the fracture and matrix, $\rho_{\mathrm{w}}$ and $\mathrm{c}_{\mathrm{w}}$ is are fluid density and heat capacity, respectively, $\mathrm{k}_{\mathrm{T}}$ is the fluid thermal conductivity, and $\mathrm{q}_{\mathrm{f}}$ is the fluid velocity. The $\mathrm{Q}_{\text {inj }}$ in the heat transport equation represents the heat loss/gain at the wellbore wall, due to fluid injection. The heat transport in the matrix is assumed conductive and that heat transport due to fluid leak-off is not considered.

\subsection{Solution Method}

The fluid pressure change and heat flux induce fracture deformation and the fracture deformation influences the fluid pressure distribution but not the heat flux. The heat source (interface heat flow) is solved for the system using a given flow rate and temperature distribution. Then the fluid pressure, leak-off (interface fluid flow rate), normal and shear fracture displacement are solved simultaneously.

The displacement change and interface flow rate are time-dependent for practical applications. This is simulated using poro-thermoelastic DD with the time integration has going back to the initial homogeneous state (convolution integration). Therefore, all the previous increments of source strengths need to be included while integrating the effect of source strengths at each time step. The induced stress and pore pressure on the $i^{\text {th }}$ fracture element by the increments of source strengths are:

$$
\begin{aligned}
& \Delta \sigma_{n}^{i}(t)=\sum_{l=1}^{m} A^{l i}\left(t-\tau_{p}\right) \Delta D_{n}^{l p}+\sum_{l=1}^{m} B^{l i}\left(t-\tau_{p}\right) \Delta D_{s}^{l p}+\sum_{l=1}^{m} C^{l i}\left(t-\tau_{p}\right) \nu^{l p} \\
& \sum_{h=0}^{p-1} \sum_{l=1}^{m} A^{l i}\left(t-\tau_{h}\right) \Delta D_{n}^{l h}+\sum_{h=0}^{p-1} \sum_{l=1}^{m} B^{l i}\left(t-\tau_{h}\right) \Delta D_{s}^{l h}+\sum_{h=0}^{p-1} \sum_{l=1}^{m} C^{l i}\left(t-\tau_{h}\right) \nu^{l h} \\
& +\sum_{h=0}^{p} \sum_{l=1}^{m} R^{l i}\left(t-\tau_{h}\right) v_{h}^{l h}
\end{aligned}
$$




$$
\begin{aligned}
& \Delta \sigma_{s}^{i}(t)=\sum_{l=1}^{m} E^{l i}\left(t-\tau_{p}\right) \Delta D_{n}^{l p}+\sum_{l=1}^{m} F^{l i}\left(t-\tau_{p}\right) \Delta D_{s}^{l p}+\sum_{l=1}^{m} K^{l i}\left(t-\tau_{p}\right) \nu^{l p} \\
& \sum_{h=0}^{p-1} \sum_{l=1}^{m} E^{l i}\left(t-\tau_{h}\right) \Delta D_{n}^{l h}+\sum_{h=0}^{p-1} \sum_{l=1}^{m} F^{l i}\left(t-\tau_{h}\right) \Delta D_{s}^{l h}+\sum_{h=0}^{p-1} \sum_{l=1}^{m} K^{l i}\left(t-\tau_{h}\right) \nu^{l h} \\
& +\sum_{h=0}^{p} \sum_{l=1}^{m} S^{l i}\left(t-\tau_{h}\right) \nu_{h}^{l h} \\
& \Delta p^{i}(t)=\sum_{l=1}^{m} L^{l i}\left(t-\tau_{p}\right) \Delta D_{n}^{l p}+\sum_{l=1}^{m} M^{l i}\left(t-\tau_{p}\right) \Delta D_{s}^{l p}+\sum_{l=1}^{m} N^{l i}\left(t-\tau_{p}\right) \nu^{l p} \\
& \sum_{h=0}^{p-1} \sum_{l=1}^{m} L^{l i}\left(t-\tau_{h}\right) \Delta D_{n}^{l h}+\sum_{h=0}^{p-1} \sum_{l=1}^{m} M^{l i}\left(t-\tau_{h}\right) \Delta D_{s}^{l h}+\sum_{h=0}^{p-1} \sum_{l=1}^{m} N^{l i}\left(t-\tau_{h}\right) \nu^{l h} \\
& +\sum_{h=0}^{p} \sum_{l=1}^{m} P^{l i}\left(t-\tau_{h}\right) \nu_{h}^{l h}
\end{aligned}
$$

where $\Delta \mathrm{D}_{\mathrm{n}}^{\mathrm{lp}}$, and $\Delta \mathrm{D}_{\mathrm{s}}^{\mathrm{lp}}$ is $v^{\mathrm{lp}}$ are the increments of source strengths at time step $p$, which is current step; $\Delta \mathrm{D}_{\mathrm{n}}^{\mathrm{lh}}$, and $\Delta \mathrm{D}_{\mathrm{s}}^{\mathrm{lh}}$ is $v^{\text {lh }}$ are the previous increments of source strengths at time step $h$, which varies from 1 to p-1. $A^{l i}\left(t-\tau_{h}\right), B^{l i}\left(t-\tau_{h}\right), C^{l i}\left(t-\tau_{h}\right), E^{l i}\left(t-\tau_{h}\right), F^{l i}\left(t-\tau_{h}\right), K^{l i}(t-$ $\left.\tau_{h}\right), L^{l i}\left(t-\tau_{h}\right), M^{l i}\left(t-\tau_{h}\right), N^{l i}\left(t-\tau_{h}\right), R^{l i}\left(t-\tau_{h}\right), \mathrm{S}^{l i}\left(t-\tau_{h}\right), \mathrm{P}^{l i}\left(t-\tau_{h}\right)$, are the influence coefficients of $l^{\text {th }}$ fracture element on the $i^{\text {th }}$ fracture element at time step $h$. Using the same time discretizing, the effective stress change in $i^{\text {th }}$ fracture can be rewritten as:

$$
\begin{aligned}
\Delta \sigma_{n}^{i}(t)+p^{i}(t)-p_{0}^{i}= & -K_{n}\left(\Delta D_{n}^{i p}+\Delta D_{s}^{i p} \tan \phi_{d}\right) \\
& -K_{n}\left(\sum_{h=0}^{p-1} \Delta D_{n}^{i h}+\tan \phi_{d} \sum_{h=0}^{p-1} \Delta D_{s}^{i h}\right) \\
\Delta \sigma_{s}^{i}(t)= & K_{s} \Delta D_{s}^{i p}+K_{s} \sum_{h=0}^{p-1} \Delta D_{s}^{i h}
\end{aligned}
$$

where $p^{i}(t)$ is the fluid pressure in the $i^{t h}$ fracture element at time $t$ and $p_{0}^{i}$ is the initial fluid pressure in the $i^{\text {th }}$ fracture element. Substituting the last two Eqs. into Eqs.(49-51) and substituting $p^{i}(t)-p_{0}^{i}$ for $\Delta p^{i}(t)$ results:

$$
\begin{aligned}
& p^{i}(t)+\sum_{l=1}^{m} A^{l i}\left(t-\tau_{p}\right) \Delta D_{n}^{l p}+K_{n} \Delta D_{n}^{i p}+\sum_{l=1}^{m} B^{l i}\left(t-\tau_{p}\right) \Delta D_{s}^{l p}+K_{n} \tan \phi_{d} \Delta D_{s}^{i p} \\
& +\sum_{l=1}^{m} C^{l i}\left(t-\tau_{p}\right) \nu^{l p}=-\sum_{h=0}^{p-1} \sum_{l=1}^{m} A^{l i}\left(t-\tau_{h}\right) \Delta D_{n}^{l h}-\sum_{h=0}^{p-1} \sum_{l=1}^{m} B^{l i}\left(t-\tau_{h}\right) \Delta D_{s}^{l h} \\
& -\sum_{h=0}^{p-1} \sum_{l=1}^{m} C^{l i}\left(t-\tau_{h}\right) \nu^{l h}-\sum_{h=0}^{p} \sum_{l=1}^{m} R^{l i}\left(t-\tau_{h}\right) \nu_{h}^{l h}-K_{n}\left(\sum_{h=0}^{p-1} \Delta D_{n}^{i h}+\tan \phi_{d} \sum_{h=0}^{p-1} \Delta D_{s}^{i h}\right)+p_{0}^{i}
\end{aligned}
$$




$$
\begin{aligned}
& \sum_{l=1}^{m} E^{l i}\left(t-\tau_{p}\right) \Delta D_{n}^{l p}+\sum_{l=1}^{m} F^{l i}\left(t-\tau_{p}\right) \Delta D_{s}^{l p}-K_{s} \Delta D_{s}^{i p}+\sum_{l=1}^{m} K^{l i}\left(t-\tau_{p}\right) \nu^{l p}= \\
& -\sum_{h=0}^{p-1} \sum_{l=1}^{m} E^{l i}\left(t-\tau_{h}\right) \Delta D_{n}^{l h}-\sum_{h=0}^{p-1} \sum_{l=1}^{m} F^{l i}\left(t-\tau_{h}\right) \Delta D_{s}^{l h}-\sum_{h=0}^{p-1} \sum_{l=1}^{m} K^{l i}\left(t-\tau_{h}\right) \nu^{l h} \\
& -\sum_{h=0}^{p} \sum_{l=1}^{m} S^{l i}\left(t-\tau_{h}\right) v_{h}^{l h}+K_{s} \sum_{h=0}^{p-1} \Delta D_{s}^{i h} \\
& -p^{i}(t)+\sum_{l=1}^{m} L^{l i}\left(t-\tau_{p}\right) \Delta D_{n}^{l p}+\sum_{l=1}^{m} M^{l i}\left(t-\tau_{p}\right) \Delta D_{s}^{l p}+\sum_{l=1}^{m} N^{l i}\left(t-\tau_{p}\right) \nu^{l p}= \\
& -p_{0}^{i}-\sum_{h=0}^{p-1} \sum_{l=1}^{m} L^{l i}\left(t-\tau_{h}\right) \Delta D_{n}^{l h}-\sum_{h=0}^{p-1} \sum_{l=1}^{m} M^{l i}\left(t-\tau_{h}\right) \Delta D_{s}^{l h}-\sum_{h=0}^{p-1} \sum_{l=1}^{m} N^{l i}\left(t-\tau_{h}\right) v^{l h} \\
& -\sum_{h=0}^{p} \sum_{l=1}^{m} P^{l i}\left(t-\tau_{h}\right) v_{h}^{l h}
\end{aligned}
$$

The fluid flow equation (47) is discretized in space and time for a given fracture network.

$$
\sum_{j=1}^{m} C_{p}^{i j} p_{j}^{l}(t)-n \Delta L \Delta D_{n}^{i p}+\Delta L v^{i p}=n w_{f} \Delta L c_{f} p^{i}\left(\tau_{p}\right)-\Delta L \sum_{h=0}^{p-1} v^{i h}-\sum_{h=0}^{p} Q_{i}^{h}
$$

where $C_{p}{ }^{i j}$ is the fluid coefficient matrix. The production rate (or injection rate) from $\mathrm{i}^{\text {th }}$ fracture element $Q_{i}^{h}$ is also discretized in time in Eq. (57). All terms on the left side of Eqs (55)-(56) are unknown and all on the right side are known. When the production rate and initial reservoir pressure are specified, the normal and shear fracture displacement, interface flow rate, and fluid pressure can be obtained by solving the linear equation Eqs. (55)-(56). Unlike uncoupled methods, the interface flow rate (leak-off) is solved implicitly and it need not discretize the matrix to calculate interface flow rate using finite difference method. 


\section{References}

1. Palciauskas, V.V., and Domenico, P.A. 1982. Characterization of Drained and Undrained Response of Thermally Loaded Repository Rocks. Water Res. Res., 18, pp. 281-290.

2. McTigue, D.F. 1986. Thermoelastic Response of Fluid-saturated Porous Rock. J. Geophys. Res., 91, B9, pp. 9533-9542.

3. Coussy, O. 1991. Thermoporoelastic response of a borehole. Transport in Porous Media, 21, pp. 121-146.

4. Delaney, P.T. 1982. Rapid Intrusion of Magma into Wet Rock: Groundwater Flow Due to Pore Pressure Increases. J. Geophys Res., 87, B9, pp. 7739-7756.

5. Biot, M.A., 1941. General Theory of Three-dimensional Consolidation. J. Applied Phys., 12, pp. $155-164$

6. Detournay, E., and Cheng, A.H.-D. 1993. "Fundamentals of Poroelasticity", in J.A. Hudson (ed) Comprehensive Rock Engineering: Principles, Practices, and Projects, vol. 2, pp. 113 171.

7. Rice, J.R., and Cleary, M.P. 1976. Some Basic Stress-diffusion solutions for fluid saturated elastic media with compressible constituents. Reviews Geophys \& Space Phys, 14, pp. 227241.

8. Norris, A.N. 1992. On the Correspondence between Poroelasticity and Thermoelasticity. $J$. Applied Phys. 71, pp. 1138-1141.

9. Ghassemi, A., and Diek, A. 2002. Poro-thermoelasticity for Swelling Shales. J. Pet. Sci. \& Engrg, 34, pp. 123-135.

10. Wang, Y., and Papamichos, E. 1994. Conductive Heat Flow and Thermally Induced Fluid Flow around a Well Bore in a Poroelastic Medium. Water Res. Res., 30, 12, pp. 3375-3384.

11. Ghassemi, A., Cheng, A.H.-D., Diek, A., and Roegiers, J.-C., 2001. A Complete Plane- strain Fictitious Stress Boundary Element Method for Poroelastic Media. J. Engrg Anal. Boundary Elements, 25, 1, pp. 41-48.

12. Brebbia, C.A., Telles, J.C.F., and Wrobel, L.C. 1984. Boundary Element Techniques: Theory and Applications in Engineering, Springer-Verlag, Berlin, 464p

13. Crouch, S.L., and Starfield, A.M., 1983. Boundary Element Methods in Solid Mechanics. George Allen and Unwin, London, 322p

14. Cheng, A.H.-D., and Detournay, E., 1998. On Singular Integral Equations and Fundamental Solutions of Poroelasticity. Int. J. Solids Struct., 35, pp. 4521-4555.

15. Cheng, A.H.-D., 1998. On Generalized Plane Strain Poroelasticity. Int. J. Rock Mech. \& Min. Sci. 35, 2, pp. 183-193.

16. Ghassemi, A. \& Zhang, Q. 2004. A transient fictitious stress boundary element method for poro-thermoelastic media. J. Eng. Anal. Boundary Elements, 28/11, 1363-1373.

17. Berchenko, I., 1998. "Thermal Loading of Saturated Rock Mass: Field Experiment and Modeling Using Thermoporoelastic Singular Solutions", Ph.D. Dissertation, University of Minnesota

18. Cheng, A.H.-D., and Detournay, E. 1988. A Direct Boundary Element Method for Plane Strain Poroelasticity. Int. J. Num.Anal. Methods in Geomech., 12, pp. 551-572

19. Banerjee, P.K., Butterfield, R. 1981. Boundary element methods in engineering science, McGraw-Hill, London, 512p.

20. Curran, J.H., Carvalho, J.L. 1987. A Displacement Discontinuity Model for Fluid-saturated Porous Media", Proc. 6th Congress of the ISRM, 1, pp. 73-78.

21. Bandis S.C., Lumsden, A.C. and Barton, N.R.: Fundamentals of rock joint deformation. Int. J. Rock Mech. Min. Sci. and Geomech. Abstr. 20 (6), 249-268, 1983.

22. Cheng, A.-D., A. Ghassemi, and Detournay, E. 2001. A two-dimensional solution for heat extraction from a fracture in hot dry rock. Int. J. Numer. Analy. Meth. Geomech. 25: $1327-1338$. 


\section{Three-dimensional Boundary Element Method for Calculating Natural Fracture Response and Injection-Induced Stresses}

Thermally-induced stresses significantly contribute to seismicity in petroleum and geothermal fields [1, 2]. Reservoir seismicity and the variation of injectivity with water temperature in geothermal fields have been attributed to thermally-induced stresses. It has been found that half the earthquakes in The Geysers field seem to be associated with cold water injection [2]. The mechanism by which seismicity occurs is believed to be shear slip on natural fractures resulting from a reduction in effective stress acting across the fracture. The magnitude of the thermal stresses associated with advective cooling has been estimated analytically [3] using an axisymmetric model of injection into a planar reservoir and a 1D heat flow in the rock mass. It has been shown that one- and twodimensional heat flow models underestimate heat transfer to the fluid from the crack [4]. Thus, rock cooling and the associated thermal stresses should be studied using threedimensional heat transfer and stress models. This requires coupling a 3D heat flow model to a 3D elasticity model. A reason for ignoring the three-dimensional nature of heat conduction in the reservoir is the difficulty in treating the infinite geothermal reservoir geometry by numerical discretization. However, it has been demonstrated [4] that by using 3D Green's function for heat conduction and the integral equation formulation the need for discretizing the 3D reservoir is completely eliminated. In this work, we developed a three-dimensional numerical model to simulate the coupled poro-and thermoelastic processes related to cold water injection into an arbitrary shaped fracture in geothermal reservoirs. In the model, the fluid flow and the convective heat transfer in the fracture are modeled by the finite element method; while the three-dimensional pore fluid diffusion and heat conductive transfer in the reservoir matrix are modeled by the boundary integral equation method without discretizing the reservoir domain. The influence of the fracture aperture variation on the fluid flow in the fracture is also included in the model. We present a few numerical examples to examine the physical process of the fluid injection/extraction and facture slip.

\subsection{Governing Equations}

A schematic view of heat extraction from a fracture or a fracture zone in rock is illustrated in Figure 4.1. In this work, the fracture is assumed to be flat, of finite size, and with arbitrary shape. The geothermal reservoir, on the other hand, is of infinite extent. Other physical assumptions are similar to these postulated in [5]. Specifically, it is assumed that the geothermal reservoir is impermeable to water, has constant heat conduction properties, and is non-deformable. The heat storage and dispersion effects in the fracture fluid flow are negligible and production rate of hot water is equal to the injection rate in the fracture. It is further postulated that the fracture width is small such that the flow in the fracture is laminar and governed by the lubrication flow equation. 
The fracture apertures may vary during the fluid injection/extraction process. The reservoir is assumed to be poro-thermoelastic and its poro and thermoelastic properties are constant. The mathematical formulations for the problem are presented as below.

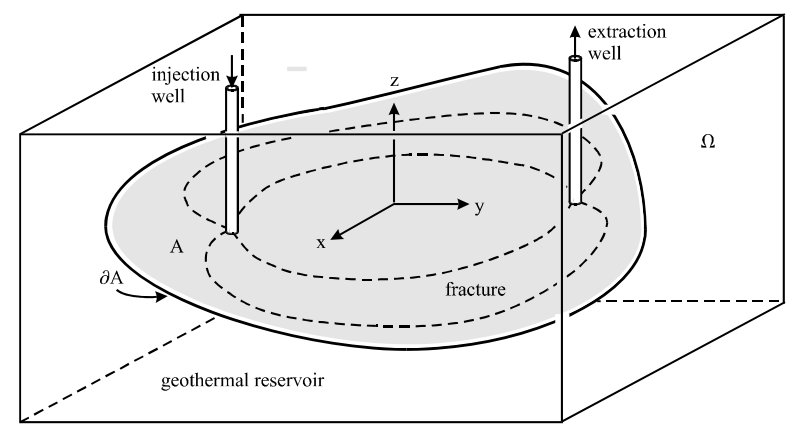

Figure 4.1. Cartoon of injection/extraction heat extraction in a planar fracture.

\subsubsection{Fluid flow in fracture}

The laminar incompressible fluid in the fracture is governed by the cubic law:

$$
\nabla_{2} p(x, y, 0, t)=-\frac{12 \mu}{w^{3}(x, y, t)} \mathbf{q}(x, y, t), \quad x, y \in A
$$

where $\nabla_{2}$ is the gradient operator in the fracture plane, $p(x, y, 0, t)$ the fluid pressure in the fracture, $\mu$ the fluid viscosity, $w(x, y, t)$ the fracture width, $\mathbf{q}(x, y, t)$ the fluid discharge, and $A$ is the fracture plane. It should be noted that $w(x, y, t)$ varies with the time.

For incompressible fluid and variable fracture aperture, we can write the fluid continuity equation as

$-\nabla_{2} \cdot \mathbf{q}(x, y, t)-2 v_{l}(x, y, t)+Q_{i}(t) \delta\left(x-x_{i}, y-y_{i}\right)-Q_{e}(t) \delta\left(x-x_{e}, y-y_{e}\right)=\frac{\partial w(x, y, t)}{\partial t}$

where $\nabla_{2}$. is the two-dimensional divergence operator, $v_{l}$ the fluid leak-off from one side of the fracture wall into reservoir matrix, $\partial w / \partial t$ is the rate of volume increase, $Q_{i}(t)$ and $Q_{e}(t)$ respectively the fluid injection and extraction rates, $\left(x_{i}, y_{i}\right)$ and $\left(x_{e}, y_{e}\right)$ respectively injection and extraction well locations, and $\delta$ the Dirac delta function.

Substitution of Eq. (1) into Eq. (2) yields the following governing equation for the fluid motion in the fracture

$$
\begin{aligned}
& \nabla_{2} \cdot\left[\frac{w^{3}(x, y, t)}{12 \mu} \nabla_{2} p(x, y, 0, t)\right]-2 v_{l}(x, y, t) \\
& =\frac{\partial w(x, y, t)}{\partial t}-Q_{i}(t) \delta\left(x-x_{i}, y-y_{i}\right)+Q_{e}(t) \delta\left(x-x_{e}, y-y_{e}\right)
\end{aligned}
$$




\subsubsection{Fluid flow in reservoir matrix}

As fluid is injected into the fracture or fracture zone, it will leak into rock matrix. This fluid loss can be expressed using Darcy's law:

$$
v_{l}(x, y, t)=-\left.\kappa \frac{\partial p(x, y, z, t)}{\partial n}\right|_{z=0}
$$

where $\kappa$ is the rock permeability, $n$ is the outward normal of the fracture surface, $z=0$ denotes the fracture surface.

The three-dimensional field equations for the poroelastic rock matrix can be presented as a Navier equation with a coupling term and a diffusion equation [6]:

$$
\begin{gathered}
G \nabla_{2}^{2} u_{i}+\frac{G}{1-2 v} u_{k, k i}-\alpha p_{, i}=0 \\
\frac{\partial p}{\partial t}-\frac{2 \kappa G B^{2}(1-2 v)\left(1+v_{u}\right)^{2}}{9\left(v_{u}-v\right)\left(1-2 v_{u}\right)} \nabla_{3}^{2} p=-\frac{2 G B\left(1+v_{u}\right)}{3\left(1-2 v_{u}\right)} \frac{\partial \varepsilon}{\partial t}
\end{gathered}
$$

where $u_{i}$ is the solid displacement in the $i$ direction, $p$ is the pore pressure, $G$ is the shear modulus, $v$ and $v_{u}$ respectively are the drained and undrained Poisson's ratios, $\alpha$ is the Biot's coefficient of effective stress, $B$ is the Skempton's pore pressure coefficient, $\varepsilon$ is the volumetric strain, and the other notations are the same as those defined previously. Note that the same notation $p$ is used for the pressure of reservoir matrix and water in the fracture, because the pressure must be continuous between the two subsystems (assuming no filter cake).

\subsubsection{Heat transport in fracture}

For the heat transport in the fracture, it has been demonstrated that under the conditions of relatively large advection velocity and continuous injection of fluid, the heat storage and diffusion are negligible compared to the heat advection by the fluid flow in the fracture [5,7]. Therefore, the heat transport in the fracture can be expressed as

$$
\rho_{w} C_{w} \mathbf{q}(x, y) \cdot \nabla_{2} T(x, y, 0, t)+q_{T}(x, y, 0, t)=0
$$

where $\rho_{w}$ is the fluid density, $c_{w}$ is the specific heat of fluid, $q_{T}$ is the heat source intensity which denotes the heat transfer rate between the reservoir matrix and the fluid in the fracture.

\subsubsection{Heat transport in reservoir}

The heat transport takes place both in the geothermal reservoir and the fracture. In the low permeability geothermal reservoir matrix, the heat conduction is the dominated way of heat transport and can be expressed as:

$$
K_{r} \nabla_{3}^{2} T(x, y, z, t)=\rho_{r} c_{r} \frac{\partial T(x, y, z, t)}{\partial t}, x, y, z \in \Omega
$$

where $T$ is rock temperature, $\rho_{r}$ is the rock density, $c_{r}$ is the specific heat of rock, $\nabla_{3}^{2}$ is the Laplacian operator in three dimensions, and $\Omega$ represents the geothermal reservoir. 
By assuming that the temperature is continuous across the fracture surface $(z=0)$, the heat source intensity in Eq. (7) can be expressed as

$q_{T}(x, y, 0, t)=-\left.2 K_{r} \frac{\partial T(x, y, z, t)}{\partial z}\right|_{z=0}$

where $K_{r}$ is the rock thermal conductivity.

Except the above governing equations, we have to set the boundary and initial conditions for the present problem. In this work, the injection rate $Q_{i}(t)$ or injection pressure $p_{i}(t)$, the extraction rate $Q_{i}(t)$ or extraction pressure $p_{e}(t)$, and the temperature of the injected fluid are given. We also assume the fracture circumference is fluid impermeable. Meanwhile, the initial fracture aperture, initial reservoir stress, pore pressure and temperature fields are also required. It should be noted that the temperature of the extracted fluid is unknown and a part of the solutions.

\subsection{Integral Equation Method}

The fracture in a poroelastic medium may be regarded as a surface across which the solid displacements, normal fluid flux, and normal heat flux are discontinuous. Such a discontinuity surface can mathematically be simulated by a distribution over time and space of impulse point displacement discontinuities (DD), fluid sources and heat sources. If the density of these singularities is known, integral representations of the field quantities, such as solid displacement, fluid flux, stresses, pore pressure and temperature, can be evaluated using the integral equation method [8]. In particular, the integral representations of the stresses, pore pressure, and temperature at any location in the reservoir can be respectively expressed as:

$$
\begin{aligned}
& \sigma_{i j}(\mathbf{x}, t)=\int_{0}^{t} \int_{A} \sigma_{i j k n}^{i d}\left(\mathbf{x}-\mathbf{x}^{\prime}, t-t^{\prime}\right) D_{k n}\left(\mathbf{x}^{\prime}, t^{\prime}\right) d A\left(\mathbf{x}^{\prime}\right) d t^{\prime}+\int_{0}^{t} \int_{A} \sigma_{i j}^{i f}\left(\mathbf{x}-\mathbf{x}^{\prime}, t-t^{\prime}\right) D_{f}\left(\mathbf{x}^{\prime}, t^{\prime}\right) d A\left(\mathbf{x}^{\prime}\right) d t^{\prime} \\
& +\int_{0}^{t} \int_{A} \sigma_{i j}^{i h}\left(\mathbf{x}-\mathbf{x}^{\prime}, t-t^{\prime}\right) D_{h}\left(\mathbf{x}^{\prime}, t^{\prime}\right) d A\left(\mathbf{x}^{\prime}\right) d t^{\prime}+\sigma_{i j 0}(\mathbf{x}) \\
& \quad p(\mathbf{x}, t)=\int_{0}^{t} \int_{A} p_{i j}^{i d}\left(\mathbf{x}-\mathbf{x}^{\prime}, t-t^{\prime}\right) D_{i j}\left(\mathbf{x}^{\prime}, t^{\prime}\right) d A\left(\mathbf{x}^{\prime}\right) d t^{\prime}+\int_{0}^{t} \int_{A} p^{i f}\left(\mathbf{x}-\mathbf{x}^{\prime}, t-t^{\prime}\right) D_{f}\left(\mathbf{x}^{\prime}, t^{\prime}\right) d A\left(\mathbf{x}^{\prime}\right) d t^{\prime} \\
& \quad+\int_{0}^{t} \int_{A} p^{i h}\left(\mathbf{x}-\mathbf{x}^{\prime}, t-t^{\prime}\right) D_{h}\left(\mathbf{x}^{\prime}, t^{\prime}\right) d A\left(\mathbf{x}^{\prime}\right) d t^{\prime}+p_{0}(\mathbf{x}) \\
& T(\mathbf{x}, t)=\int_{0}^{t} \int_{A} T^{i h}\left(\mathbf{x}-\mathbf{x}^{\prime}, t-t^{\prime}\right) D_{h}\left(\mathbf{x}^{\prime}, t^{\prime}\right) d A\left(\mathbf{x}^{\prime}\right) d t^{\prime}+T_{0}
\end{aligned}
$$

where $A$ denotes the fracture surface, $t$ denotes time, $D_{k n}$ (or $D_{i j}$ ), $D_{f}$ and $D_{h}$ are respectively the displacement discontinuity, the fluid source intensity, and the heat source intensity; $\sigma_{i j k n}^{i d}, \sigma_{i j}^{i f}, \sigma_{i j}^{i h}, p_{i j}^{i d}, p^{i f}$ and $p^{i h}$ are the instantaneous fundamental solutions, i.e. the incremental stresses, pore pressures and temperature due to a unit impulse of the

displacement discontinuity ("id"), the fluid source intensity ("if”) and the heat source intensity ("ih"); and $\sigma_{i j 0}, p_{0}$ and $T_{0}$ are respectively the initial stresses, pore pressure, and temperature. 
Application of Eqs. (10) (12) at the fracture yields equations for the densities of displacement discontinuities, fluid source intensity and heat source intensity, we obtain the expressions for the normal stresses, pore pressures and temperatures at the fracture surface as below:

$$
\begin{gathered}
\sigma_{n}(\mathbf{x}, t)=\int_{0}^{t} \int_{A} \sigma_{n n}^{i d}\left(\mathbf{x}-\mathbf{x}^{\prime}, t-t^{\prime}\right) D_{n}\left(\mathbf{x}^{\prime}, t^{\prime}\right) d A\left(\mathbf{x}^{\prime}\right) d t^{\prime}+ \\
\int_{0}^{t} \int_{A} \sigma_{n}^{i f}\left(\mathbf{x}-\mathbf{x}^{\prime}, t-t^{\prime}\right) D_{f}\left(\mathbf{x}^{\prime}, t^{\prime}\right) d A\left(\mathbf{x}^{\prime}\right) d t^{\prime}+\int_{0}^{t} \int_{A} \sigma_{n}^{i h}\left(\mathbf{x}-\mathbf{x}^{\prime}, t-t^{\prime}\right) D_{h}\left(\mathbf{x}^{\prime}, t^{\prime}\right) d A\left(\mathbf{x}^{\prime}\right) d t^{\prime}+\sigma_{n 0}(\mathbf{x}) \\
\sigma_{z x}(\mathbf{x}, t)=\int_{0}^{t} \int_{A}\left\{\sigma_{z x z x}^{i d}\left(\mathbf{x}-\mathbf{x}^{\prime}, t-t^{\prime}\right) D_{z x}\left(\mathbf{x}^{\prime}, t^{\prime}\right)+\sigma_{z x y y}^{i d}\left(\mathbf{x}-\mathbf{x}^{\prime}, t-t^{\prime}\right) D_{z y}\left(\mathbf{x}^{\prime}, t^{\prime}\right)\right\} d A\left(\mathbf{x}^{\prime}\right) d t^{\prime}+\sigma_{z x 0}(\mathbf{x}) \\
\sigma_{z y}(\mathbf{x}, t)=\int_{0}^{t} \int_{A}\left\{\sigma_{z y z x}^{i d}\left(\mathbf{x}-\mathbf{x}^{\prime}, t-t^{\prime}\right) D_{z x}\left(\mathbf{x}^{\prime}, t^{\prime}\right)+\sigma_{z y z y}^{i d}\left(\mathbf{x}-\mathbf{x}^{\prime}, t-t^{\prime}\right) D_{z y}\left(\mathbf{x}^{\prime}, t^{\prime}\right)\right\} d A\left(\mathbf{x}^{\prime}\right) d t^{\prime}+\sigma_{z y 0}(\mathbf{x}) \\
p(\mathbf{x}, t)=\int_{0}^{t} \int_{A} p_{n}^{i d}\left(\mathbf{x}-\mathbf{x}^{\prime}, t-t^{\prime}\right) D_{n}\left(\mathbf{x}^{\prime}, t^{\prime}\right) d A\left(\mathbf{x}^{\prime}\right) d t^{\prime}+ \\
\int_{0}^{t} \int_{A} p^{i f}\left(\mathbf{x}-\mathbf{x}^{\prime}, t-t^{\prime}\right) D_{f}\left(\mathbf{x}^{\prime}, t^{\prime}\right) d A\left(\mathbf{x}^{\prime}\right) d t^{\prime}+\int_{0}^{t} \int_{A} p^{i h}\left(\mathbf{x}-\mathbf{x}^{\prime}, t-t^{\prime}\right) D_{h}\left(\mathbf{x}^{\prime}, t^{\prime}\right) d A\left(\mathbf{x}^{\prime}\right) d t^{\prime}+p_{0}(\mathbf{x}) \\
T(\mathbf{x}, t)=\int_{0}^{t} \int_{A} T^{i h}\left(\mathbf{x}-\mathbf{x}^{\prime}, t-t^{\prime}\right) D_{h}\left(\mathbf{x}^{\prime}, t^{\prime}\right) d A\left(\mathbf{x}^{\prime}\right) d t^{\prime}+T_{0}(\mathbf{x})
\end{gathered}
$$

where the left hand side of Eq. (13) is the normal and shear stress components at the fracture surface, and the left hand sides of Eqs. (14) and (15) are equal to the fluid pressure and temperature in the fracture.

Based on the above discretization schemes in the spatial and temporal domains, we can write the integrations in Eqs. (13) and (14) as below:

$$
\begin{aligned}
& \int_{0}^{t} \int_{A} \sigma_{z z z z}^{i d}\left(\mathbf{x}-\mathbf{x}^{\prime}, t-t^{\prime}\right) D_{z z}\left(\mathbf{x}^{\prime}, t^{\prime}\right) d A\left(\mathbf{x}^{\prime}\right) d t^{\prime} \\
& =\left[\sum_{m=1}^{M} \int_{A_{m}} \sigma_{z z z z(1)}^{c d}\left(\mathbf{x}-\mathbf{x}^{\prime}\right) d A\left(\mathbf{x}^{\prime}\right)\right] \mathbf{D}_{z z}(N \Delta t)+\sum_{n=1}^{N-1}\left[\sum_{m=1}^{M} \int_{A_{m}} \sigma_{z z z z(N-n+1)}^{c d}\left(\mathbf{x}-\mathbf{x}^{\prime}\right) d A\left(\mathbf{x}^{\prime}\right)\right] \mathbf{D}_{z z}(n \Delta t) \\
& \int_{0}^{t} \int_{A} \sigma_{z z}^{i s}\left(\mathbf{x}-\mathbf{x}^{\prime}, t-t^{\prime}\right) D_{f}\left(\mathbf{x}^{\prime}, t^{\prime}\right) d A\left(\mathbf{x}^{\prime}\right) d t^{\prime} \\
& =\left[\sum_{m=1}^{M} \int_{A_{m}} \mathbf{N}^{(m)} \sigma_{z z(1)}^{c s}\left(\mathbf{x}-\mathbf{x}^{\prime}\right) d A\left(\mathbf{x}^{\prime}\right)\right] \mathbf{D}_{f}(N \Delta t)+\sum_{n=1}^{N-1}\left[\sum_{m=1}^{M} \int_{A_{m}} \mathbf{N}^{(m)} \sigma_{z z(N-n+1)}^{c s}\left(\mathbf{x}-\mathbf{x}^{\prime}\right) d A\left(\mathbf{x}^{\prime}\right)\right] \mathbf{D}_{f}(n \Delta t) \\
& \int_{0}^{t} \int_{A} p_{z z}^{i d}\left(\mathbf{x}-\mathbf{x}^{\prime}, t-t^{\prime}\right) D_{z z}\left(\mathbf{x}^{\prime}, t^{\prime}\right) d A\left(\mathbf{x}^{\prime}\right) d t^{\prime} \\
& =\left[\sum_{m=1}^{M} \int_{A_{m}} p_{z z(1)}^{c d}\left(\mathbf{x}-\mathbf{x}^{\prime}\right) d A\left(\mathbf{x}^{\prime}\right)\right] \mathbf{D}_{z z}(N \Delta t)+\sum_{n=1}^{N-1}\left[\sum_{m=1}^{M} \int_{A_{m}} p_{z z(N-n+1)}^{c d}\left(\mathbf{x}-\mathbf{x}^{\prime}\right) d A\left(\mathbf{x}^{\prime}\right)\right] \mathbf{D}_{z z}(n \Delta t)
\end{aligned}
$$




$$
\begin{aligned}
& \int_{0}^{t} \int_{A} p^{i s}\left(\mathbf{x}-\mathbf{x}^{\prime}, t-t^{\prime}\right) D_{f}\left(\mathbf{x}^{\prime}, t^{\prime}\right) d A\left(\mathbf{x}^{\prime}\right) d t^{\prime} \\
& =\left[\sum_{m=1}^{M} \int_{A_{m}} \mathbf{N}^{(m)} p_{(1)}^{c s}\left(\mathbf{x}-\mathbf{x}^{\prime}\right) d A\left(\mathbf{x}^{\prime}\right)\right] \mathbf{D}_{f}(N \Delta t)+\sum_{n=1}^{N-1}\left[\sum_{m=1}^{M} \int_{A_{m}} \mathbf{N}^{(m)} p_{(N-n+1)}^{c s}\left(\mathbf{x}-\mathbf{x}^{\prime}\right) d A\left(\mathbf{x}^{\prime}\right)\right] \mathbf{D}_{f}(n \Delta t)
\end{aligned}
$$

where $M$ and $N$ are respectively element numbers and the current time step, $\mathbf{N}^{(m)}$ is shape functions for element $m, \mathbf{D}_{z z}(N \Delta t)$ is the unknown normal DD vector and $\mathbf{D}_{f}(N \Delta t)$ is the unknown fluid source intensity vector, the superscripts $c d$ and $c s$ denote the fundamental solutions due to the continuous unit DDs and fluid source, respectively. Because the fundamental solution due to an instantaneous singularity is the time derivative of the fundamental solution due to a continuous singularity, the integrals of instantaneous fundamental solutions in the time domain can be conveniently expressed using their corresponding continuous fundamental solutions to develop the convolution time integrals that are then evaluated by going back to zero at every time step. In this way, domain integration is circumvented. In the above equations, the following definitions are also used:

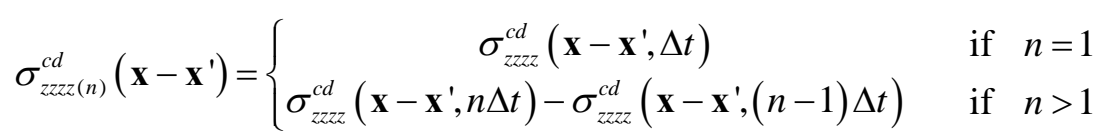

and $\sigma_{z 11}^{c s}, \sigma_{z z(n)}^{c s}, p_{z 11}^{c d}, p_{z z(n)}^{c d}, p_{1}^{c s}$ and $p_{n}^{c s}$ are defined in the same way. It should be noted that all the second terms on the right hand side of Eqs. (16)-(19) can be evaluated directly because only the known DDs and fluid source intensities from the previous time steps are involved. Substituting Eqs. (16)-(19) into Eqs. (13) and (14), yields:

$$
\begin{gathered}
\sigma_{z z}(\mathbf{x}, t)-\sigma_{z z 0}(\mathbf{x})=\left[\sum_{m=1}^{M} \int_{A_{m}} \sigma_{z z z z 1}^{c d}\left(x-x^{\prime}, y-y^{\prime}, 0\right) d A\left(\mathbf{x}^{\prime}\right)\right] \mathbf{D}_{z z}(N \Delta t) \\
+\left[\sum_{m=1}^{M} \int_{A_{m}} \mathbf{N}^{(m)} \sigma_{z z 1}^{c s}\left(\mathbf{x}-\mathbf{x}^{\prime}\right) d A\left(\mathbf{x}^{\prime}\right)\right] \mathbf{D}_{f}(N \Delta t)+\sigma_{z z 1}(\mathbf{x}, t) \\
p(\mathbf{x}, t)-p_{0}(\mathbf{x})=\left[\sum_{m=1}^{M} \int_{A_{m}} p_{z z}^{c d}\left(\mathbf{x}-\mathbf{x}^{\prime}, 0\right) d A\left(\mathbf{x}^{\prime}\right)\right] \mathbf{D}_{z z}(N \Delta t) \\
+\left[\sum_{m=1}^{M} \int_{A_{m}} \mathbf{N}^{(m)} p^{c s}\left(\mathbf{x}-\mathbf{x}^{\prime}\right) d A\left(\mathbf{x}^{\prime}\right)\right] \mathbf{D}_{f}(N \Delta t)+p_{1}(\mathbf{x}, t)
\end{gathered}
$$




$$
\begin{aligned}
& \sigma_{z x}(\mathbf{x}, t)-\sigma_{z x 0}(\mathbf{x})=\left[\sum_{m=1}^{M} \int_{A_{m}} \sigma_{z x z x 1}^{c d}\left(x-x^{\prime}, y-y^{\prime}, 0\right) d A\left(\mathbf{x}^{\prime}\right)\right] \mathbf{D}_{z x}(N \Delta t) \\
& +\left[\sum_{m=1}^{M} \int_{A_{m}} \sigma_{z x z y 1}^{c d}\left(x-x^{\prime}, y-y^{\prime}, 0\right) d A\left(\mathbf{x}^{\prime}\right)\right] \mathbf{D}_{z y}(N \Delta t)+\sigma_{z x 1}(\mathbf{x}, t) \\
& \sigma_{z y}(\mathbf{x}, t)-\sigma_{z y 0}(\mathbf{x})=\left[\sum_{m=1}^{M} \int_{A_{m}} \sigma_{z y z x 1}^{c d}\left(x-x^{\prime}, y-y^{\prime}, 0\right) d A\left(\mathbf{x}^{\prime}\right)\right] \mathbf{D}_{z x}(N \Delta t) \\
& +\left[\sum_{m=1}^{M} \int_{A_{m}} \sigma_{z y z y 1}^{c d}\left(x-x^{\prime}, y-y^{\prime}, 0\right) d A\left(\mathbf{x}^{\prime}\right)\right] \mathbf{D}_{z y}(N \Delta t)+\sigma_{z y 1}(\mathbf{x}, t)
\end{aligned}
$$

in which:

$$
\begin{gathered}
\sigma_{z x 1}(\mathbf{x}, t)=\sum_{n=1}^{N-1}\left[\sum_{m=1}^{M} \int_{A_{m}} \sigma_{z x z x(N-n+1)}^{c d}\left(\mathbf{x}-\mathbf{x}^{\prime}\right) d A\left(\mathbf{x}^{\prime}\right)\right] \mathbf{D}_{z x}(n \Delta t) \\
+\sum_{n=1}^{N-1}\left[\sum_{m=1}^{M} \int_{A_{m}} \sigma_{z x z y(N-n+1)}^{c d}\left(\mathbf{x}-\mathbf{x}^{\prime}\right) d A\left(\mathbf{x}^{\prime}\right)\right] \mathbf{D}_{z y}(n \Delta t) \\
\sigma_{z y 1}(\mathbf{x}, t)=\sum_{n=1}^{N-1}\left[\sum_{m=1}^{M} \int_{A_{m}} \sigma_{z y z x(N-n+1)}^{c d}\left(\mathbf{x}-\mathbf{x}^{\prime}\right) d A\left(\mathbf{x}^{\prime}\right)\right] \mathbf{D}_{z x}(n \Delta t) \\
+\sum_{n=1}^{N-1}\left[\sum_{m=1}^{M} \int_{A_{m}} \sigma_{z y z y(N-n+1)}^{c d}\left(\mathbf{x}-\mathbf{x}^{\prime}\right) d A\left(\mathbf{x}^{\prime}\right)\right] \mathbf{D}_{z y}(n \Delta t)
\end{gathered}
$$

\subsection{Treatment of Joint Deformation}

The integral equations presented in the previous section can be used to study both hydraulic fractures and joints. A hydraulic fracture usually is pressurized in excess of the minimum in-situ stress and remains open during the loading process, which means both the normal and shear stiffness of the fracture are zero. On the hydraulic fracture surface, the normal tractions equal the fluid pressures in the fracture and the shear tractions equal zero. However, a different approach must be used for joints, as the joint normal and shear stiffness are nonzero and the normal and shear tractions on a joint change with joint normal and shear displacements. The required procedure for modeling the joint element is similar to that used by Ghassemi et al. [9], however, the fracture is discretized into a number of four-nodded quadrilateral elements in this work. During the fracture pressurization, each element can be either in a state of "separation", "stick" or "slip"; the element is closed in the latter two states. For any element, its state may change from one time step to another time step and must be determined at every time step because the computational methods for the terms on the left hand sides of Eqs. (21)-(23) are different for different element states as shown below. We denote the normal stiffness of the fracture as $K_{n}$. The shear stiffness of the fracture may be different in different shear directions. However, here we assume the shear stiffnesses are the same in all shear directions for simplification and denote it as $K_{s}$. The fracture aperture increment for any closed element " $i$ ” can be expressed as:

$$
\Delta \stackrel{i}{d}_{n}=\stackrel{i}{D}_{z z}+i_{d i l}^{i}=\left(\begin{array}{c}
i \\
\frac{-\Delta \sigma_{n}^{\prime}}{i} \\
\stackrel{K}{n}_{n}
\end{array}\right)+\stackrel{i}{a}_{d i l}
$$


where $\Delta \sigma_{n}^{i}$ is the increment of the normal effective stress and $\stackrel{i}{a}_{\text {dil }}$ is the dilation-induced aperture increase due to shear slip. In this work, for simplification, we assume the shear dilation is the same in all shear slip directions. As a result, the shear dilation for any element " $i$ " may be simply calculated by the following relation:

$$
\stackrel{i}{a_{d i l}}=\sqrt{\stackrel{i}{D_{z x}^{2}+D_{z y}^{2}}} \tan (\stackrel{i}{\phi} \text { dil })
$$

where $\stackrel{i}{D_{z x}}$ and $\stackrel{i}{D_{z y}}$ are the shear displacement components in $x$ and $y$ direction, respectively and $\stackrel{i}{\phi}_{d i}$ is the fracture dilation angle.

The problem is solved by marching in time. For any time step, $k$, we assume an initial values for the aperture change vector $a_{d i l}^{(k)}$ for all of the elements. In this work, we assume $\boldsymbol{a}_{d i l}^{(k)}=\mathbf{0}$ when $k=1$ and $\boldsymbol{a}_{d i l}^{(k)}=\boldsymbol{a}_{d i l}^{(k-1)}$ when $k>1$. Firstly, we do the computations in the normal direction using Eqs. For normal stress and pore pressure on the fracture. If any element $i$ is closed, its corresponding component on left hand side of Eqn for normal stress, the increment of the normal stress, can be written as $-\stackrel{i}{K_{n}}\left(\Delta \stackrel{i}{d_{n}}-\stackrel{i}{a}_{d i l}\right)+\Delta p$, where the first part is the normal effective stress increment which is obtained from Eq. (26) while the second part is the pore pressure increment. If any element $i$ is in "separation" state, its normal traction is the current fluid pressure, $p$, and therefore, the corresponding component on the left hand side of the equation for normal stress is $p-\stackrel{i}{\sigma_{z z 0}}$, where $\stackrel{i}{\sigma z z}$ is the initial normal stress at the center of element $i$.

At the start of the solution process, we assume that all fracture elements are closed and solve the linear equation system of equations for normal stress and pore pressure. Then, we calculate the resultant normal effective stress $\stackrel{i}{\sigma_{n}^{\prime}}$ which equals $-\stackrel{i}{K_{n}}\left(\begin{array}{c}i \\ \Delta d_{n}-\stackrel{i}{a_{d i l}}\end{array}\right)+\stackrel{i}{\sigma^{\prime}}{ }_{z z 0}$, where $\sigma^{i}{ }_{z z 0}$ is the initial normal effective stress. As shown in Figure 4.2, the following element separation criterion is adopted to judge whether the element $i$ is closed or in “separation” state:

$$
\stackrel{i}{\sigma_{n}^{\prime}}=\stackrel{i}{c} \stackrel{i}{c} \operatorname{cotan} \stackrel{i}{\phi}_{\text {eff }}
$$

where $\stackrel{i}{c}$ is the cohesion and $\stackrel{i}{\phi}_{\text {eff }}$ is the effective friction angle of the fracture surface.

Each element's predicted status in the normal direction is used for the next iterative step in the normal direction. The computation iteration in the normal direction stops when all the elements have the same status in the current iteration as in the last iteration. 


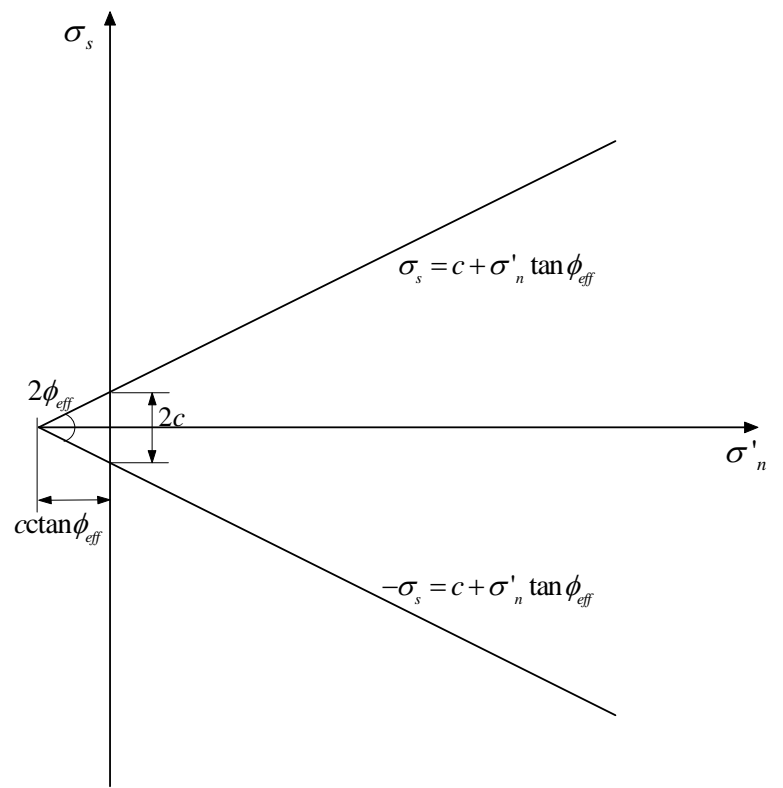

Figure 4.2. Mohr-Coulomb model used for joint elements.

After obtaining the element states in the normal direction and the magnitudes of the normal effective stresses, we use the following method to determine whether a closed element is in "stick" or "slip" state in the shear direction and calculate the shear displacements. For any element $i$ with the state of "separation" in the normal direction, its current shear tractions equal zero so that its corresponding components on the left hand sides of Eqs. (22) and (23) become $-i_{z x 0}$ and $-i_{z y 0}$, respectively. However, if the element $i$ is closed in the normal direction, we use Mohr-Coulomb model (Figure 4.2) to judge whether its state in the maximum allowable shear direction is "stick" or "slip". For a typical element, $i$, such a constraint can be expressed as:

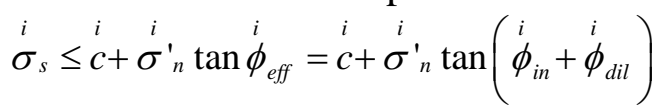

where $\stackrel{i}{\sigma}_{s}$ is the shear stress, $\stackrel{i}{\phi}_{\text {in }}$ the intrinsic friction angle, $\stackrel{i}{\phi}_{\text {dil }}$ the dilation angle, and the other notations have been defined before.

If element $i$ is in the "stick" status, the fracture deformation in the shear direction is assumed to deform in a linear elastic manner with its shear stiffness denoted by $K_{s}$. As a result, the left hand sides of Eqs. (22) and (23) for element $i$ can be written as ${\stackrel{i}{\sigma_{z x}}}^{(k-1)}-\stackrel{i}{K_{s}}\left(\begin{array}{l}\stackrel{i}{(k)}^{(k)}-i_{z x}^{(k-1)} \\ D_{z x}\end{array}\right)$ and $\sigma_{z y}^{i(k-1)}-\stackrel{i}{K}_{s}\left(\begin{array}{c}i^{(k)} \\ D_{z y}-i_{z y}^{(k-1)}\end{array}\right)$, respectively; where for each expression, the first part is the shear traction in the last time step and the second part is caused by the fracture elastic shear deformation in the current time step. However, if element $i$ is in the state of "slip", the shear stress cannot exceed the value of the yield stress given in Eq. (29) and therefore, the left hand sides of Eqs. (22) and (23) for element 
$i$ become $\sigma_{s}^{*(k)} \cos \theta-\sigma_{z x 0}$ and $\sigma_{s}^{*(k)} \sin \theta-\sigma_{z y 0}$, respectively, where $*$ denotes the maximum shear stresses which is calculated by Eq. (29) and $\theta$ is the angle between the maximum shear stress direction and the $x$-axis.

For any time step $k$, firstly we assume all the elements are in the state of "stick" and solve the linear equation system of Eqs. (17) and (18). Then we use Eq. (29) for all the closed elements to judge whether their state is "stick" or "slip". Similar to the process in the normal direction, we use an iterative method to solve Eqs. (22) and (23) until the states of all the elements in the shear direction in the current iteration do not change compared to those in the last iteration.

After the calculations of $\mathbf{D}_{z z}$ and $\mathbf{p}_{f}$ in the normal direction, and $\mathbf{D}_{z x}$ and $\mathbf{D}_{z y}$ in the shear direction, we can obtain a new fracture shear dilation vector $\boldsymbol{C}_{d i l}^{(k)}$ by substituting the calculated shear displacements into Eq. (27). Using this new $\boldsymbol{A}_{d i l}^{(k)}$, we repeat the above procedure to calculate $\mathbf{D}_{z z}, \mathbf{p}_{f}, \mathbf{D}_{z x}$ and $\mathbf{D}_{z y}$ until the following condition is satisfied:

$$
\| \text { new } \quad a_{d i l}^{(k)} \text {-old } a_{\text {dil }}^{(k)}\|/\| \text { old } \quad a_{d i l}^{(k)} \|<\text { TOL }
$$

where TOL is a prescribed tolerance. Thereafter, the next time step begins.

Once the DDs and fluid, and heat source intensities are obtained through the method described above, Eqs. (10) and (11) can be used to calculate the stresses and pore pressures at any place in the rock matrix utilizing the same boundary integral method we presented previously. The resultant stress and pore pressure fields in the reservoir matrix are essential for analysis of the formation failure around the fracture.

\subsection{Finite Element Method for Fluid Flow and Heat Transport in Fracture}

We use the finite element method to model the fluid motion and heat transport in the fracture. The fracture plane is discretized into a number of four-noded quadrilateral elements and we assume the following interpolations for any element $m$ :

$$
p^{(m)}=\mathbf{N}^{(m)} \tilde{\mathbf{p}}, D_{f}^{(m)}=\mathbf{N}^{(m)} \tilde{\mathbf{D}}_{f}, T^{(m)}=\mathbf{N}^{(m)} \tilde{\mathbf{T}}, D_{h}^{(m)}=\mathbf{N}^{(m)} \tilde{\mathbf{D}}_{h}
$$

where the superscript $m$ denotes the element $m, \mathbf{N}^{(m)}$ are the interpolative functions, and $\tilde{\mathbf{p}}, \tilde{\mathbf{D}}_{f}, \tilde{\mathbf{T}}$ and $\tilde{\mathbf{D}}_{h}$ are the vectors of nodal fluid pressure, fluid source intensity, temperature and heat source intensity, respectively. 
The finite element formulas for the fluid flow and heat transport are as follows:

$$
\begin{aligned}
& \mathbf{A}_{1} \tilde{\mathbf{p}}(t)+\mathbf{A}_{2} \tilde{\mathbf{D}}_{f}(t)=\mathbf{B}_{1}(t) \\
& \mathbf{A}_{3} \tilde{\mathbf{T}}(t)+\mathbf{A}_{4} \tilde{\mathbf{D}}_{h}(t)=\mathbf{0}
\end{aligned}
$$

where

$$
\begin{gathered}
\mathbf{A}_{1}=\sum_{m=1}^{M} \int_{A_{e}} \frac{w^{3}}{12 \mu} \nabla^{T} \mathbf{N}^{(m)} \nabla \mathbf{N}^{(m)} d A \\
\mathbf{A}_{2}=\sum_{m=1}^{M} \int_{A_{e}} \mathbf{N}^{(m) T} \mathbf{N}^{(m)} d A \\
\mathbf{B}_{1}=-\sum_{m=1}^{M} \int_{A_{e}} \frac{\partial w}{\partial t} \mathbf{N}^{(m) T} d A+\left.\sum \mathbf{N}^{(i) T}\right|_{\left(x_{i}, y_{i}\right)} Q_{i}(t)-\left.\sum \mathbf{N}^{(e) T}\right|_{\left(x_{e}, y_{e}\right)} Q_{e}(t) \\
\mathbf{A}_{3}=\rho_{w} c_{w} \sum_{m=1}^{M} \int_{A_{e}}\left(\mathbf{N}^{(m)}+\overline{\mathbf{N}}^{(m)}\right) \mathbf{q}(x, y) \nabla \mathbf{N}^{(m)} d A \\
\mathbf{A}_{4}=\sum_{m=1}^{M} \int_{A_{e}} \mathbf{N}^{(m) T} \mathbf{N}^{(m)} d A \\
\overline{\mathbf{N}}^{(m)}=\frac{\bar{k} q_{j} \mathbf{N}_{j}^{(m)}}{q_{i} q_{i}}, i=x, y ; j=x, y
\end{gathered}
$$

where $M$ is the total number of the elements on the fracture plane, $\left.\mathbf{N}^{(i) T}\right|_{\left(x_{i}, y_{i}\right)}$ denote the shape functions at the fluid injection well which is located at $\left(x_{i}, y_{i}\right)$ within element $i$, and $\left.\mathbf{N}^{(e) T}\right|_{\left(x_{e}, y_{e}\right)}$ denote the shape functions at the fluid extraction point which is located at $\left(x_{e}, y_{e}\right)$ within element $e$. It should be noted that here we adopted the streamline upwind/Petrov-Galerkin (SUPG) finite element technique proposed by Brooks and Hughes [10] for the convective-dominated heat transport problems because the numerical stability is unavoidable when the conventional Galerkin finite element method is used. The expressions of the SUPG parameters $\bar{k}, q_{x}$ and $q_{y}$ in Eq. (39) can be found in [10].

We denote the fracture aperture as $\mathbf{w}$ which is spatial and temporal dependent. $\mathbf{w}$ influences $\tilde{\mathbf{p}}$ and $\tilde{\mathbf{D}}_{f}$ as well as $\tilde{\mathbf{T}}$ and $\tilde{\mathbf{D}}_{h}$ significantly; inversely, the reaction of the reservoir matrix due to $\tilde{\mathbf{p}}, \tilde{\mathbf{D}}_{f}$ and $\tilde{\mathbf{D}}_{h}$ also affect the magnitudes of $\mathbf{w}$. The iterative method used to solve this coupling will be explained in the next Sections.

\subsection{Discretization of Integral Equations for Response of Reservoir Matrix}

The integral equations (13)-(15) are discretized in the spatial domain by using the same mesh in the finite element method and in the temporal domain by using the convolution algorithm [11]. Here we use constant quadrilateral elements for DDs and linear quadrilateral elements for $D_{f}$ and $D_{h}$ as the same as those used in the finite element methods in Section 4.4. We also assume that DDs, $D_{f}$ and $D_{h}$ are constant over each time step. As a result, when $t=N \Delta t$, we have: 


$$
\begin{aligned}
& \int_{0}^{t} \int_{A} \sigma_{n n}^{i d}\left(\mathbf{x}-\mathbf{x}^{\prime}, t-t^{\prime}\right) D_{n}\left(\mathbf{x}^{\prime}, t^{\prime}\right) d A\left(\mathbf{x}^{\prime}\right) d t^{\prime} \\
& \simeq\left[\sum_{m=1}^{M} \int_{A_{e}} \sigma_{n n(1)}^{c d}\left(\mathbf{x}-\mathbf{x}^{\prime}\right) d A\left(\mathbf{x}^{\prime}\right)\right] \tilde{\mathbf{D}}_{n}(N \Delta t)+\sum_{k=1}^{N-1}\left[\sum_{m=1}^{M} \int_{A_{e}} \sigma_{n n(N-n+1)}^{c d}\left(\mathbf{x}-\mathbf{x}^{\prime}\right) d A\left(\mathbf{x}^{\prime}\right)\right] \tilde{\mathbf{D}}_{n}(k \Delta t) \\
& \int_{0}^{t} \int_{A} \sigma_{n}^{i f}\left(\mathbf{x}-\mathbf{x}^{\prime}, t-t^{\prime}\right) D_{f}\left(\mathbf{x}^{\prime}, t^{\prime}\right) d A\left(\mathbf{x}^{\prime}\right) d t^{\prime} \\
& \simeq \simeq\left[\sum_{m=1}^{M} \int_{A_{e}} \sigma_{n(1)}^{c f}\left(\mathbf{x}-\mathbf{x}^{\prime}\right) d A\left(\mathbf{x}^{\prime}\right)\right] \tilde{\mathbf{D}}_{f}(N \Delta t)+\sum_{k=1}^{N-1}\left[\sum_{m=1}^{M} \int_{A_{e}} \sigma_{n(N-n+1)}^{c f}\left(\mathbf{x}-\mathbf{x}^{\prime}\right) d A\left(\mathbf{x}^{\prime}\right)\right] \tilde{\mathbf{D}}_{f}(k \Delta t) \\
& \int_{0}^{t} \int_{A} \sigma_{n}^{i h}\left(\mathbf{x}-\mathbf{x}^{\prime}, t-t^{\prime}\right) D_{h}\left(\mathbf{x}^{\prime}, t^{\prime}\right) d A\left(\mathbf{x}^{\prime}\right) d t^{\prime} \\
& \simeq\left[\sum_{m=1}^{M} \int_{A_{e}} \sigma_{n(1)}^{c h}\left(\mathbf{x}-\mathbf{x}^{\prime}\right) d A\left(\mathbf{x}^{\prime}\right)\right] \tilde{\mathbf{D}}_{h}(N \Delta t)+\sum_{k=1}^{N-1}\left[\sum_{m=1}^{M} \int_{A_{e}} \sigma_{n(N-n+1)}^{c h}\left(\mathbf{x}-\mathbf{x}^{\prime}\right) d A\left(\mathbf{x}^{\prime}\right)\right] \tilde{\mathbf{D}}_{h}(k \Delta t) \\
& \int_{0}^{t} \int_{A} p_{n}^{i d}\left(\mathbf{x}-\mathbf{x}^{\prime}, t-t^{\prime}\right) D_{n}\left(\mathbf{x}^{\prime}, t^{\prime}\right) d A\left(\mathbf{x}^{\prime}\right) d t^{\prime} \\
& \simeq\left[\sum_{m=1}^{M} \int_{A_{e}} p_{n(1)}^{c d}\left(\mathbf{x}-\mathbf{x}^{\prime}\right) d A\left(\mathbf{x}^{\prime}\right)\right] \tilde{\mathbf{D}}_{n}(N \Delta t)+\sum_{k=1}^{N-1}\left[\sum_{m=1}^{M} \int_{A_{e}} p_{n(N-n+1)}^{c d}\left(\mathbf{x}-\mathbf{x}^{\prime}\right) d A\left(\mathbf{x}^{\prime}\right)\right] \tilde{\mathbf{D}}_{n}(k \Delta t) \\
& \int_{0}^{t} \int_{A} p^{i f}\left(\mathbf{x}-\mathbf{x}^{\prime}, t-t^{\prime}\right) D_{f}\left(\mathbf{x}^{\prime}, t^{\prime}\right) d A\left(\mathbf{x}^{\prime}\right) d t^{\prime} \\
& \simeq\left[\sum_{m=1}^{M} \int_{A_{e}} p_{(1)}^{c f}\left(\mathbf{x}-\mathbf{x}^{\prime}\right) d A\left(\mathbf{x}^{\prime}\right)\right] \tilde{\mathbf{D}}_{f}(N \Delta t)+\sum_{k=1}^{N-1}\left[\sum_{m=1}^{M} \int_{A_{e}} p_{(N-n+1)}^{c f}\left(\mathbf{x}-\mathbf{x}^{\prime}\right) d A\left(\mathbf{x}^{\prime}\right)\right] \tilde{\mathbf{D}}_{f}(k \Delta t) \\
& \int_{0}^{t} \int_{A} p^{i h}\left(\mathbf{x}-\mathbf{x}^{\prime}, t-t^{\prime}\right) D_{h}\left(\mathbf{x}^{\prime}, t^{\prime}\right) d A\left(\mathbf{x}^{\prime}\right) d t^{\prime} \\
& \simeq\left[\sum_{m=1}^{M} \int_{A_{e}} p_{(1)}^{c h}\left(\mathbf{x}-\mathbf{x}^{\prime}\right) d A\left(\mathbf{x}^{\prime}\right)\right] \tilde{\mathbf{D}}_{h}(N \Delta t)+\sum_{k=1}^{N-1}\left[\sum_{m=1}^{M} \int_{A_{e}} p_{(N-n+1)}^{c h}\left(\mathbf{x}-\mathbf{x}^{\prime}\right) d A\left(\mathbf{x}^{\prime}\right)\right] \tilde{\mathbf{D}}_{h}(k \Delta t) \\
& \int_{0}^{t} \int_{A} T^{i h}\left(\mathbf{x}-\mathbf{x}^{\prime}, t-t^{\prime}\right) D_{h}\left(\mathbf{x}^{\prime}, t^{\prime}\right) d A\left(\mathbf{x}^{\prime}\right) d t^{\prime} \\
& \simeq\left[\sum_{m=1}^{M} \int_{A_{e}} T_{(1)}^{c h}\left(\mathbf{x}-\mathbf{x}^{\prime}\right) d A\left(\mathbf{x}^{\prime}\right)\right] \tilde{\mathbf{D}}_{h}(N \Delta t)+\sum_{k=1}^{N-1}\left[\sum_{m=1}^{M} \int_{A_{e}} T_{(N-n+1)}^{c h}\left(\mathbf{x}-\mathbf{x}^{\prime}\right) d A\left(\mathbf{x}^{\prime}\right)\right] \tilde{\mathbf{D}}_{h}(k \Delta t)
\end{aligned}
$$

where $M$ and $N$ are respectively element numbers and the current time step, $\mathbf{N}^{(m)}$ is shape functions for element $m, \tilde{\mathbf{D}}_{n}(N \Delta t), \tilde{\mathbf{D}}_{f}(N \Delta t)$ and $\tilde{\mathbf{D}}_{h}(N \Delta t)$ are unknown vectors, $\tilde{\mathbf{D}}_{n}(N \Delta t), \tilde{\mathbf{D}}_{f}(N \Delta t)$ and $\tilde{\mathbf{D}}_{h}(N \Delta t)$ are unknown vectors, $\tilde{\mathbf{D}}_{n}(n \Delta t), \tilde{\mathbf{D}}_{f}(n \Delta t)$ and $\tilde{\mathbf{D}}_{h}(n \Delta t)(\mathrm{n}<\mathrm{N})$ have been obtained in the previous time steps, the superscripts $c d, c f$ and ch denote the fundamental solutions due to the continuous unit DDs, fluid source intensity and heat source intensity. In Eqs. (40) (46), we used the following definitions:

$$
\sigma_{n n(k)}^{c d}\left(\mathbf{x}-\mathbf{x}^{\prime}\right)=\left\{\begin{array}{cl}
\sigma_{n n}^{c d}\left(\mathbf{x}-\mathbf{x}^{\prime}, \Delta t\right) & \text { if } k=1 \\
\sigma_{n n}^{c d}\left(\mathbf{x}-\mathbf{x}^{\prime}, k \Delta t\right)-\sigma_{n n}^{c d}\left(\mathbf{x}-\mathbf{x}^{\prime},(k-1) \Delta t\right) & \text { if } k>1
\end{array}\right.
$$

and $\sigma_{n(k)}^{c f}\left(\mathbf{x}-\mathbf{x}^{\prime}\right), \sigma_{n(k)}^{c h}\left(\mathbf{x}-\mathbf{x}^{\prime}\right) d A\left(\mathbf{x}^{\prime}\right), p_{n(k)}^{c d}\left(\mathbf{x}-\mathbf{x}^{\prime}\right), p_{(k)}^{c f}\left(\mathbf{x}-\mathbf{x}^{\prime}\right), p_{(k)}^{c h}\left(\mathbf{x}-\mathbf{x}^{\prime}\right)$, and $T_{(k)}^{c h}\left(\mathbf{x}-\mathbf{x}^{\prime}\right)$ are defined in the similar way. 
Substituting Eqs. (55) (61) into Eqs. Eqs. (13)-(15), yields

$$
\begin{aligned}
& \sigma_{z z}(\mathbf{x}, t)=\left[\sum_{m=1}^{M} \int_{A_{e}} \sigma_{n n(1)}^{c d}\left(\mathbf{x}-\mathbf{x}^{\prime}\right) d A\left(\mathbf{x}^{\prime}\right)\right] \tilde{\mathbf{D}}_{n}(N \Delta t) \\
& +\left[\sum_{m=1}^{M} \int_{A_{e}} \mathbf{N}^{(m)} \sigma_{n(1)}^{c f}\left(\mathbf{x}-\mathbf{x}^{\prime}\right) d A\left(\mathbf{x}^{\prime}\right)\right] \tilde{\mathbf{D}}_{f}(N \Delta t)+\left[\sum_{m=1}^{M} \int_{A_{e}} \mathbf{N}^{(m)} \sigma_{n(1)}^{c h}\left(\mathbf{x}-\mathbf{x}^{\prime}\right) d A\left(\mathbf{x}^{\prime}\right)\right] \tilde{\mathbf{D}}_{h}(N \Delta t) \\
& +\sigma_{n 1}(\mathbf{x}, t)+\sigma_{n 0}(\mathbf{x}) \\
& p(\mathbf{x}, t)=\left[\sum_{m=1}^{M} \int_{A_{e}} p_{n(1)}^{c d}\left(\mathbf{x}-\mathbf{x}^{\prime}\right) d A\left(\mathbf{x}^{\prime}\right)\right] \tilde{\mathbf{D}}_{n}(N \Delta t) \\
& +\left[\sum_{m=1}^{M} \int_{A_{e}} \mathbf{N}^{(m)} p_{(1)}^{c f}\left(\mathbf{x}-\mathbf{x}^{\prime}\right) d A\left(\mathbf{x}^{\prime}\right)\right] \tilde{\mathbf{D}}_{f}(N \Delta t)+\left[\sum_{m=1}^{M} \int_{A_{e}} \mathbf{N}^{(m)} p_{(1)}^{c h}\left(\mathbf{x}-\mathbf{x}^{\prime}\right) d A\left(\mathbf{x}^{\prime}\right)\right] \tilde{\mathbf{D}}_{h}(N \Delta t) \\
& +p_{1}(\mathbf{x}, t)+p_{0}(\mathbf{x}) \\
& \quad T(\mathbf{x}, t)=\left[\sum_{m=1}^{M} \int_{A_{e}} \mathbf{N}^{(m)} T_{(1)}^{c f}\left(\mathbf{x}-\mathbf{x}^{\prime}\right) d A\left(\mathbf{x}^{\prime}\right)\right] \tilde{\mathbf{D}}_{h}(N \Delta t)+T_{1}(\mathbf{x}, t)+p_{0}(\mathbf{x})
\end{aligned}
$$

where $\sigma_{n 1}, p_{1}$ and $T_{1}$ are the sum of several terms on the right hand sides of Eq. (50) (46) and can be evaluated directly, and the other notations have been defined in the previous text. All the fundamental solutions used in these equations can be found in Cheng and Detournay [8]. Applying Eqs. (48) on all element centers, and Eqs. (49) and (50) on all element nodes at the fracture plane, we obtain

$$
\begin{gathered}
\boldsymbol{\sigma}_{n}=\mathbf{A}_{5} \tilde{\mathbf{D}}_{n}(t)+\mathbf{A}_{6} \tilde{\mathbf{D}}_{f}(t)+\mathbf{A}_{7} \tilde{\mathbf{D}}_{h}(t)+\boldsymbol{\sigma}_{n 1}+\boldsymbol{\sigma}_{n 0} \\
\tilde{\mathbf{p}}=\mathbf{A}_{8} \tilde{\mathbf{D}}_{n}(t)+\mathbf{A}_{9} \tilde{\mathbf{D}}_{f}(t)+\mathbf{A}_{10} \tilde{\mathbf{D}}_{h}(t)+\mathbf{p}_{1}+\mathbf{p}_{0} \\
\tilde{\mathbf{T}}=\mathbf{A}_{11} \tilde{\mathbf{D}}_{h}(t)+\mathbf{T}_{1}+\mathbf{T}_{0}
\end{gathered}
$$

where

$$
\begin{gathered}
\mathbf{A}_{5}=\left[\begin{array}{c}
\sum_{m=1}^{M} \int_{A_{e}} \sigma_{n n(1)}^{c d}\left(x_{1}-x^{\prime}, y_{1}-y^{\prime}, 0\right) d x^{\prime} d y^{\prime} \\
\sum_{m=1}^{M} \int_{A_{e}} \sigma_{n n(1)}^{c d}\left(x_{2}-x^{\prime}, y_{2}-y^{\prime}, 0\right) d x^{\prime} y^{\prime} \\
\vdots \\
\sum_{m=1}^{M} \int_{A_{e}} \sigma_{n n(1)}^{c d}\left(x_{M}-x^{\prime}, y_{M}-y^{\prime}, 0\right) d x^{\prime} y^{\prime}
\end{array}\right] \\
\mathbf{A}_{6}=\left[\begin{array}{c}
\sum_{m=1}^{M} \int_{A_{e}} \mathbf{N}^{(m)} \sigma_{n(1)}^{c f}\left(x_{1}-x^{\prime}, y_{1}-y^{\prime}, 0\right) d x^{\prime} d y^{\prime} \\
\sum_{m=1}^{M} \int_{A_{e}} \mathbf{N}^{(m)} \sigma_{n(1)}^{c f}\left(x_{2}-x^{\prime}, y_{2}-y^{\prime}, 0\right) d x^{\prime} d y^{\prime} \\
\vdots \\
\sum_{m=1}^{M} \int_{A_{e}} \mathbf{N}^{(m)} \sigma_{n(1)}^{c f}\left(x_{M}-x^{\prime}, y_{M}-y^{\prime}, 0\right) d x^{\prime} d y^{\prime}
\end{array}\right]
\end{gathered}
$$




$$
\begin{aligned}
& \mathbf{A}_{7}=\left[\begin{array}{c}
\sum_{m=1}^{M} \int_{A_{e}} \mathbf{N}^{(m)} \sigma_{n(1)}^{c h}\left(x_{1}-x^{\prime}, y_{1}-y^{\prime}, 0\right) d x^{\prime} d y^{\prime} \\
\sum_{m=1}^{M} \int_{A_{e}} \mathbf{N}^{(m)} \sigma_{n(1)}^{c h}\left(x_{2}-x^{\prime}, y_{2}-y^{\prime}, 0\right) d x^{\prime} d y^{\prime} \\
\vdots \\
\sum_{m=1}^{M} \int_{A_{e}} \mathbf{N}^{(m)} \sigma_{n(1)}^{c h}\left(x_{M}-x^{\prime}, y_{M}-y^{\prime}, 0\right) d x^{\prime} d y^{\prime}
\end{array}\right] \\
& \mathbf{A}_{8}=\left[\begin{array}{c}
\sum_{m=1}^{M} \int_{A_{e}} p_{n(1)}^{c d}\left(x_{1}-x^{\prime}, y_{1}-y^{\prime}, 0\right) d x^{\prime} d y^{\prime} \\
\sum_{m=1}^{M} \int_{A_{e}} p_{n(1)}^{c d}\left(x_{2}-x^{\prime}, y_{2}-y^{\prime}, 0\right) d x^{\prime} y^{\prime} \\
\vdots \\
\sum_{m=1}^{M} \int_{A_{e}} p_{n(1)}^{c d}\left(x_{l}-x^{\prime}, y_{l}-y^{\prime}, 0\right) d x^{\prime} y^{\prime}
\end{array}\right] \\
& \mathbf{A}_{9}=\left[\begin{array}{c}
\sum_{m=1}^{M} \int_{A_{e}} p_{(1)}^{c f}\left(x_{1}-x^{\prime}, y_{1}-y^{\prime}, 0\right) d x^{\prime} d y^{\prime} \\
\sum_{m=1}^{M} \int_{A_{e}} p_{(1)}^{c f}\left(x_{2}-x^{\prime}, y_{2}-y^{\prime}, 0\right) d x^{\prime} y^{\prime} \\
\vdots \\
\sum_{m=1}^{M} \int_{A_{e}} p_{(1)}^{c f}\left(x_{l}-x^{\prime}, y_{l}-y^{\prime}, 0\right) d x^{\prime} y^{\prime}
\end{array}\right] \\
& \mathbf{A}_{10}=\left[\begin{array}{c}
\sum_{m=1}^{M} \int_{A_{e}} p_{(1)}^{c h}\left(x_{1}-x^{\prime}, y_{1}-y^{\prime}, 0\right) d x^{\prime} d y^{\prime} \\
\sum_{m=1}^{M} \int_{A_{e}} p_{(1)}^{c h}\left(x_{2}-x^{\prime}, y_{2}-y^{\prime}, 0\right) d x^{\prime} y^{\prime} \\
\vdots \\
\sum_{m=1}^{M} \int_{A_{e}} p_{(1)}^{c h}\left(x_{l}-x^{\prime}, y_{l}-y^{\prime}, 0\right) d x^{\prime} y^{\prime}
\end{array}\right] \\
& \mathbf{A}_{11}=\left[\begin{array}{c}
\sum_{m=1}^{M} \int_{A_{e}} T_{(1)}^{c h}\left(x_{1}-x^{\prime}, y_{1}-y^{\prime}, 0\right) d x^{\prime} d y^{\prime} \\
\sum_{m=1}^{M} \int_{A_{e}} T_{(1)}^{c h}\left(x_{2}-x^{\prime}, y_{2}-y^{\prime}, 0\right) d x^{\prime} y^{\prime} \\
\vdots \\
\sum_{m=1}^{M} \int_{A_{e}} T_{(1)}^{c h}\left(x_{l}-x^{\prime}, y_{l}-y^{\prime}, 0\right) d x^{\prime} y^{\prime}
\end{array}\right]
\end{aligned}
$$




$$
\begin{aligned}
& \boldsymbol{\sigma}_{n 1}=\left\{\begin{array}{c}
\sigma_{n 1}\left(x_{1}, y_{1}, 0\right) \\
\sigma_{n 1}\left(x_{2}, y_{2}, 0\right) \\
\vdots \\
\sigma_{n 1}\left(x_{M}, y_{M}, 0\right)
\end{array}\right\} \\
& \boldsymbol{\sigma}_{n 0}=\left\{\begin{array}{c}
\sigma_{n 0}\left(x_{1}, y_{1}, 0\right) \\
\sigma_{n 0}\left(x_{2}, y_{2}, 0\right) \\
\vdots \\
\sigma_{n 0}\left(x_{M}, y_{M}, 0\right)
\end{array}\right\} \\
& \mathbf{p}_{1}=\left\{\begin{array}{c}
p_{1}\left(x_{1}, y_{1}, 0\right) \\
p_{1}\left(x_{2}, y_{2}, 0\right) \\
\vdots \\
p_{1}\left(x_{l}, y_{l}, 0\right)
\end{array}\right\} \\
& \mathbf{p}_{0}=\left\{\begin{array}{c}
p_{0}\left(x_{1}, y_{1}, 0\right) \\
p_{0}\left(x_{2}, y_{2}, 0\right) \\
\vdots \\
p_{0}\left(x_{l}, y_{l}, 0\right)
\end{array}\right\} \\
& \mathbf{T}_{1}=\left\{\begin{array}{c}
T_{1}\left(x_{1}, y_{1}, 0\right) \\
T_{1}\left(x_{2}, y_{2}, 0\right) \\
\vdots \\
T_{1}\left(x_{l}, y_{l}, 0\right)
\end{array}\right\} \\
& \mathbf{T}_{0}=\left\{\begin{array}{c}
T_{0}\left(x_{1}, y_{1}, 0\right) \\
T_{0}\left(x_{2}, y_{2}, 0\right) \\
\vdots \\
T_{0}\left(x_{l}, y_{l}, 0\right)
\end{array}\right\}
\end{aligned}
$$

in which $l$ is the total number of element nodes and $M$ is the total number of elements in the mesh for the fracture surface. All these matrices and vectors can be evaluated directly. It is observed that we have $4 l+M$ unknowns $\left(\tilde{\mathbf{p}}, \tilde{\mathbf{T}}, \tilde{\mathbf{D}}_{f}, \tilde{\mathbf{D}}_{h}\right.$ and $\left.\tilde{\mathbf{D}}_{n}\right)$ in the present problems. Eqs. (32) and (33) contains $2 l$ linear equations and Eqs. (51)-(53) contains $2 l+M$ linear equations. The present problem is fully described by these equations.

In this work, the space integrals over the elements are performed numerically. We adopt the numerical algorithm proposed by Guiggiani et al. [12] to evaluate the integrals that become hypersingular at the fracture surface. Apart from the treatment of the hypersingular integrals, the strongly singular kernel function can also be integrated with the same numerical algorithm [13]. The weakly integral singularity is treated by using the polar coordinate transformation method [14]. 


\subsection{Numerical Procedures for Flow \& Deformation in Natural Fracture}

When flow is coupled to fracture deformation and heat transport, the fracture is discretized into a number of four-noded quadrilateral elements, and the equations (32), (33), and (51) (53) are used to simulate the fully coupled fluid injection/extraction phenomena through a natural fracture. It is assumed that the fracture is a joint and its deformation is linear elastic. The normal fracture stiffness for any element $i$ is denoted as

$\stackrel{i}{K}_{n}$. In the simulation process, elements can be either open or closed. The following separation criterion is adopted to judge whether the element $i$ is closed or open:

$$
\tilde{\sigma}_{n}^{\prime}=-\stackrel{i}{c} \operatorname{cotan} \dot{i}_{\text {eff }}
$$

When this condition is satisfied the joint element is cracked (separated); and if element $i$

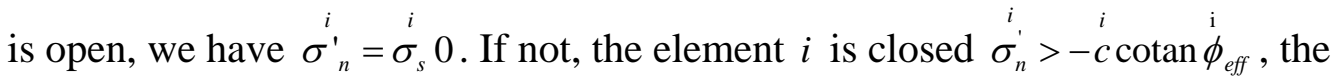
incremental increase or decrease of the normal effective stress for element $i$ can be calculated by

$$
\Delta \sigma_{n}^{i}=-\stackrel{i}{K_{n}} \stackrel{i}{D}_{n}
$$

The current transient problem is solved by marching in the time domain. Within any time step $K$, iterations areused to take into account the coupling between the fluid flow and heat transport in the fracture, and the reaction of the reservoir matrix. We begin with $j=1$ iteration. We assume the fracture aperture vector $\mathbf{w}_{K}^{1}$ in $j=1$ iteration to be equal to the calculated fracture apertures in the last time step if $K>1$ or the initial fracture aperture $\mathbf{w}_{0}$ if $K=1$, where the subscript and superscript denote the time step and and the iteration in the current time step, respectively. Solving Eqs. (32), (33), and (51) (53), we can obtain $\tilde{\mathbf{D}}_{n}(t), \tilde{\mathbf{D}}_{f}(t), \tilde{\mathbf{D}}_{h}(t), \tilde{\mathbf{p}}(t)$ and $\tilde{\mathbf{T}}(t)$ for the current iteration $j$ within time step $K$. Then a new fracture aperture vector which is equal to $\mathbf{w}_{0}+\tilde{\mathbf{D}}_{n}$ and denoted as $\mathbf{w}_{K}^{j+1} \cdot \mathbf{w}_{K}^{j+1}$ will be used in the next $j+1$ iteration. The iterative process continues until the following convergent condition

$$
\left\|\mathbf{w}_{K}^{k}-\mathbf{w}_{K}^{k-1}\right\| /\left\|\mathbf{w}_{K}^{k-1}\right\|<\mathrm{TOL}
$$

is satisfied, where TOL is a prescribed tolerance. We use TOL $=1 \%$ for the numerical examples presented in this paper. Thereafter, the simulation marches into the next time step $K+1$.

Given fracture apertures, Eqs. (32), (33), and (51) (57) need to be solved in every iteration. As the rock temperature is independent on the stress state, we substitute Eq. (53) into Eq. (38) and then obtain

$$
\left(\mathbf{A}_{3} \mathbf{A}_{11}+\mathbf{A}_{4}\right) \tilde{\mathbf{D}}_{h}=-\mathbf{A}_{3}\left(\mathbf{T}_{1}+\mathbf{T}_{0}\right)
$$

in which $\tilde{\mathbf{D}}_{h}$ could be solved directly. Thereafter, $\tilde{\mathbf{T}}$ could be obtained by substituting $\tilde{\mathbf{D}}_{h}$ into Eq. (53). 
The procedure for solving $\tilde{\mathbf{p}}, \tilde{\mathbf{D}}_{f}$ and $\tilde{\mathbf{D}}_{n}$ are as follows. Substituting Eq. (52) into Eq. (32), we have

$$
\mathbf{A}_{1} \mathbf{A}_{8} \tilde{\mathbf{D}}_{n}+\left(\mathbf{A}_{1} \mathbf{A}_{9}+\mathbf{A}_{2}\right) \tilde{\mathbf{D}}_{f}=\mathbf{B}_{1}-\mathbf{A}_{1} \mathbf{A}_{10} \tilde{\mathbf{D}}_{h}-\mathbf{A}_{1}\left(\mathbf{p}_{1}+\mathbf{p}_{0}\right)
$$

where all the terms on the right hand side have been evaluated or solved.

Eq. (51) can be rewritten as

$$
\mathbf{A}_{5} \tilde{\mathbf{D}}_{n}(t)+\mathbf{A}_{6} \tilde{\mathbf{D}}_{f}(t)=\boldsymbol{\sigma}_{n}-\boldsymbol{\sigma}_{n 0}-\boldsymbol{\sigma}_{n 1}-\mathbf{A}_{7} \tilde{\mathbf{D}}_{h}(t)
$$

where $\boldsymbol{\sigma}_{n}$ should be evaluated differently for closed or opened elements. For convenience of description, we use $\dot{\sigma}_{n}$ to denote the $i^{\text {th }}$ component of $\boldsymbol{\sigma}_{n}$ and $\dot{\sigma}_{n 0}$ to denote the $i^{\text {th }}$ component of $\boldsymbol{\sigma}_{n 0}$. Then, if element $i$ is closed, we have

$$
\begin{aligned}
& i \\
& \sigma_{n}-\sigma_{n 0}
\end{aligned}=\left(\begin{array}{cc}
i & i \\
\sigma_{n}^{\prime} & p
\end{array}\right)-\left(\begin{array}{cc}
i & i \\
\sigma_{n 0}^{\prime} & +p_{0}
\end{array}\right)=\left(\begin{array}{cc}
i & i \\
p-p_{0}
\end{array}\right)+\left(\begin{array}{cc}
i & i \\
\sigma_{n}^{\prime} & -\sigma_{n 0}^{\prime}
\end{array}\right)=\left(\begin{array}{cc}
i & i \\
p-p_{0}
\end{array}\right)-i_{n} \quad \stackrel{i}{D}_{n}
$$

while if element $i$ is open, we have

$$
i_{n}-\sigma_{n 0}=\stackrel{i}{i}-\sigma_{n 0}
$$

Eqs. (88) or (89) can be used to evaluate $\boldsymbol{\sigma}_{n}-\boldsymbol{\sigma}_{n 0}$, causing the existence of the unknowns $\tilde{\mathbf{p}}$ on the right hand side of Eq. (87). However, $\tilde{\mathbf{p}}$ can be removed by using Eq. (52). Now only $\tilde{\mathbf{D}}_{n}$ and $\tilde{\mathbf{D}}_{f}$ are involved in Eqs. (71) and (72) and they can be determined by solving a linear equation system. Thereafter, $\tilde{\mathbf{p}}$ can be obtained by substituting $\tilde{\mathbf{D}}_{n}, \tilde{\mathbf{D}}_{f}$ and $\tilde{\mathbf{D}}_{h}$ into Eq. (52). In the current version of the fluid flow and rock deformation coupling, fracture slip is not considered but this phenomenon is included when flow is not coupled to fracture deformation. 


\section{References:}

1. Sherburn, S. 1984. Seismic monitoring during a cold water injection experiment, Wairakei Geothermal Field: preliminary results, In: Proc. 6th New Zealand Geothermal Workshop, 6, 129-133.

2. Stark, M.A. 1990. Imaging injected water in the Geysers reservoir using micro-earthquakes data. In: Geothermal Res. Council Trans, 14(II): 1697-1704.

3. Mossop, A. 2001. Seismicity, Subsidence and strain at the Geysers geothermal field. Ph.D. Dissertation, Stanford University.

4. Ghassemi, A., S. Tarasovs, and Cheng. A.D.-H. 2003. An Integral equation method for modeling three-dimensional heat extraction from a fracture in hot dry rock. Int. J. Num. \& Anal. Methods in Geomech. 27: 12, 989-1004.

5. Cheng, A.-D., A. Ghassemi, and Detournay, E. 2001. A two-dimensional solution for heat extraction from a fracture in hot dry rock. Int. J. Numer. Analy. Meth. Geomech. 25: 13271338.

6. Ghassemi A. and Zhang Q. 2006. Poro-thermoelastic response of a stationary crack using the displacement discontinuity method. ASCE J. Eng. Mech., 132(1), 26-33.

7. Lowell R.P. 1976. Comments on 'Theory of heat extraction from fracture hot dry rock' by AC Gringaten, PA Witherspoon, Y Ohnishi. J. Geoph. Res. 81:359-360.

8. Cheng A.H.-D. and Detournay E. 1998. On singular integral equations and fundamental solutions of poroelasticity. Int. J. Solids Struc. 35(35):4521-4555.

9. Ghassemi A., Tarasovs S., and Cheng A.H.-D. 2007. A three-dimensional study of the effects of thermo-mechanical loads on fracture slip in enhanced geothermal reservoir. Int. J. Rock Mechanics \& Min Sci., 44 , pp. 1132-1148.

10. Brooks A.N. and Hughes T.J.R. 1982. Streamline upwind/Petrov-Galerkin formulations for convection dominated flows with particular emphasis on the incompressible Navier-Stokes equations. Computer Methods in Applied Mechanics and Engineering. 32:199-259.

11. Dargush G..F. and Banerjee P.K. 1989. A time domain boundary element method for poroelasticity. Int. J. Numer. Methods Eng., 28, 2423-2449.

12. Guiggiani M. and Gigante A. 1990. A general algorithm for multidimensional Cauchy principal value integrals in the boundary element method. J. Appl. Mech., 57, pp. 906-915.

13. Guiggiani M., Krishnasamy, G., Rudolphi, T.J., and Rizzo, F.J. 1992. A general algorithm for the numerical solution of hypersingular boundary integral equations. J. Appl. Mech., 59, pp. 604-614.

14. Beer G. 2001. Programming the boundary element method: an introduction for engineers. Wiley. 


\section{Applications \& Analysis: Poro-thermoelasticity}

The theoretical developments of Chapters 2-4 will now be used to study a number of problems pertaining to EGS development. These include 2D poro-thermoelatic analysis of a fracture and fracture propagation, simulation of injection/extraction in fractured reservoir, 3D poroelastic analysis of natural fractures, and combined poro- and thermoelastic study of injection/extraction into planar fractures. To the extent possible, the input parameters used in the analyses correspond to the Coso geothermal field.

\subsection{Poro-thermoelastic Analysis of Fracture Opening}

In this section, we apply the poro-thermoelastic DD method to the problem of a stationary crack in a geothermal reservoir to examine the relative importance of various mechanisms in fracture propagation and to highlight the coupled effects in geothermal reservoirs. The validity of the DD approach for poro-thermoelastic problems has been demonstrated in Ghassemi and Zhang [1] where the numerical model has been compared to analytical results indicating a very good agreement. The problem under consideration is the opening of a suddenly pressurized and cooled crack of length $2 \mathrm{~L}$ (shown in Figure 5.1). This problem of fracture pressurization by a cooler fluid under pressure, $P$, may be decomposed into three sub-problems corresponding to three modes of loading: a normal stress loading, a pore pressure loading, and a temperature loading:

Mode 1: $\sigma_{n}(x, t)=-P H(t) ; \quad p(x, t)=0 ; \quad T(x, 0)=0$

Mode $2: \sigma_{n}(x, t)=0 ; \quad p(x, t)=P H(t) ; \quad T(x, 0)=0$

Mode $3: \sigma_{n}(x, t)=0 ; \quad p(x, t)=0 ; \quad T(x, 0)=T_{c} H(t)$

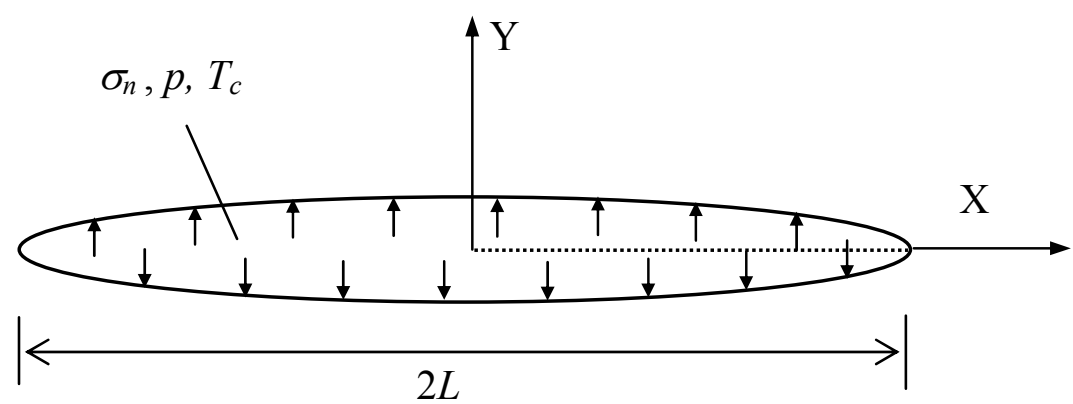

Figure 5.1. A uniformly pressurized, cooled crack.

where $\mathrm{H}(\mathrm{t})$ denotes the Heaviside step function. The initial conditions for both problems are zero stress and pore pressure everywhere. 
Table 1. Input parameters

\begin{tabular}{|l|l|}
\hline Shear modulus $G$ & $16 \mathrm{GPa}$ \\
\hline Biot's coefficient $\alpha$ & 0.443 \\
\hline Drained Poisson's ratio $v$ & 0.25 \\
\hline Undrained Poisson's Ratio $v_{u}$ & 0.34 \\
\hline Permeability k, & $4.053 \times 10^{-08} \mathrm{~m} / \mathrm{sec}$ \\
\hline Porosity $\phi$ & 0.01 \\
\hline Thermal exp. coef. solid $\alpha_{m}$ & $2.4 \times 10^{-5} \mathrm{~K}^{-1}$ \\
\hline Thermal exp. coef. fluid $\alpha_{f}$ & $3 \times 10^{-4} \mathrm{~K}^{-1}$ \\
\hline Thermal diffusivity c ${ }^{T}$ of intact rock & $5 \times 10^{-6} \mathrm{~m}^{2} / \mathrm{s}$ \\
\hline Thermal diffusivity c ${ }^{f}$ of intact rock & $6.48 \times 10^{-5} \mathrm{~m}^{2} / \mathrm{s}$ \\
\hline Fluid density & $1 \times 10^{3} \mathrm{Kg} / \mathrm{m}^{3}$ \\
\hline Heat capacity of fluid & $4200 \mathrm{~J} /(\mathrm{kg} . \mathrm{K})$ \\
\hline heat capacity of rock & $790 \mathrm{~J} /(\mathrm{kg} . \mathrm{K})$ \\
\hline Thermal conductivity of fluid & $0.6 \mathrm{~J} /(\mathrm{s} . \mathrm{m} . \mathrm{K})$ \\
\hline
\end{tabular}

where $\mathrm{H}(\mathrm{t})$ denotes the Heaviside step function. The initial conditions for each subproblem are zero stress, pore pressure, and a temperature of To. We will focus on the induced quantities with the aim of investigating various mechanisms. The result of Mode 1 loading is shown in Figure 5.2. This mode of loading is responsible for the opening of the fracture. At time $\mathrm{t}=0^{+}$, the fracture opens according to the solution of Sneddon [2] with undrained material properties:

$$
W(x)=-\frac{2 P L\left(1-v_{u}\right)}{G}\left(1-\frac{x^{2}}{L^{2}}\right)
$$

As time increases the crack opening also increases, approaching the steady-state solution given by the previous equation with drained material properties. This stage of rock deformation is referred to as the drained stage. The crack opening is a maximum at this stage reflecting the softer material behavior. Figure 5.2 shows the normalized width evolution (with respect to Eq. 2 as a function of normalized time as defined by $\tau=\frac{t c_{f}}{L^{2}}$.

The fracture response in Mode 2 is illustrated in Figure 5.3. The crack progressively closes, starting from a zero value and reaching a final closure value of 0.22 given by: $\left(D_{n}\right)_{\max }=2 \eta\left(1-v_{u}\right)$; where $\eta$ is the poroelastic stress parameter defined as [e.g., 3]:

$$
\eta=\frac{3\left(v_{u}-v\right)}{2 B\left(1+v_{u}\right)(1-v)}
$$

Because the two crack surfaces cannot overlap, this closure is physically possible only if the crack remains open under combinations of loading modes 1,2 , and 3 . The numerical model overestimates the fracture opening by about 5 per cent. This may be attributed to 
the used of constant elements and also the fact that the very small time fracture response could not be captured. This deficiency has been removed as will shown later.

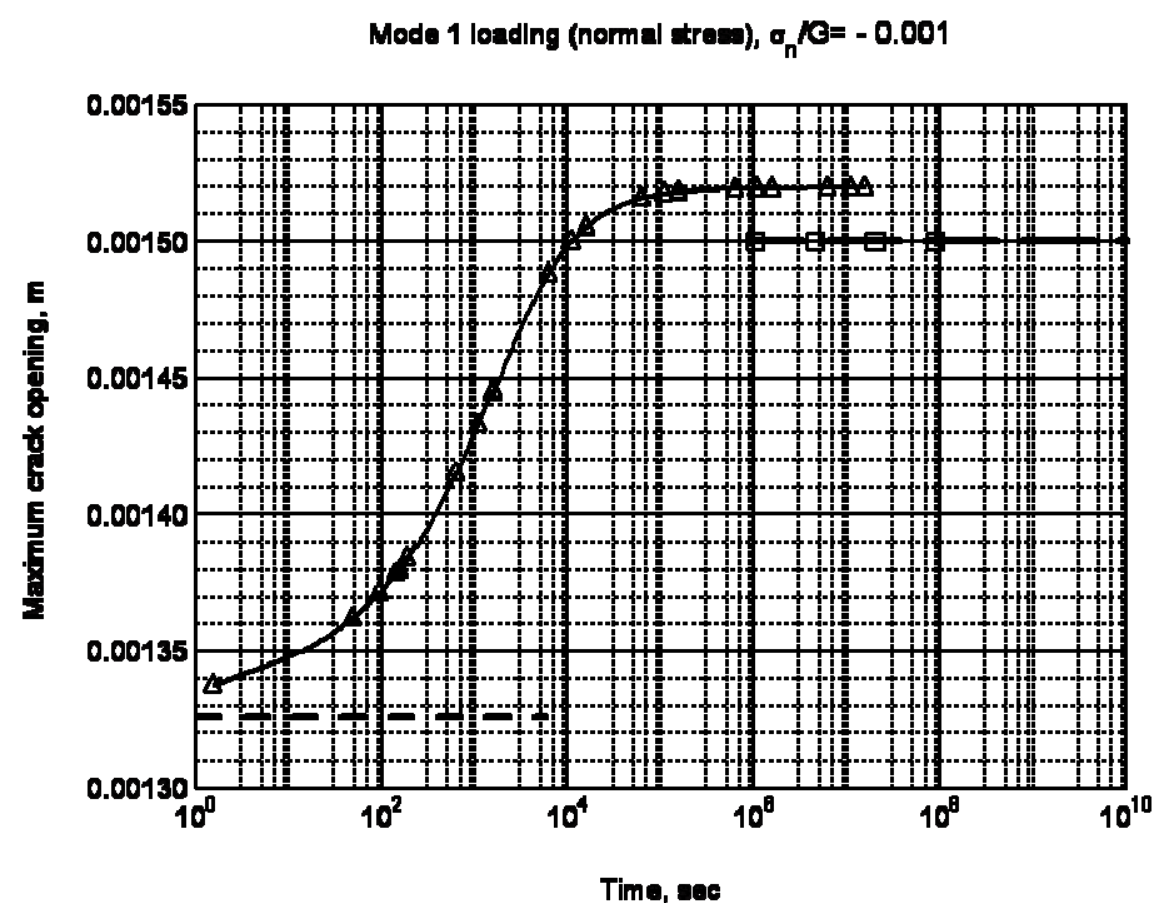

Figure 5.2. Magnitude of crack opening due to an applied stress.

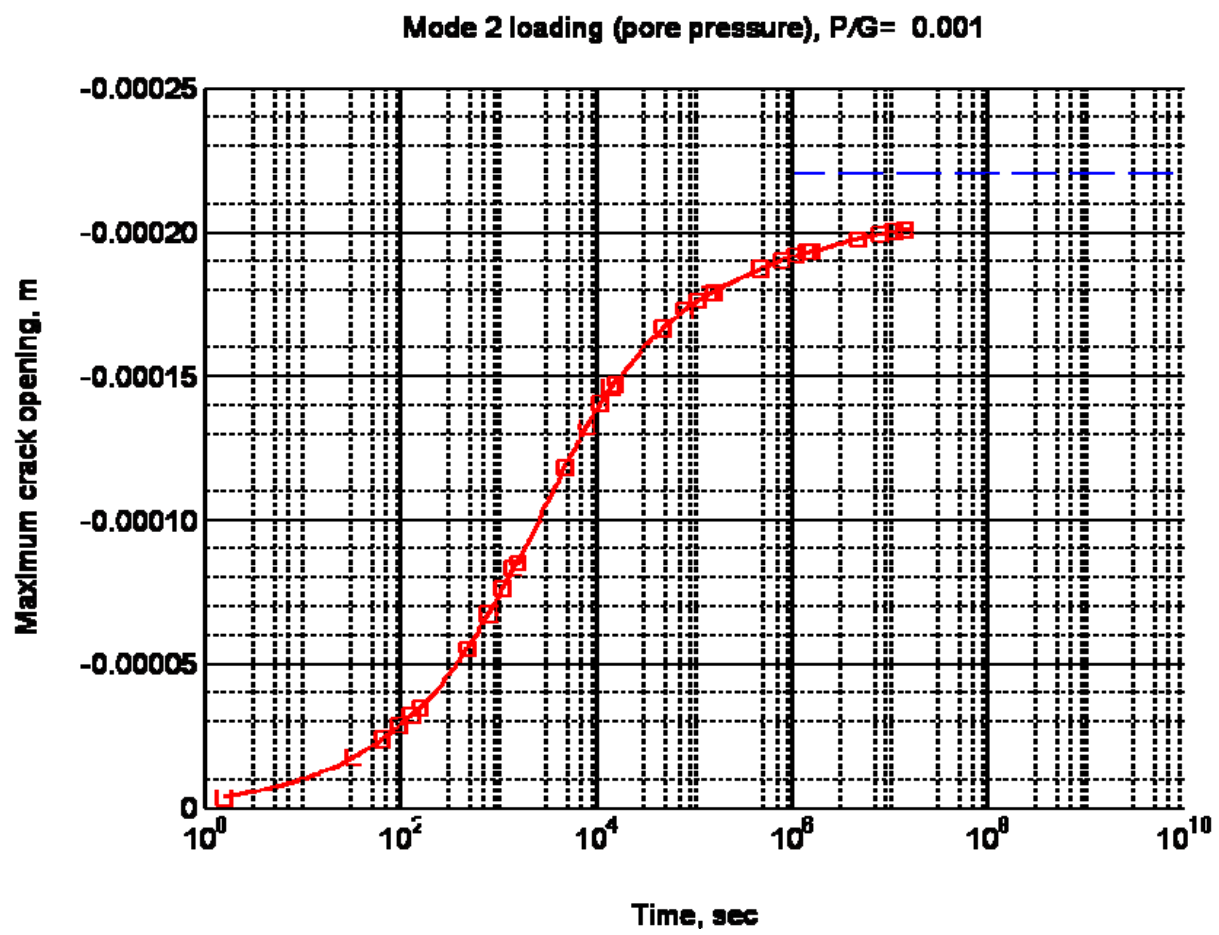

Figure 5.3. Normalized crack opening due to pore pressure loading. 
The Mode 3 fracture response as a function of time is illustrated in Figure 4. It can be seen that the cooling the crack surfaces and the surrounding rock results in opening of the fracture. This is the opposite of the effect of fluid invasion into the rock mass that tends to close the crack. The fracture opening approaches the asymptotic value shown in red which is obtained by applying the thermal stresses that are generated by cooling, to the surface of a Griffith crack. For a two dimensional geometry (plane strain) it can be shown analytically that the stresses induced on the fracture faces (at steady-state) are given by: $\sigma_{x x}=\sigma_{x x}=-\frac{\beta_{s} E \Delta T}{6(1-v)}$, where $\beta s$ is the volumetric thermal expansion coefficient of the bulk solid under constant pore pressure.

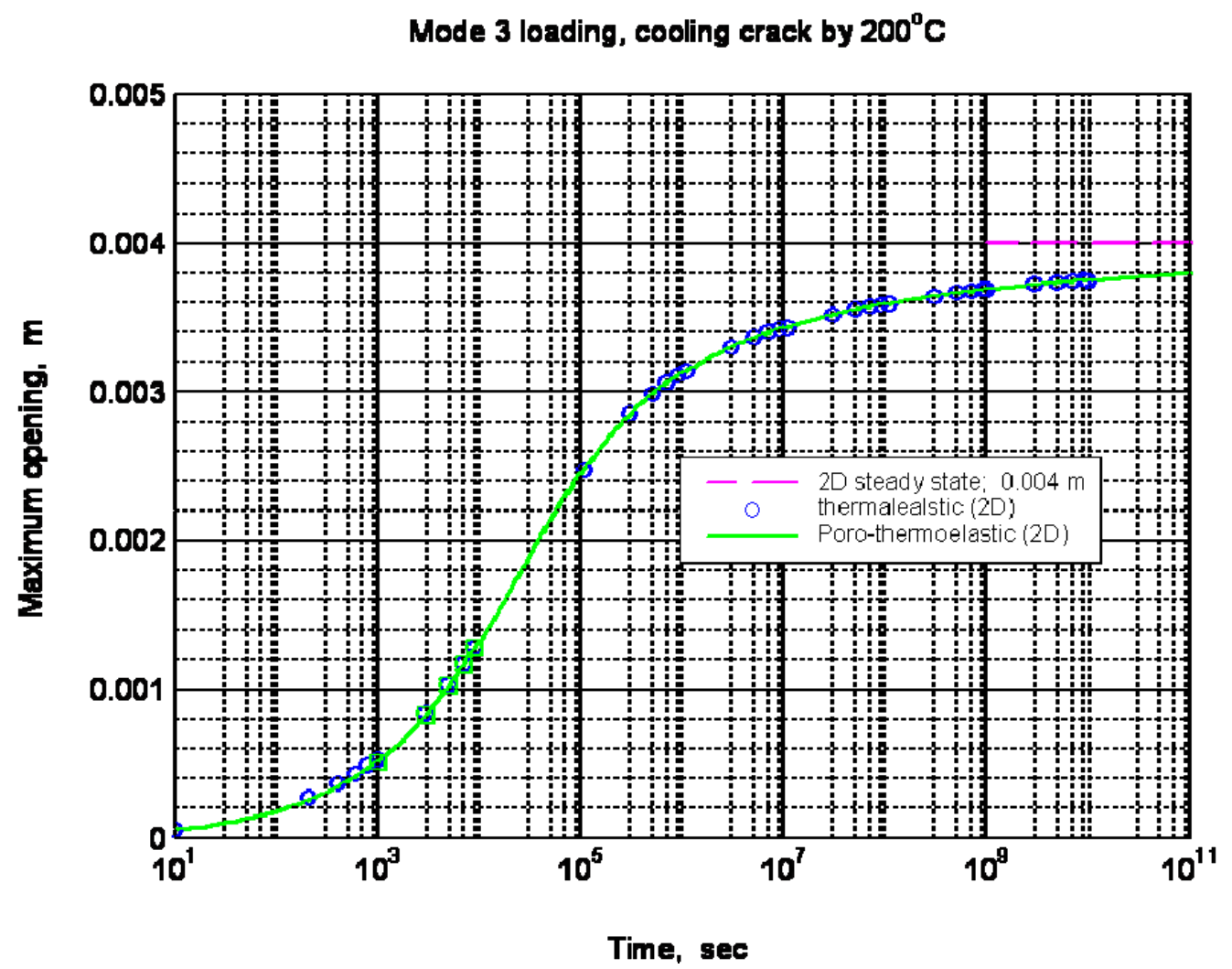

Figure 5.4. Maximum crack opening due to cooling, also shown is the steady-state analytical value.

By comparing the fracture opening profiles as a function of time for various modes, it can be observed that the contributions of Modes 1 and 2 to fracture propagation are dominant for conventional hydraulic fracturing, but they become negligible in comparison to cooling during long term stimulation. The contribution of thermal stresses is significant in fracture initiation, and in circulation operations that are carried out over a long period of time such that the thermal front has had sufficient time to develop. It should be noted that the impact of Mode 2 can significantly increase if one takes into consideration the increase in rock permeability due to thermal cracking. 


\subsubsection{Improved solution for short time and long time crack response}

The previous simulation were carried out at small time steps, however, it was not possible to capture the very early time response of the crack. To do so, a number of improvements were implemented in the code including the possibility of using multiple time increments during the solution and also deriving the short tome solution of the time-dependent part of the solution.

Using the improved code, we simulated the pressurized, cooled crack problem again. For this problem we use a crack length of $1 \mathrm{~m}$ with all material properties are the same as in Table 2. However, it is assumed that the in-situ stresses are $S_{\mathrm{H}}=30 \mathrm{MPa} ; S_{\mathrm{h}}=10 \mathrm{MPa}$, and the crack is cooled from $200^{\circ}$ to 0 while pressurized with at a level of $30 \mathrm{MPa}$.

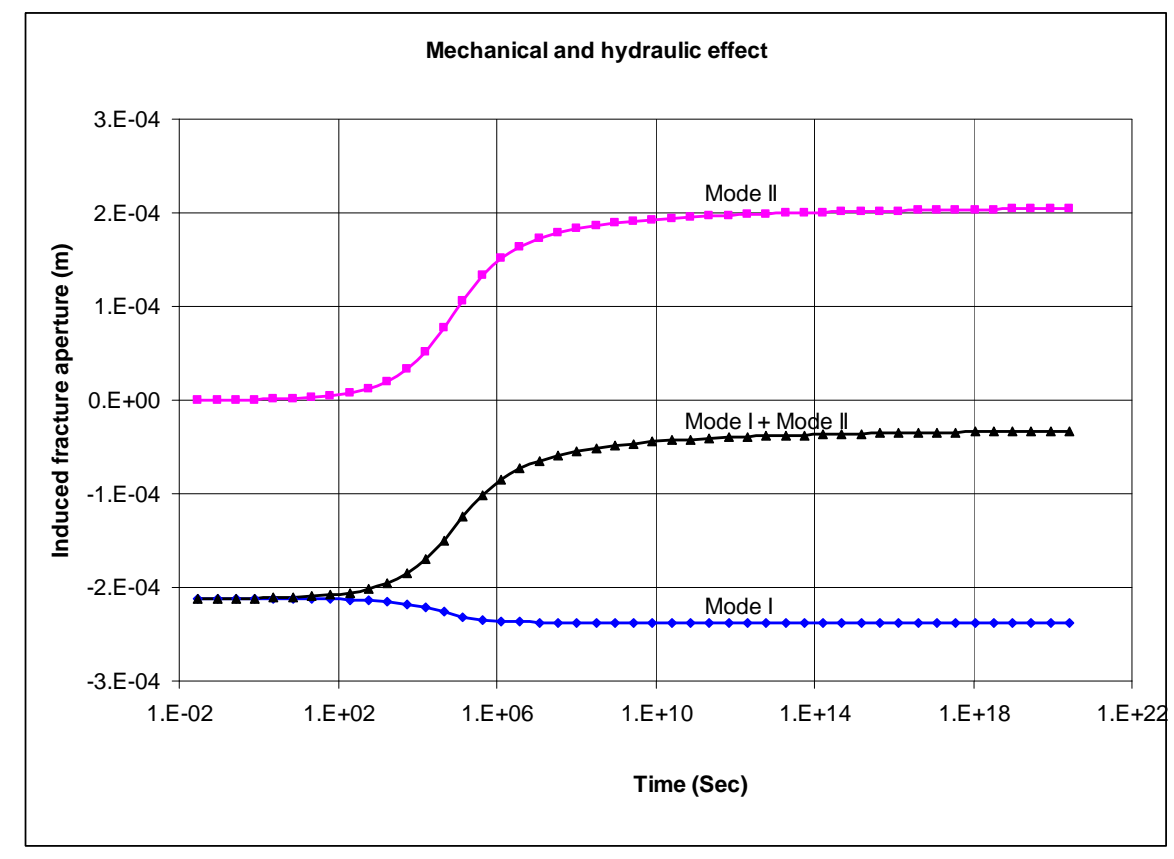

Figure 5.5. Crack opening for 3 loading modes using the improved DD method. Note the smooth transition of DD to a small time of $\mathrm{t}=0.01 \mathrm{sec}$. Crack opening is shown as negative. 


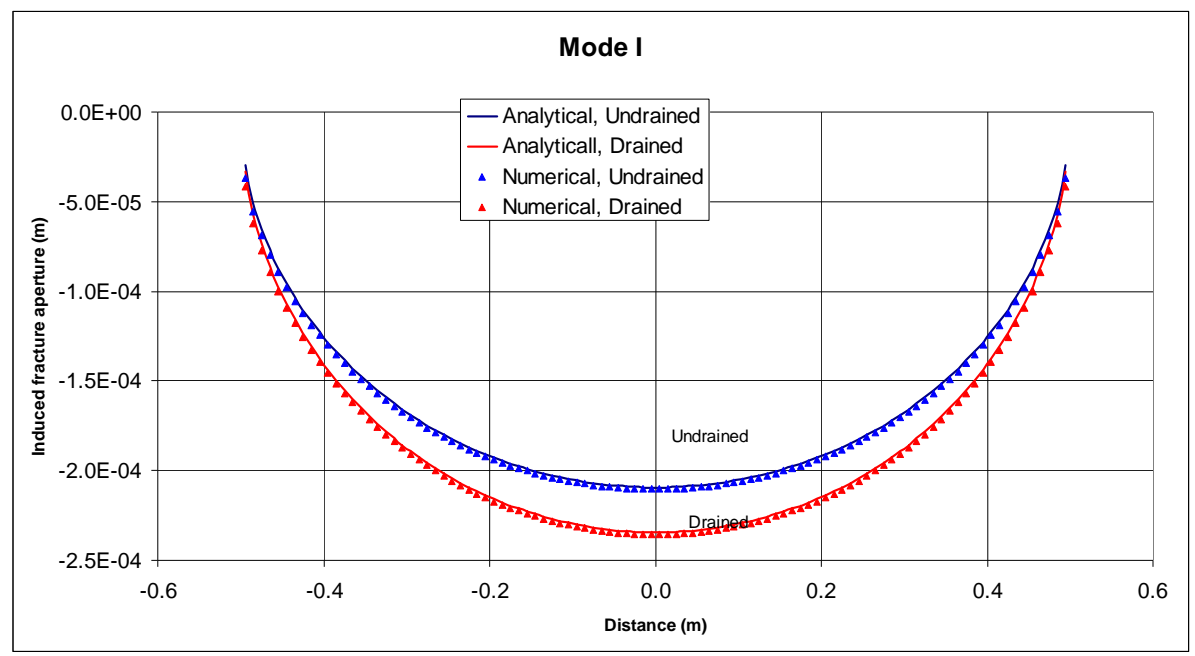

Figure 5.6. Comparison of drained and un-drained solutions with analytical results.

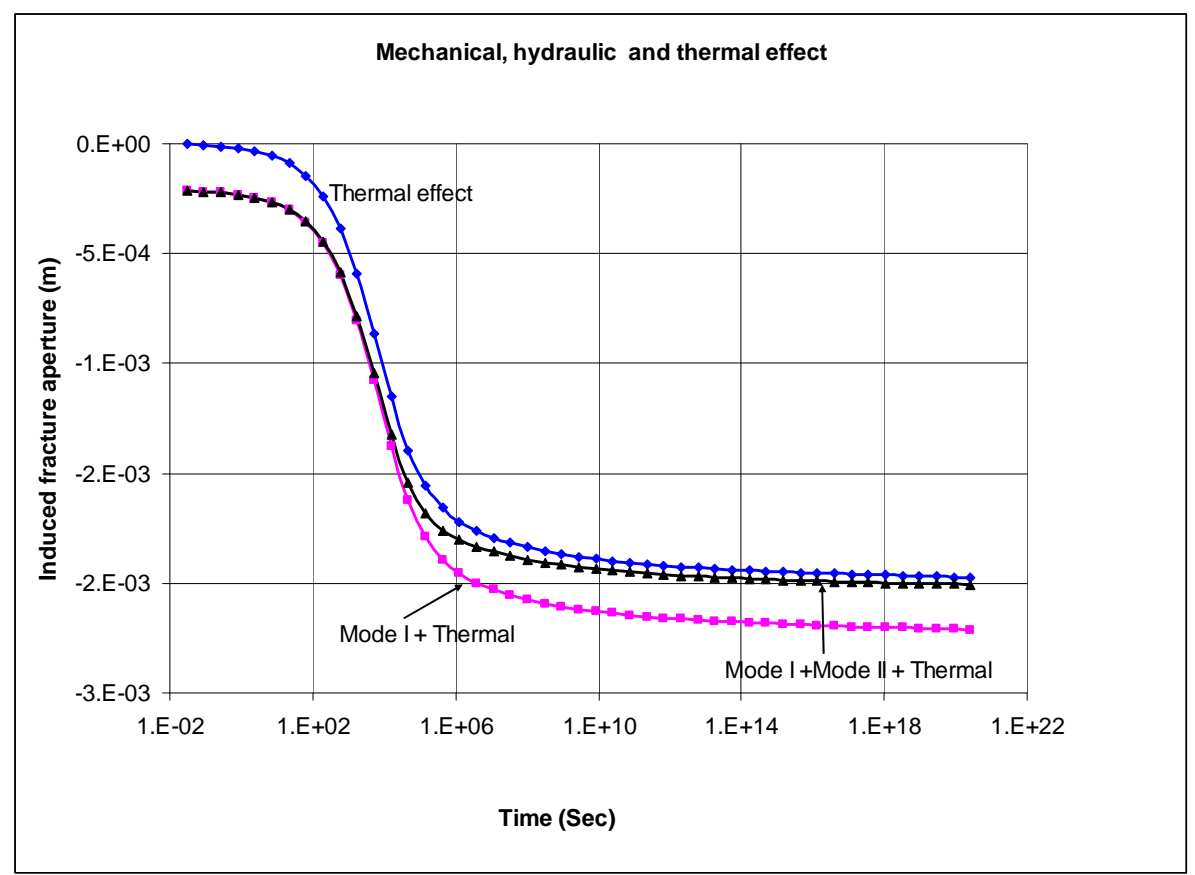

Figure 5.7. Fracture opening caused by combined loading modes. Note the dominance of mode 1 during short times.

It can bee seen that the model captures the drained and undrained responses rather well when using 100 element; using 10 elements results in approximately 4-5\% of error. Figure 5.7 illustrates the fracture opening caused by combined loading modes. It can be seen that mode 1 loading is dominant during short times while thermal stresses dominate at long times. This is to be expected as in this case the thermal diffusivity of the rock is much smaller than rock's fluid diffusivity so that the cooling response takes a much larger time to fully develop. 


\subsection{Modeling Fracture Propagation}

In this section, we consider the problem of fracture propagation while considering the impact of fluid diffusion and poroelsatic stresses. In this work, we do not consider the impact of thermal stresses as they develop very gradually not affecting the propagation. Also, the coupling between temperature and pore pressure is not considered at this time. The model uses a partially-coupled poroelastic formulation to calculate the fracture opening. The structural criterion described in the previous chapter is used to model crack propagation.

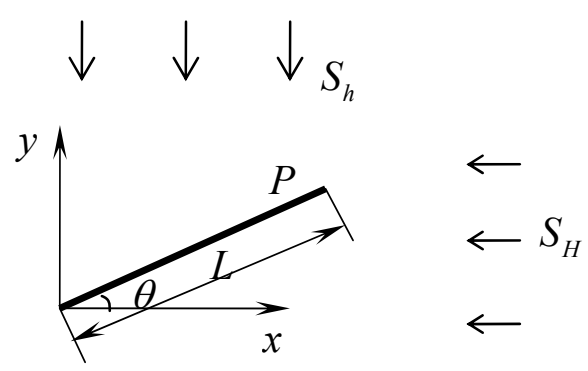

Figure 5.8. A pressurized crack under biaxial stresses in an infinite poroelastic medium.

As an example consider a line crack in an infinite poroelastic medium under the planestrain condition. The crack length is $L=0.2 \mathrm{~m}$ and is inclined $\theta$ degree with respect to $x$ axis. The far field minimum and maximum principal stresses are $\mathrm{S}_{\mathrm{h}}$ and $\mathrm{S}_{\mathrm{H}}$, respectively. The crack surfaces are suddenly pressurized from $\mathrm{t}=0^{+}$by an internal fluid with a constant pressure $P$. The crack is allowed to propagate from one crack tip. The input parameters are for Westerly granite, shown in Table 2 . The propagation trajectories are simulated for different crack propagation speeds, far field stresses, rock cohesion and internal fluid pressures in order to investigate the poroelastic effects on the fracture propagation behaviors. The results are plotted from Figures 5.9-5.11 and are discussed below.

There are three characteristic lengths for the crack propagation problem in a poroelastic medium [4], these are $c^{f} / v$ - the ratio of the diffusivity to the crack propagation velocity, $L$ - the length over which the crack face load is applied and $\omega$ - the size of the breakdown zone. Accordingly, three different propagation regimes can be identified which are categorized by different values of $c^{f} / v, \mathrm{~L}$ and $\omega$ corresponding to different crack propagation speed. In the fast regime, $L>>\omega>>c^{f} / v$, the crack propagates at a very fast speed, so the pore pressure at the crack tip does not have time to diffuse. In the intermediate regime, $L>>c^{f} / v>>\omega$, the crack propagates at a considerable speed relative to the physical length of the crack. And in the slow regime, $c^{f} / v>>L>>\omega$, the crack propagates at such a low speed that the pore pressure is drained in the tip region. 
Table 2. Input Parameters

\begin{tabular}{|c|l|l|l|}
\hline$G$ & Shear modulus & $1.5 \times 10^{04}$ & $\mathrm{MPa}$ \\
\hline$\phi$ & Internal fiction angle & 30 & $\circ$ \\
\hline$c$ & Cohesion & & $\mathrm{MPa}$ \\
\hline$\sigma_{t}$ & Tensile strength & 2.0 & $\mathrm{MPa}$ \\
\hline$P$ & Pressure & $1.5 \times 10^{01}$ & $\mathrm{MPa}$ \\
\hline$v$ & Poisson's ratio & 0.25 & \\
\hline$v_{u}$ & Undrained P. ratio & 0.337 & \\
\hline$K_{s}$ & Solid bulk modulus & $4.5 \times 10^{04}$ & $\mathrm{MPa}$ \\
\hline$K_{f}$ & Fluid bulk modulus & $2.5 \times 10^{03}$ & $\mathrm{MPa}$ \\
\hline$c^{f}$ & Fluid diffusivity & $6.16 \times 10^{-05}$ & $\mathrm{~m}^{2} / \mathrm{s}$ \\
\hline$\phi_{0}$ & Porosity & 0.01 & \\
\hline$k$ & Dynamic permeability & $4.053 \times 10^{-07}$ & darcy \\
\hline$B$ & Skempton's coefficient & 0.815 & \\
\hline
\end{tabular}

The predicted propagation behaviours in the three different regimes were for Mode 1 loading. In this paper a partially couple model is used so that Mode 1 loading is elastic and time independent. The crack poroelastic response is distinguished by he effects induced by Mode 2 loading (pore pressure). Adopting a similar approach, however, one can identify 3 regimes of crack propagation corresponding to different degrees of pore pressure diffusion into the rock. In the fast propagation regime, the pore pressure effect on the fracture growth is negligible and the rock can be regarded as an elastic medium. In the intermediate regime, the pore pressure diffusion has some impact on the propagation behavior. And in the slow regime, the crack propagates at such a low speed that the poroelastic effect on the fracture propagation can be significant. The relative crack closure can cause a possible change in the propagation mode and direction.

In this study, $c^{f}$ is on the order of $10^{-5}, L$ the order of $10^{-1}$ and $\omega$ the order of $10^{-3}$. Different crack tip propagation speeds are chosen to model the fracture propagation in three different regimes: $v=0.1 \mathrm{~m} / \mathrm{s}$ for the fast regime; $v=1.0 \times 10^{-3} \mathrm{~m} / \mathrm{s}$ for the intermediate regime and $v=1.0 \times 10^{-5} \mathrm{~m} / \mathrm{s}$ for the slow regime.

The crack propagation path at different crack propagation speed when $\mathrm{S}_{\mathrm{H}}=1 \mathrm{MPa}, \mathrm{S}_{\mathrm{h}}=0.5$ $\mathrm{MPa}, \mathrm{P}=2.5 \mathrm{MPa}, \mathrm{c} / \sigma_{t}=1.5$ is plotted in Figure 5.9. It can be observed from the figure that when the crack propagates in an intermediate or fast regime, the propagation path is very close to that calculated by the elastic solution. It is because that the crack propagates in Mode I, i.e., opening propagation mode in this example. The pore pressure diffusion will cause small amount of relative crack closure, which is negligible in this case. The pore pressure diffusion decreases the tangential stress and therefore the normal driving force ahead of the crack tip by nearly the same degree in all directions, so the crack propagation path, which is determined by the direction of maximum normal driving force in Mode I propagation, has not been changed. However, when the crack propagates in the slow regime, the pore pressure diffusion causes the normal driving force ahead of the crack tip to drop below the microscopic tensile strength of the rock material, the propagation process therefore cannot be initiated. Another explanation of this 
phenomenon can be made that the pore pressure diffusion into the rock at the crack tip causes the decrease of crack aperture, so the crack is not able to propagate forward in Mode I - opening mode.

Crack Propagation Path at Different Propagation Speed

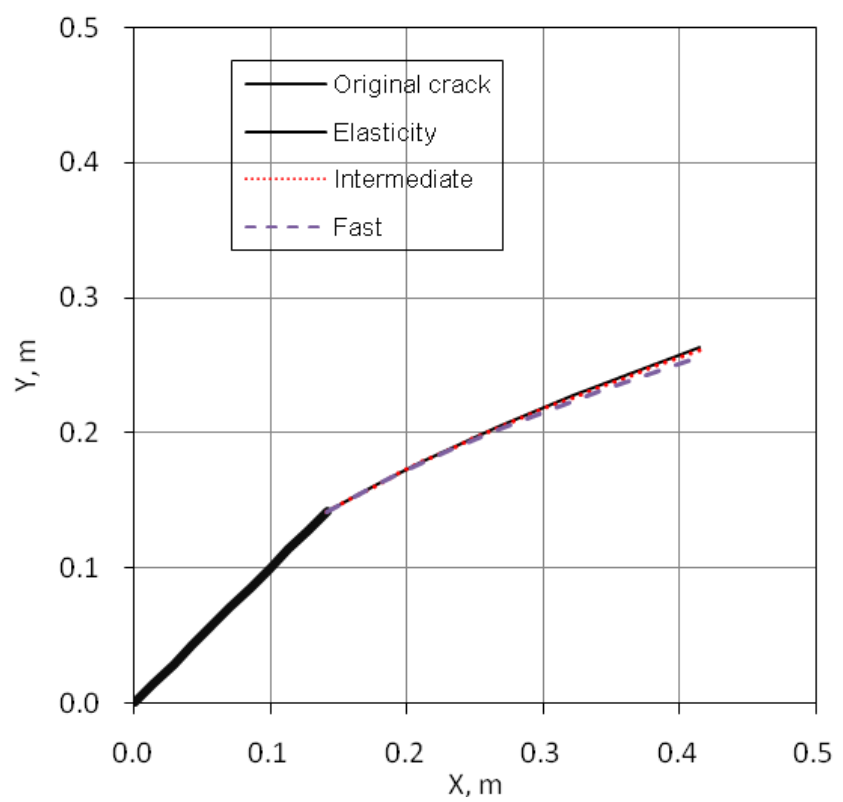

Figure 5.9. Crack propagation path at different speeds $\left(\mathrm{S}_{\mathrm{H}}=1 \mathrm{MPa}, \mathrm{S}_{\mathrm{h}}=0.5 \mathrm{MPa}, \mathrm{P}=2.5 \mathrm{MPa}\right.$, $\left.\mathrm{c} / \sigma_{t}=1.5\right)$.

The crack propagation path calculated by poroelastic model at different crack propagation speed when $\mathrm{S}_{\mathrm{H}}=3 \mathrm{MPa}, \mathrm{S}_{\mathrm{h}}=0.5 \mathrm{MPa}, \mathrm{P}=2.5 \mathrm{MPa}, c / \sigma_{t}=1.5$ is plotted in Figure 5.10 and is compared with that given by the elastic solution. As is shown in Figure 5.10, the elasticity model predicts that the crack propagation path turns to the direction of maximum in-situ stress more quickly than the last case due to the bigger difference between the maximum and minimum in-situ stress. When the crack propagates in an intermediate or fast regime, it propagates in Mode I fashion, and the propagation path calculated by the poroelastic model is very close to that calculated by the elastic model. While for the slow crack growth, it is found that the pore pressure diffusion around the crack and its tip not only leads to the relative closure of the crack surfaces, but also increases the shear driving forces around the crack tip. The crack propagates in a shear mode during the early stages of the propagation process and exhibits a different growth path from those in the fast and intermediate regimes.

In Figure 5.11, the crack propagation paths calculated by poroelastic model at different crack propagation speed for $S_{H}=3 \mathrm{MPa}, S_{h}=0.5 \mathrm{MPa}, P=2.5 \mathrm{MPa}, c / \sigma_{t}=1.1$ are plotted and is compared with that calculated by the elastic model. As predicted by the elastic 
model, in a rock of lower cohesion, Mode II (shear) propagation is more likely to occur and can be dominant in the propagation process. For a pure elastic solution, the crack first propagates in Mode II, and eventually changes direction and propagates in Mode I in the direction of maximum in-situ stress. The crack propagation path in the fast regime resembles that given by the elastic solution. While for the intermediate and slow crack growth speed, the crack propagates in Mode II only. Therefore, for cracks propagating in a poroelastic rock with low cohesion, crack growth mode could be sensitive to the propagation speed. Poroelastic effects need to be investigated more closely in order to better interpret or predict the fracture propagation behavior in the permeable material.

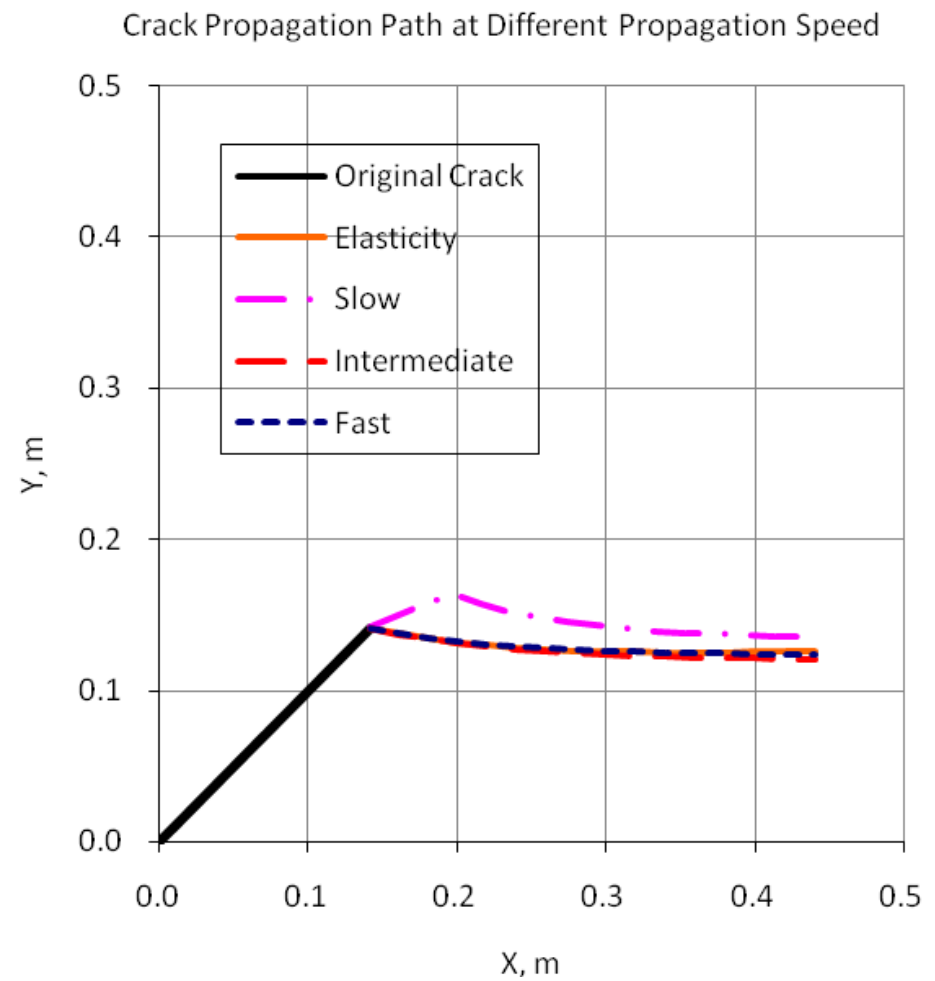

Figure 5.10. Crack propagation path at different speeds $\left(\mathrm{S}_{\mathrm{H}}=3 \mathrm{MPa}, \mathrm{S}_{\mathrm{h}}=0.5 \mathrm{MPa}, \mathrm{P}=2.5 \mathrm{MPa}\right.$, $\mathrm{c} / \sigma_{t}=1.5$ ). 
Crack Propagation Path at Different Propagation Speed

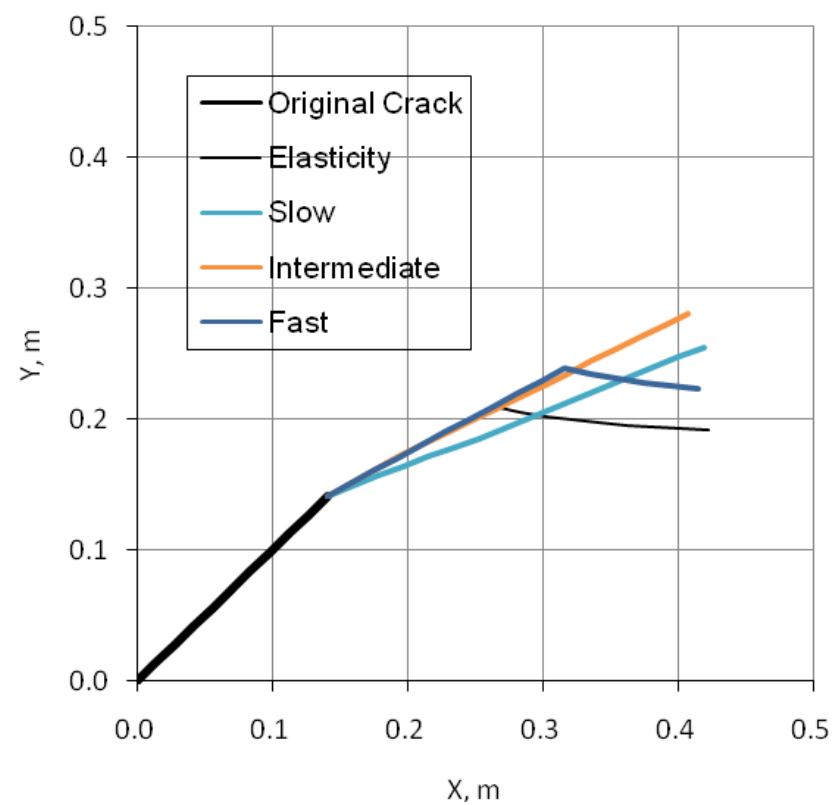

Figure 5.11. Crack propagation path at different speeds $\left(\mathrm{S}_{\mathrm{H}}=3 \mathrm{MPa}, \mathrm{S}_{\mathrm{h}}=0.5 \mathrm{MPa}, \mathrm{P}=2.5 \mathrm{MPa}\right.$,

$$
\left.\mathrm{c} / \sigma_{t}=1.1\right) \text {. }
$$

\subsubsection{Propagation of multiple pressurized cracks}

As an extension of the example shown in Figure 5.12, the propagation paths of two parallel line cracks in an infinite poroelastic medium under plane-strain conditions are investigated. For the problem under consideration, the length of each crack is $L=0.2 \mathrm{~m}$. From time $\mathrm{t}=0^{+}$, the two cracks are pressurized with a constant internal pressure $\mathrm{P}=2.5 \mathrm{MPa}$. The maximum and minimum in-situ stresses are $\mathrm{S}_{\mathrm{H}}=3 \mathrm{MPa}, \mathrm{S}_{\mathrm{h}}=0.5 \mathrm{MPa}$ respectively and $c / \sigma_{t}=1.1$. The other parameters are the same as those used in section 3.2 .

The cracks are modeled with crack propagation speed at $1.0 \times 10^{-3} \mathrm{~m} / \mathrm{s}$ in an intermediate speed regime in the poroelastic model. The poroelastic solution predicts that the two cracks will propagate in Mode II in two distinct paths. In comparison, the elastic solution predicts that Mode I will dominate the propagation process and that the left crack will coalesce with the right one in a propagation path rather different from that given by the poroelastic model. Again, pore pressure effects in this example play an important role in determining the crack propagation behaviour and interaction between multiple cracks. 


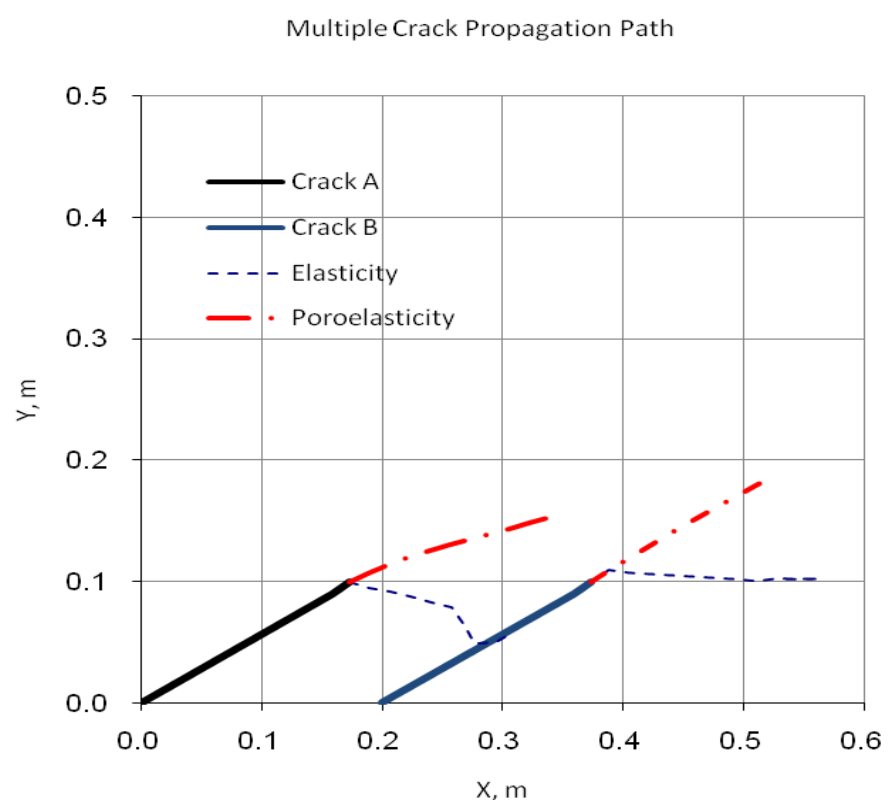

Figure 5.12. Crack propagation paths for two parallel cracks using different models $\left(\mathrm{S}_{\mathrm{H}}=3 \mathrm{MPa}\right.$,

$$
\left.\mathrm{S}_{\mathrm{h}}=0.5 \mathrm{MPa}, \mathrm{P}=2.5 \mathrm{MPa}, \mathrm{c} / \sigma_{t}=1.1\right) \text {. }
$$

Another example of crack propagation near an inclined crack in a poroelastic medium is investigated and the results are shown in Figure 5.13. Crack B is a static inclined crack, and crack $\mathrm{A}$ is allowed to propagate toward crack B. When $c / \sigma_{t}=1.1$, the fracture propagation paths are similar for crack speed of $0.1 \mathrm{~m} / \mathrm{s}$ and $1.0 \times 10^{-3} \mathrm{~m} / \mathrm{s}$, which corresponds to the fast and intermediate regime respectively. And crack propagates in Mode II in both speeds. When $c / \sigma_{t}=1.5, v=1.0 \times 10^{-3} \mathrm{~m} / \mathrm{s}$, the crack first propagates in Mode I, and then switches to Mode II before reaching crack B. While for $c / \sigma_{t}=1.5$, $v=0.1 \mathrm{~m} / \mathrm{s}$, the crack growth path is similar to that of the fast regime at the early stage of propagation process, but the pore pressure effect is more considerable and it leads to the termination of the fracture growth before the fracture reaches crack B. Therefore, poroelastic effects are important when determining the crack propagation behaviors involving interaction between multiple cracks. In these situations, moreover, rock and fluid properties, fracture geometry, in-situ stresses, internal fluid pressure and fracture propagation speed all play an important role in determining the fracture propagation behaviors. Numerical simulation by using boundary element method provides a versatile means for us to predict the fracture propagation trajectory. 


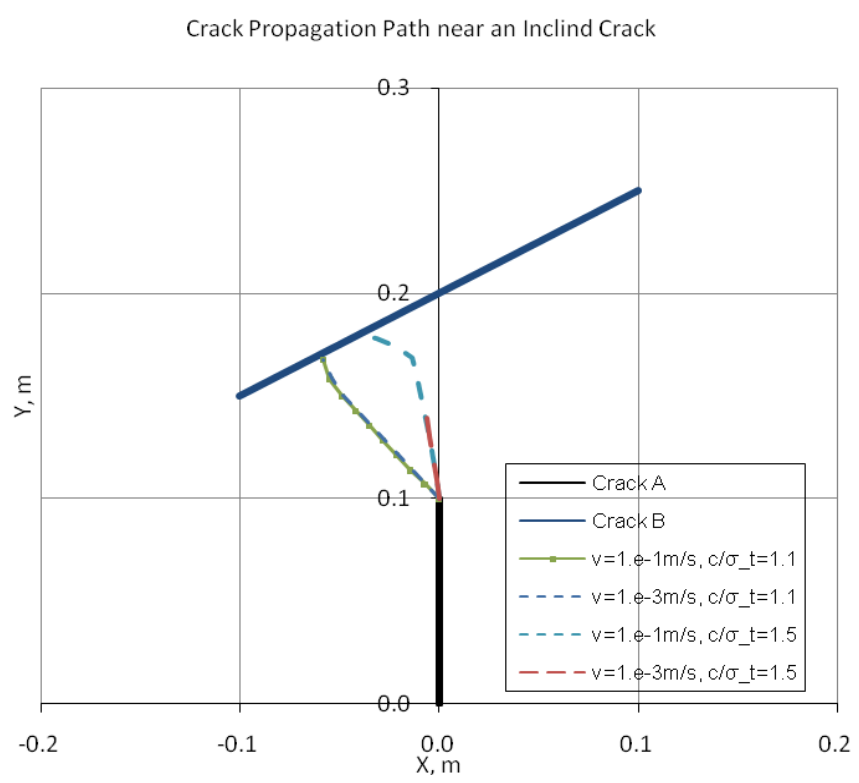

Figure 5.13. Crack propagation path near an inclined crack at different crack propagation speed $\left(\mathrm{S}_{\mathrm{H}}=1 \mathrm{MPa}, \mathrm{S}_{\mathrm{h}}=0.5 \mathrm{MPa}, \mathrm{P}=3.5 \mathrm{MPa}, \mathrm{c} / \sigma_{t}=1.1\right)$.

The results presented above illustrate the importance of the role of Mode 2 loading (pore pressure) in fracture propagation. The diffusion of pore pressure inside the crack into the rock can lead to change of possibility, mode and direction of fracture propagation. In most cases, besides fracture growth speed, rock and fluid properties, fracture geometry, in-situ stresses and internal fluid pressure all play an important role in determining the fracture propagation behavior in a permeable poroelastic medium. This numerical model can also be extended to further investigate the interaction between hydraulic fractures and natural faults in the future. A fully coupled poroelastic model can also be developed to fully describe the poroelastic effects of Mode 1 and Mode 2 loading on the fracture growth, which will enable us to have a better understanding of fracture treatments in complex geothermal environment.

\subsection{Response Injection/Extraction into a Fractured Reservoir}

The poro-thermoelatic DD boundary element method with joints (Section 3.4) has been used to develop a fractured reservoir model for simulation injection/extraction experiments. The model is applied herein to study the impact of poro-thermoelastic stresses on reservoir permeability and impedance during injection and extraction.

As an example, consider the case of a reservoir with intact rock properties of Table 2 . There is an injection well and a production well in the fractured reservoir $(2000 \times 2000$ $\mathrm{m}^{2}$ ) that has two sets of joints with an angle of $60^{\circ}$. The reservoir geometry is two- 
dimensional (horizontal section) and is suitable for situation where the natural fractures are steeply dipping. Both the injection and production rate are 1 liters $/ \mathrm{sec} / \mathrm{m}$-thickness (10 $\mathrm{m}$ thick zone). The initial reservoir pressure and temperature are $27 \mathrm{MPa}$ and $420 \mathrm{~K}$, respectively. The injectate temperature is $300 \mathrm{~K}$ and initial fracture aperture is $1 \mathrm{~mm}$. The simulation uses a constant stiffness for the joints so that the pressure change and fracture aperture change are independent of the absolute in-situ stresses, thus the observations are valid for any appropriate stress field. Also, in this example, it is assumed that shear dilation is suppressed. Other parameters are listed in Table 2.

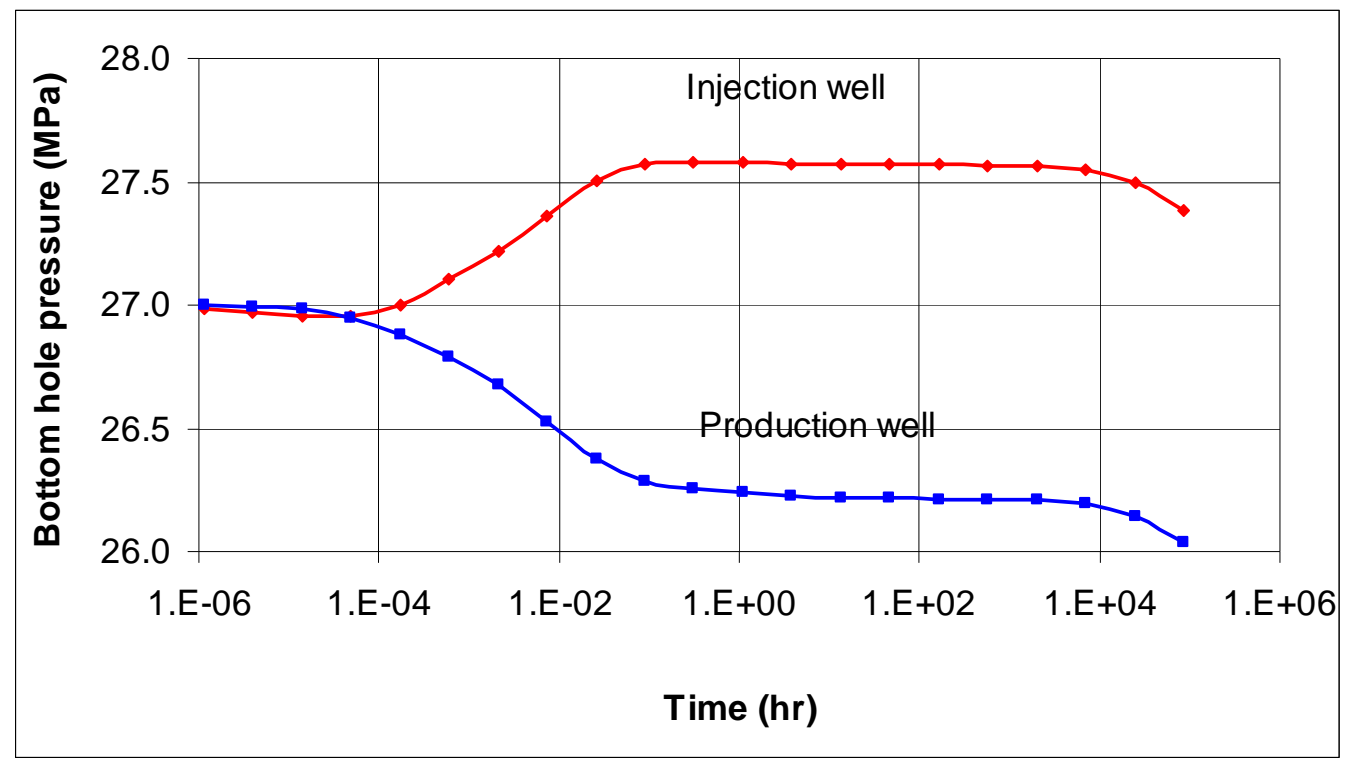

Figure 5.14. Bottomhole pressure variation with cooling (semilog).

The pressure at the injection and extraction wells is shown in Figure 5.14 as a function of time. As the injection water is cooler than the reservoir, it will cause the reservoir to shrink and reduce the reservoir pressure. However, note that for the injection well, the pressure continues to increase for some time, this caused by poroleastic effects that tend to reduce fracture aperture. The pressure distribution in the fracture network is shown in the following figures for 1 day, 1 week, 1 month, and 1 year of operation, respectively. 


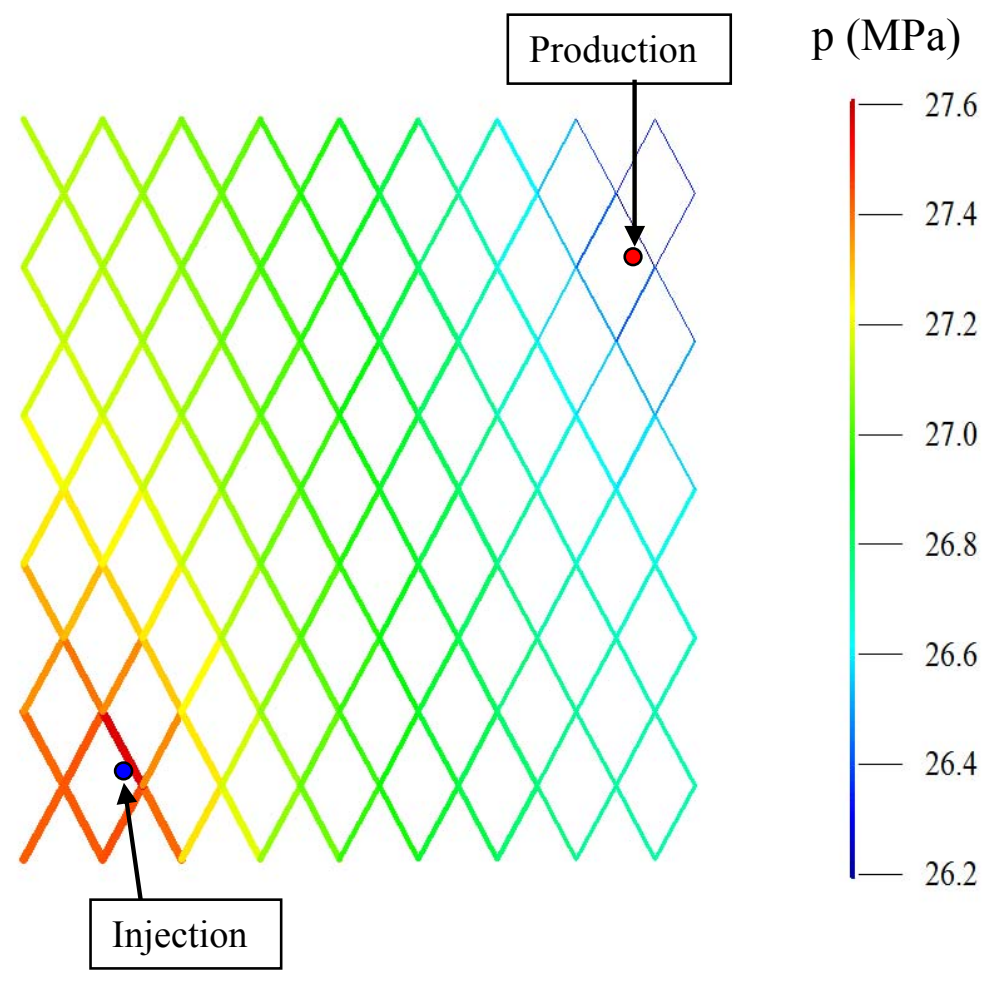

Figure 5.15. Reservoir pressure distribution with cooling effect at one day.

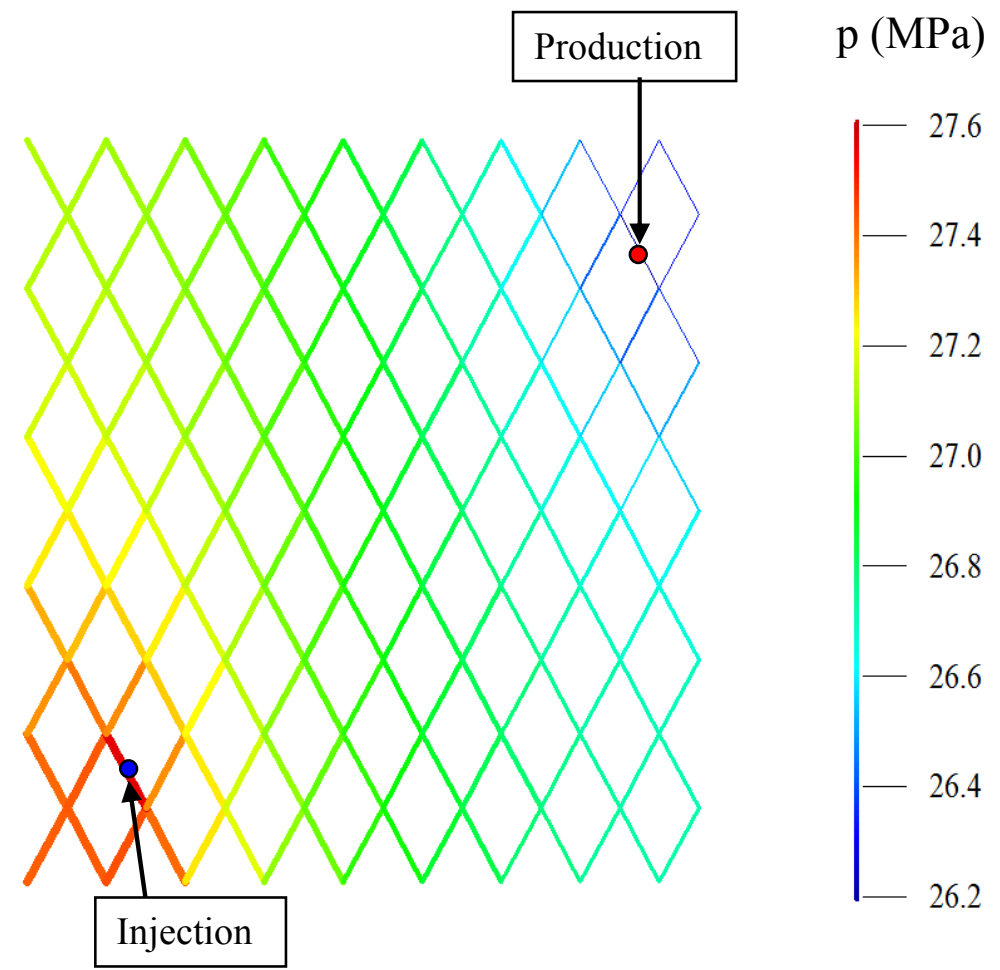

Figure 5.16. Reservoir pressure distribution with cooling effect at one week. 


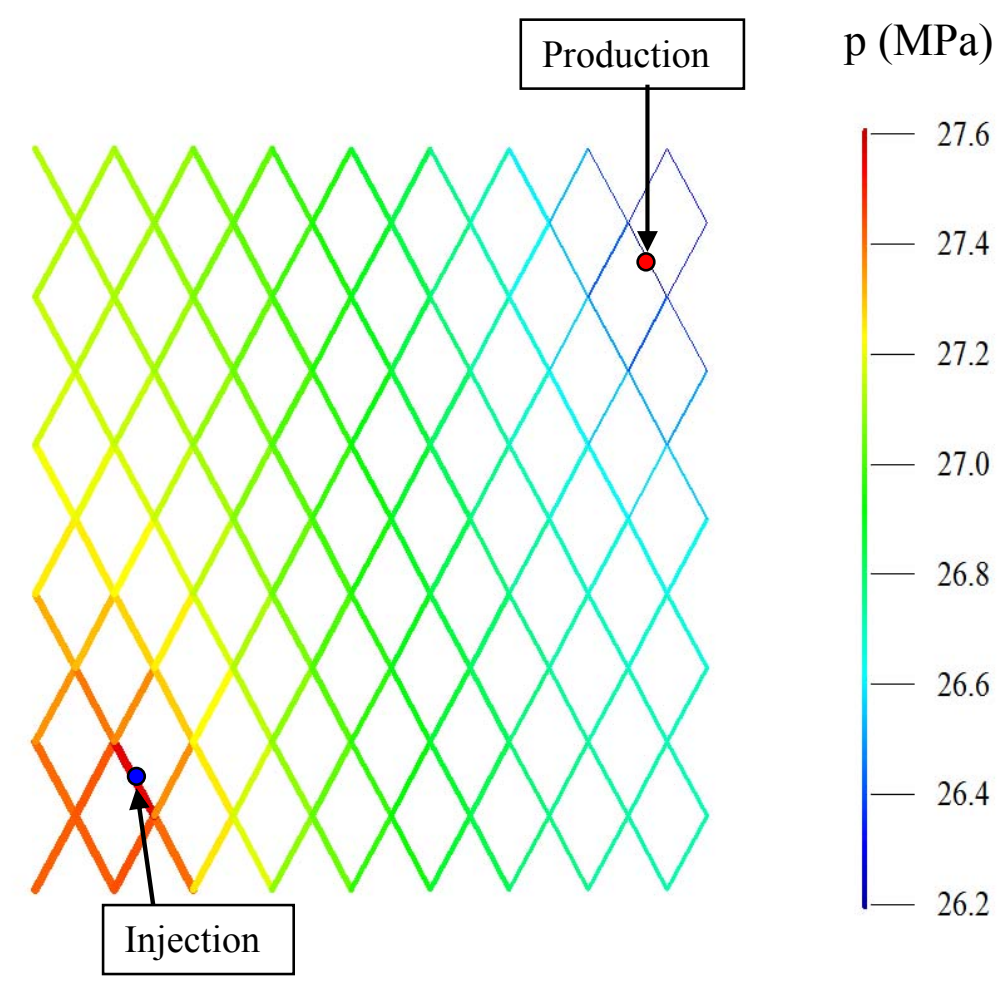

Figure 5.17. Reservoir pressure distribution with cooling effect at one month.

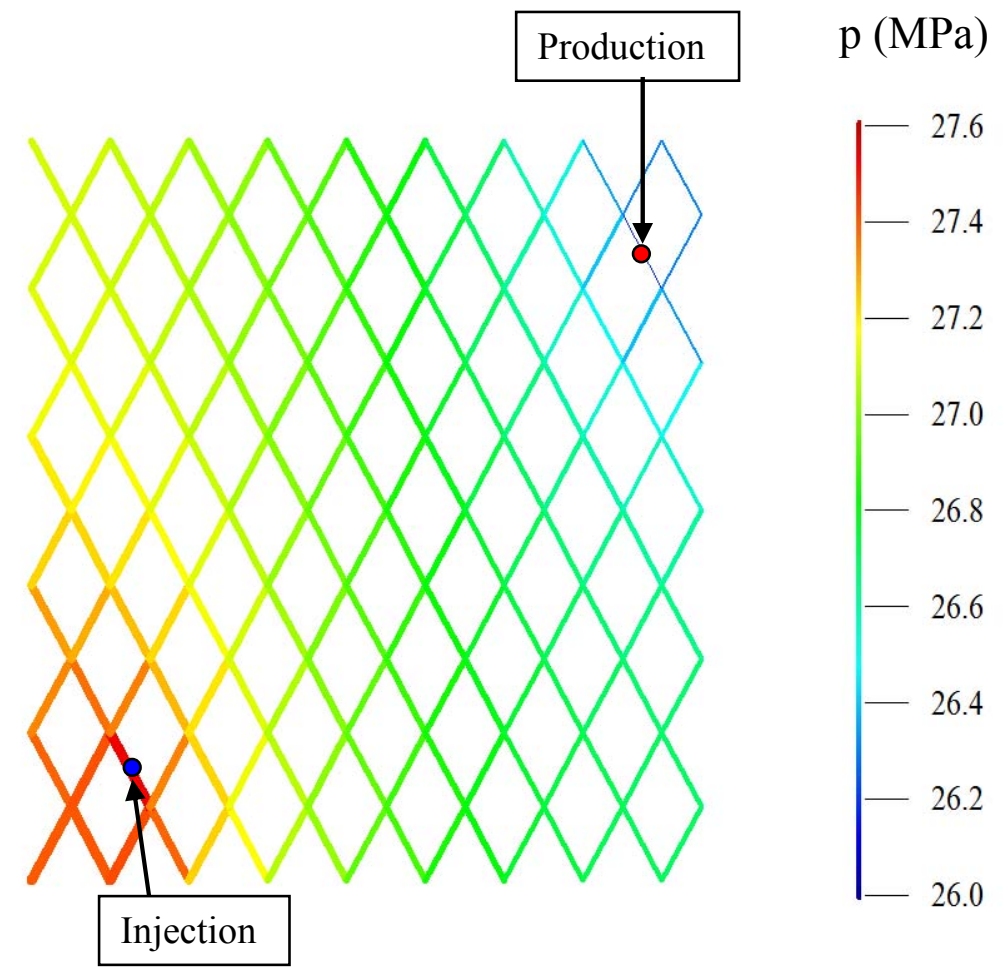

Figure 5.18. Reservoir pressure distribution with cooling effect at one year. 


\subsection{Three-Dimensional Poroelastic Analysis of Natural Fractures}

\subsubsection{Model verification}

In this section we present a number of examples dealing with fracture slip and permeability change using a 3D model. The model elements were described in Chapter 4. To verify the numerical model, we compare its predictions with the available analytical solutions for the penny-shaped crack problem. Sneddon [2] solved the problem of a crack in infinite space, subjected to uniform normal traction $p$ (see Table 3 for material properties used).

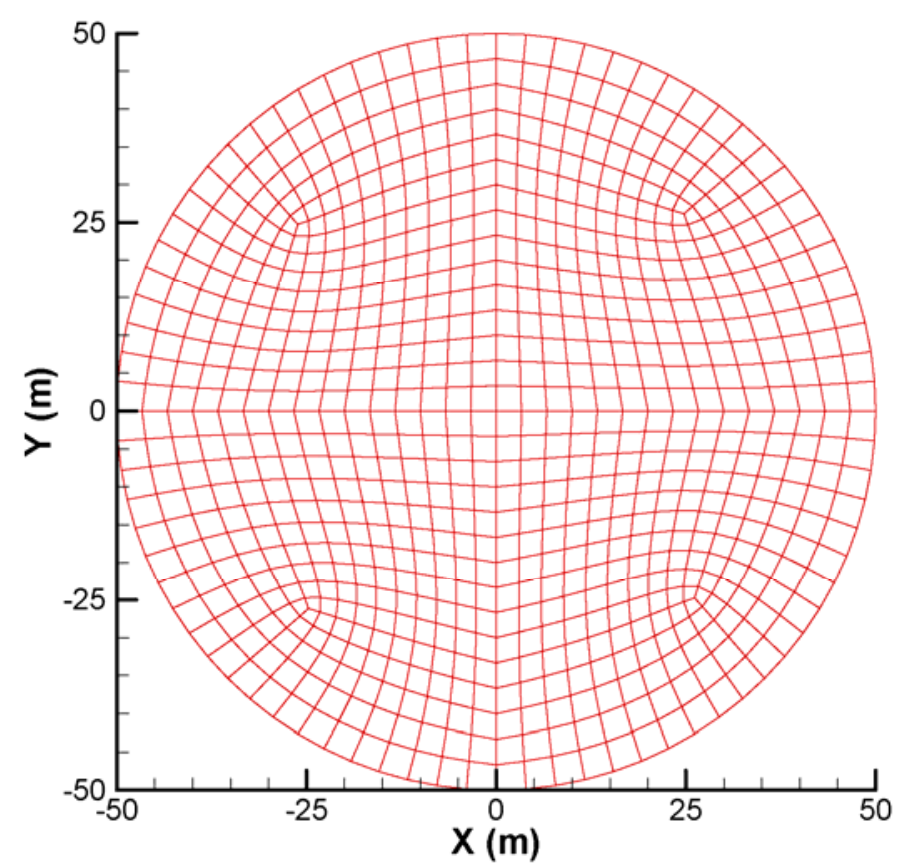

Figure 5.19. Mesh for a circular fracture used to verify the 3D numerical model.

The fracture opening in the normal direction is given by:

$$
w(r)=\frac{4(1-v) p a}{\pi G} \sqrt{1-(r / a)^{2}}
$$

where $a$ is the radius of the fracture, $r$ is the radius of the computational point, $G$ is the shear modulus, and $v$ is the Poisson's ratio. Also, Segedin [7] solved the problem of an infinitely thin penny-shaped fracture whose faces are subjected to uniform shearing tractions,$S$. The ride of the fracture in the direction of the shear force is given by:

$$
u(r)=\frac{8(1-v) S a}{\pi G(2-v)} \sqrt{1-(r / a)^{2}}
$$


Table 3. Data set used in the numerical example.

\begin{tabular}{|l|l|}
\hline Parameter & Value \\
\hline Shear modulus $G(\mathrm{GPa})$ & 4.0 \\
\hline Poisson's ratio $v$ & 0.25 \\
\hline Fluid viscosity $\mu_{f}\left(\mathrm{~N} . \mathrm{s} / \mathrm{m}^{2}\right)$ & 0.001 \\
\hline Fluid diffusivity $c_{f}\left(\mathrm{~m}^{2} / \mathrm{s}\right)$ & $10^{-3}$ \\
\hline Biot's coefficient $\alpha$ & 0.95 \\
\hline Fluid density $\rho_{f}\left(\mathrm{~kg} / \mathrm{m}^{3}\right)$ & 1000 \\
\hline Rock density $\rho_{r}\left(\mathrm{~kg} / \mathrm{m}^{3}\right)$ & 2650 \\
\hline Rock permeability $\kappa\left(\mathrm{m}^{2}\right)$ & $10^{-16}$ \\
\hline
\end{tabular}

As the material is poroelastic, the fracture aperture is time dependent. So, to compare our results with analytical solutions, we use the numerical solution at very large time (here we use $10^{8} \mathrm{~s}$ for a rock with $10^{-15} \mathrm{~m}^{2}$ of permeability) to represent the drained behavior. We set the shear modulus and Poisson's ratio of the material to $4000 \mathrm{MPa}$ and 0.25 , respectively. Figure 5.20 shows the fracture mesh with 800 four-noded quadrilateral elements and 841 nodes. The size of typical elements is around $1.5 \times 1.5 \mathrm{~m}^{2}$ and the time increment is $10^{6} \mathrm{~s}$ in the computation. Figure 5.20(a) shows the comparisons between the numerical and analytical solutions for the opening of the fracture under a unit uniform normal traction. The results for the fracture ride under a unit uniform shear tractions applied at the fracture surface are shown in Figure 5.20(b). Generally the numerical results agree well with the analytical results. The error of the numerical results increases near the fracture tip; this is caused by the use of constant elements instead of special tip elements.

\subsubsection{Numerical simulations}

As a first numerical experiment, consider a horizontal circular planar fracture in a poroelastic rock (Figure 5.21). The fracture is suddenly subject to a constant fluid pressure $p=15 \mathrm{MPa}$ at time $t=0$. It is assumed that the initial stresses in the field are isotropic and the vertical and horizontal components are $30 \mathrm{MPa}$ and $20 \mathrm{MPa}$, respectively. The fracture normal stiffness modulus of the fracture is assumed to be $10^{8}$ $\mathrm{Pa} / \mathrm{m}$. The problem can be decomposed into two subproblems corresponding to two types of the loadings [11]: Mode 1, a normal stress loading $\sigma_{n}=p H(t)$; and Mode 2, a pore pressure loading $p=p H(t)$, where as before $H(t)$ denotes the Heaviside step function. 


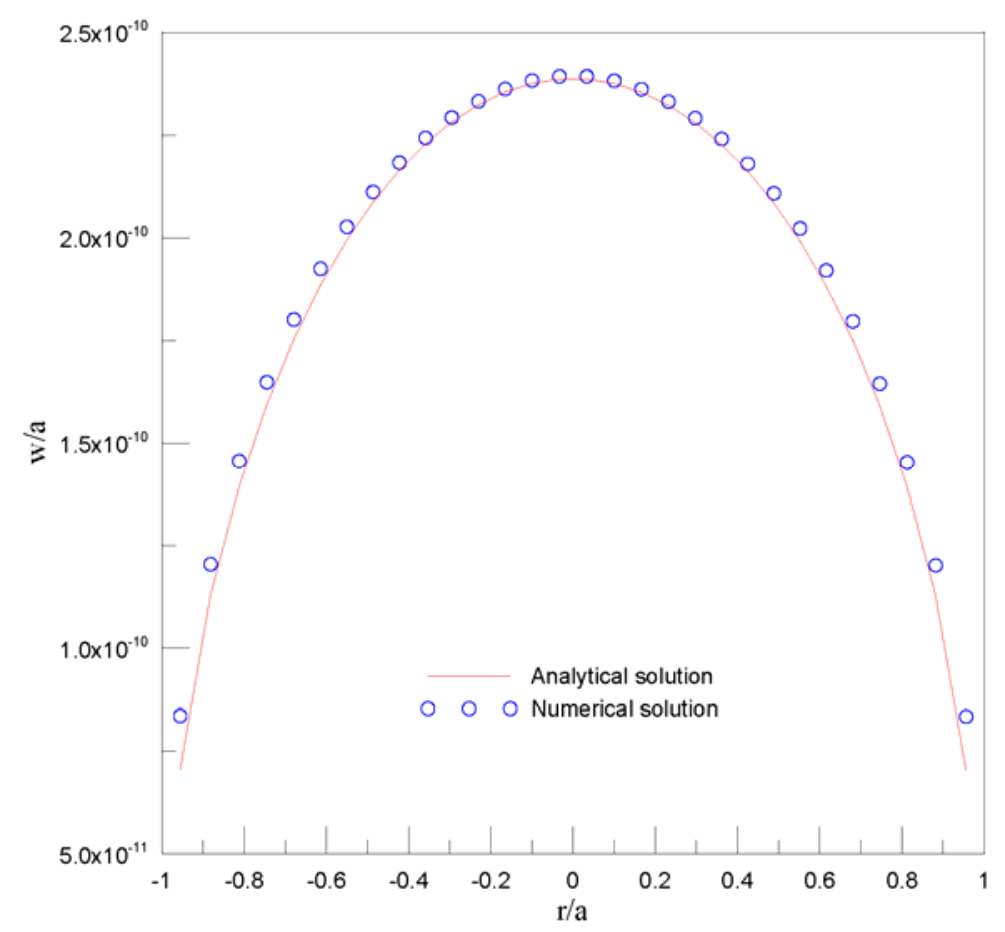

(a) normal direction

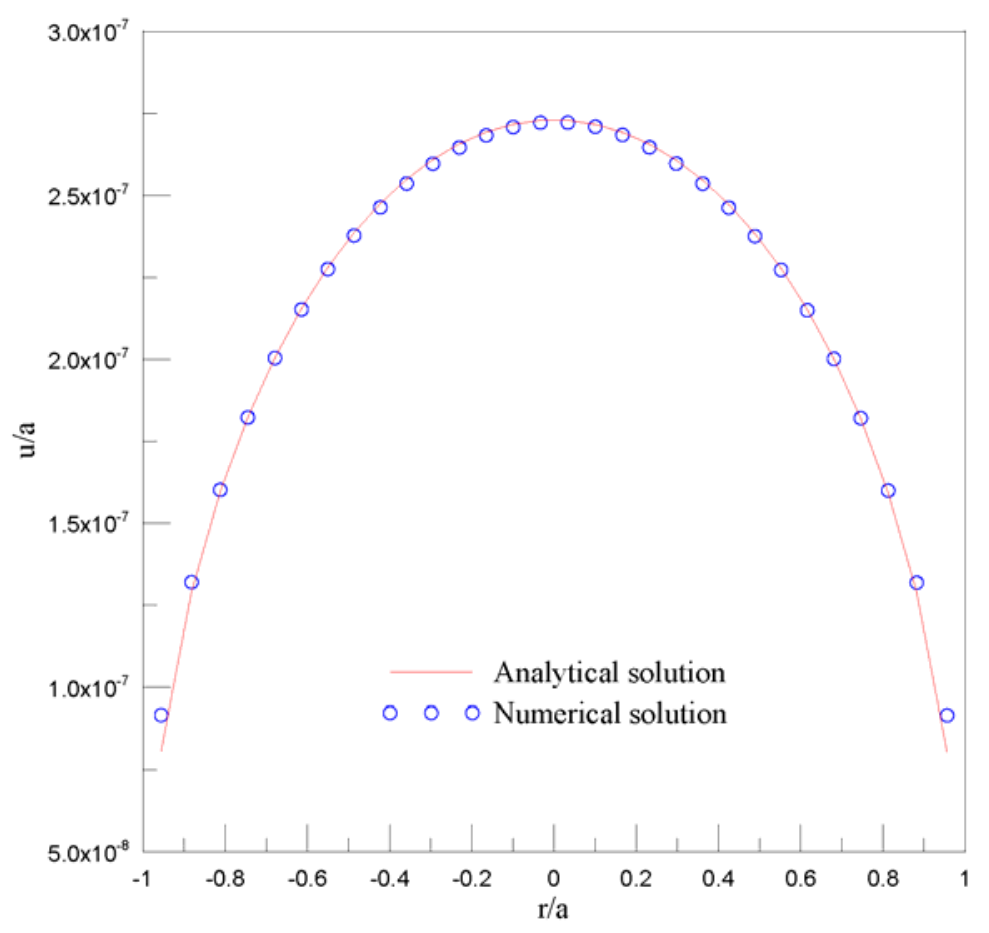

(b) shear direction

Figure 5.20. Comparisons between numerical results and analytical results in the normal and shear directions. 


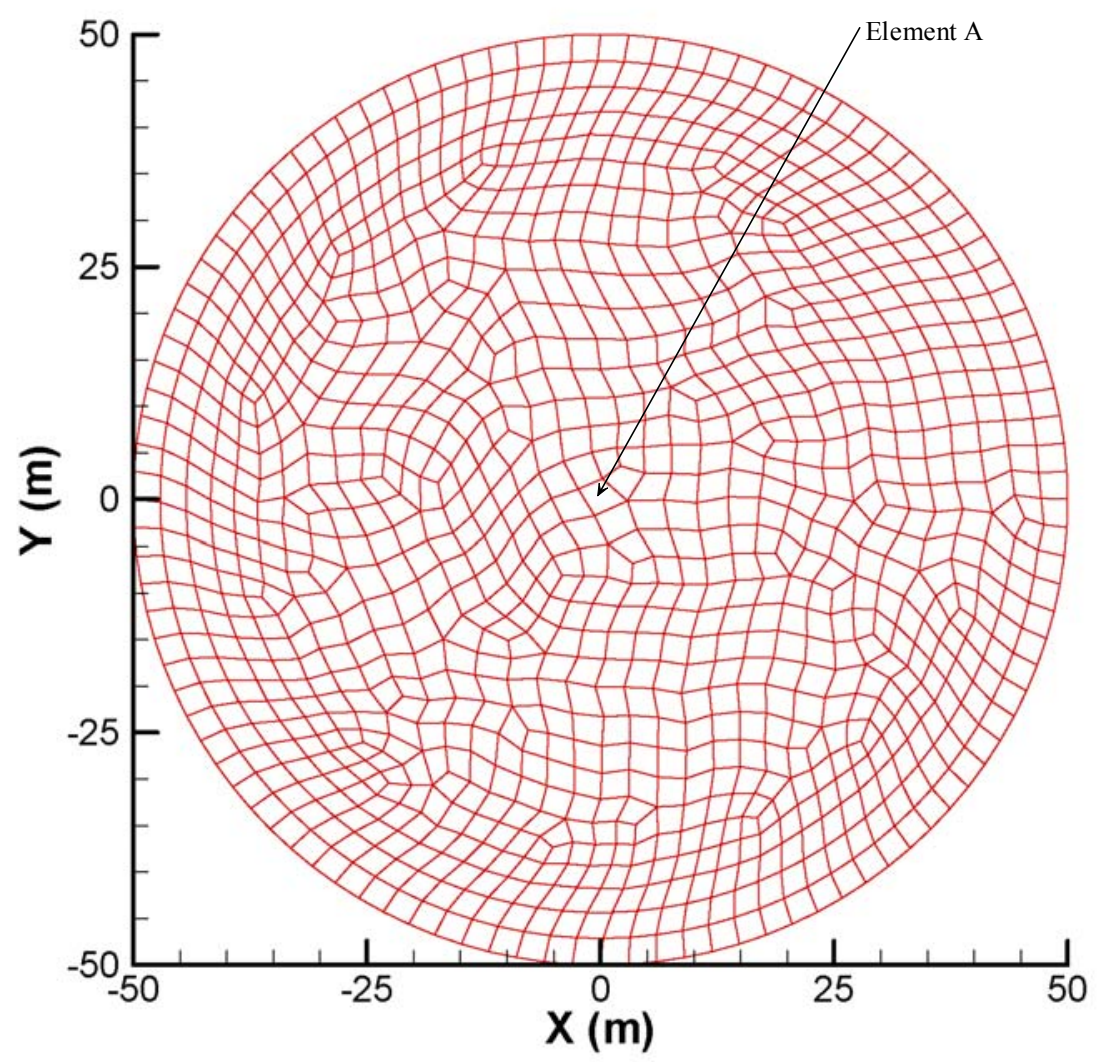

Figure 5.21. Circular fracture mesh used in numerical example, where element $\mathrm{A}$ is at the center of the fracture. 


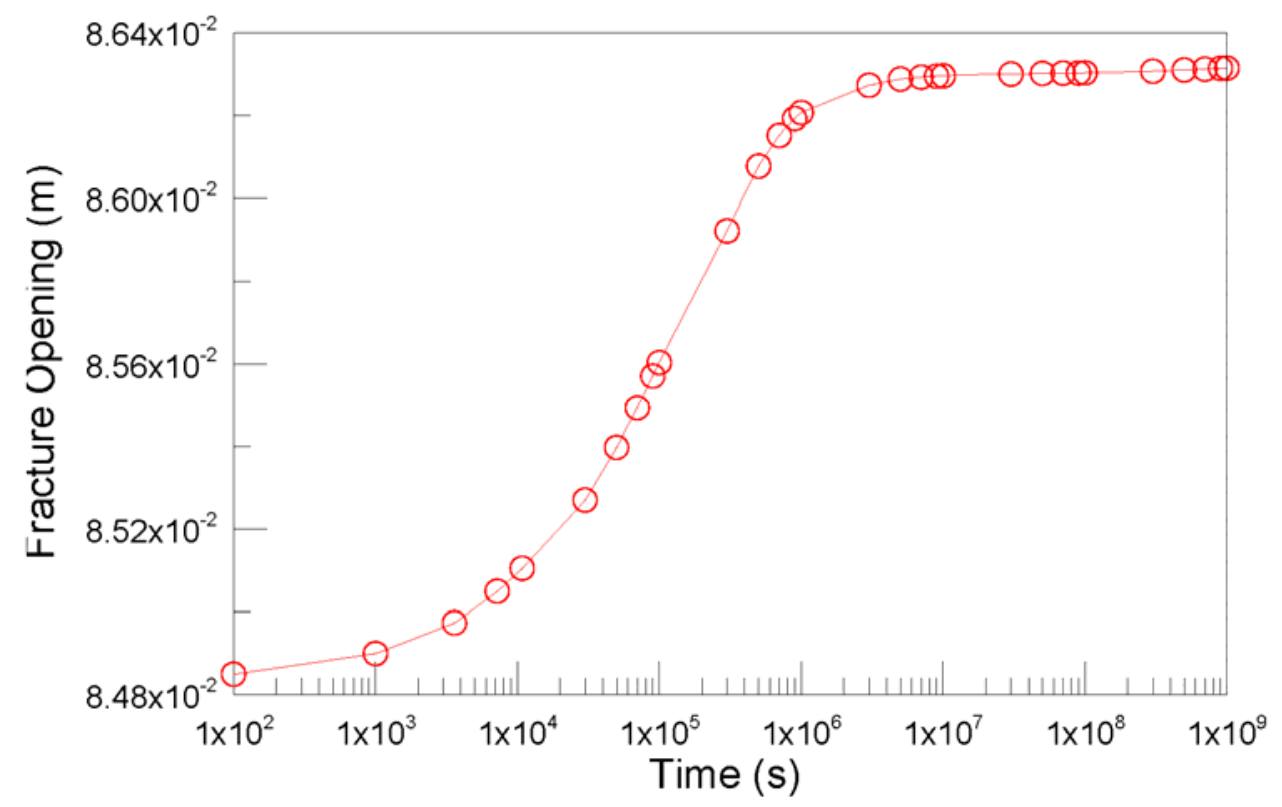

(a) Normal stress loading (Mode 1)

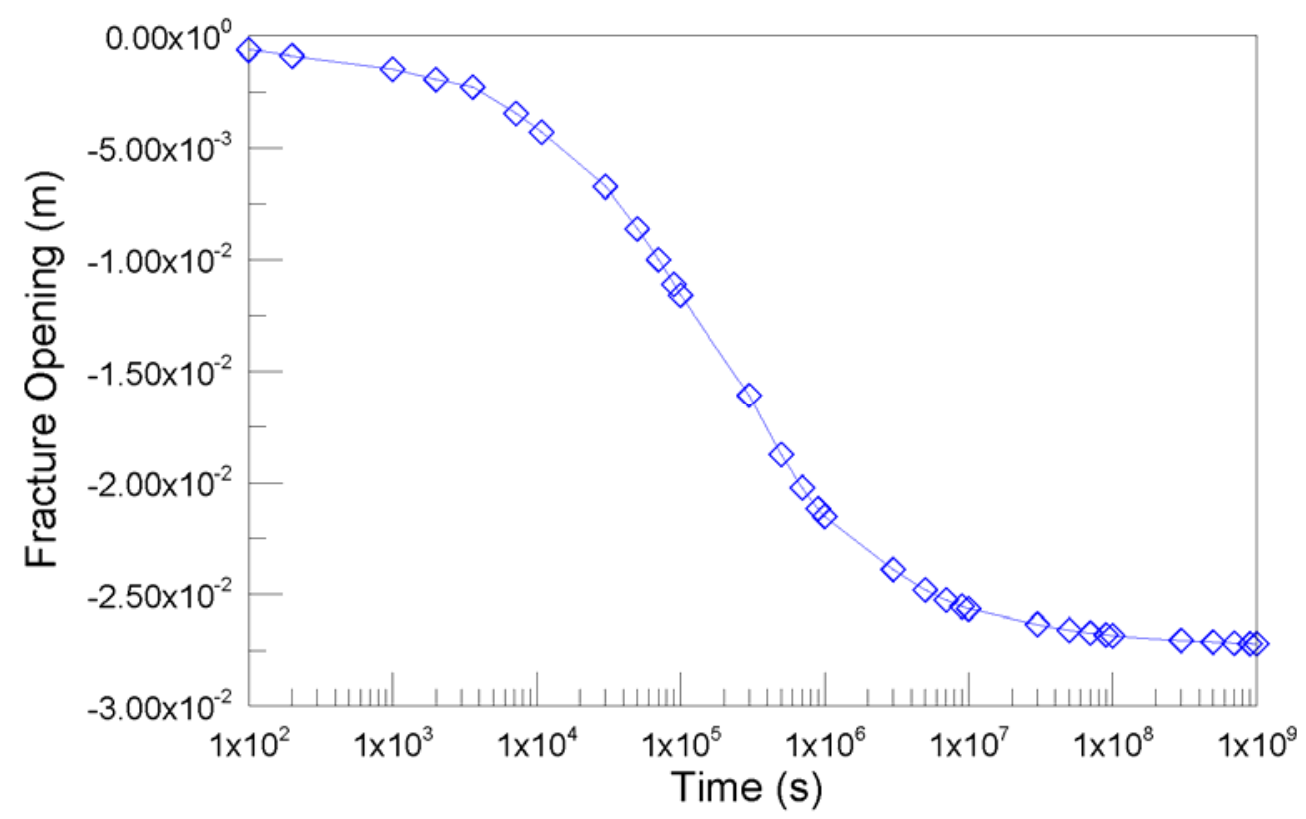

(b) Pore pressure loading (Mode 2)

Figure 5.22. Variations of fracture opening at element A due to Mode 1 and 2 loadings. 


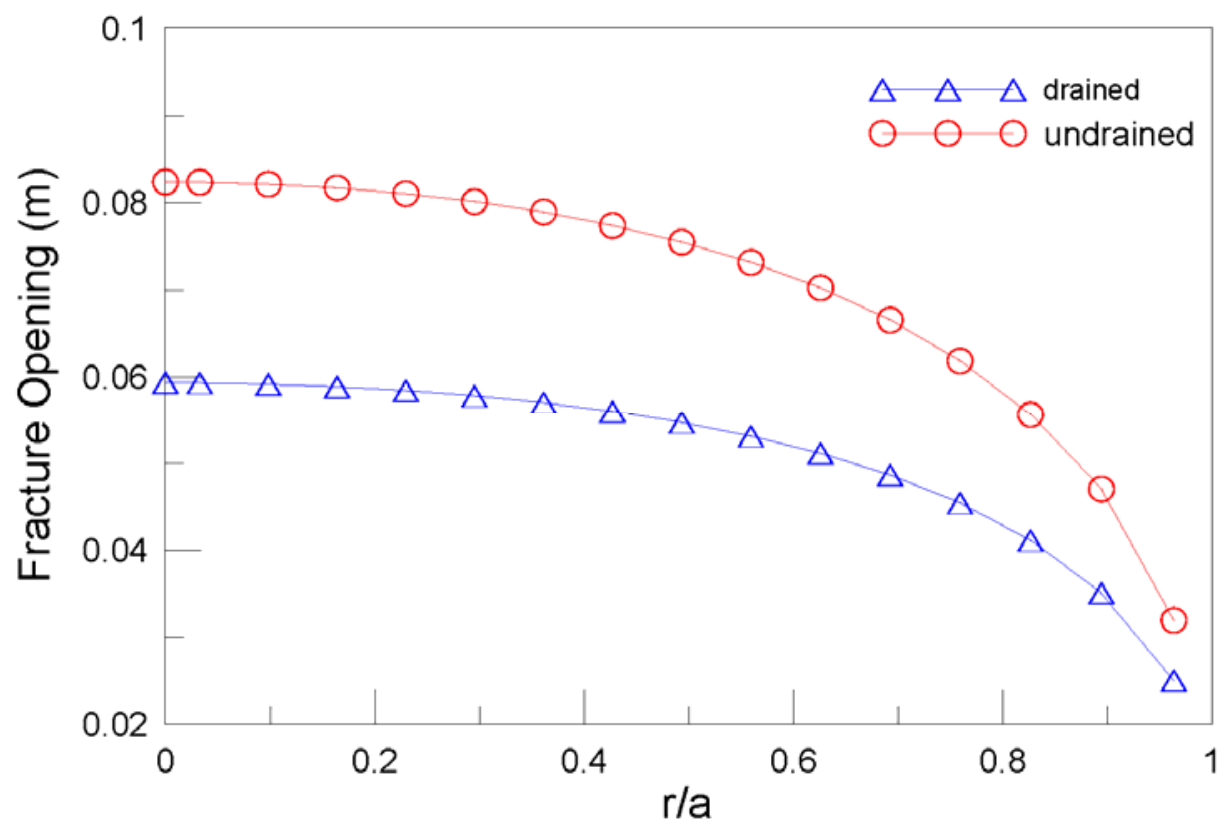

Figure 5.23. Undrained and drained fracture opening profile in response to combined mode 1 and 2 loading, where $r$ is the radius of the computational point and $a$ is fracture radius.

Figure 5.21 shows the fracture mesh used, it contains 1047 four-noded quadrilateral element and 1100 element nodes. Figure 5.22(a) shows the evolution of the fracture aperture at the center of element A (see Figure 5.21) in response to Mode 1 loading. As in the $2 \mathrm{D}$ case, it is found that the fracture opens with time as the pore pressure that is initially generated in the porous rock gradually dissipates. The fracture response at the center of element A under Mode 2 is illustrated in Figure 5.22(b). It is observed that the fracture closes progressively starting from zero to a stabilized value after a long time. This phenomenon is caused by the rock dilation when the fluid leaks-off from the fracture into the reservoir matrix and also has been observed previously for the $2 \mathrm{D}$ case. Figure 5.23 shows the fracture aperture profiles for the complete problem (both Modes 1 and 2) for the undrained and drained cases. In the undrained case, we let $t=100 \mathrm{~s}$ in the numerical simulation so that there is almost no pore pressure dissipation or fluid leak-off from the fracture into the rock; while in the drained case, we let $t=10^{8} \mathrm{~s}$ in order to allow both of Modes 1 and 2 transient processes to be complete. Note that the fracture aperture in the early time (undrained) case is larger than that of the large time (drained) response because of the effect of Mode 2 which induces a fracture closure.

\section{Irregularly-shaped natural fracture subjected to shear}

In the following, we analyze the opening and slip of a planar fracture that is subjected to a fluid pressure which is less than the in-situ minimum stress. This condition can be expected when stimulating geothermal reservoirs. The fracture surface has a dip angle of $60^{\circ}$ and its strike direction is parallel to the local $x$-axis. It is assumed that the fracture is in an in situ stress of $\sigma_{\mathrm{v}}=60.13 \mathrm{MPa}, \sigma_{\mathrm{hmin}}=34.81 \mathrm{MPa}, \sigma_{\mathrm{Hmax}}=50.88 \mathrm{MPa}$, and $p=17.4 \mathrm{MPa}$ [7]. The orientation of $\sigma_{\mathrm{Hmax}}$ is parallel to the fracture strike direction. This stress field can be rotated to the local fracture coordinate system to obtain $\sigma_{\mathrm{zz}}=41.1 \mathrm{MPa}, \sigma_{\mathrm{xz}}=0 \mathrm{MPa}$, and 
$\sigma_{\mathrm{yz}}=11.0 \mathrm{MPa}$. It is also assumed that the effective friction angle and dilation angle of the fracture are $30^{\circ}$ and $3^{\circ}$, respectively. Both the normal and shear stiffness of the fracture are assumed to be $10^{10} \mathrm{~Pa} / \mathrm{m}$. The other material properties used here are shown in Table 3 . The fracture is discretized into 1834 four-noded quadrilateral elements and 1919 element nodes as shown in Figure 5.24. For simplicity, we assume the fluid pressure in the fracture is constant and uniform and its value is $25 \mathrm{MPa}$.

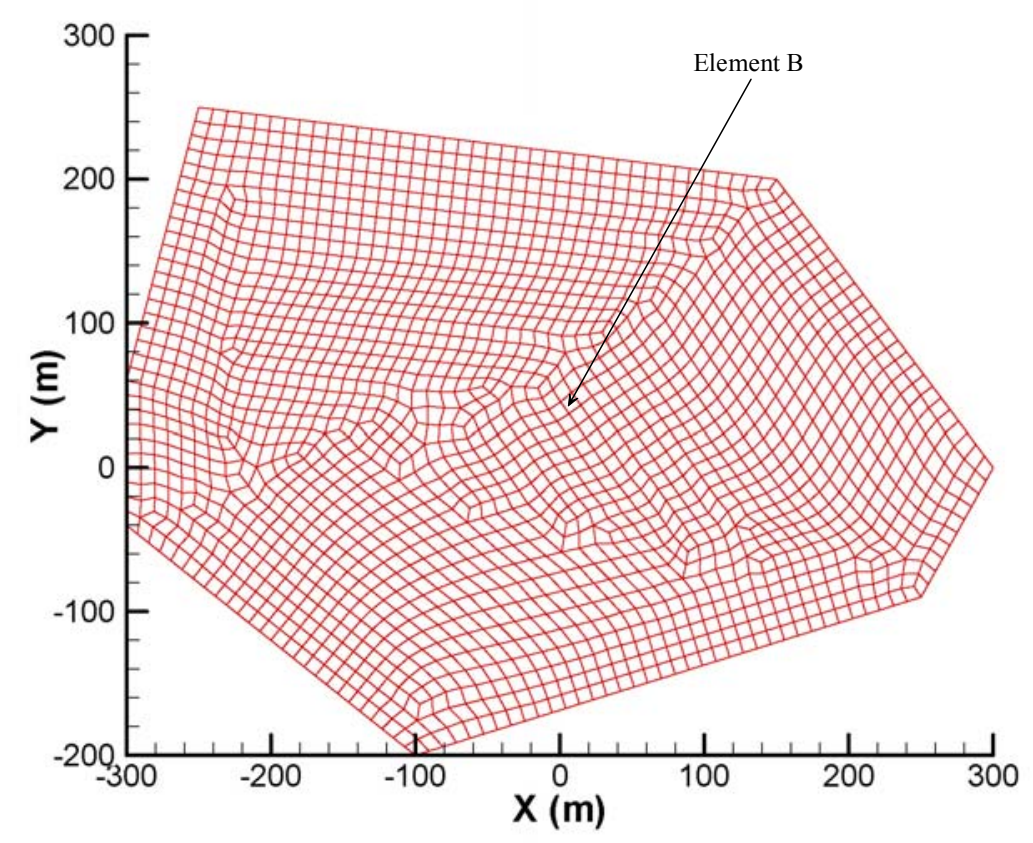

Figure 5.24. Discretization of an irregular fracture using 1834 four-noded quadrilateral elements.

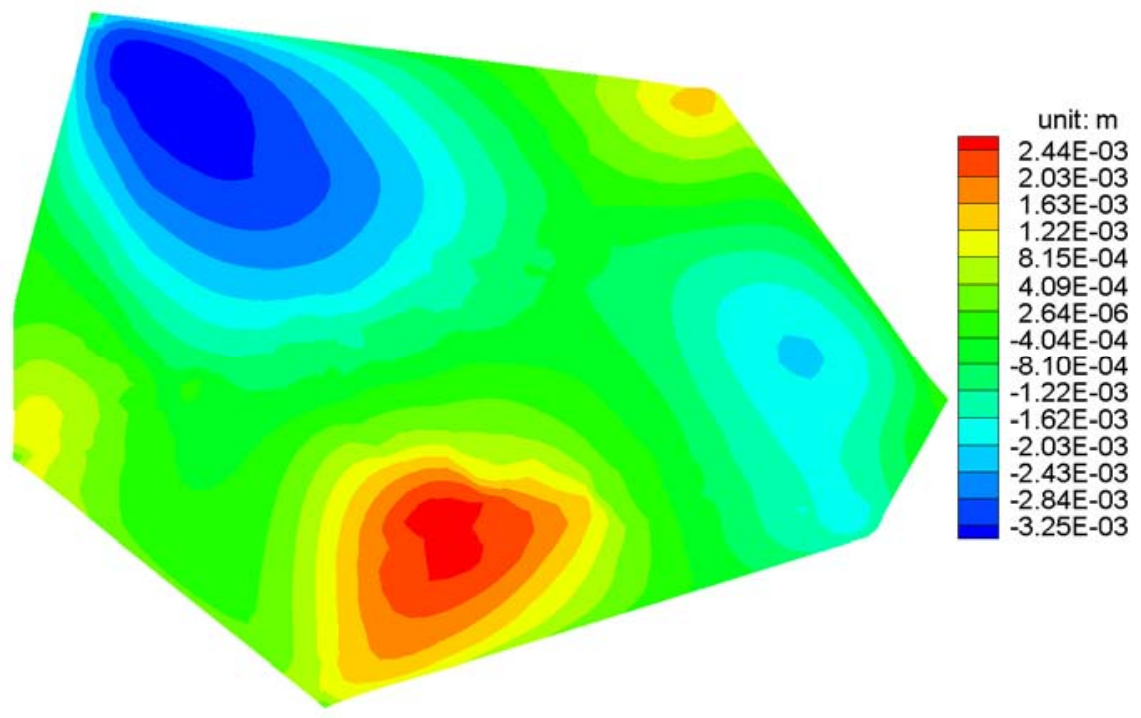

Figure 5.25. Distribution of shear displacement component $D_{\mathrm{zx}}$ on the fracture. 


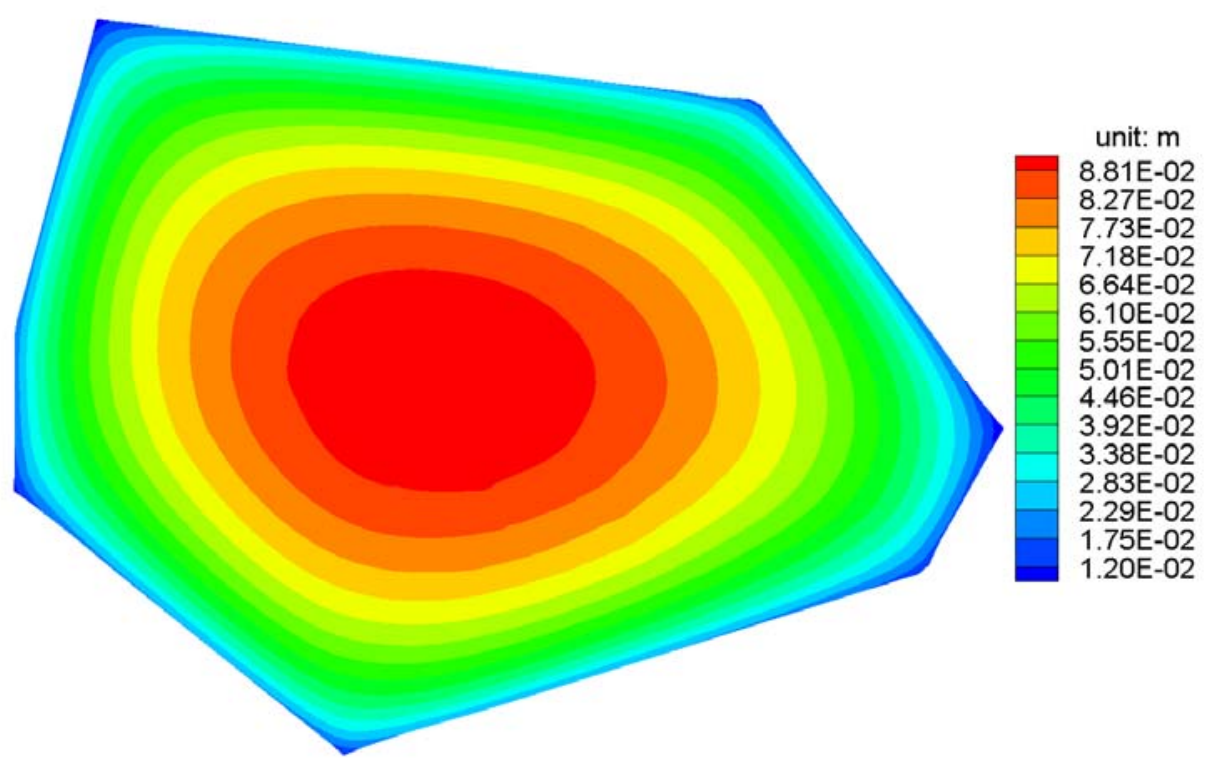

Figure 5.26. Distribution of shear displacement component $D_{\mathrm{zy}}$ on the fracture.

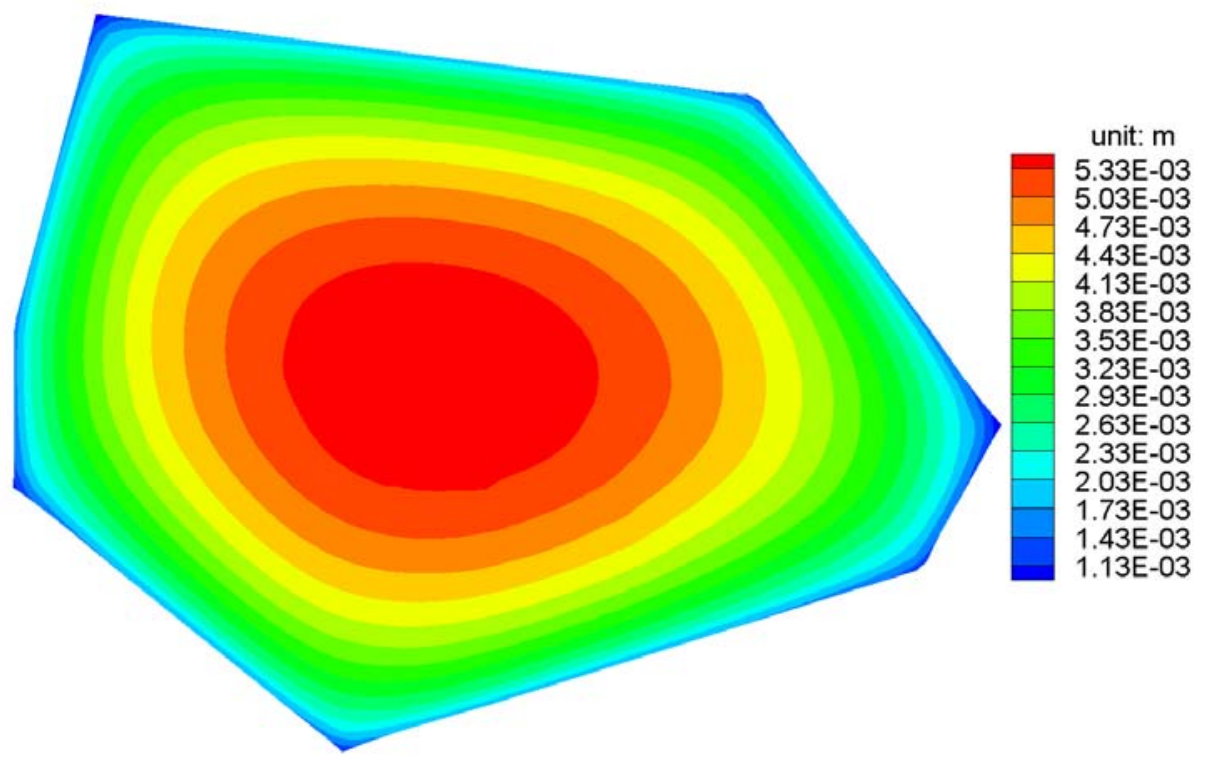

Figure 5.27. Distribution of normal displacement $D_{\mathrm{zz}}$ on the fracture. 


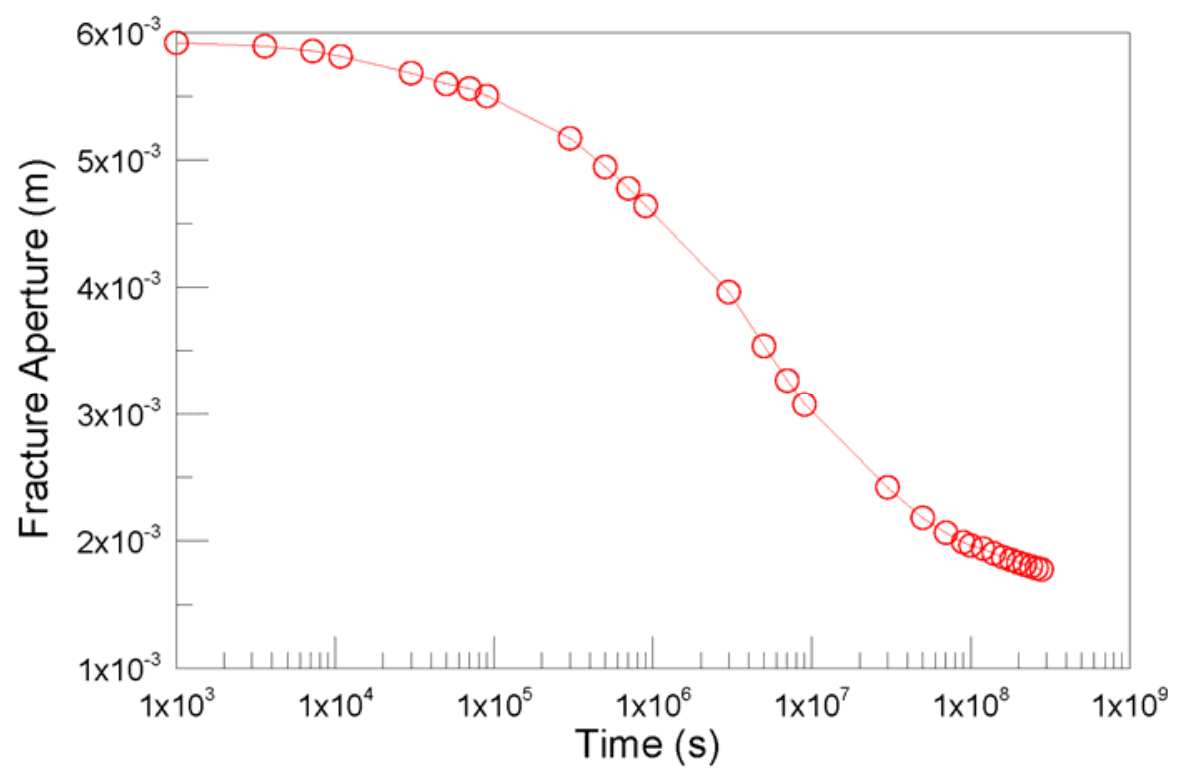

Figure 5.28. Variation of fracture aperture $d_{n}$ for element B with time.

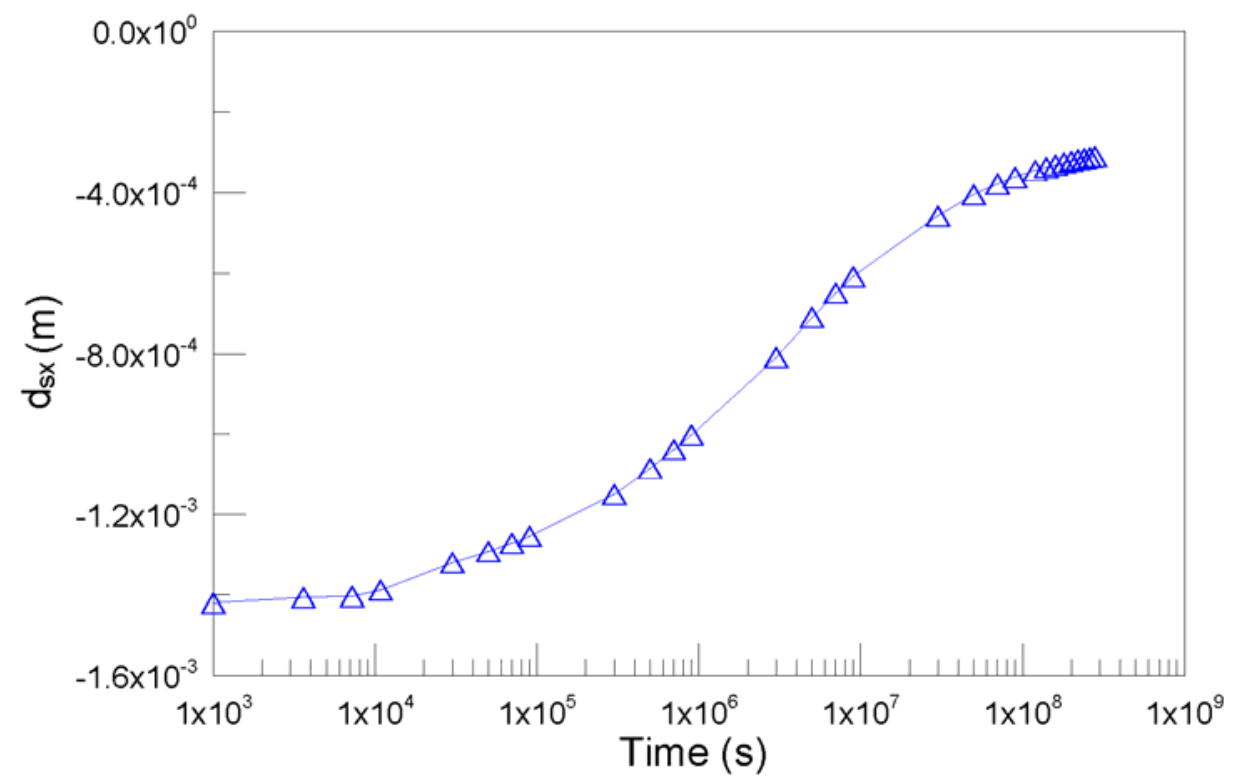

Figure 5.29. Variation of slip in X-direction $d_{s x}$ for element B with time. 


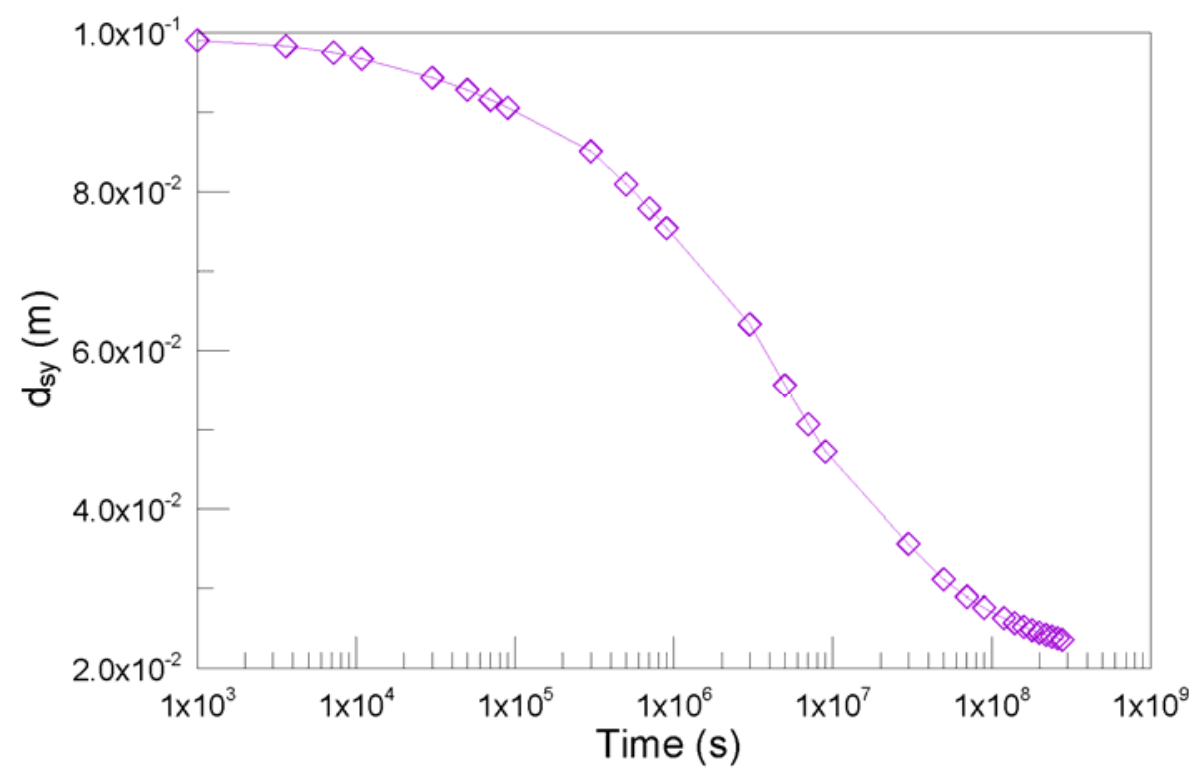

Figure 5.30. Variation of slip in y-direction $d_{s y}$ for element B with time.

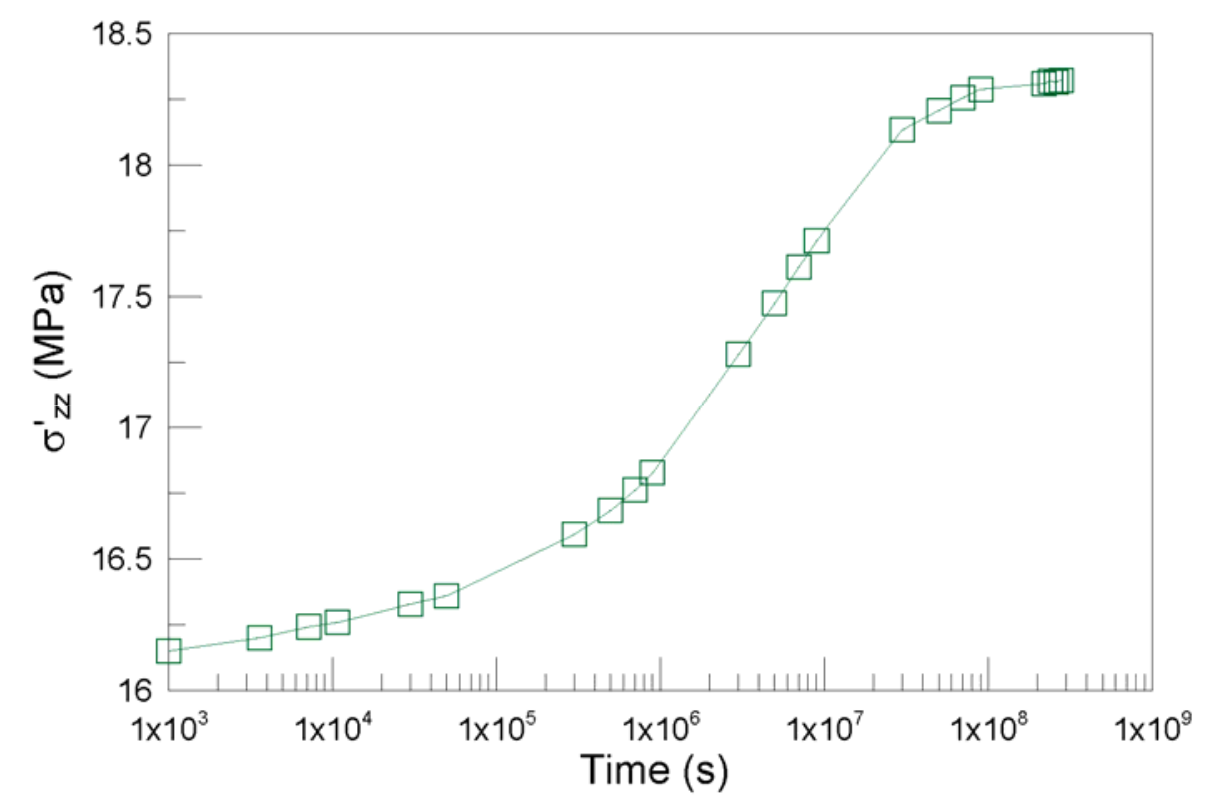

Figure 5.31. Variation of normal effective stress $\sigma_{z z}^{\prime}$ for element B with time.

Figures 5.25 and 5.26 show the distributions of the shear displacement discontinuities $D_{\mathrm{zy}}$ and $D_{\mathrm{zx}}$ in the local fracture coordinates to a time of $10^{5} \mathrm{~s}$ after the fluid pressure is applied in the fracture. As expected, it is found that $D_{\mathrm{zy}}$ is much larger than $D_{\mathrm{zx}}$ because 
the in situ shear stresses $\sigma_{\mathrm{zy}}>>\sigma_{\mathrm{zx}}$ on the fracture surface. The maximum slip in the $y$ direction occurs at the center of the fracture and equals $8.8 \mathrm{~cm}$. Figure 5.27 illustrates the distribution of the fracture opening with a maximum value of $5.3 \mathrm{~mm}$ at the center of the fracture when the time is $10^{5} \mathrm{~s}$. The increase in the fracture aperture is caused by the reduction of the normal effective stresses and shear slip induced dilations.

Because of the poroelastic nature of the rock, the fracture behavior is transient. Figures 5.28-5.31 show the variations of fracture aperture, shear slip in the $x$-direction, shear slip in the $y$-direction, and the normal effective stress on element B (see Figure 5.24). It is observed that the magnitudes of the shear slip in both the $x$-direction and $y$-direction decrease with the passage of the time. This is an interesting result made possible by our analysis; this phenomenon can be explained by the increase of the normal stresses with time. The joint shear strength is directly proportional to the normal effective stress at the fracture surface so that the fracture aperture is influenced by the variations of the normal effective stress, which changes increases in response the matrix dilation due to fluid leakoff from the fracture into the reservoir matrix and constraint dilation.

\subsection{Three-Dimensional Analysis of Natural Fracture Response to Injection and Extraction}

The combined poro- and thermoelastic model will now be applied to an injection/extraction problem to study the impact of fracture aperture change on pressure in the fracture. In this section, we present two numerical examples using the coupled model: one is for a circular fracture with one injection well and one extraction well, and the other is for an arbitrary shaped fracture with one injection well and two extraction wells.

\subsubsection{Circular fracture}

The fracture is assumed to be at a depth of $2330 \mathrm{~m}$ with an in situ stress of $\sigma_{V}=60.13 \mathrm{MPa}, \sigma_{H \text { min }}=34.81 \mathrm{MPa}, \sigma_{H \text { max }}=50.88 \mathrm{MPa}$, and a pore pressure of $p=17.4 \mathrm{MPa}$. To isolate the coupled thermal and proelastic effects, the fracture is considered as horizontal so that there is no shear slip during the fluid injection and extraction process (Figure 5.32). Initially, we consider the crack to be circular fracture with a radius of $100 \mathrm{~m}$. The water is injected at temperature $T_{i n j}$ with flow rate $Q_{i n j}$. The initial reservoir temperature is $T_{r}$. The fluid extraction pressure is assumed to be equal to the initial reservoir pressure and keeps constant in the fluid injection process. $T_{i n j}, Q_{i n j}, T_{r}$ and other parameters used in this problem are shown in Table 4. Figure 5.33 shows the mesh used here which contains 1832 four-noded quadrilateral element and 1891 element nodes. In Figure 5.33, the injection and extraction wells are located at points $\mathrm{A}$ and B, respectively. 
Figure 5.34 and 5.35 show the distributions of the vertical normal poroelastic and thermoelastic stresses, respectively, when the fluid injection times are $10^{5} \mathrm{~s}$ (1.15 days), $10^{7} \mathrm{~s}$ (3.86 months) and $10^{9} \mathrm{~s}$ (31.71 years) by using the poro-thermoelastic model. The poroelastic stresses are resulted from fluid leakoff from the fracture into the reservoir matrix; while the thermoelastic stresses are from heat exchange between the injected fluid and the reservoir matrix. The vertical poro-stresses are compressive while the thermostresses are tensile. Note that the magnitudes of the thermo-stress are much larger than those of the poro-stress, and that the thermo-stresses are tensile before the extraction well and compressive behind the extraction well.

Figure 5.36 shows the distributions of the fracture aperture for different fluid injection times. At the early time, the fracture aperture around the injection well is relatively than the other parts because the fluid pressure and the thermal stresses, both of which enlarge the fracture aperture, is larger around the injection well. Like the thermo-stresses, the fracture aperture is also relatively large before the extraction well and relatively small behind the extraction well. After very long time of the fluid injection, the apertures are larger at the zone before the extraction well and smaller at the zone behind the extraction partly because of the complex three-dimensional deformations of the fracture.

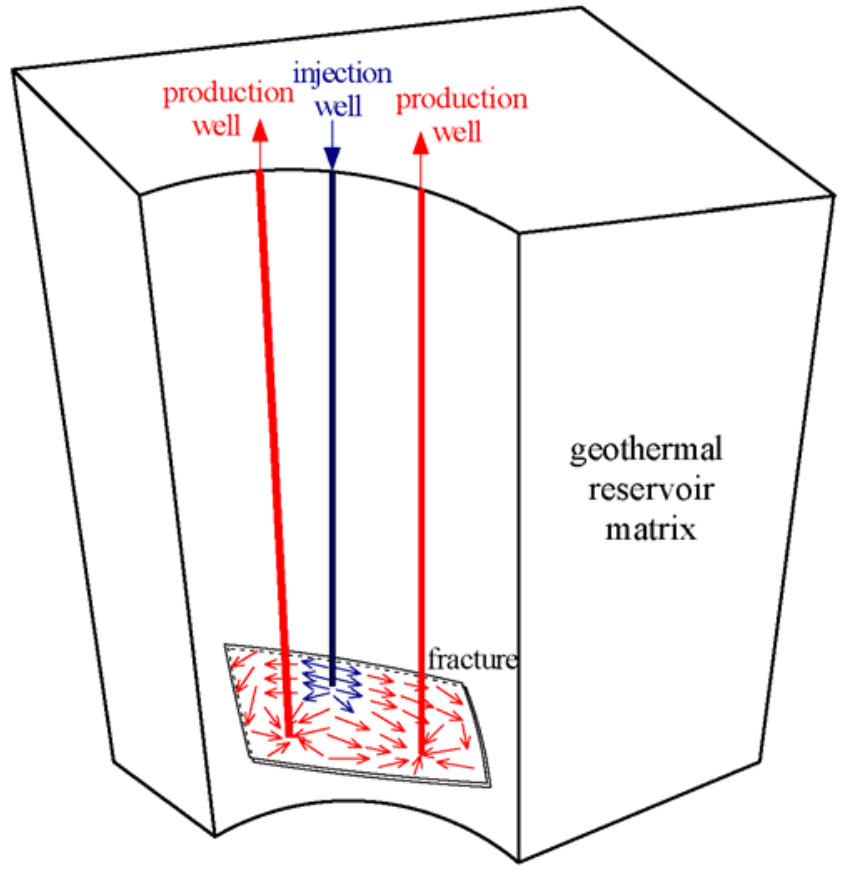

Figure 5.32. Fluid circulation in geothermal reservoir. 


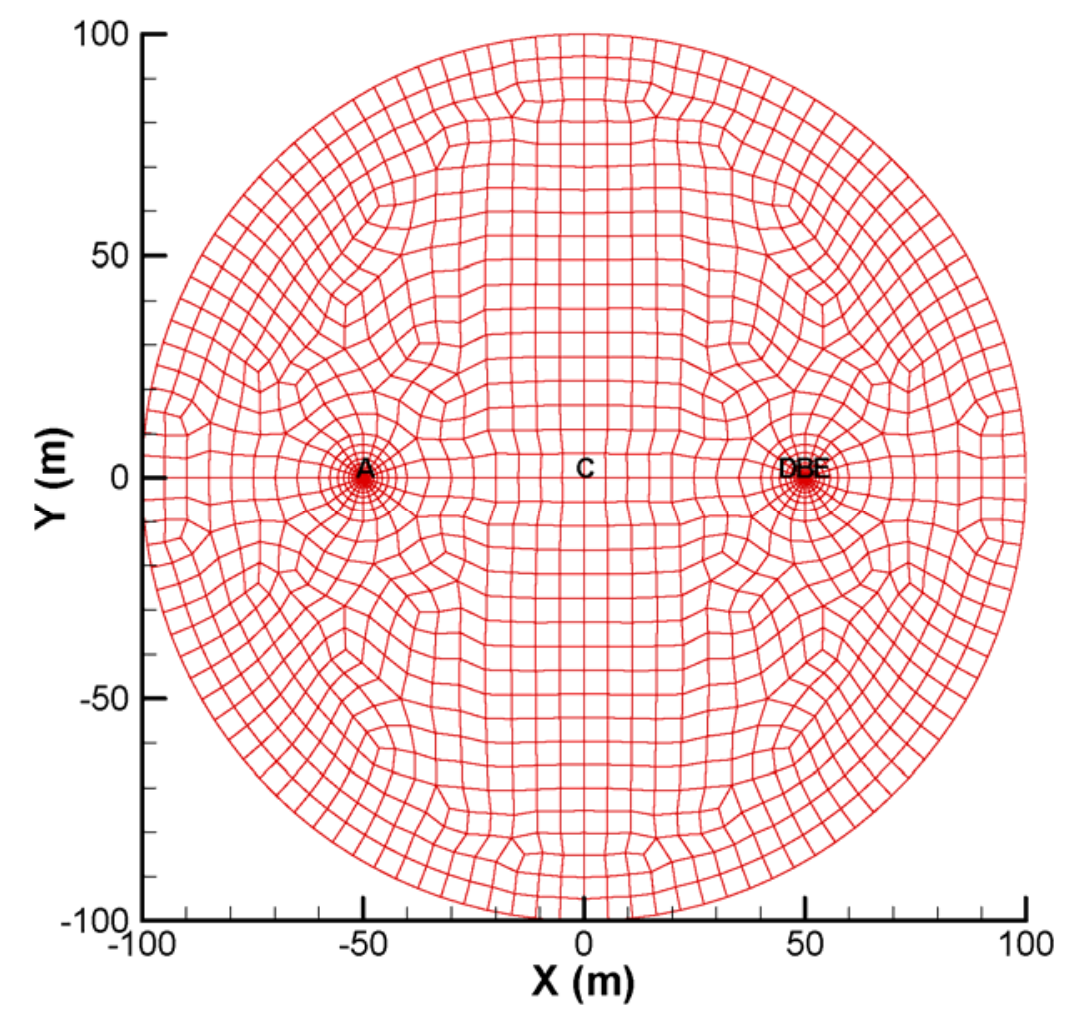

Figure 5.33. Discretization of a circular planar fracture: $\mathrm{A}$ is the injection well and $\mathrm{B}$ is the extraction well.

Table 4. Data set used in the numerical example.

\begin{tabular}{|l|l|}
\hline Parameter & Value \\
\hline Shear modulus $G(\mathrm{GPa})$ & 15.0 \\
\hline Poisson's ratio $v$ & 0.25 \\
\hline Fluid viscosity $\mu_{f}\left(\mathrm{~N} . \mathrm{s} / \mathrm{m}^{2}\right)$ & 0.001 \\
\hline Fluid diffusivity $c_{f}\left(\mathrm{~m}^{2} / \mathrm{s}\right)$ & $10^{-5}$ \\
\hline Biot's coefficient $\alpha$ & 0.47 \\
\hline Fluid density $\rho_{f}\left(\mathrm{~kg} / \mathrm{m}^{3}\right)$ & 1000 \\
\hline Rock density $\rho_{r}\left(\mathrm{~kg} / \mathrm{m}^{3}\right)$ & 2650 \\
\hline Rock permeability $\kappa\left(\mathrm{m}^{2}\right)$ & $10^{-18}$ \\
\hline Fluid heat capacity $c_{f}(\mathrm{~J} / \mathrm{kg} \mathrm{K})$ & 4200 \\
\hline Rock heat capacity $c_{r}(\mathrm{~J} / \mathrm{kg} \mathrm{K})$ & 800 \\
\hline Rock thermal conductivity $K_{r}(\mathrm{~W} / \mathrm{m} \mathrm{K})$ & 2.9 \\
\hline Rock linear thermal expansion coefficient $\alpha_{T}(1 / \mathrm{K})$ & $8 \times 10^{-6}$ \\
\hline Injection fluid temperature $T_{i n j}(\mathrm{~K})$ & 300 \\
\hline Rock temperature $T_{r}(\mathrm{~K})$ & 420 \\
\hline Fluid injection rate $Q_{i n j}\left(\mathrm{~m}^{3} / \mathrm{s}\right)$ & 0.01 \\
\hline Initial average fracture aperture for flow $w_{o}(\mathrm{~m})$ & $5 \times 10^{-5}$ \\
\hline Joint normal stiffness $K_{n}(\mathrm{~Pa} / \mathrm{m})$ & $10^{11}$ \\
\hline
\end{tabular}




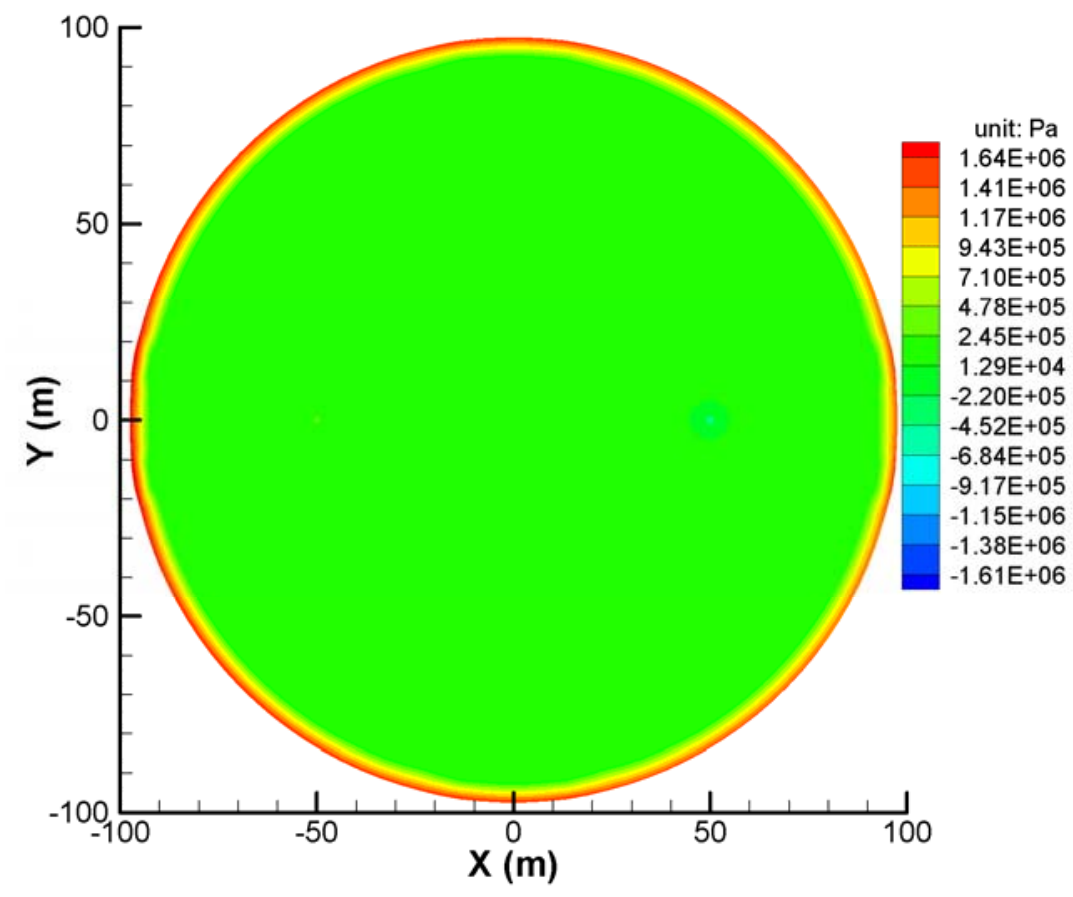

(a) $\mathrm{t}=1.0 \times 10^{5} \mathrm{~s}$

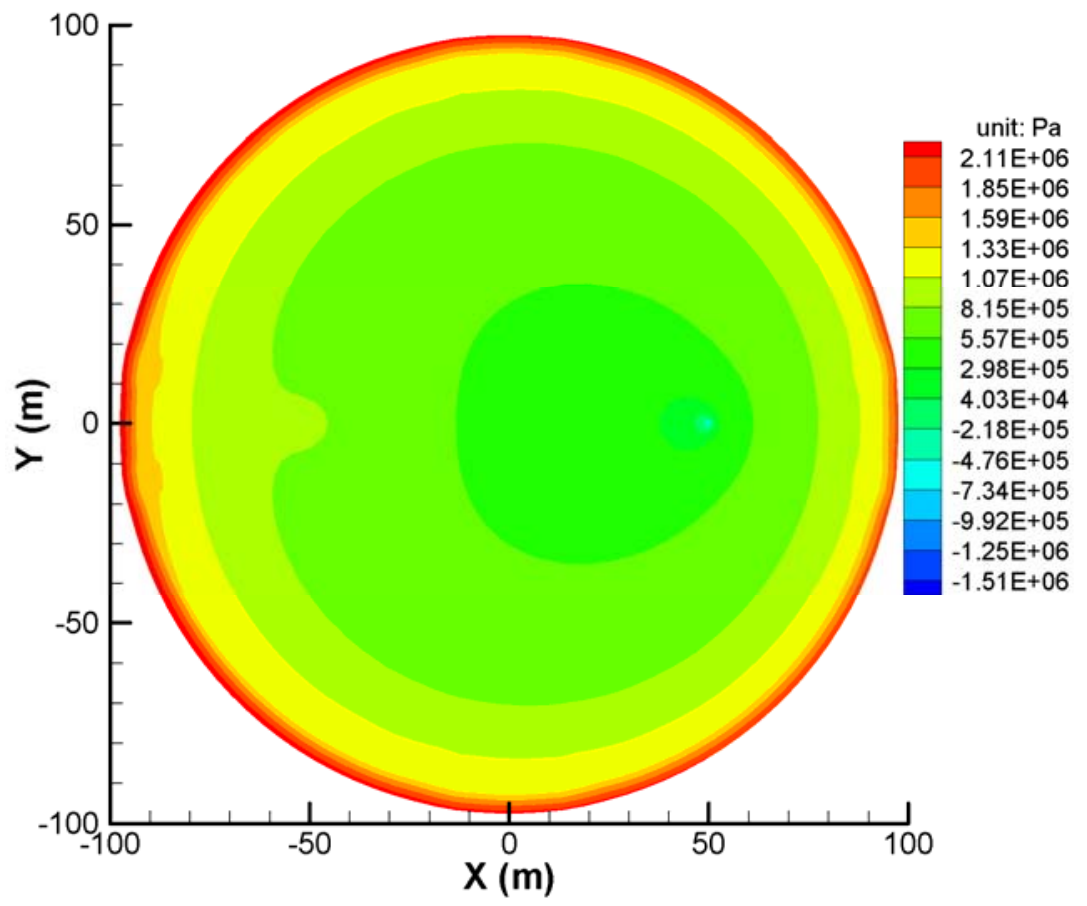

(b) $\mathrm{t}=1.0 \times 10^{7} \mathrm{~s}$ 


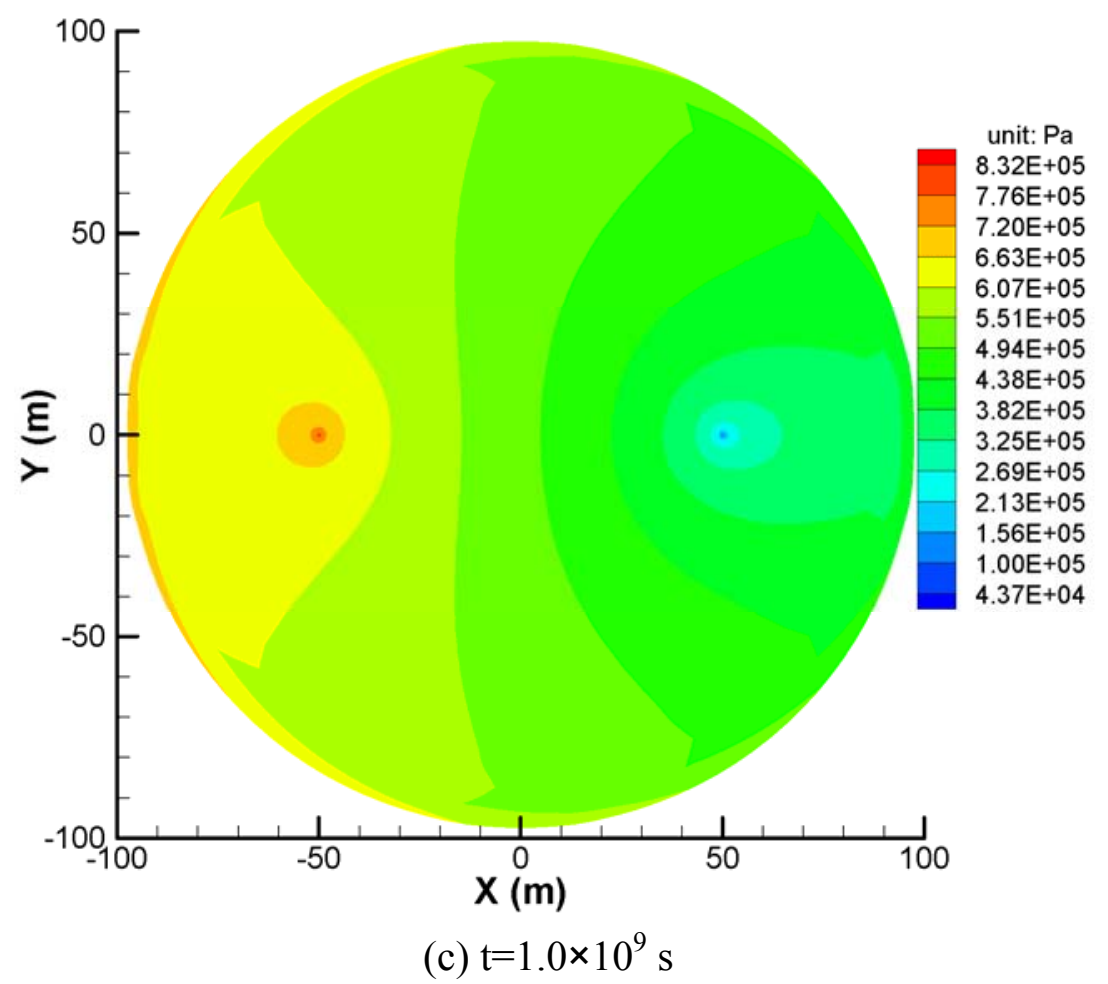

Figure 5.34. Distributions of poroelastic stress $\sigma_{\mathrm{zz}}$ from poro-thermoelastic model after different injection times: (a) $\mathrm{t}=1.0 \times 10^{5} \mathrm{~s}$; (b) $\mathrm{t}=1.0 \times 10^{7} \mathrm{~s}$; (c) $\mathrm{t}=1.0 \times 10^{9} \mathrm{~s}$.

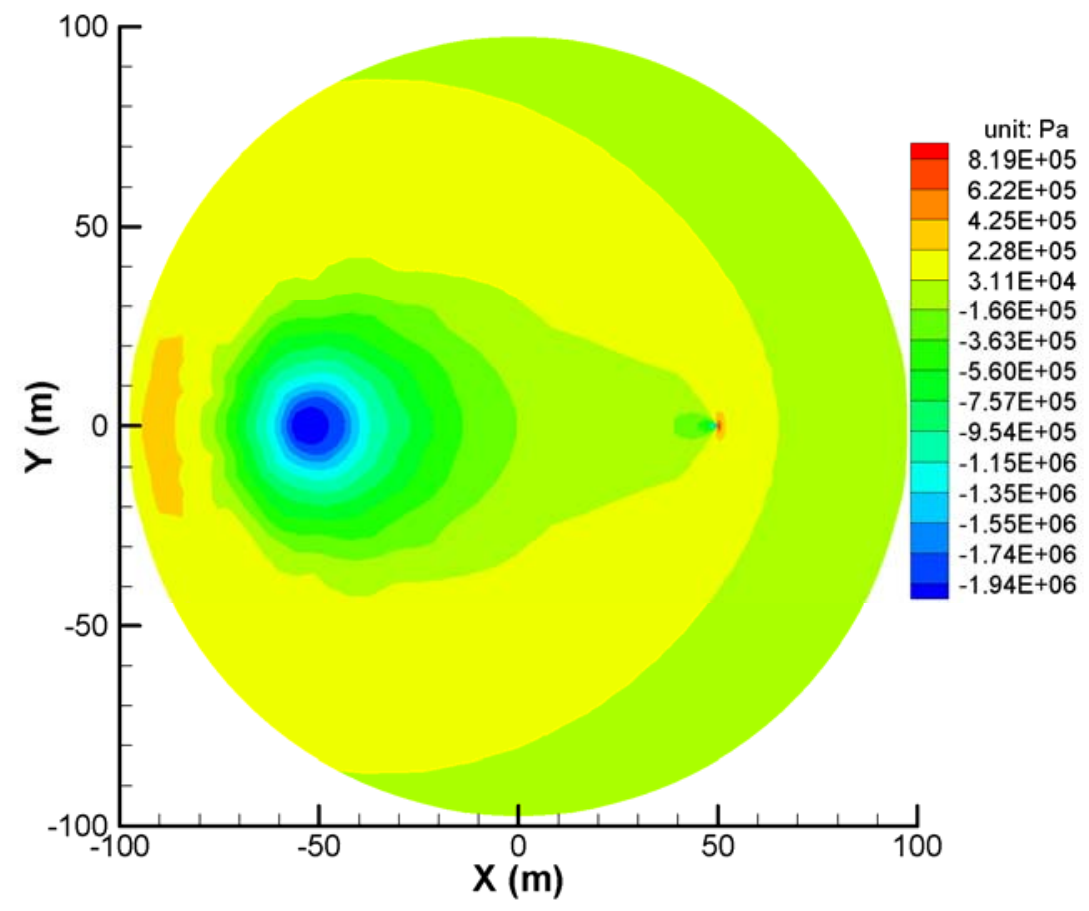

(a) $\mathrm{t}=1.0 \times 10^{5} \mathrm{~s}$ 


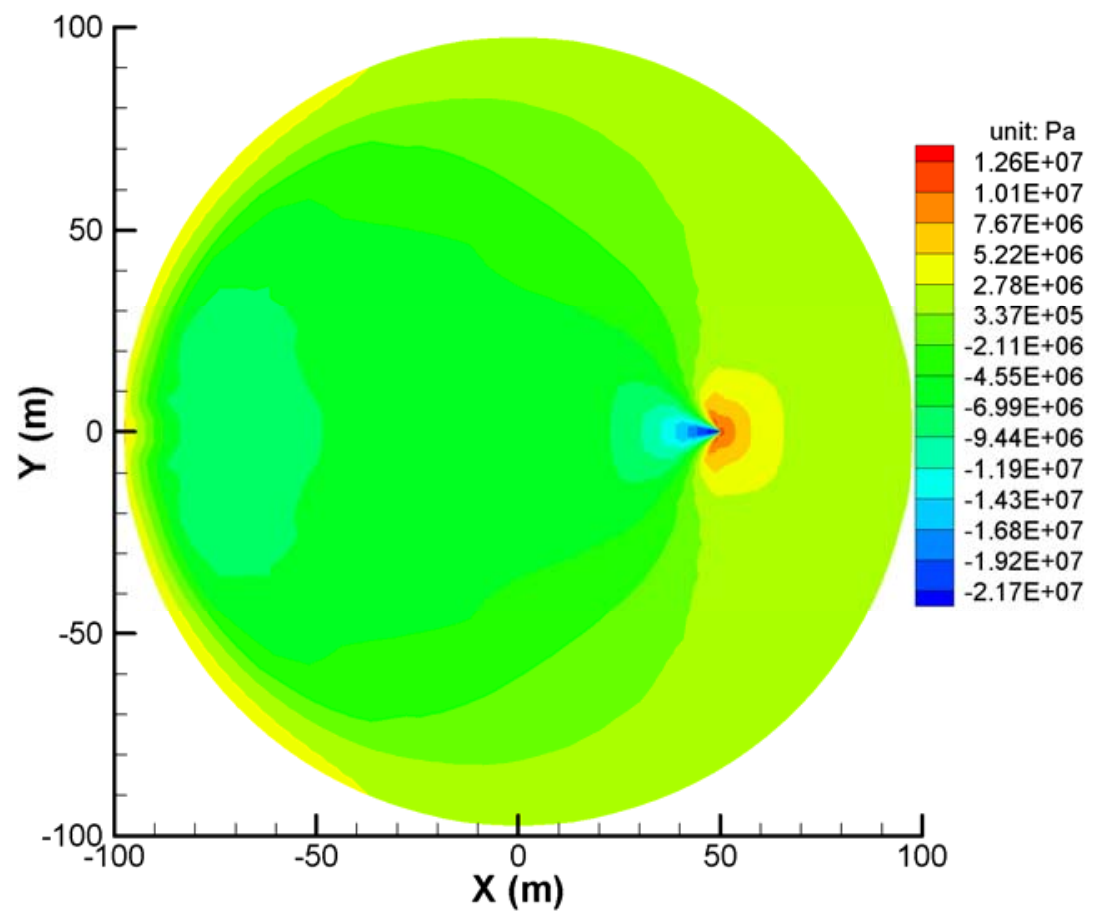

(b) $\mathrm{t}=1.0 \times 10^{7} \mathrm{~s}$

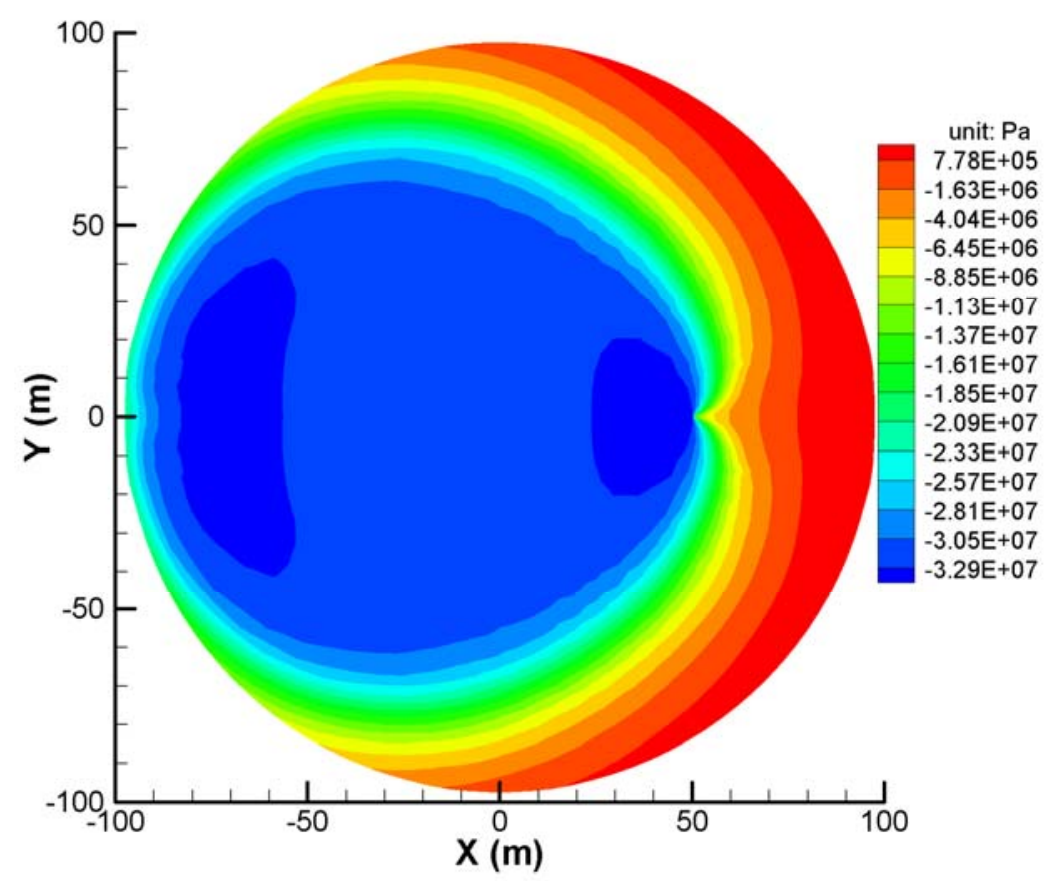

(c) $\mathrm{t}=1.0 \times 10^{9} \mathrm{~s}$

Figure 5.35. Distributions of thermoelastic stress $\sigma_{z z}$ from poro-thermoelastic model after different injection times: (a) $\mathrm{t}=1.0 \times 10^{5} \mathrm{~s}$; (b) $\mathrm{t}=1.0 \times 10^{7} \mathrm{~s}$; (c) $\mathrm{t}=1.0 \times 10^{9} \mathrm{~s}$. 


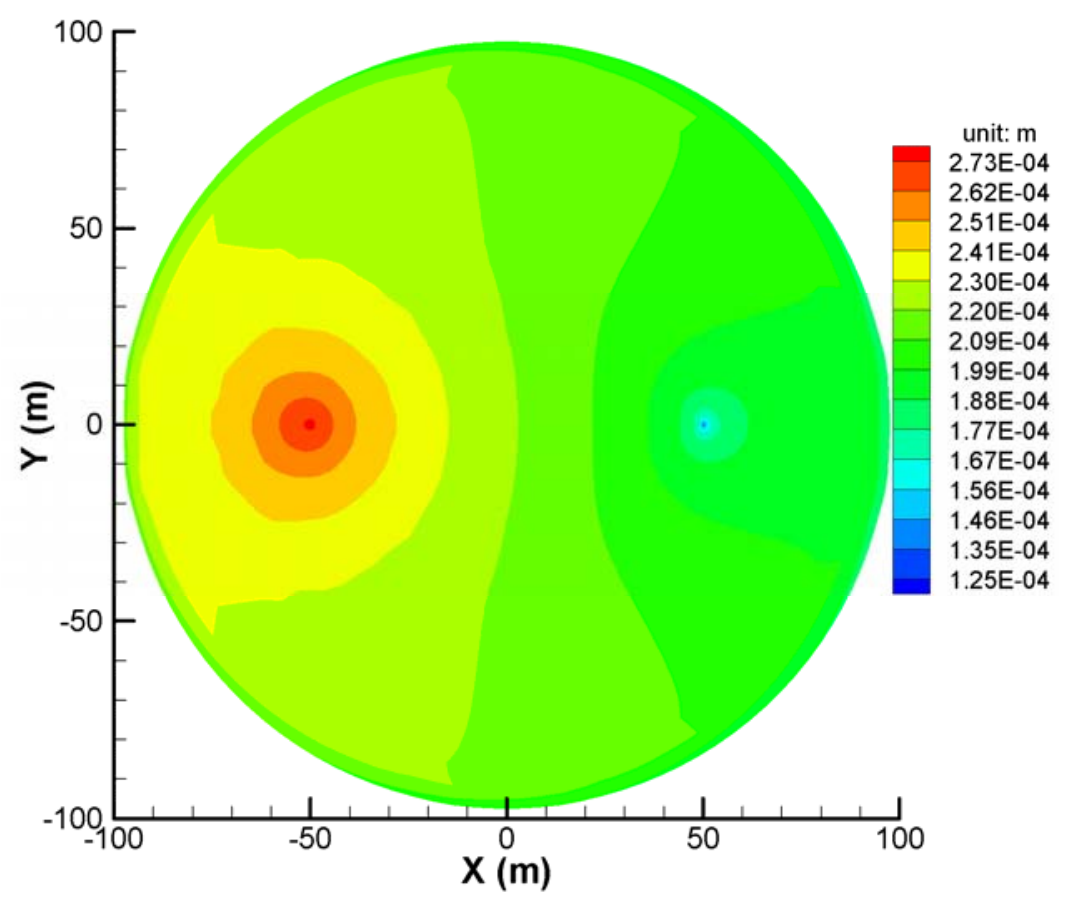

(a) $\mathrm{t}=1.0 \times 10^{5} \mathrm{~s}$

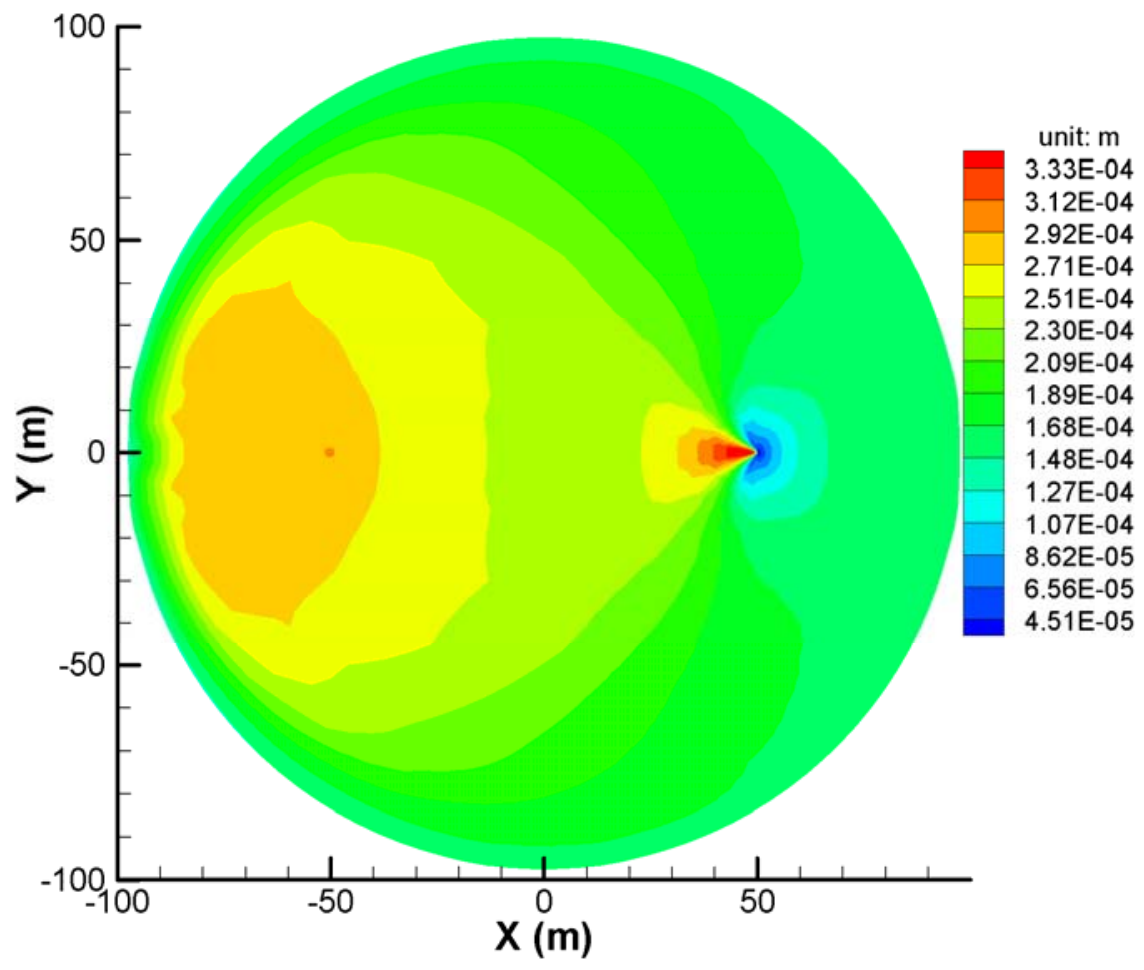

(b) $\mathrm{t}=1.0 \times 10^{7} \mathrm{~s}$ 


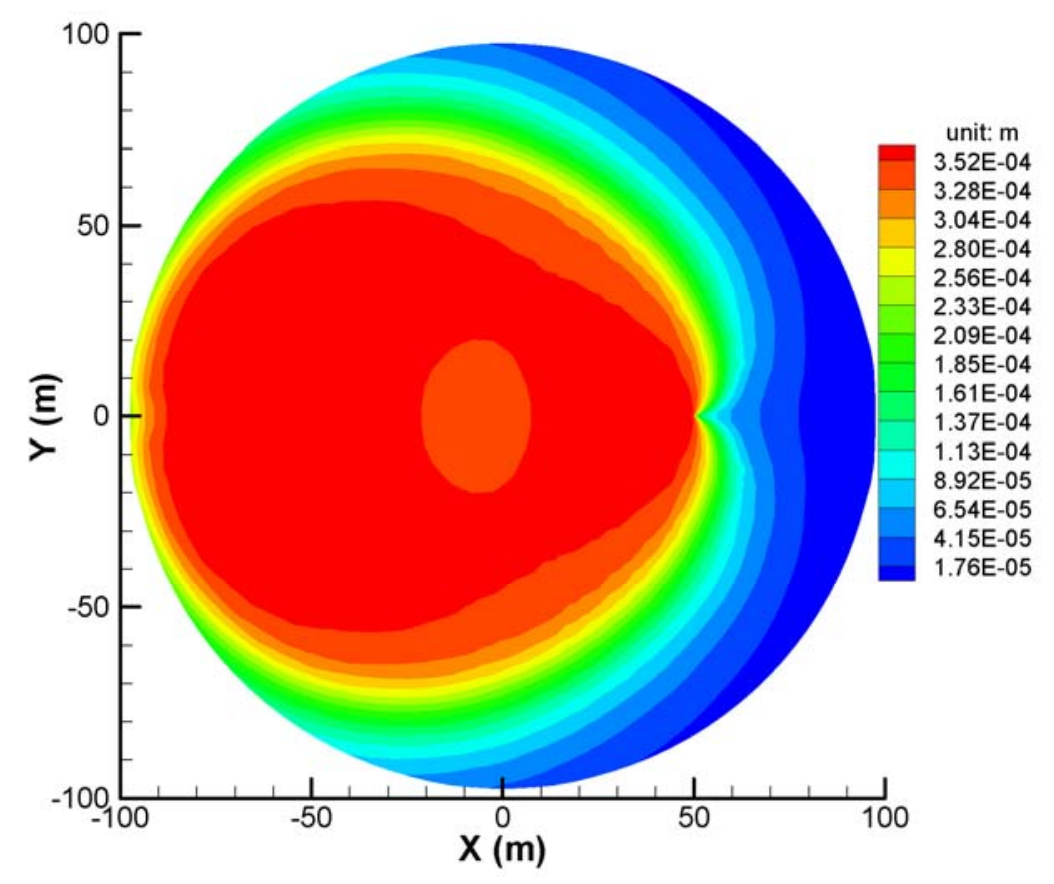

(c) $\mathrm{t}=1.0 \times 10^{9} \mathrm{~s}$

Figure 5.36. Distributions of the fracture aperture increment from poro-thermoelastic model after different injection times: (a) $\mathrm{t}=1.0 \times 10^{5} \mathrm{~s}$; (b) $\mathrm{t}=1.0 \times 10^{7} \mathrm{~s}$; (c) $\mathrm{t}=1.0 \times 10^{9} \mathrm{~s}$.

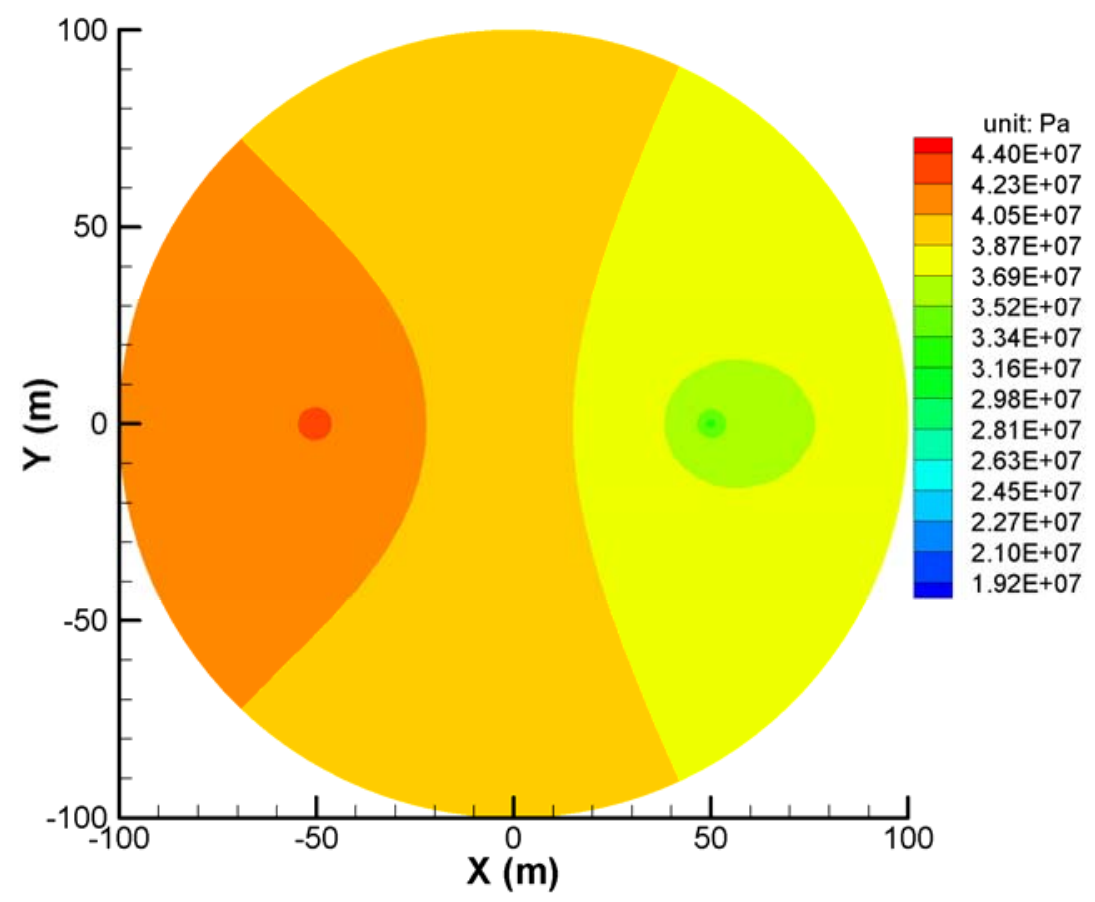

(a) $\mathrm{t}=1.0 \times 10^{5} \mathrm{~s}$ 


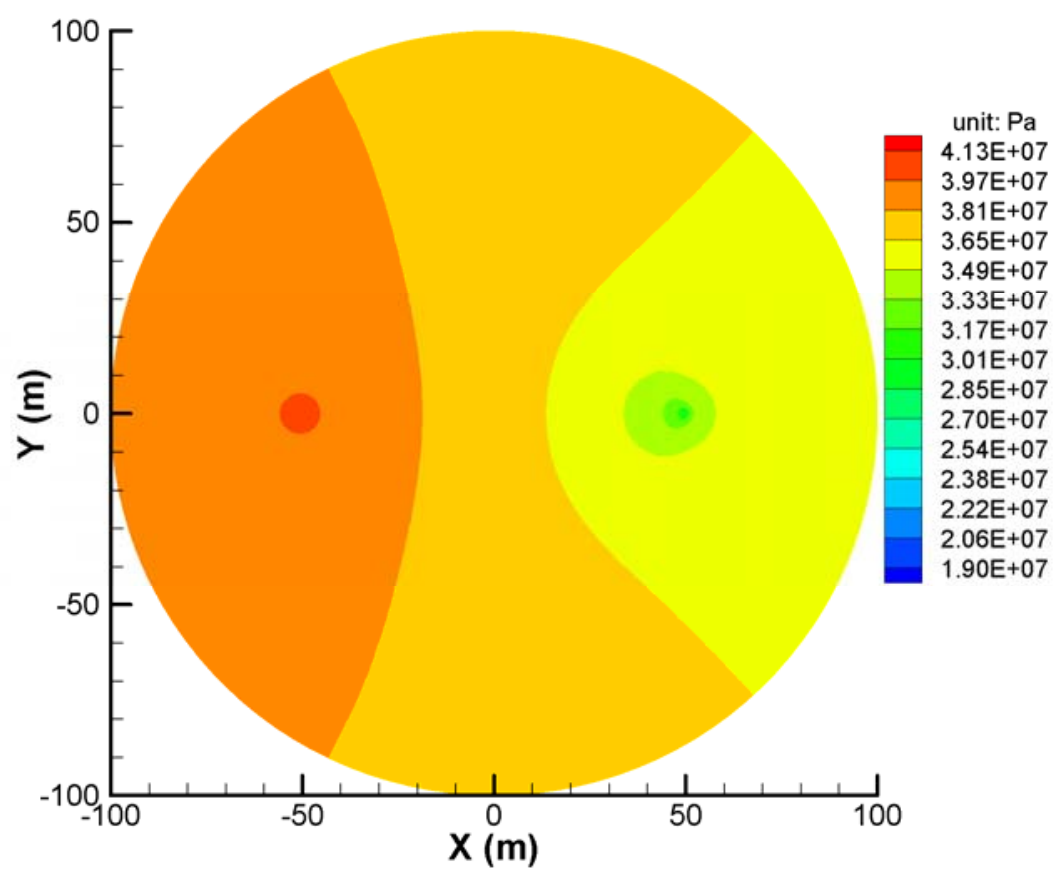

(b) $\mathrm{t}=1.0 \times 10^{7} \mathrm{~s}$

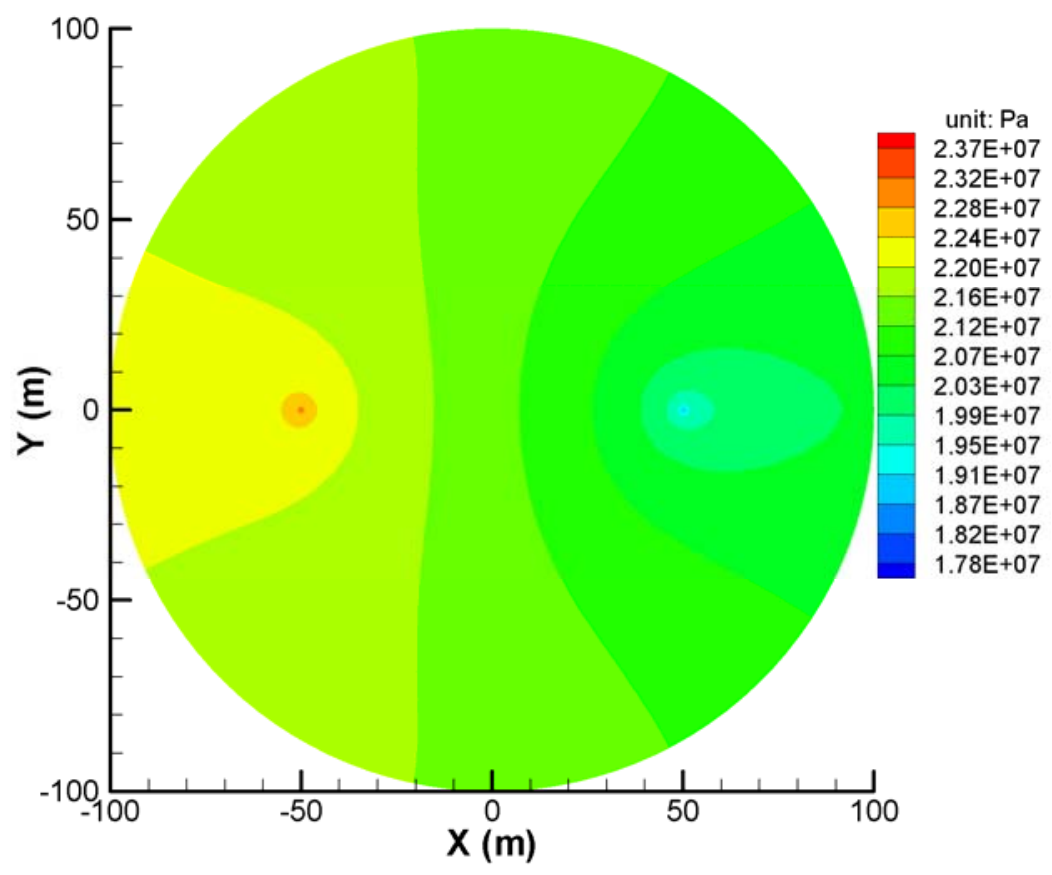

(c) $\mathrm{t}=1.0 \times 10^{9} \mathrm{~s}$

Figure 5.37. Distributions of fluid pressure in the fracture from poro- and thermoelastic model after different injection times: (a) $\mathrm{t}=1.0 \times 10^{5} \mathrm{~s}$; (b) $\mathrm{t}=1.0 \times 10^{7} \mathrm{~s}$; (c) $\mathrm{t}=1.0 \times 10^{9} \mathrm{~s}$. 


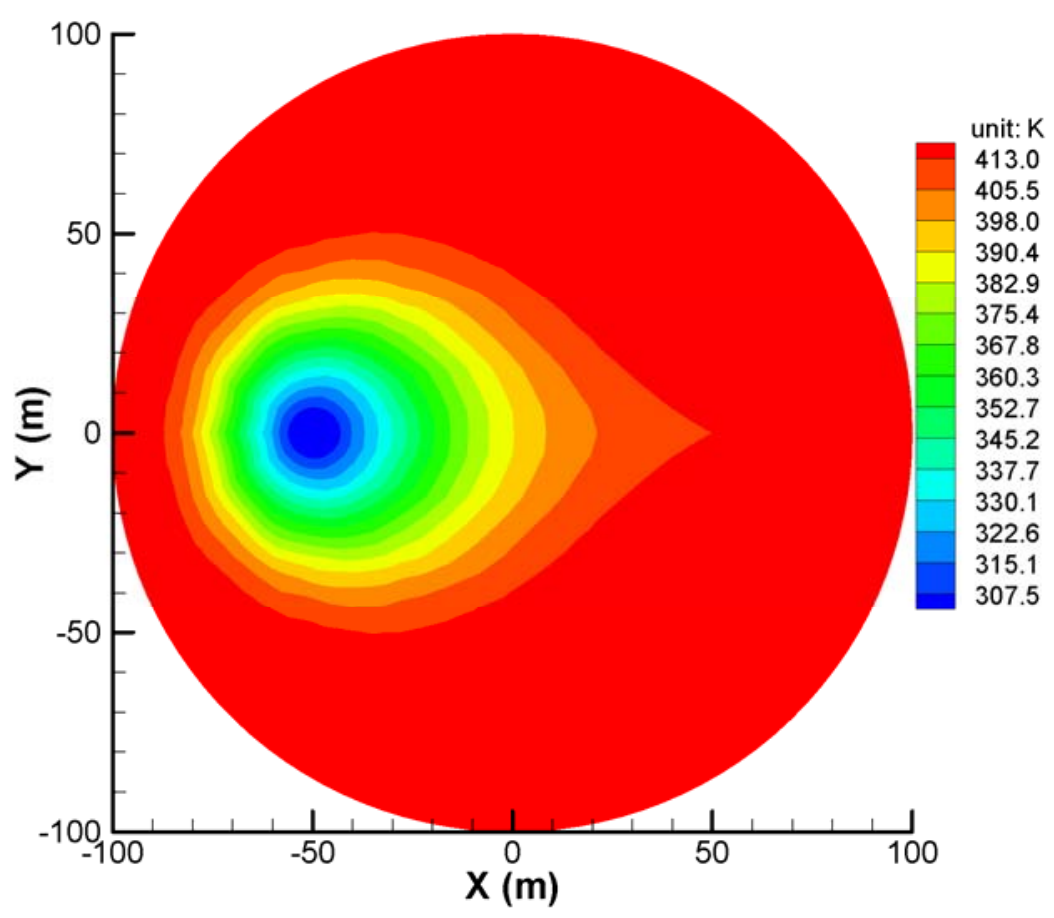

(a) $\mathrm{t}=1.0 \times 10^{5} \mathrm{~s}$.

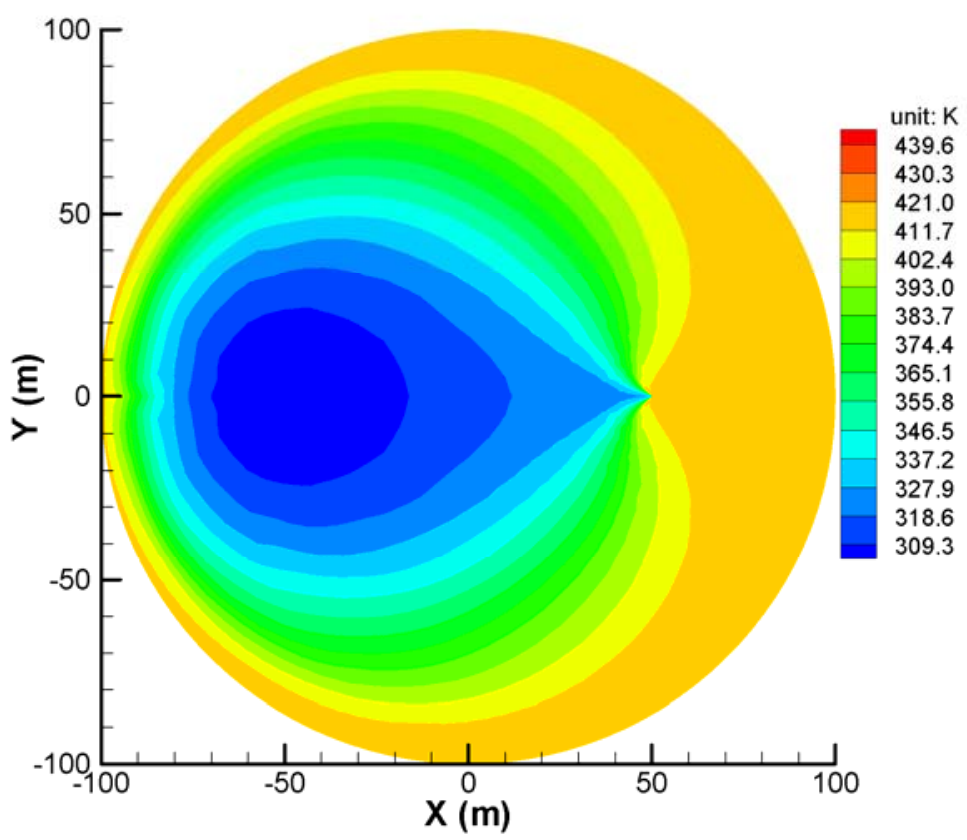

(b) $\mathrm{t}=1.0 \times 10^{7} \mathrm{~s}$ 


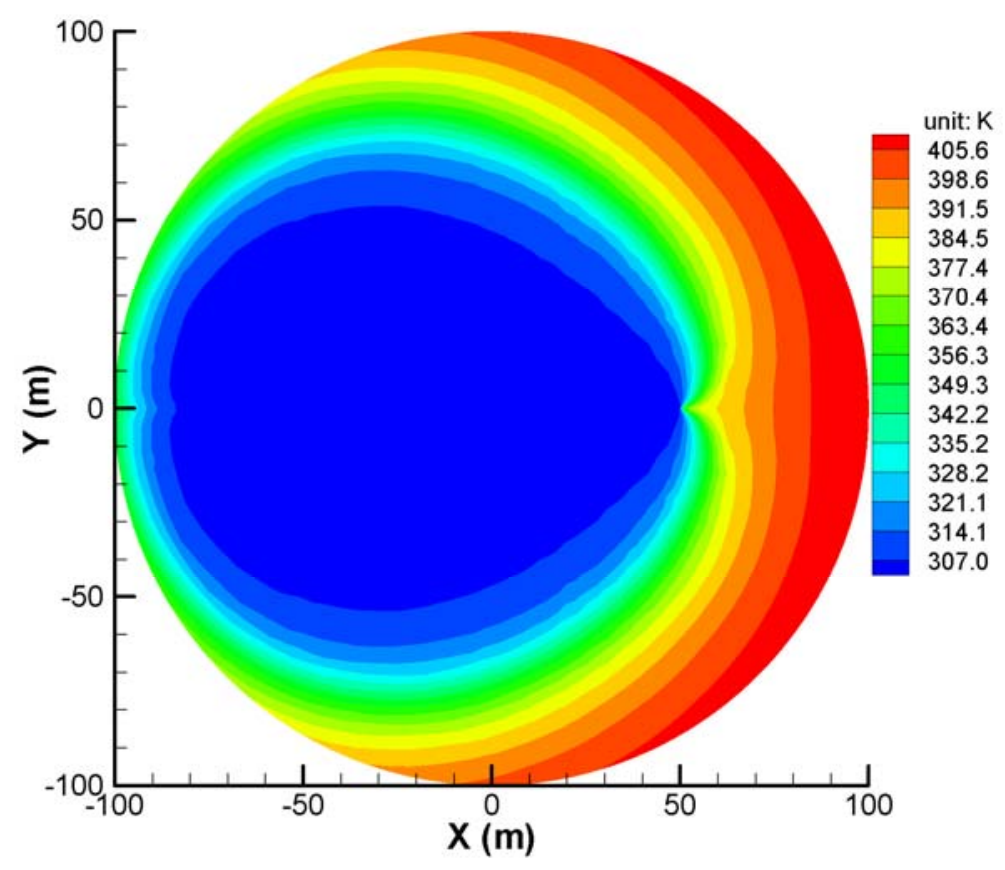

(c) $\mathrm{t}=1.0 \times 10^{9} \mathrm{~s}$

Figure 5.38. Distributions of fluid temperature in the fracture from poro-thermoelastic model after different injection times: (a) $\mathrm{t}=1.0 \times 10^{5} \mathrm{~s}$; (b) $\mathrm{t}=1.0 \times 10^{7} \mathrm{~s}$; (c) $\mathrm{t}=1.0 \times 10^{9} \mathrm{~s}$.

Figure 5.37 shows the distributions of the fluid pressures in the fracture corresponding to different fluid injection times. The fracture pressures decrease with the time because of the increase of the fracture apertures which is mainly due to the thermal effect when the fluid injection time is relative long. Figure 5.38 shows the temperature distributions at different fluid injection times. It is observed that the low temperature zone is around the injection well at the early times, and then spreads to the extraction well with the continuation of the fluid injection into the fracture.

To understand the physical processes involved in the fluid injection and extraction process better, in the following, we examine the developments of fracture pressures and fracture aperture widths on points $\mathrm{A}(-50,0), \mathrm{C}(0,0), \mathrm{D}(49.8,0)$ and $\mathrm{E}(50.2,0)$, where point $\mathrm{A}$ is the location of injection well, point $\mathrm{C}$ is the center of the circular fracture, and points $\mathrm{D}$ and $\mathrm{E}$ are respectively at the locations just before and behind the extraction well.

Figure 5.39 and 5.40 present the variations of the fracture aperture and fluid pressure on point A with time by considering the poroelastic, thermoelastic, and their combined effect, respectively. The poroelastic model again shows that the fracture aperture increases significantly at the early time stage, and then slightly decreases after some times; while the fracture pressure increases first, then unlike the fracture aperture, continues to increase with the time. As we used a very small initial fracture aperture $\left(5 \times 10^{-5} \mathrm{~m}\right)$, the initial fracture pressure is relatively large compared to the initial reservoir pressure (note that the fracture is not open since the fluid pressure is still less than the min- in-situ stress and there is no shear slip as it is not considered in this example). As a result, there is a 
large fluid leakoff from the fracture into the reservoir, making the fluid pressure in the poroelastic case much smaller than that in the thermoelastic case in which no fluid leakoff occurs. However, the fluid leakoff decreases with the elapse of the fluid injection time, resulting in less and less influence of the fluid leakoff on the fracture pressure with the time. During the early time stage, the fracture closure due to the leakoff induced poroelastic stresses is not as pronounced as the fracture opening due to the increase of fracture pressures. This may be used to explain why both the fracture aperture and fracture pressure increase during this period. After some injection times, the fluid leakoff will be very small as the difference between the fracture pressure and reservoir pressure becomes small. During this period, the fracture closure due to the poroelastic stresses is dominant. The decrease of the fracture aperture also leads to larger fracture pressure.

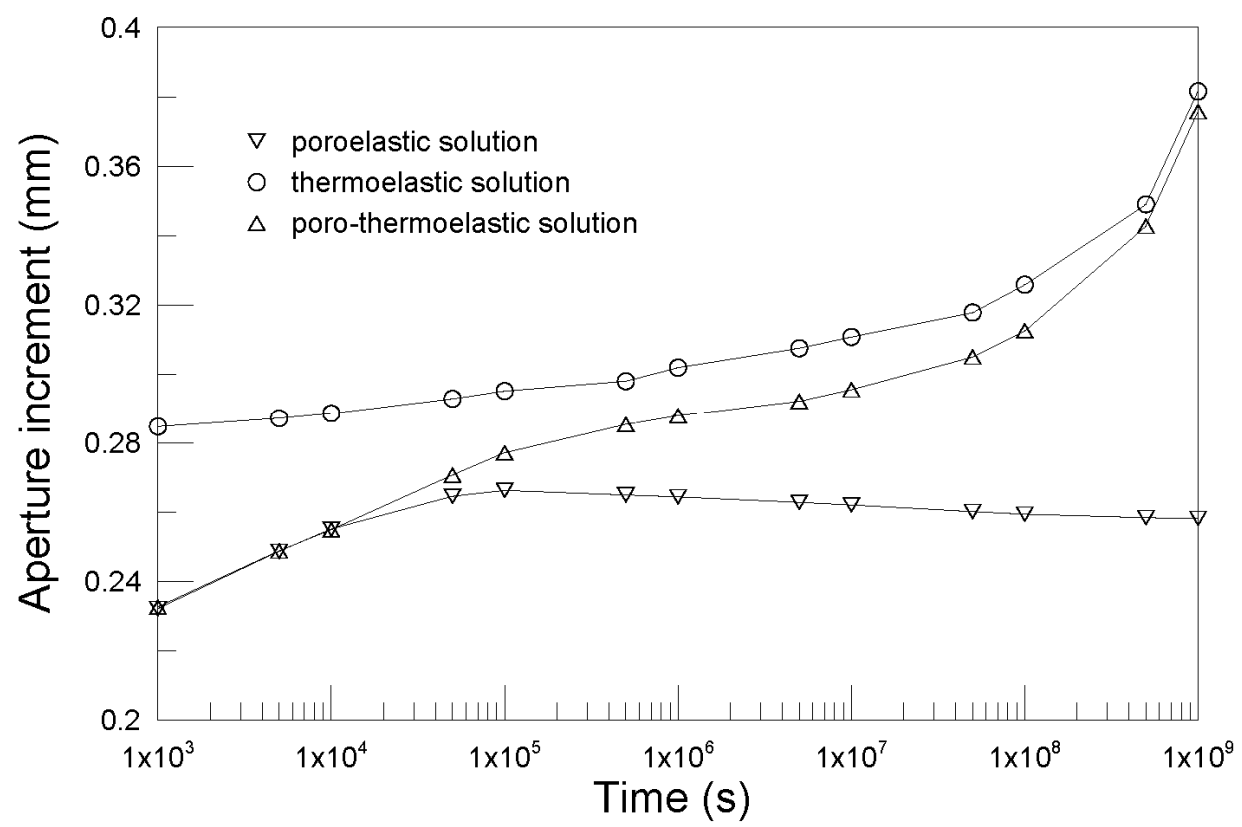

Figure 5.39. Comparison of fracture aperture increments at point A from poroelastic, thermoelastic, and poro-thermoelastic models in the fluid injection and extraction process. 


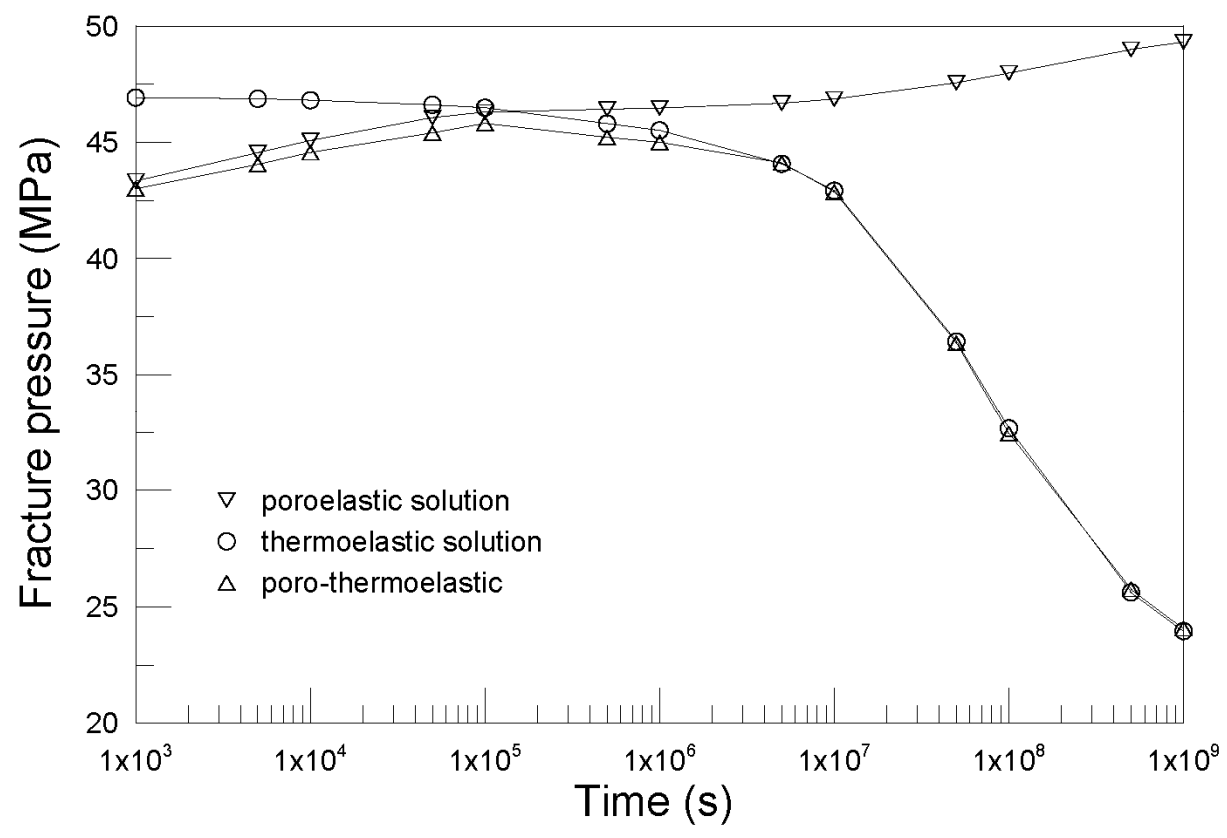

Figure 5.40. Comparison of fluid pressures at the location of point A from the poroelastic, thermoelastic, and poro-thermoelastic models during the fluid injection and extraction process.

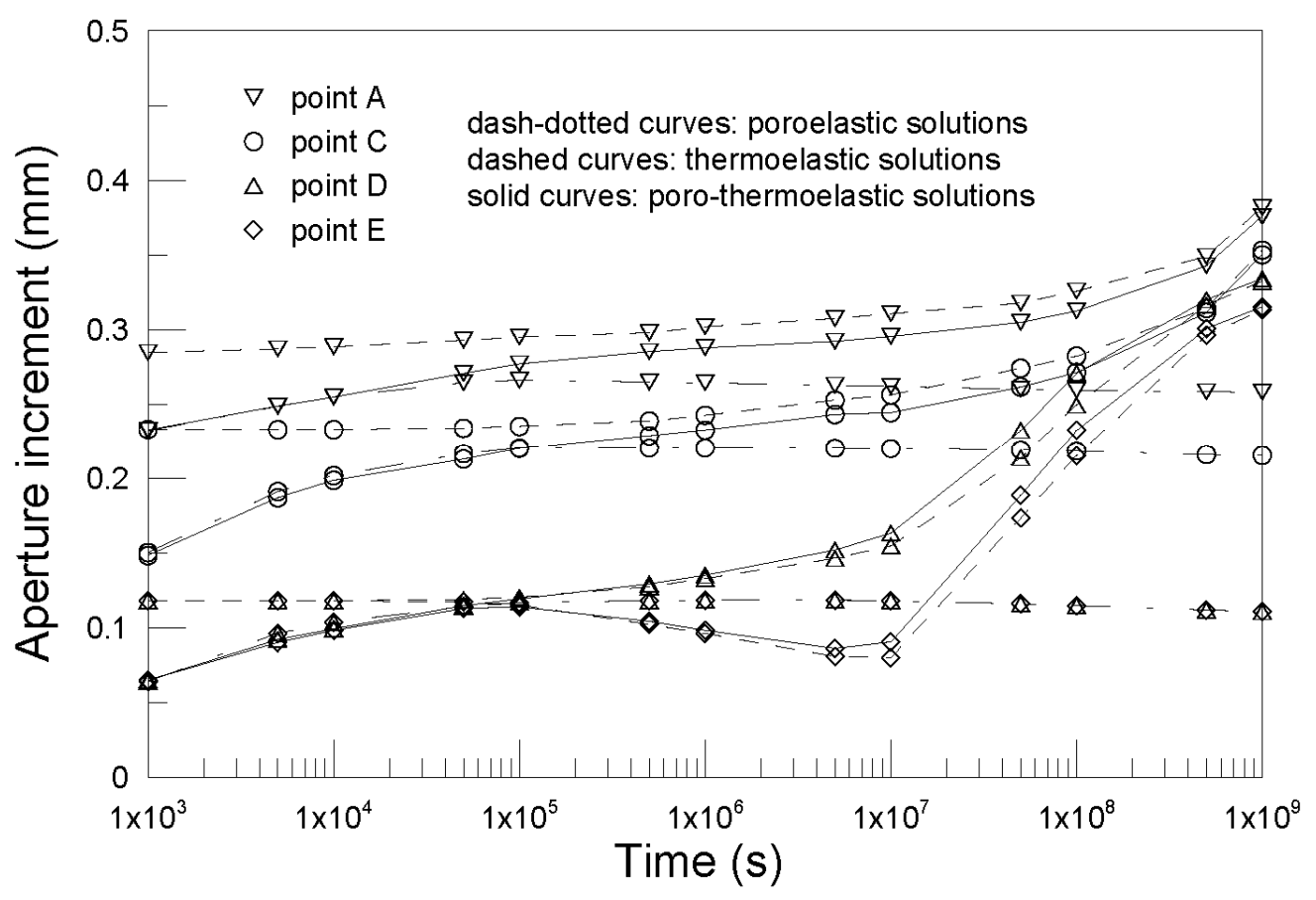

Figure 5.41. Comparison of fracture aperture at points A, C, D and E during injection and extraction process from poroelastic, thermoelastic and combined models.

The thermoelastic solution predicts that the fracture aperture on point A increases gradually as time increases due to the increased tensile thermo-stresses at the fracture 
surface as well as in the reservoir matrix. It is observed that early on, the fracture pressure on point $\mathrm{A}$ is increasing very slightly, but the aperture on point $\mathrm{A}$ increases at a higher rate. The reason is that the fracture is not heated uniformly at the early time stage, resulting the distributions of the fracture apertures are also not uniform (see Figs. 36 and 37). At the early time stage, the area around the injection well is heated first, which makes the fracture apertures at this area are larger than most of the other areas. That's why the incremental rate of the fracture aperture is higher than that of the fracture pressure on point $\mathrm{A}$. After a long time of fluid injection, the fracture pressure on point $\mathrm{A}$ drops fast as the increment of fracture aperture mainly occurs during that period.

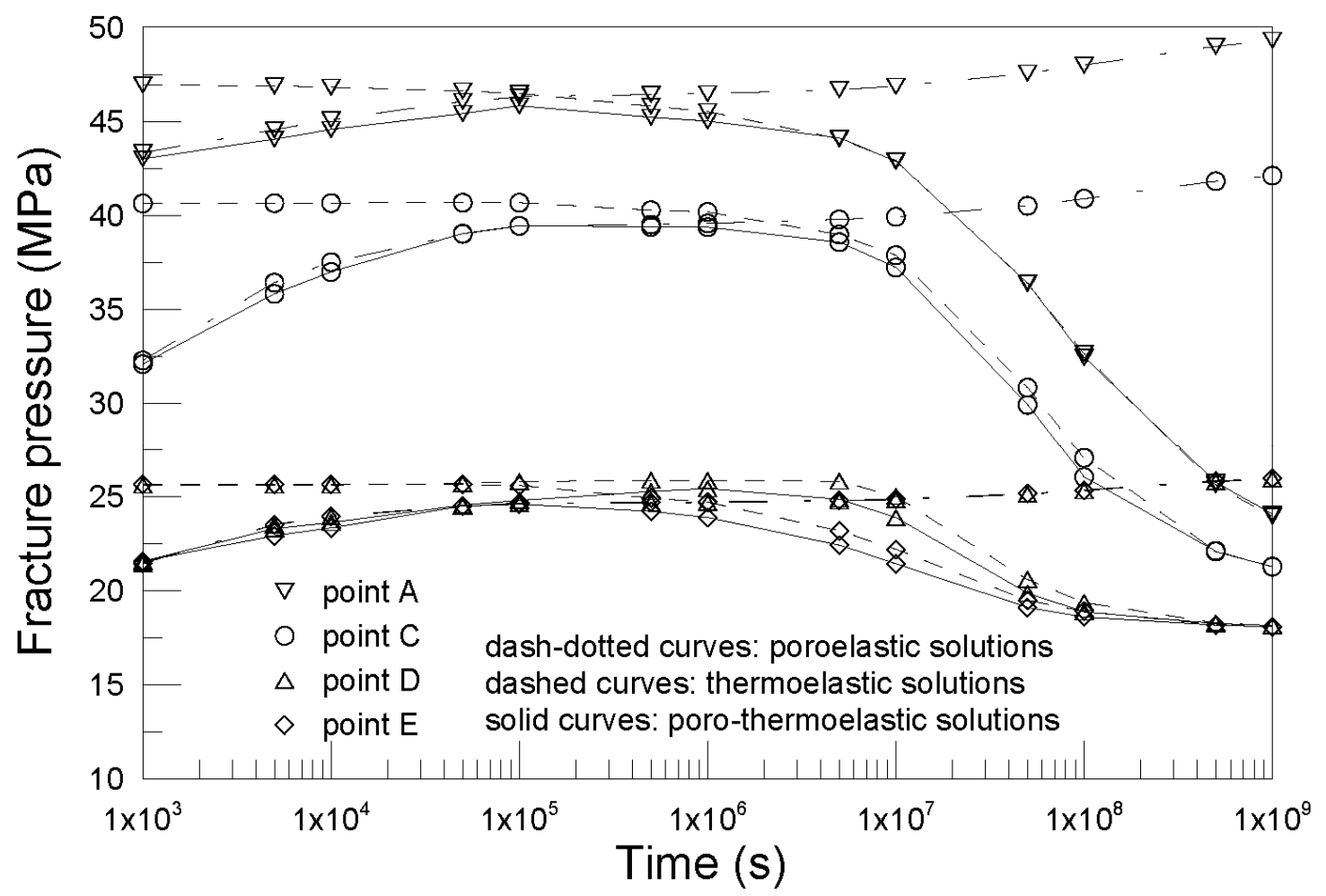

Figure 5.42. Comparison of fluid pressures at points A, C, D and E from the poroelastic, thermoelastic and combined models for injection and extraction process.

The combined poro- and thermoelastic solutions show that at the early time stage, both the fracture aperture and pressure from the poro-thermoelastic model are very close to the poroelastic solutions, which indicates the influence of the fluid leakoff is dominant at this time; while both poro-thermoelastic solutions tend to the thermoelastic solutions after a long time of fluid injection when the thermo-effect is dominant. However, even after a very long time of fluid injection, the fracture apertures predicted by the porothermoelastic model is still smaller than that predicted by the thermoelastic model. The difference between them is caused by the fluid leakoff induced fracture closure. Through the poro- 
thermoelastic results, we found that the poromechanical and thermomechanical processes occur at different time scales for the present fluid injection and extraction problem. The thermomechanical coupling is important in the time scale of months to years; however, the poromechanical coupling occurs much rapidly after the fluid injection begins. These conclusions are in agreement with those in previous literature [1].

Figsures 41 and 42 present the variations of fracture apertures and pressures on points A, $\mathrm{C}, \mathrm{D}$ and $\mathrm{E}$ with time from the poroelastic, thermoelastic and poro-thermoelastic models. The variations of the results on points $\mathrm{C}, \mathrm{D}$ and $\mathrm{E}$ are similar to those on point $\mathrm{A}$. However, it should be noted that there is a little difference for the thermoelastic and porothermoelastic results on E, which is located just behind the extraction well. When we include the heat exchange between the injected fluid and reservoir matrix, relatively large tensile stresses are found just before the extraction well and compressive stresses just behind the extraction wells. As a result, we found relatively larger fracture aperture on point $\mathrm{D}$ and smaller fracture aperture on point $\mathrm{E}$ after some times. However, the fracture apertures on points $\mathrm{D}$ and $\mathrm{E}$ become closer when the fluid injection time is long enough that no large compressive thermo-stresses appear just behind the extraction as shown in Figure 35(c).

\subsubsection{Irregularly-shaped fracture}

The present numerical procedures is now applied to a fluid injection/extraction operation involving an arbitrarily shaped fracture. Figure 5.44 shows one such fracture and its discretization into surface elements. The fracture surface is divided into 1745 four-noded quadrilateral element and 1808 element nodes. It is assumed that the fracture plane is horizontal and the initial stresses and pore pressure field are the same as those in the preceding numerical example. The parameters in Table 4 are used here. The heat extraction operation involves three wells: injection well A with flow rate $Q_{i n j}=0.01 \mathrm{~m}^{3} / \mathrm{s}$, and two extraction wells $\mathrm{B}$ and $\mathrm{C}$ with the same initial reservoir pressure. The fully coupled poro-thermoelastic model is used to analyze the problem.

Figure 5.44 illustrates the distributions of fluid temperature in the fracture when the fluid injection times are $10^{5} \mathrm{~s}$ (1.16 days), $10^{7} \mathrm{~s}$ (115.7 days) and $10^{9} \mathrm{~s}$ (31.7 years). As expected, with the elapse of the time, the low temperature area spreads from the injection well to the extraction well and the temperature of the extracted fluid decreases. The cooling area spreads faster towards the extraction well that is closer to the injection well.

Figure 5.45 shows the distributions of the fracture aperture increment at different fluid injection times. The fracture aperture widths increase with the time. The fracture apertures are relatively larger around the injection well and near the extraction wells, and relatively small behind the extraction wells because of the influence of the thermostresses. Figure 5.46 shows the distributions of fracture fluid pressure corresponding to different fluid injection times. It is found that the fluid pressures in the fracture decreases significantly with the time as the fracture apertures increases in the fluid injection process. 


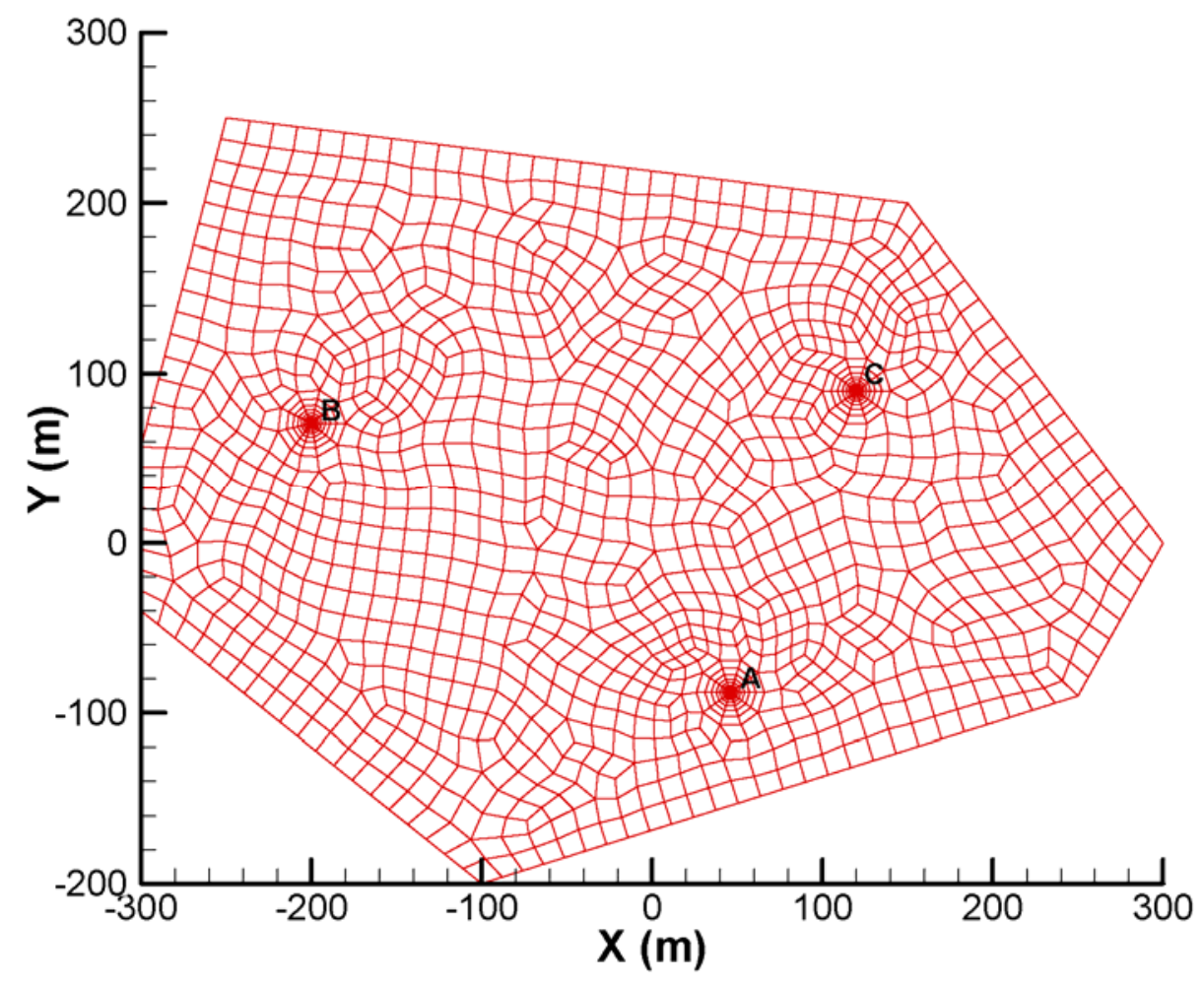

Figure 5.43. Discretization of an arbitrary shaped planar fracture, where A is fluid injection well, and $\mathrm{B}$ and $\mathrm{C}$ are fluid extraction wells.

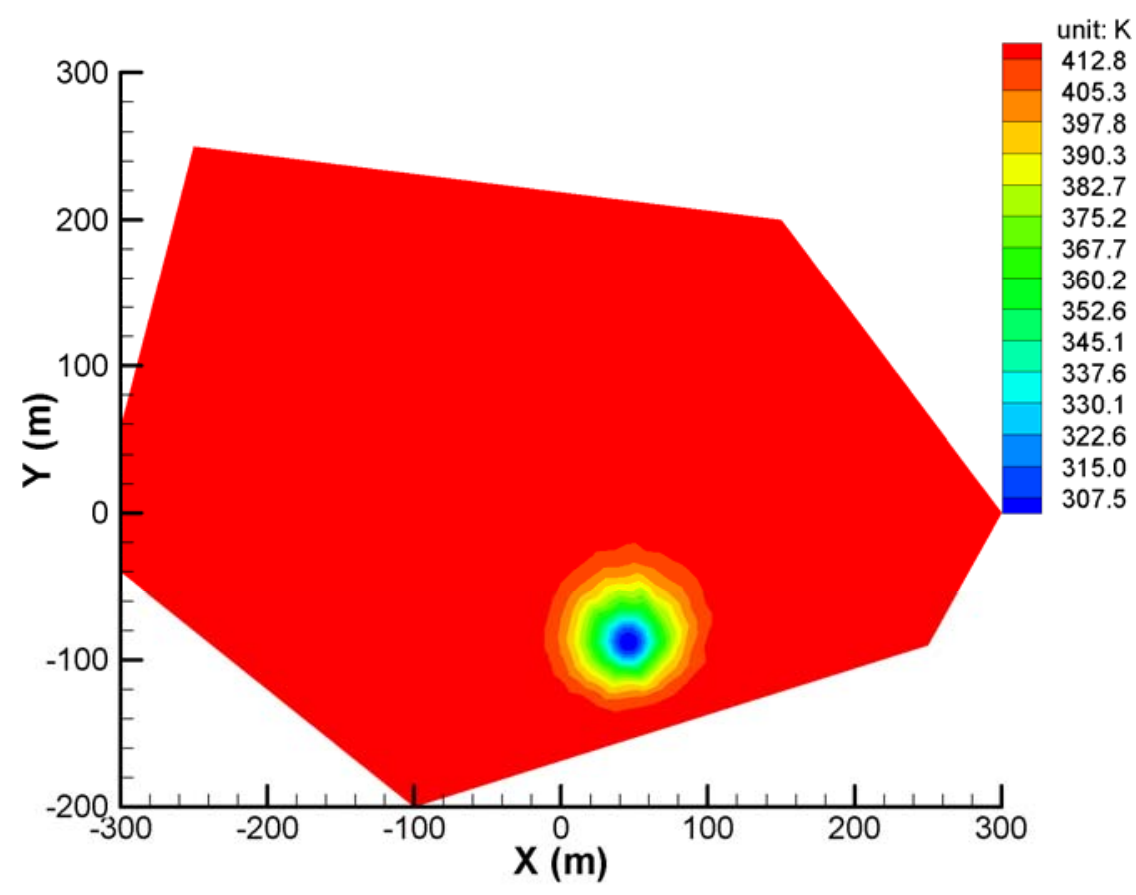

(a) $\mathrm{t}=1.0 \times 10^{5} \mathrm{~s}$ 


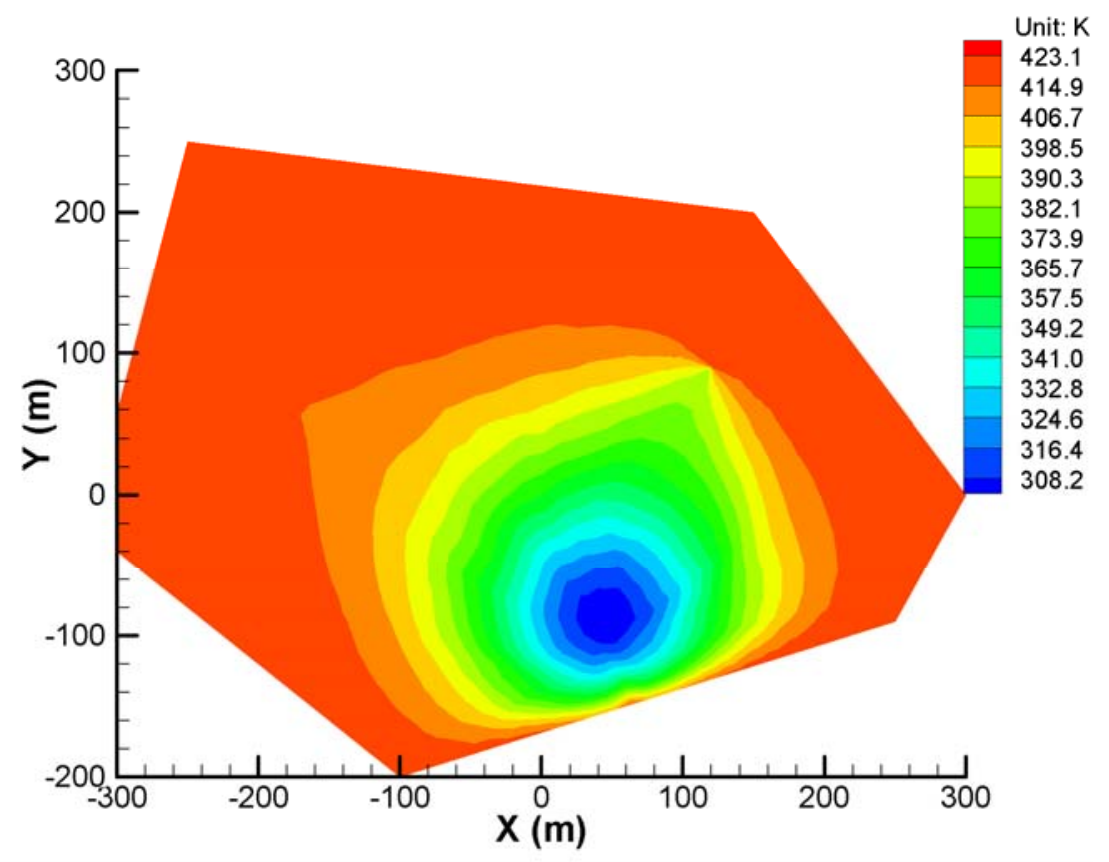

(b) $\mathrm{t}=1.0 \times 10^{7} \mathrm{~s}$

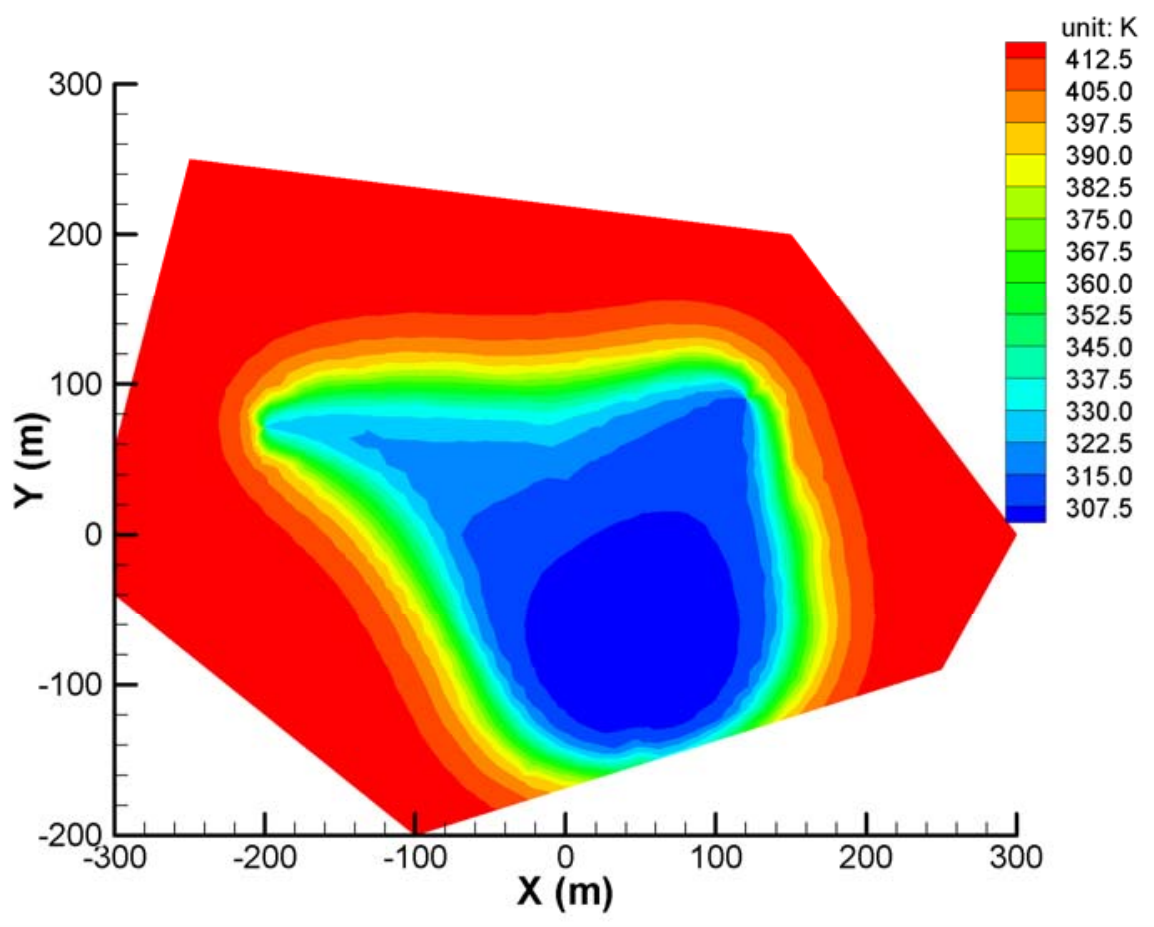

(c) $\mathrm{t}=1.0 \times 10^{9} \mathrm{~s}$

Figure 5.44. Distributions of fluid temperature in the fracture from poro-thermoelastic model after different fluid injection times: (a) $\mathrm{t}=1.0 \times 10^{5} \mathrm{~s}$; (b) $\mathrm{t}=1.0 \times 10^{7} \mathrm{~s}$; (c) $\mathrm{t}=1.0 \times 10^{9} \mathrm{~s}$. 


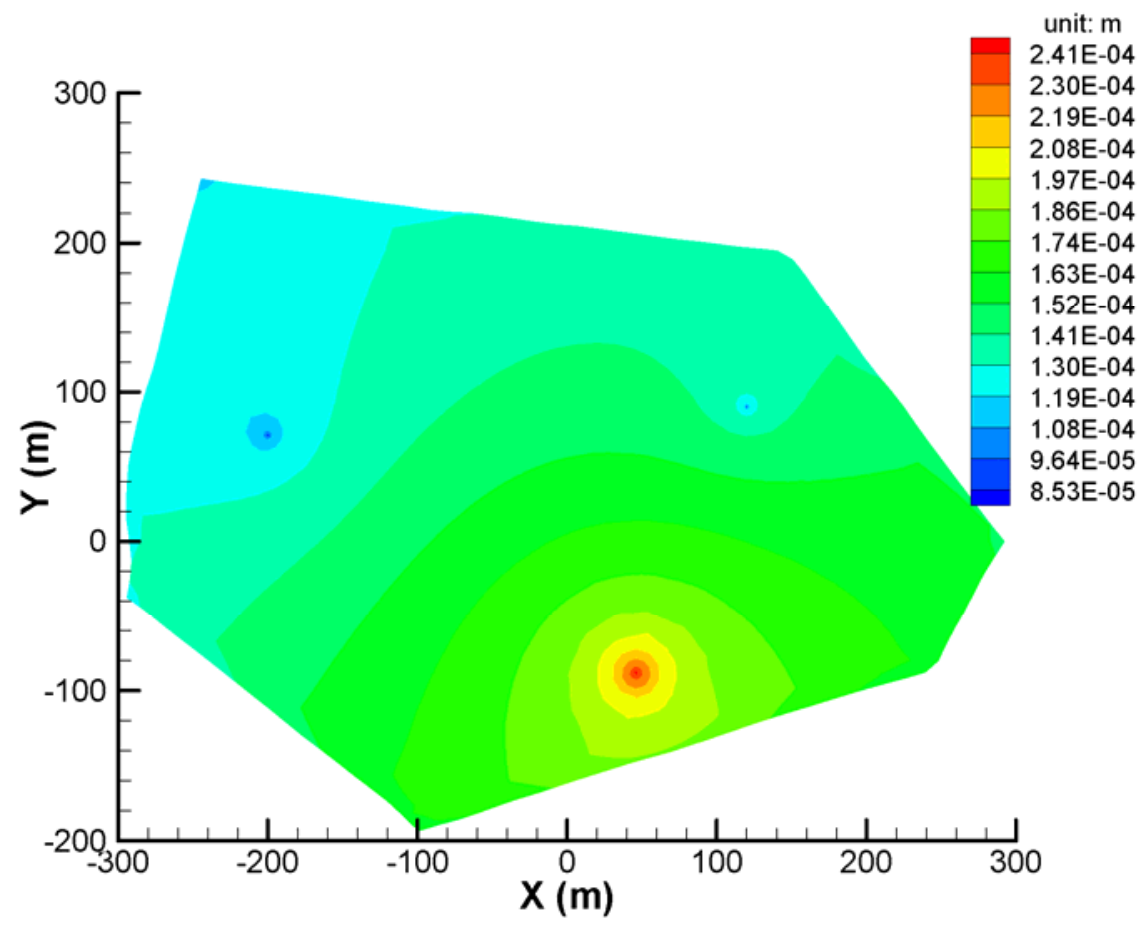

(a) $\mathrm{t}=1.0 \times 10^{5} \mathrm{~s}$

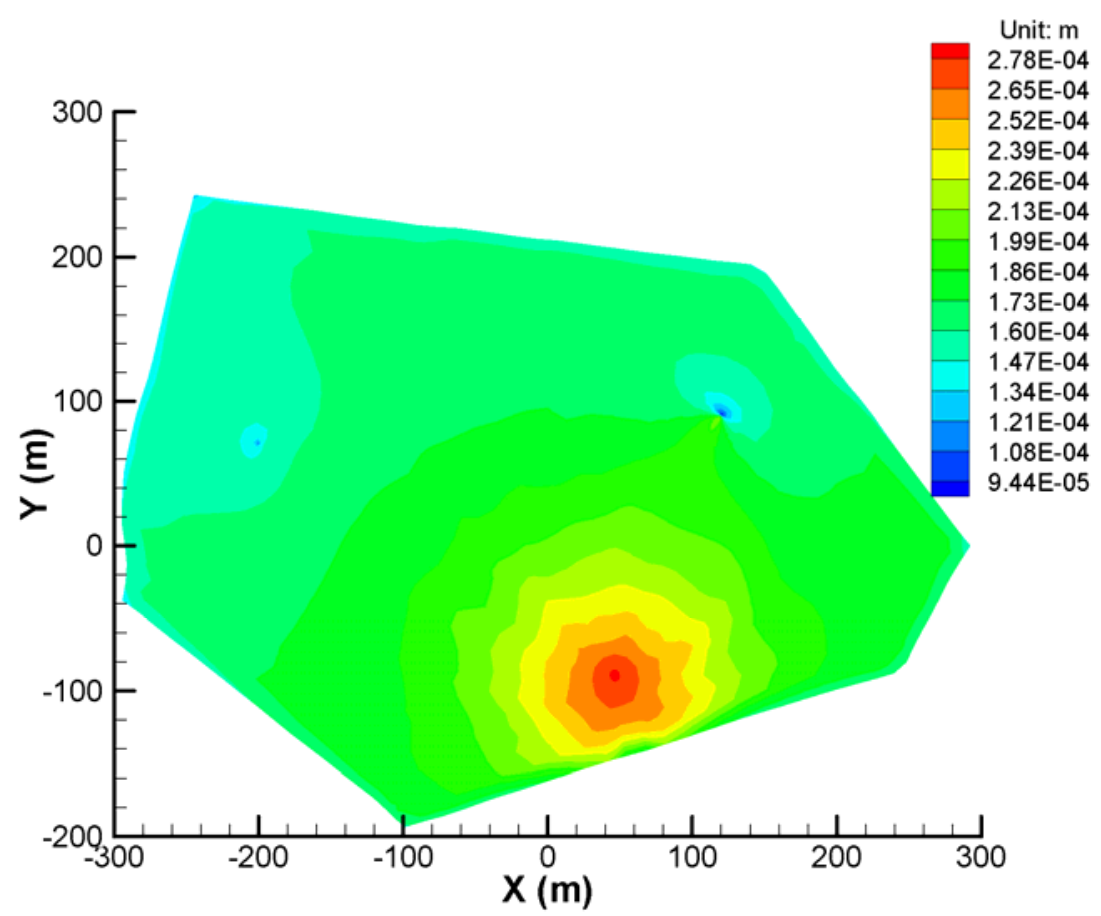

(b) $\mathrm{t}=1.0 \times 10^{7} \mathrm{~s}$ 


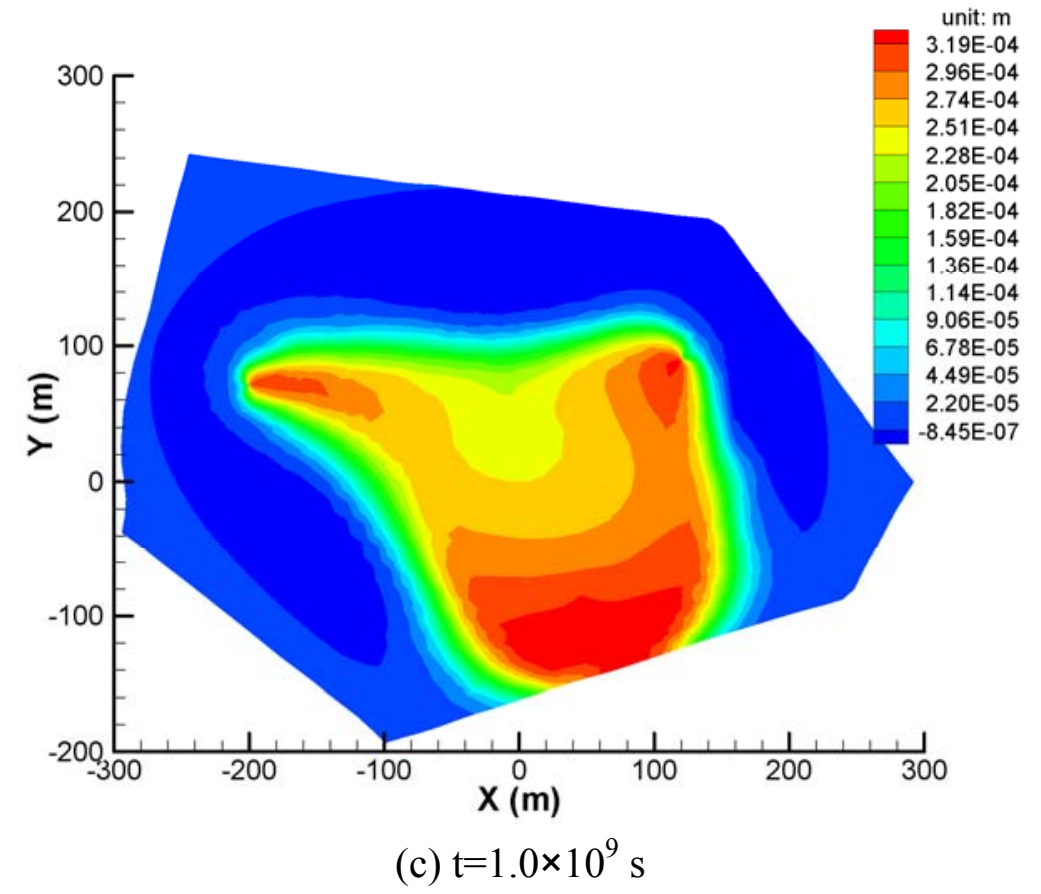

Figure 5.45. Distributions of fracture aperture increment after different fluid injection times: (a) $\mathrm{t}=1.0 \times 10^{5} \mathrm{~s}$; (b) $\mathrm{t}=1.0 \times 10^{7} \mathrm{~s} ;$ (c) $\mathrm{t}=1.0 \times 10^{9} \mathrm{~s}$. 


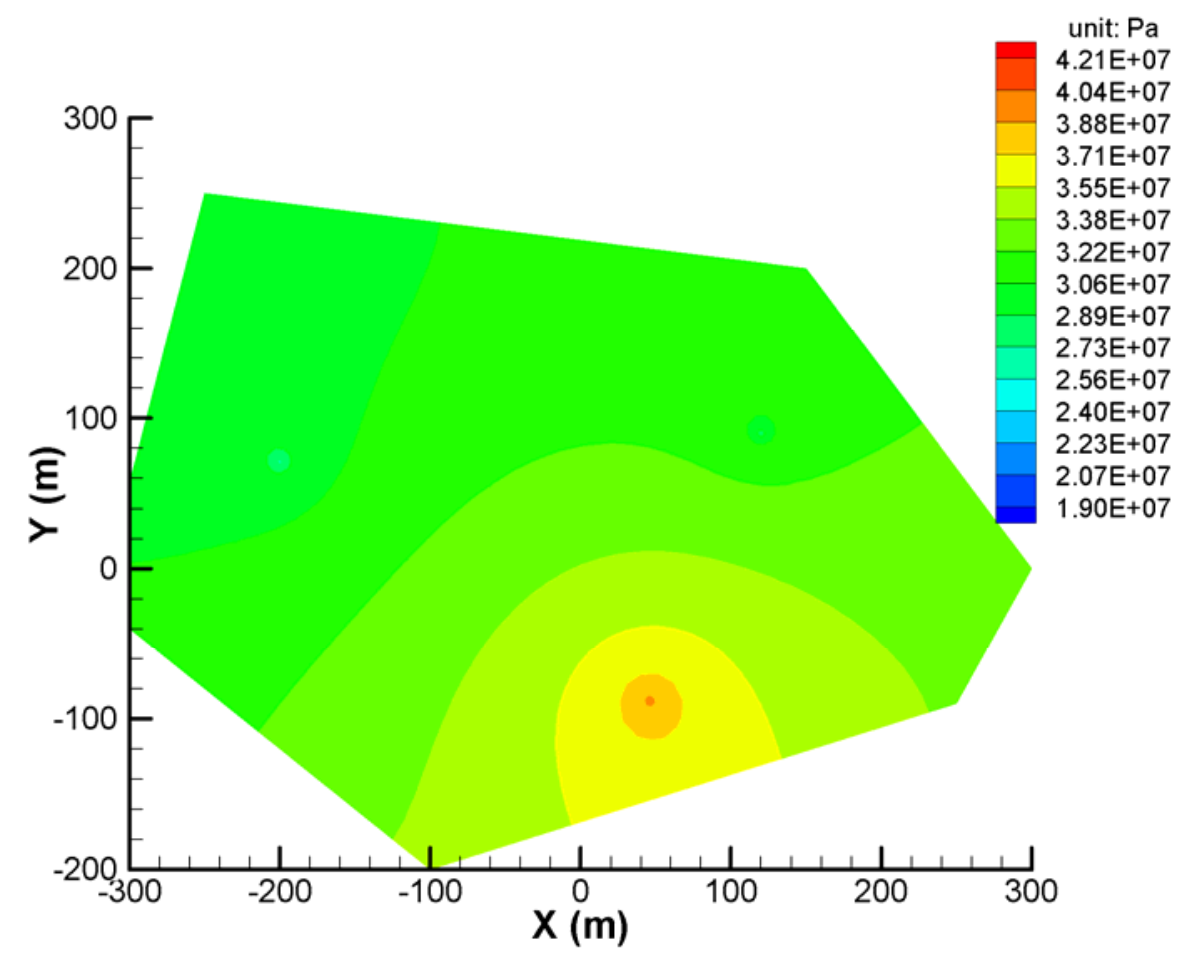

(a) $\mathrm{t}=1.0 \times 10^{5} \mathrm{~s}$

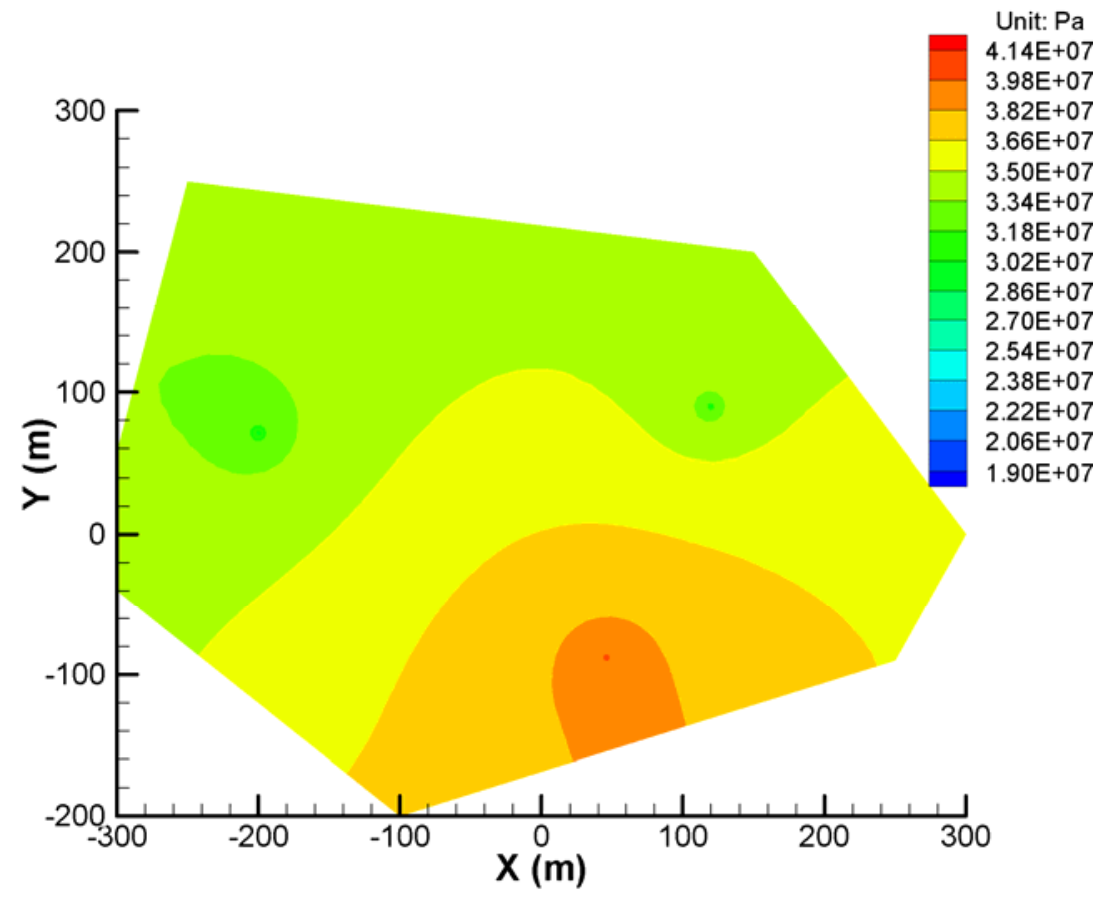

(b) $\mathrm{t}=1.0 \times 10^{7} \mathrm{~s}$ 


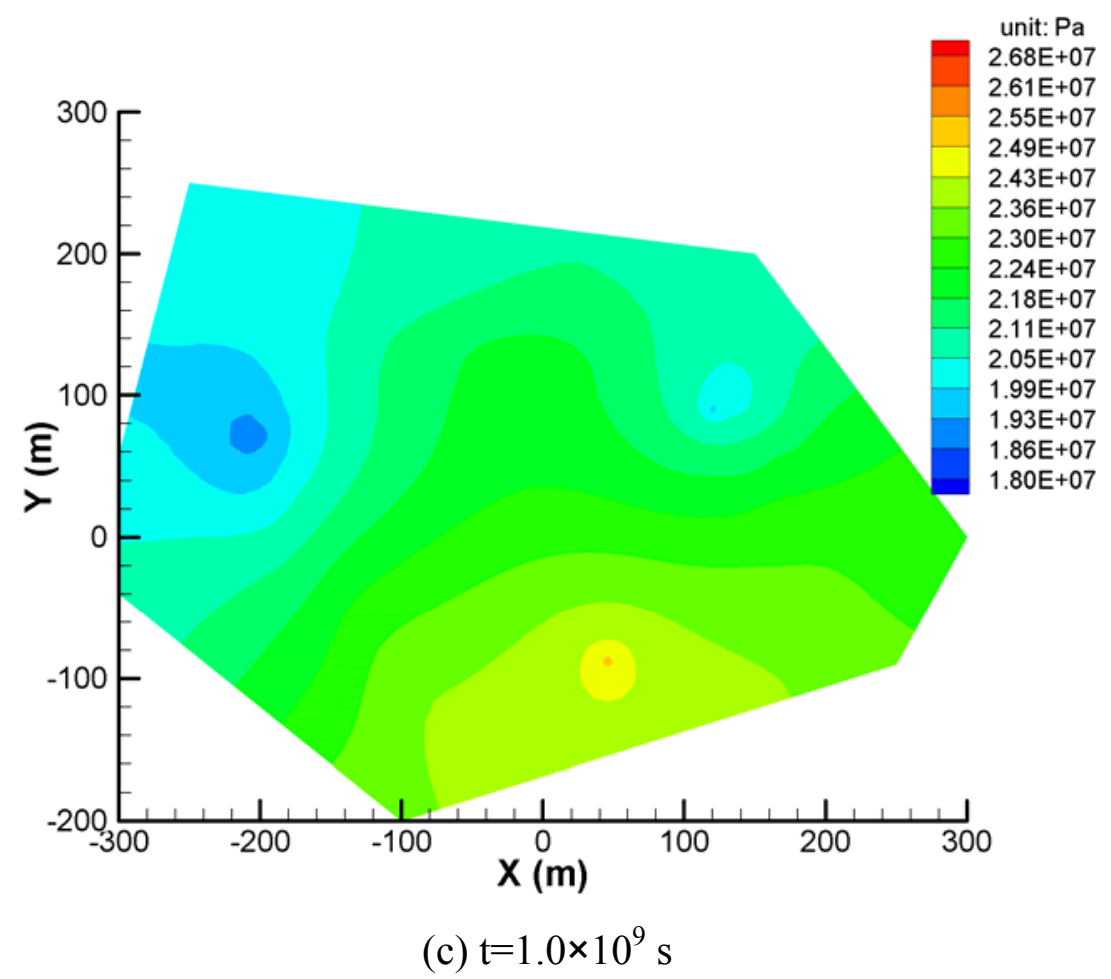

Figure 5.46. Distributions of fluid pressure in the fracture from poro-thermoelastic model after different fluid injection times: (a) $\mathrm{t}=1.0 \times 10^{5} \mathrm{~s}$; (b) $\mathrm{t}=1.0 \times 10^{7} \mathrm{~s}$; (c) $\mathrm{t}=1.0 \times 10^{9} \mathrm{~s}$.

Consistent wit the 2D analysis, the 3D numerical examples clearly show that the fracture pressure and aperture are affected predominantly by the poroelastic effect at early stage of injection, whereas the thermo-effect dominates after a long time of water injection.

When the initial fracture aperture is very small, relatively large fracture pressure is generated compared to the initial reservoir pressure, resulting in a large fluid leakoff that significantly reducers the fracture pressure and aperture compared to the case without fluid leakoff. However, the leakoff influence becomes less evident with the elapse of the fluid injection time when the fracture aperture increases significantly mainly due to the thermo-effect.

The 3D analysis provides a more accurate representation of fracture response and provides means for 3D analysis of slip and stress redistribution in the reservoir. 


\section{REFRENCES}

1. Ghassemi, A., Zhang, Q. 2006. Poro-thermoelastic response of a stationary crack using the displacement discontinuity method. ASCE J. Engineering Mechanics, 132(1), 26-33.

2. Sneddon, I.N., 1946. The distribution of stress in the neighborhood of a crack in an elastic solid. Proc. Royal Soc. London, Ser. A., 187, 229-260.

3. Vandamme, L., E. Detournay., and A.H.-D. Cheng. 1989. A two dimensional poroelastic displacement discontinuity method for hydraulic fracture simulation. Int. J. Numer. Analyt. Meth. Geomech.13: 215-224.

4. Ruina, A.L. 1978. Influence of coupled deformation-diffusion effects on the retardation of hydraulic fracture. In Proc. 19th US Rock Mech. Symp.274-282. University of Nevada Press, Reno, Nevada.

5. Dobroskok, A. A., Ghassemi, A., and Linkov, A.M. 2005. Novozhilov structural criterion: an extension for numerical simulation of mode I and II crack growth and coalescence. Int. J. of Fracture. 133: 223-246.

6. Ghassemi A., Tarasovs S., and Cheng A.H.-D. 2007. A three-dimensional study of the effects of thermo-mechanical loads on fracture slip in enhanced geothermal reservoir. Int. J. Rock Mechanics \& Min Sci., 44, pp. 1132-1148.

7. Segdin, C. M. 1950. Note on a penny-shaped crack under shear. Proc. Camb. Phil. Soc., 47, 396-400.

8. Carter, J.P. and J.R. Booker. 1982. Elastic consolidation around a deep circular tunnel. Int. J. Solids Structures. 18(12), 1059-1074. 


\section{Poro-thermoelastic Analysis of Reactive Flow in a Natural Fracture}

\subsection{Introduction}

As the fluid circulates through the reservoir, it interacts with rocks causing fracture geometry variation in response to chemical as well as mechanical, and thermal processes. Chemical reactions between the rock and the circulating fluid also have been studied and shown to significantly impact the fracture aperture by precipitation and dissolution of minerals [1-3]. Experimental studies [4-6] have shown that chemical precipitation and dissolution of minerals significantly affect fracture aperture [1, 2]. Therefore, it is of interest to understand the poromechanical, thermal and chemical interactions between the fluid and host rock in relation to flow and heat production. Modeling chemical and porothermo-mechanical processes in a fracture-matrix system involves solving equations describing fracture and porous media flow, heat transport, solute transport/reactions and poro-thermoelastic response of the rock and fracture. These mechanisms are generally coupled and occur in an inhomogeneous rock mass that require complex numerical solutions. However, often it is beneficial to conduct a simplified analysis using an analytical solution to a chemical and poro-thermoelastic problem. Furthermore, analytical solutions are useful in testing complex numerical algorithms and also allow one to readily gain insight into the fundamental issues that are involved. In this work, we present a semi-analytical model to solve the non-isothermal reactive flow in a fracture-matrix system considering silica reactivity and poro-thermoelastic effects on fracture aperture. The reactive transport takes into account solute diffusion into the rock matrix and along the fracture. The latter is important because the fracture-matrix coupled system affects the thermal regime as well as the rate at which the concentration gradient between the fracture and the reservoir matrix influences the opening/closure of the fracture aperture (i.e. fracture permeability).

Other investigators have also treated the problem of non-isothermal reactive flow in a fracture both in the context of geological problems and geothermal reservoir development [7-10]. However, previous studies have not coupled chemical, mechanical and thermal mechanisms.

\subsection{Modeling Approach \& Mathematical Model}

For all but the most soluble rocks, the solubility determines the amount of solutes that will be carried along the fracture under large thermal gradient between the fracture and reservoir matrix. A complete description of quartz dissolution and precipitation kinetics is impossible without accurate values for quartz solubility [11]. The reactive modeling of

most minerals is accomplished using the local equilibrium approach without considering their reaction kinetics. Silica deserves a special attention as it equilibrates at a slower rate 
than other minerals and hence, consideration of kinetics becomes inevitable in modeling quartz precipitation/dissolution.

The quartz-water system governed by Eq. 1 is a simple surface reaction to form silicic acid monomer otherwise called as dissolved silica:

$$
2 \mathrm{H}_{2} \mathrm{O}+\mathrm{SiO}_{2} \Leftrightarrow \mathrm{H}_{4} \mathrm{SiO}_{4}
$$

Changes in pressure, in the range occurring in geothermal systems, have much lesser effect on changes in the state of mineral saturation than temperature changes. This is exemplified for quartz, $\mathrm{SiO}_{2}$ [12]; the solubility of which has been well established experimentally over a wide range of temperature and pressure $[12,13]$. The equilibrium solubility of silica and especially quartz is low (amorphous silica 100-140 mg/l, quartz 6$14 \mathrm{mg} / \mathrm{l}$ at earth surface temperature) as described by [14]. As a result, the impact of pressure on solubility is neglected in this work.

Using the above approach, we develop an analytical model to quantify the evolution of fracture aperture and the associated fluid pressure during the dissolution/precipitation of quartz in a granitic rock under laminar flow conditions. The geothermal fluid is injected at the injection well either super-saturated or under saturated with respect to the equilibrium concentration of quartz, through an initially smooth parallel-walled fracture between two blocks of granitic rock.

In general, modeling of the fracture evolution in EGS requires reduction of an extremely complex system to an idealized one based on simple principles. Such an idealized concept has already been used for heat extraction and solute transport. The conceptual model corresponding to an idealized parallel-plate system representing a fracture-matrix coupled system is illustrated in Figure 6.1. The thermal transport mechanisms are thermal advection, thermal conduction and thermal dispersion within the fracture; conductive limited thermal transport from reservoir matrix into the fracture and thermal conduction within the reservoir matrix. The solute transport mechanisms in the fracture are advection, describing the motion of dissolved particles along with the circulating fluid; free diffusion within the fracture, dispersion, limited diffusion from the fracture into the reservoir matrix; dissolution/precipitation of quartz within the fracture and effective diffusion within the reservoir matrix. In this work, matrix diffusion is regarded as a onedimensional process. This assumption is justified if one considers that the solute migration is faster in fractures than in the matrix. The coupling between the fracture and matrix is provided by the continuity of fluxes and concentrations along the interface, assuming that diffusive flux from fracture to matrix takes place perpendicular to the fracture. Diffusion exchanges along the direction parallel to the fracture plane are then negligible as compared with that perpendicular to the fracture plane. Kennedy and Lennox (1995) showed the validity of such an assumption numerically for most cases, except for fractured clay with fracture apertures less than $20 \mu \mathrm{m}$ and flow velocities lower than $1 \mathrm{~m} /$ day. 
In order to incorporate quartz dissolution/precipitation described by linear reaction kinetics, the modified form of fracture and matrix equations is adopted. The radioactive decay term used in the fracture equation is replaced with a mathematically similar linear reaction rate law which describes a first order reaction. This term represents the congruent dissolution/precipitation of quartz described by the temperature dependence of the reaction rate constant and the equilibrium concentration of quartz. The temperature dependence of the equilibrium concentration of quartz is used. In deriving the governing equations for the problem, the following assumptions are made regarding the geometry and hydraulic properties:

(1) the width of the fracture is much smaller than its length

(2) transverse diffusion and dispersion within the fracture assure complete mixing across the fracture width at all times

(3) the permeability of the reservoir matrix is very low and transport within the reservoir matrix occurs mainly by effective molecular diffusion which involves tortuosity

(4) transport along the fracture is much faster than transport within the reservoir

Assumptions 1 and 2 provide the basis for a one-dimensional representation of mass transport along the fracture itself. Assumption 3 and 4 provide the basis for taking the direction of mass flux density in the reservoir matrix to be perpendicular to the fracture axis. The result is the simplification of the two-dimensional system into two coupled orthogonal one-dimensional systems. In addition, the following general assumptions are used in the present study for analyzing the solute transport in a single fracture:

- The fracture and the reservoir matrix are saturated

- The water velocity of the circulating fluid in the fracture is constant

- Quartz dissolution involves a single component system

- Water and rock characteristics, namely longitudinal dispersion coefficient in the fracture, reservoir diffusion coefficient and reservoir porosity do not depend on position

The mathematical model for analyzing silica reactivity and poro-thermoelasticity is formulated based on an idealized system as shown in Figure 6.1. The reservoir is assumed to be horizontal and constrained at top and bottom by rigid, impermeable and thermally insulated formations with constant-thickness (Figure 6.1.a). The fracture is vertical and of uniform width intersecting the entire reservoir thickness.

The plain-strain approximation is used on the horizontal section of the vertical fracture to reduce the solution geometry as shown in Figure 6.1(b). The rock is assumed to be isotropic, homogeneous, and linearly elastic with constant permeability. Fluid flow in the fracture is incompressible, viscous, and laminar such that lubrication flow theory applies and fluid properties are uniform throughout. The fracture aperture is uniform and significantly less than its length. Furthermore, the influence of rock-matrix deformation on pore-pressure is not included as its effect is not major in the current context. We have 
not considered the resistance to opening at the fracture ends and any stiffness that might be offered by a natural fracture.
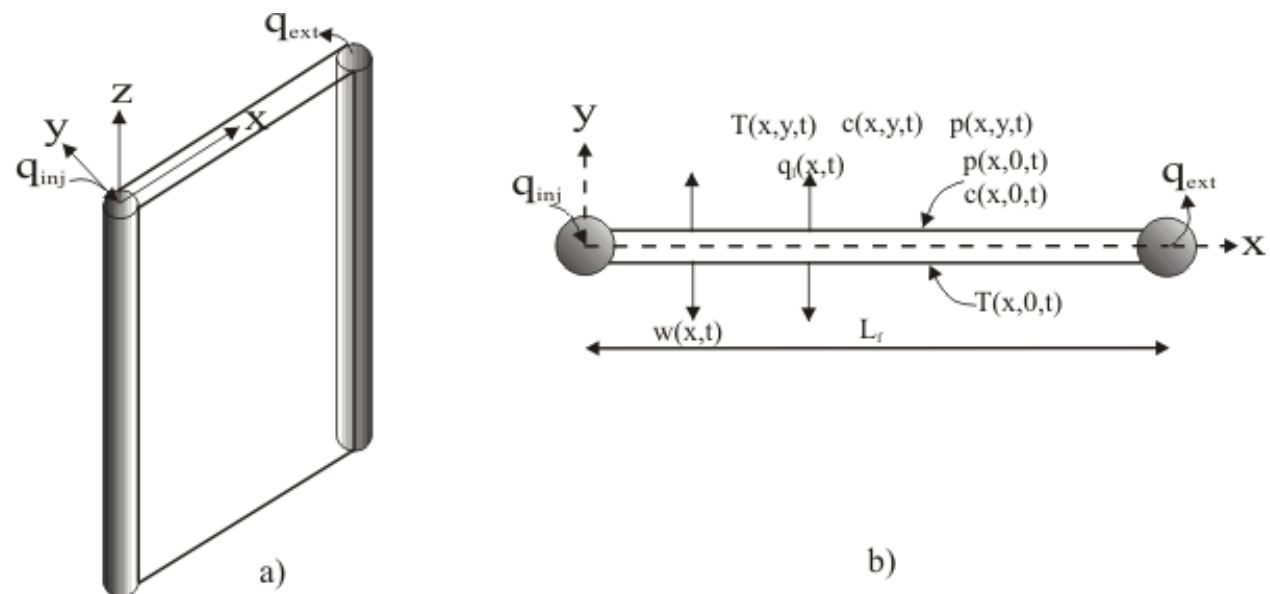

b)

Figure 6.1. Idealized fracture and rock- matrix system.

\subsubsection{Fluid flow in the fracture}

For the lubrication flow theory, the momentum balance indicates that the flow rate is proportional to the pressure gradient as:

$$
\frac{\partial p(x, 0, t)}{\partial x}=-\frac{12 \mu_{f}}{w^{3}(x, t)} q(x, t)
$$

Here $p$ is the pressure in the fracture caused by injection defined as: $p=p(x, 0, t)-p_{o}$ with $p(x, 0, t)$ as the total fracture pressure and $p_{o}$ as the ambient reservoir pressure, $\mu_{f}$ is the fluid viscosity; $w$ is the fracture aperture and $q(x, t)$ is volumetric flow rate per unit height of the vertical fracture: $q(x, t)=w(x, t) v(x, t)$.

Assuming fluid is incompressible and it leaks off from the fracture wall into the formation, the fluid continuity equation is:

$$
\frac{\partial q(x, t)}{\partial x}+2 q_{l}(x, t)=0
$$

where $q_{l}(x, t)$ is the leak-off velocity (positive for loss into the matrix), with the multiplier 2 reflecting the two fracture walls. Now, combining Eqs. (1) and (2) and neglecting $\partial w / \partial x$, a second-order partial differential equation is obtained for fluid pressure in the fracture as shown in below : 


$$
\frac{\partial^{2} p(x, 0, t)}{\partial x^{2}}=\frac{24 \mu_{f}}{w^{3}(x, t)} q_{l}(x, t)
$$

\subsubsection{Fluid flow in the rock-matrix}

In general, the reservoir would be modeled using a fully coupled thermo-poroelasticity to include the coupled interaction between fluid diffusion, heat transport and rock deformation. However, similar to Ghassemi et al. [15], we assume that the coupling between flow process and the elastic deformation is small so that the fluid heat diffusion can be treated separately from the rock deformation. In such case, pore- pressure in the rock- matrix is governed by well-known diffusion equation:

$$
\frac{\partial^{2} p(x, y, t)}{\partial y^{2}}=\frac{1}{c_{D}} \frac{\partial p(x, y, t)}{\partial t} \text { for } y>0
$$

where $c_{D}$ is the fluid diffusivity coefficient . The initial and boundary conditions for Eqs. (1) and (4) are taken as:

$$
p(x, y, 0)=0 \quad p\left(L_{f}, 0, t\right)=0 ;\left.\frac{\partial p(x, 0, t)}{\partial x}\right|_{x=0}=\frac{-12 \mu_{f} q_{o}}{w_{o}^{3}}
$$

where $w_{o}$ is the initial fracture aperture.

\subsubsection{Heat transfer in the fracture and rock-matrix}

Considering heat storage, advection, longitudinal dispersion and conduction from the fracture walls, the heat transport in the fracture can be expressed as:

$$
\frac{\partial T(x, 0, t)}{\partial t}+v(x, t) \frac{\partial T(x, 0, t)}{\partial x}-D_{f} \frac{\partial^{2} T(x, 0, t)}{\partial x^{2}}-D_{T} \frac{\partial^{2} T(x, 0, t)}{\partial x^{2}}=\left.\frac{2 \lambda_{m m}}{\rho_{f} c_{w} w(x, t)} \frac{\partial T(x, y, t)}{\partial y}\right|_{y=0}
$$

Studies on the heat transfer suggest conduction and dispersion effect within the fracture are small, hence can be ignored [16]. With these simplifications Eq. (6) becomes:

$$
\frac{\partial T(x, 0, t)}{\partial t}+v(x, t) \frac{\partial T(x, 0, t)}{\partial x}=\left.\frac{2 \lambda_{m m}}{\rho_{f} c_{w} w(x, t)} \frac{\partial T(x, y, t)}{\partial y}\right|_{y=0}
$$

Assuming the heat conduction in the rock-matrix to be one-dimensional perpendicular to fracture, and considering the presence of leak-off, the heat transport in the rock-matrix is governed by:

$$
\frac{\partial T(x, y, t)}{\partial t}=\frac{\lambda_{m m}}{\rho_{m} c_{r}} \frac{\partial^{2} T(x, y, t)}{\partial y^{2}}-q_{l}(x, t) \frac{\partial T(x, y, t)}{\partial y}
$$


The initial and boundary conditions associated with Eqs. (7)-(8) are:

$$
\begin{aligned}
& T(x, y, 0)=T_{r o} \\
& T(0,0, t)=T_{f o}
\end{aligned}
$$

where $T_{r o}$ is the initial rock temperature and $T_{f o}$ is the fluid temperature at the injection point.

\subsubsection{Solute transport and reactivity}

The issue of mineral dissolution and precipitation in geothermal systems can be complex. But, when studying silica reactivity, using a single component reactive transport model is adequate under conditions of interest [17-20]. In the present work, the principal solute transport mechanisms in a fracture are considered to be: solute advection, diffusionlimited mass solute transfer from the fracture into the rock-matrix; dissolution and precipitation of silica within the fracture, and diffusive transport within the rock-matrix.

By assuming one-dimensional mass transport along the fracture and considering the matrix mass-flux to be perpendicular to the fracture plane, the two-dimensional system is simplified into two coupled orthogonal one-dimensional systems. In addition, we assume that fluid and rock characteristics e.g., rock-matrix diffusion coefficient and rock-matrix porosity do not depend on the spatial position. The resulting single-component solute transport system incorporates the silica dissolution/precipitation using linear reaction kinetics. As an extension, we consider the impact of fluid leak-off and temperaturedependent reaction rate and the equilibrium concentration within the fracture and the rock-matrix [3]. By considering reaction rates and equilibrium concentration as function of temperature, a non-linearity (of variable coefficients) is introduced in second order partial differential equation system of solute transport, for example:

$$
\begin{aligned}
& \frac{\partial c^{\prime}(x, y, t)}{\partial t}=D^{\prime} \frac{\partial^{2} c^{\prime}(x, y, t)}{\partial y^{2}}-\frac{k_{m}[f(T(x, y, t)]}{\phi} c^{\prime}(x, y, t)-q_{l}(x, t) \frac{\partial c^{\prime}(x, y, t)}{\partial y} \\
& \frac{\partial c^{\prime}(x, 0, t)}{\partial t}=-v(x, t) \frac{\partial c^{\prime}(x, 0, t)}{\partial x}-k_{f}\left[f(T(x, y, t)] c^{\prime}(x, 0, t)+\left.\frac{2 \phi D^{\prime}}{w(x, t)} \frac{\partial c^{\prime}(x, y, t)}{\partial y}\right|_{y=0}\right.
\end{aligned}
$$

To overcome the difficulty in deriving analytical solutions for Eqs. (11) and (12), the solute transport is decoupled from thermal transport, such that variable coefficients in solute transport are only functions of temperature and these are computed after all temperature field calculations are completed. The temperature dependent reaction rate is adopted from [21] and equilibrium concentration of quartz is obtained from [11], while the expressions for the effective reaction rate constants are adopted from Steefel et al. [18] (see details in section 6.3.1). So, the governing solute transport equations for the case of linear reaction kinetics and with fluid leak-off can be simplified as: 


$$
\begin{aligned}
& \frac{\partial c^{\prime}(x, y, t)}{\partial t}=D^{\prime} \frac{\partial^{2} c^{\prime}(x, y, t)}{\partial y^{2}}-\frac{k_{m}}{\phi} c^{\prime}(x, y, t)-q_{l}(x, t) \frac{\partial c^{\prime}(x, y, t)}{\partial y} \\
& \frac{\partial c^{\prime}(x, 0, t)}{\partial t}=-v(x, t) \frac{\partial c^{\prime}(x, 0, t)}{\partial x}-k_{f} c^{\prime}(x, 0, t)+\left.\frac{2 \phi D^{\prime}}{w(x, t)} \frac{\partial c^{\prime}(x, y, t)}{\partial y}\right|_{y=0}
\end{aligned}
$$

In Eqs.(13) and (14),$c^{\prime}(x, y, t)$ and $c^{\prime}(x, 0, t)$ are defined as:

$$
\begin{aligned}
& c^{\prime}(x, y, t)=c(x, y, t)-c^{e q}(x, y, t) \\
& c^{\prime}(x, 0, t)=c(x, 0, t)-c^{e q}(x, 0, t)
\end{aligned}
$$

The Eqs. (14) and (15) are subjected to the initial and boundary conditions; it is assumed that initially, before fluid injection/production begins, the fluid in the fracture is at equilibrium concentration. Similarly, at the injection well, a constant concentration in the injected fluid exists. So they are:

$$
\begin{aligned}
& c^{\prime}(x, y, 0)=0 \\
& c^{\prime}(0,0, t)=c_{o}^{\prime}
\end{aligned}
$$

where $c_{o}^{\prime}$ source concentration above the equilibrium level.

\subsection{Fracture aperture change due to silica dissolution and precipitation and poro-thermoelastic effects}

The fracture aperture can change due to silica dissolution/precipitation and porothermoelastic deformation caused by fluid pressure in the fracture thus affecting the conductivity and fluid flow. These mechanisms of fracture aperture change are described in detail in following:

\subsubsection{Silica dissolution and precipitation and aperture variation}

Considering quartz as the main mineral which affects the fracture aperture in dissolution and precipitation processes, its reactivity of quartz can be modeled using a rate law $[11,21]$ :

$$
\frac{d c}{d t}=K_{r} a^{*}\left(c^{e q}-c\right)
$$

The intrinsic rate constant, $K_{r}$ and the equilibrium concentration of quartz are assumed to be function of temperature alone [11,21] as the pressure does not play an important role because the magnitude of silica concentration change with pressure is 
generally significant order of magnitude less than its temperature counterpart and is given as:

$$
\begin{aligned}
& K_{r}(T)=10^{0.433-4090 / T} \\
& c^{e q}(T)=6 \times 10^{4} \times 10^{1.881-2.028 \times 10^{-3} T-1560 / T}
\end{aligned}
$$

The effective reaction rate constants in the fracture and rock-matrix are computed as:

$$
k_{f}=\frac{2 K(T)}{w_{o}} ; k_{m}=A_{m} K(T)
$$

where $A_{m}$ is mineral surface area [18].

In addition to computing the concentration profile within the fracture at various times, the cumulative mass of silica dissolved from or deposited on the rock surfaces at each position along the fracture is examined. For a given time interval $\Delta t$, the mass of silica dissolved or deposited per unit fracture length, $m_{q}$ is given by [21]:

$$
m_{q}=\frac{10^{-6} \rho_{f} \Delta t V_{f} K_{r} a^{*}\left(c^{e q}-c\right)}{L_{f}}
$$

where $a^{*}\left(=2 f_{q} / w_{o}\right)$ is the surface area to fluid volume ratio and $f_{q}$ is the volume fraction of quartz in the rock matrix. The quantity of quartz/amorphous silica dissolved or deposited can be related to the fractional change in the average fracture aperture by assuming fracture flow geometry in Figure 6.1. The fractional aperture change $(\Delta \omega)$ can be described as the volume change of quartz/amorphous silica divided by the fluid volume and is given by Robinson and Pendergrass [21]

$$
\Delta \omega=-\frac{L_{f} m_{q}}{\rho_{q} V_{f}}=-\frac{10^{-6} \rho_{f} \Delta t K_{r} a^{*}\left(c^{e q}-c\right)}{\rho_{q}}
$$

The fractional aperture change due to silica dissolution and precipitation is related to the induced fracture aperture as $\Delta \omega=1-w(x, t) / w_{o}$. Using this expression into Eq.(23), the induced fracture aperture $w(x, t)$ due to silica dissolution and precipitation can be written as:

$$
w(x, t)=w_{o}\left[1+\frac{10^{-6} \rho_{f} \Delta t K_{r} a^{*}\left(c^{e q}-c\right)}{\rho_{q}}\right]
$$




\subsubsection{Aperture variation caused by poro-thermoelasticity}

The fracture aperture can change due to the elastic deformation exerted by the pressure in the fracture. It can also be affected by the thermal and pore pressure diffusion into the formation. The 3D field equations considering these coupled effects are (Ghassemi et al. [26]:

$$
\begin{aligned}
& G \frac{\partial^{2} u_{i}}{\partial x_{i} \partial x_{i}}+\left(\frac{G}{1-2 v}\right) \frac{\partial^{2} u_{j}}{\partial x_{j} x_{i}}-\alpha \frac{\partial p}{\partial x_{i}}-\beta_{s} K \frac{\partial T}{\partial x_{i}}=0 \\
& \frac{1}{M} \frac{\partial p}{\partial t}=\frac{k}{\mu} \frac{\partial^{2} p}{\partial x_{i} \partial x_{i}}-\alpha \frac{\partial}{\partial t}\left(\frac{\partial u_{i}}{\partial x_{i}}\right)+d \frac{\partial T}{\partial t} \\
& \frac{\partial T}{\partial t}=\frac{\lambda_{m m}}{\rho_{m} c_{r}} \frac{\partial^{2} T}{\partial x_{i} \partial x_{i}}-q_{l} \frac{\partial T}{\partial x_{i}}
\end{aligned}
$$

with $\quad M=\frac{2 G\left(v_{u}-v\right)}{\alpha^{2}(1-2 v)\left(1-2 v_{u}\right)}$, and where $\mathrm{d}=\alpha \beta_{s}+\phi\left(\beta_{f}-\beta_{s}\right) ; \kappa=k / \mu ; G$ is shear modulus and $\mathrm{M}$ is Biot's modulus.

For most rocks, heating/cooling produces thermal stresses and affects pore pressure, but stress and pressure changes do not significantly alter the temperature field so that the latter is not coupled to the equations for pore pressure and solid displacements. Also, note that convective heat transport is neglected. This is justified for rocks of interest, i.e. rocks that are impermeable or have low matrix permeability [26].

The system of Eq. (25) needs to be solved simultaneously together with boundary conditions on the fracture surface. The boundary conditions are temperature and pressure in the fracture, which are also unknown as they are governed by the fracture flow and heat transport equations. The solution of this system yields the information about fracture opening resulting from the combined elastic, pore pressure, and temperature effect as $\mathrm{w}(\mathrm{x}, \mathrm{t})=2 \mathrm{u}_{\mathrm{y}}(\mathrm{x}, 0, \mathrm{t})$.

In order to solve the above sub-problem analytically, we assume the solid displacement to be one dimensional and perpendicular to the fracture surface (in the $y$-direction). In view of this assumption, Eq. (25).a becomes:

$$
\frac{\partial^{2} u_{y}(x, y, t)}{\partial y^{2}}=\chi \frac{\partial T(x, y, t)}{\partial y}+\frac{\eta}{G} \frac{\partial p(x, y, t)}{\partial y}
$$

where $\chi=\frac{\alpha_{T}(1+v)}{1-v}, \eta=\frac{\alpha(1-2 v)}{2(1-v)}$, and $\alpha$ is Biot's effective stress coefficient. $\alpha_{T}$ is the linear thermal expansion coefficient of the rock, $\left(\beta_{s} / 3\right)$. Eqn. (26) is integrated twice from the fracture surface $(\mathrm{y}=0)$ to infinity, assuming $\left.u_{y}\right|_{y=\infty}=0$ : 


$$
-u_{y}(x, 0, t)=\chi \int_{0}^{\infty} \Delta T(x, y, t) d y+\frac{\eta}{G} \int_{0}^{\infty} \Delta p(x, y, t) d y
$$

where:

$$
\begin{aligned}
& \Delta T(x, y, t)=T(x, y, t)-T(x, \infty, t) \\
& \Delta p(x, y, t)=p(x, y, t)-p(x, \infty, t)
\end{aligned}
$$

With $T(x, \infty, t)=T_{r o}$ and $p(x, \infty, t)=p_{o}$, where $p_{o}$ is the in-situ reservoir pressure, and differentiating Eq. (27) with respect to time one gets:

$$
-\frac{\partial u_{y}(x, 0, t)}{\partial t}=\chi \int_{0}^{\infty} \frac{\partial \Delta T(x, y, t)}{\partial t} d y+\frac{\eta}{G} \int_{0}^{\infty} \frac{\partial \Delta p(x, y, t)}{\partial t} d y
$$

\subsection{Solution of Temperature, Concentration, Pore Pressure and Aperture with Constant Leak-off into Permeable Rock-Matrix}

To be able to solve equation of poro-thermo-elasticity and solute transport and its reactivity semi-analytically, we relax the condition of fluid leak-off into the formation by treating it as constant along the fracture face (i.e. fluid leaking off into the formation is constant in magnitude). By assuming this, we first solve for the pressure in the fracture and rock-matrix. Then solving for temperature and fracture aperture change due to thermoelasticity is followed by solute transport and its effect on fracture aperture. Similarly poroelastic effects i.e. change in fracture aperture due to pore pressure are computed.

For the constant leak-off, $q_{l o}$ and constant injection rate, $q_{o}$ the integration of the continuity Eq. (2) gives the flow rate as:

$$
q(x)=q_{o}-2 q_{l o} x
$$

Here $q_{l o}=m q_{o} / 2 L_{f}$ and $m$ is a fluid loss coefficient, can be defined as the ratio between fluid loss and the injection rate [16]. Substituting Eq. (30) into Eq. (1) yields:

$$
\frac{\partial p(x, 0, t)}{\partial x}=-\frac{12 \mu_{f}}{w^{3}(x, t)}\left[q_{o}-2 q_{l o} x\right]
$$

Integrating Eq. (31) with respect to $\mathrm{x}$ and applying boundary condition in Eq. (5) it follows:

$$
p(x, 0, t)=12 \mu_{f} \int_{x}^{L_{f}} \frac{\left(q_{o}-2 q_{l o} \varsigma\right)}{w^{3}(\varsigma)} d \varsigma
$$


Fluid diffusion Eq. (4) can be solved by applying Laplace transformation and utilizing the boundary condition in Eq. (5) as:

$$
\frac{\partial^{2} \tilde{p}(x, y, s)}{\partial y^{2}}=\frac{s}{c_{D}} \tilde{p}(x, y, s)
$$

The solution for induced pressure in time domain with substitution of Eq. (32) leads to:

$$
p(x, y, t)=12 \mu_{f} \operatorname{erfc}\left(\frac{y}{2 \sqrt{c_{D} t}}\right) \int_{x}^{L_{f}} \frac{\left(q_{o}-2 q_{l o} \varsigma\right)}{w^{3}(\varsigma)} d \varsigma
$$

\subsubsection{Temperature solution and thermoelastic effects on fracture aperture}

For the constant leak-off as noted in Eq.(30), the solution of Eqs. (7)-(10) in Laplace space is [e.g., 16] is:

$$
\tilde{T}_{D}(x, y, s)=\tilde{T}_{D}(x, 0, s) \exp \left[\frac{\rho_{m} c_{r} q_{l o} \lambda_{T-}}{2 \lambda_{m m}} y\right]
$$

with

$$
\begin{aligned}
& \tilde{T}_{D}(x, 0, s)=\frac{1}{s} \exp \left[-\frac{s}{v} x-\frac{\rho_{m} c_{r} \lambda_{T-}}{2 \rho_{f} c_{w}} \log \left(1-\frac{2 q_{l o}}{q_{o}} x\right)\right] \\
& \text { where } \lambda_{T-}=1-\sqrt{1+\frac{4 \lambda_{m m} s}{\rho_{m} c_{r} q_{l o}^{2}}} \text { and } T_{D}=\frac{T_{r o}-T(x, y, t)}{T_{r o}-T_{f o}}
\end{aligned}
$$

To get the fracture aperture change due to thermoelasticity, considering only the effects of thermoelasticity in the governing equation of poro-thermo-elasticity (Eqn. (29) as:

$$
\frac{\partial u_{y}(x, 0, t)}{\partial t}=\frac{1}{2} \frac{\partial w(x, t)}{\partial t}=-\chi \frac{d}{d t}\left(\int_{0}^{\infty} \Delta T(x, y, t) d y\right)
$$

where $w(x, t)=2 u_{y}(x, 0, t)$. Substituting Eq. (8) with Eq. (28) into Eq. (37) it yields:

$$
\frac{\partial w(x, t)}{\partial t}=\left.2 \chi \frac{\lambda_{m m}}{\rho_{m} c_{r}} \frac{\partial \Delta T(x, y, t)}{\partial y}\right|_{y=0}-2 \chi q_{l o} \Delta T(x, 0, t)
$$

Taking Laplace transform of Eqn. (38) and with knowing that $\Delta T(x, y, t)=T(x, y, t)-T_{r o}$, it yields: 


$$
\tilde{w}(x, s)=\frac{w_{o}}{s}-\left.2 T^{\Delta} \chi \frac{\lambda_{m m}}{s \rho_{m} c_{r}} \frac{\partial \tilde{T}_{D}(x, y, s)}{\partial y}\right|_{y=0}+\frac{2 T^{\Delta} \chi q_{l 0}}{s} \tilde{T}_{D}(x, 0, s)
$$

The gradient term in Eq. (39) is obtained from Eq. (35), as :

$$
\left.\frac{\partial \tilde{T}_{D}(x, y, s)}{\partial y}\right|_{y=0}=\tilde{T}_{D}(x, 0, s) \frac{\rho_{m} c_{r} q_{l o} \lambda_{T-}}{2 \lambda_{m m}}
$$

Substituting Eq. (40) into (39) and simplifying it yields

$$
\tilde{w}(x, s)=\frac{w_{o}}{s}+\frac{T^{\Delta} \chi q_{l o}\left(2-\lambda_{T-}\right)}{s} \tilde{T}_{D}(x, 0, s)
$$

Combining Eqs. (36) and (41) leads to:

$$
\tilde{w}(x, s)=\frac{w_{o}}{s}+\frac{T^{\Delta} \chi q_{l o}\left(2-\lambda_{T-}\right)}{s^{2}} \exp \left[-\frac{s}{v} x-\frac{\rho_{m} c_{r} \lambda_{T-}}{2 \rho_{f} c_{w}} \log \left(1-\frac{2 q_{l o}}{q_{o}} x\right)\right]
$$

where $T^{\Delta}=T_{r o}-T_{f o}$ and initial fracture aperture i.e. $w(x, 0)=w_{o}$. The solution must be numerically inverted in time domain which is done using Stehfast [24] method.

\subsubsection{Solution for silica concentration}

Considering fluid leak-off, the solution of Eqs. (13)-(17) for the concentration in the rock-matrix and the fracture in Laplace space are given as:

$$
\tilde{c}^{\prime}(x, y, s)=\tilde{c}^{\prime}(x, 0, s) \exp \left[\frac{q_{l o} \lambda_{C-}}{2 D^{\prime}} y\right]
$$

with

$$
\tilde{c}^{\prime}(x, 0, s)=\frac{c_{o}^{\prime}}{s} \exp \left[-\frac{\left(k_{f}+s\right)}{v} x-\frac{\phi \lambda_{C-}}{2} \log \left(1-2 \frac{q_{l o}}{q_{0}} x\right)\right]
$$

where

$$
\lambda_{C-}=1-\sqrt{1+\frac{4 D^{\prime}}{q_{l o}^{2}}\left(\frac{k_{m}}{\phi}+s\right)}
$$

Thus the fracture aperture due to silica dissolution and precipitation can now be calculated using the concentration in the fracture is computed from Eq. (44) and substituting it into Eq. (24) with other known and computed variables (e.g. effective reaction rate constant). 


\subsubsection{Pore pressure solution and poroelastic effects on fracture aperture}

Ignoring the thermoelastic effects for the time being and considering poroelastic effects only Eq. (29) is thus leads to:

$$
\frac{\partial u_{y}(x, 0, t)}{\partial t}=\frac{1}{2} \frac{\partial w(x, t)}{\partial t}=-\frac{\eta}{G} \frac{d}{d t}\left(\int_{0}^{\infty} \partial \Delta p(x, y, t) d y\right)
$$

Simplifying Eqn. (45) using governing Eq. (4) as shown in below

$$
\frac{\partial w(x, t)}{\partial t}=\left.\frac{2 \eta c_{D}}{G} \frac{\partial \Delta p(x, y, t)}{\partial y}\right|_{y=0}
$$

Now realizing $\Delta p(x, y, t)=p(x, y, t)$ from Eq. (5) and differentiating pressure Eq. (34) w. r. t. y and combining it with Eq. (46), it leads to:

$$
\frac{\partial w(x, t)}{\partial t}=-\frac{2 \eta \sqrt{c_{D}} \Delta p(x, 0, t)}{G \sqrt{\pi t}}
$$

where $\Delta p(x, 0, t)=p_{\text {imp }}(x, 0, t)-p_{\text {perm }}(x, 0, t)$ is the pressure difference between the permeable and impermeable cases. Simplifying for the permeable and impermeable cases yields: $\Delta p(x, 0, t)=24 \mu_{f} q_{l o} \int_{x}^{L_{f}} \frac{\varsigma}{w^{3}(\varsigma)} d \varsigma$. Substituting this expression into Eq. (47) and integrating $\mathrm{w}$. r. t. time knowing initial condition as $\mathrm{w}(\mathrm{x}, 0)=\mathrm{w}_{\mathrm{o}}$ yields:

$$
w(x, t)=w_{o}-\frac{96 \eta \mu_{f} q_{l o}}{G} \sqrt{\frac{c_{D} t}{\pi}} \int_{x}^{L_{f}} \frac{\varsigma}{w^{3}(\varsigma)} d \varsigma
$$

\subsection{Solution for Temperature, Concentration \& Aperture for Impermeable Rock}

Impermeable solutions play important role if the reservoir can be treated as impermeable with respect to the fluid leak-off from the fracture into the rock-matrix. Moreover, these solutions can be readily solved and can be used to validate e.g. bound solution (no leakoff) of complex numerical algorithm. The impermeable solutions for chemo-poro-thermo model can be found by neglecting leak-off into the formation i.e. $q_{l}(x, t)=0$ in the governing equations. However, for this case poroelastic effects are not considered here. The impermeable solution for temperature can be calculated as (e.g. in Gringarten et al. [25]: 


$$
T_{D}(x, 0, t)= \begin{cases}\operatorname{erfc}\left[\frac{\lambda_{m m} x}{q_{o} \rho_{f} c_{w}} \sqrt{\frac{v \rho_{m} c_{r}}{\lambda_{m m}(v t-x)}}\right] & \text { for } x \leq v t \\ 0 & \text { for } x>v t\end{cases}
$$

Substituting Eq. (49) into Eq. (29) with considering only thermoelastic effects, the fracture aperture change due to thermoelastic can be given as:

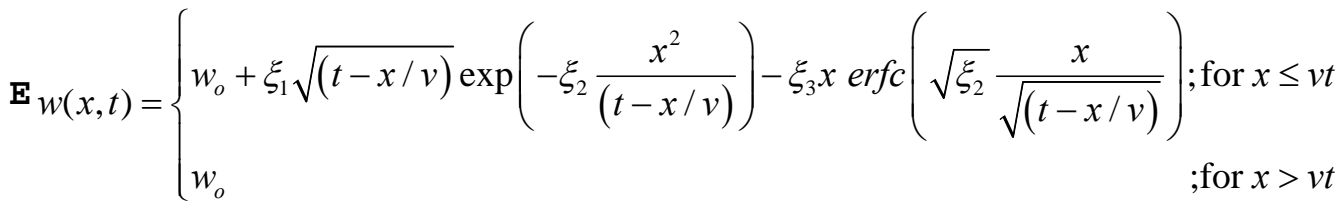

where $\xi_{1}=\frac{4 \chi T^{\Delta} \sqrt{\lambda_{m m}}}{\sqrt{\pi \rho_{m} c_{r}}} ; \xi_{2}=\frac{\lambda_{m m} \rho_{m} c_{r}}{\left(q_{o} \rho_{f} c_{w}\right)^{2}}$ and $\xi_{3}=\frac{4 \chi T^{\Delta} \lambda_{m m}}{q_{o} \rho_{f} c_{w}}$

Similarly, neglecting leak-off into the formation and assuming constant velocity in the fracture, the solution for solute concentration (Eqs. (13)-(17) ) in time domain is (e.g. in (Steefel et al., 1998) :

$$
\begin{aligned}
& c^{\prime}(x, 0, t)=0, \text { if } \mathrm{t}<\frac{x}{v} \\
& c^{\prime}(x, 0, t)=\frac{c_{o}^{\prime}}{2}\left\{\exp \left(-\frac{x}{\lambda_{f}}\right) \operatorname{erfc}\left(\xi_{+}\right)+\exp \left[-x\left(\frac{1}{\lambda_{f}^{0}}-\frac{1}{\lambda_{m} P_{e}}\right)\right] \operatorname{erf} c\left(\xi_{-}\right)\right\}, \text {if } \mathrm{t}>\frac{x}{v}
\end{aligned}
$$

where

$$
\begin{aligned}
& \xi_{ \pm}=\frac{x}{2 P_{e} \sqrt{D^{\prime}\left(t-\frac{x}{v}\right)}} \mp \frac{1}{\lambda_{m}} \sqrt{D^{\prime}\left(t-\frac{x}{v}\right)} \\
& P_{e}=\frac{v w_{o}}{2 \phi D^{\prime}} ; \lambda_{m}=\sqrt{\frac{\phi D^{\prime}}{k_{m}}} ; \lambda_{f}=\frac{1}{\frac{1}{\lambda_{f}^{0}}+\frac{1}{P e \lambda_{m}}} ; \lambda_{f}^{0}=\frac{v}{k_{f}} \geq \lambda_{f}
\end{aligned}
$$

Similarly, solution of concentration in the rock-matrix in time domain can be solved and given as:

$$
\begin{aligned}
& c^{\prime}(x, y, t)=0, \text { if }\left(t<\frac{x}{v}\right) \\
& c^{\prime}(x, y, t)=\frac{c_{o}^{\prime}}{2}\left\{\exp \left[-\left(\frac{x}{\lambda_{f}}+\frac{y}{\lambda_{m}}\right)\right] \operatorname{erfc}\left(\eta_{+}\right)+\exp \left[-\left(\frac{x}{\lambda_{f}^{0}}-\frac{x}{P e \lambda_{m}}-\frac{y}{\lambda_{m}}\right)\right] \operatorname{erfc}\left(\eta_{-}\right)\right\}, \text {if }\left(t>\frac{x}{v}\right)
\end{aligned}
$$


where, $\eta_{ \pm}=\frac{\frac{x}{P_{e}}+y}{2 \sqrt{D^{\prime}\left(t-\frac{x}{v}\right)}} \mp \frac{1}{\lambda_{m}} \sqrt{D^{\prime}\left(t-\frac{x}{v}\right)}$

\subsection{Numerical Examples}

Silica dissolution or precipitation occurs when the injectate is either under- or supersaturated. The amount of mass transfer is governed by the kinetics of the reaction. When the injectate is pure water, silica in the rock-matrix get dissolved and thus fracture aperture is increased (Figure 6.2). Fracture aperture along the fracture is amplified as the injection of fluid continues which can be attributed to falling of concentration along the fracture as shown in Figure 6.2 .

Table 5. Input parameters used in the example case

\begin{tabular}{lll}
\hline Parameter & Value & Units \\
\hline$q_{o}$ & $1 \times 10^{-4}$ & $\mathrm{~m}^{2} \mathrm{~s}^{-1}$ \\
$w_{o}$ & 0.001 & $\mathrm{~m}$ \\
$\phi$ & 0.01 & - \\
$v$ & 0.25 & - \\
$\alpha_{T}$ & $8.0 \times 10^{-6}$ & $\mathrm{~K}^{-1}$ \\
$\rho_{m}$ & 2650 & $\mathrm{kgm}^{-3}$ \\
$\rho_{f}$ & 1000 & $\mathrm{kgm}^{-3}$ \\
$C_{r}$ & 800 & $\mathrm{Jkg}^{-1} \mathrm{~K}^{-1}$ \\
$C_{w}$ & 4200 & $\mathrm{Jkg}^{-1} \mathrm{~K}^{-1}$ \\
$\mu_{f}$ & 0.001 & $\mathrm{Nsm}^{-2}$ \\
$\lambda_{m m}$ & 2.9 & $\mathrm{Wm}^{-1} \mathrm{~K}^{-1}$ \\
$T_{r o}$ & 575 & $\mathrm{~K}^{-1}$ \\
$T_{D}$ & 150 & $\mathrm{~K}^{2}$ \\
$D^{\prime}$ & $1.0 \times 10^{-5}$ & $\mathrm{~m}^{2} \mathrm{day}^{-1}$ \\
$L_{f}$ & 1000 & $\mathrm{~m}$ \\
$\rho_{q}$ & 2650 & $\mathrm{kgm}^{-3}$ \\
$f_{q}$ & 0.2 & - \\
$\alpha$ & 0.47 & - \\
$C_{D}$ & $1.0 \times 10^{-5}$ & $\mathrm{~m}^{2} \mathrm{~s}^{-1}$ \\
$\mathrm{G}$ & 15,000 & $\mathrm{MPa}$ \\
\hline
\end{tabular}

In fracture aperture profile (in Figure 6.2), the rising limbs (positive slope) corresponds to an increase in fracture aperture resulting from dissolution, whereas the falling limbs (negative slope) represent a decrease in net fracture aperture resulting from dissolution followed by precipitation. This is because of the varying equilibrium concentration 
(Figure 6.3) and variation of reaction constant in the fracture with temperature (Figure $6.4)$.

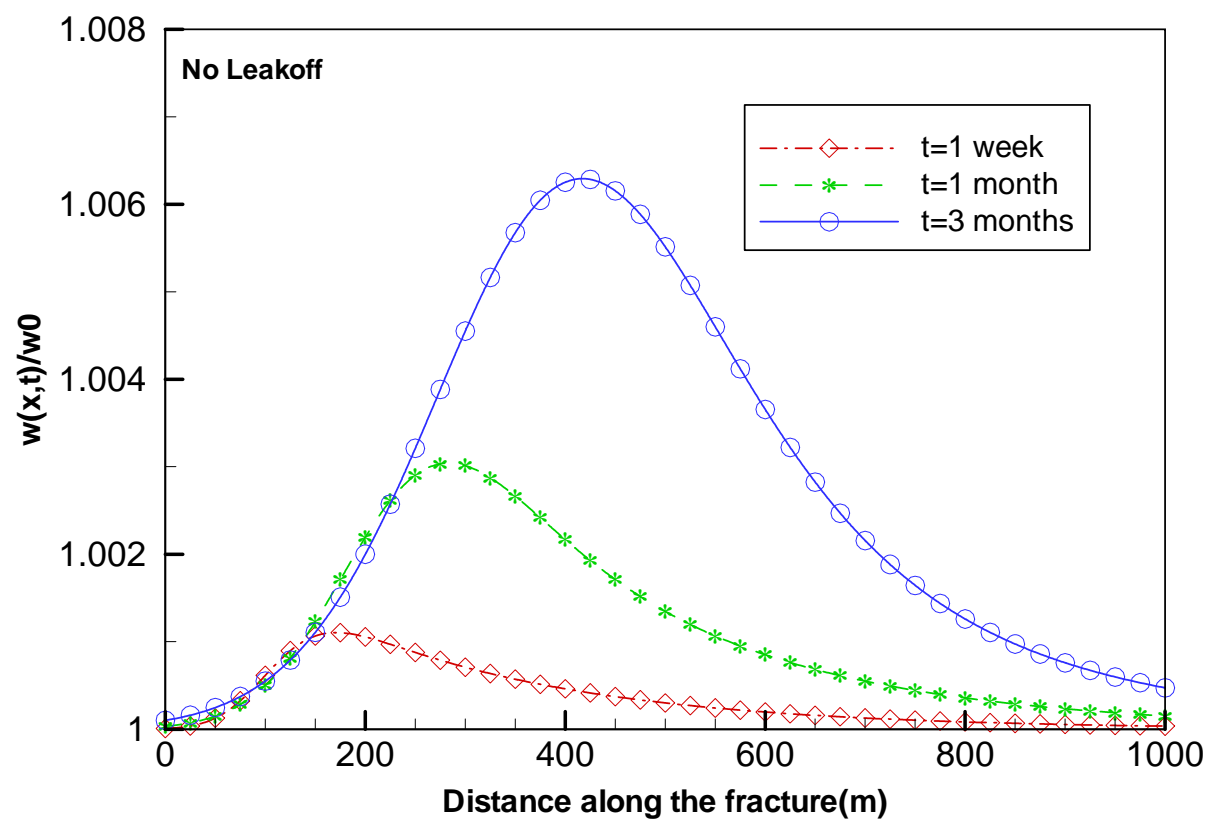

Figure 6.2. Normalized fracture aperture due to silica reactivity for different injection times (no leak-off).

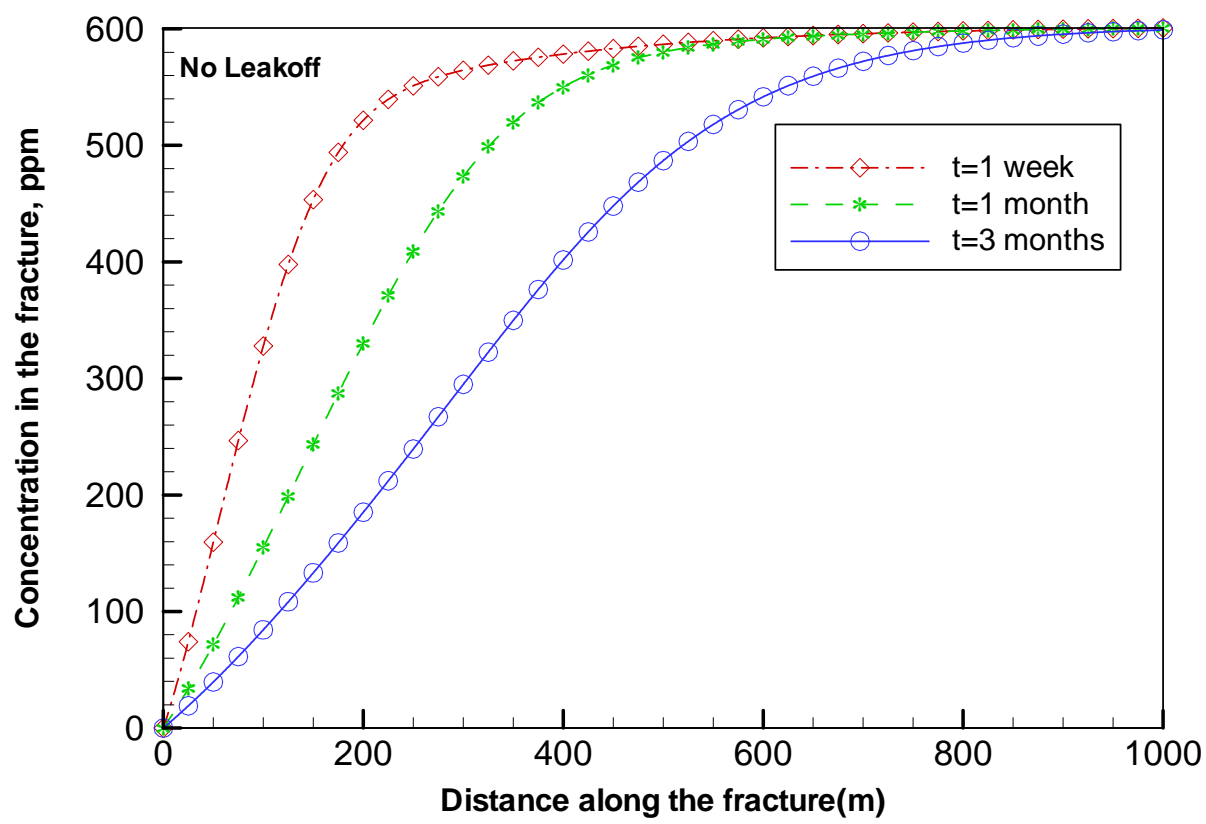

Figure 6.3. Concentration distribution in the fracture after 1 week, 1 month and 3 months when zero concentration fluid (water) is injected in the fracture. 
The corresponding silica concentration for the fracture aperture evolution (Figure 6.2) is depicted in Figure 6.3, where the injectate is pure water (zero concentration). It can be seen that the silica concentration increases within the fracture as the fluid moves away from the injection point. However, the concentration profile in the crack shows a reduction with time as it approaches the equilibrium concentration away from the injection point. This is because at early times, the injectate attains the rock matrix temperature (higher) at a shorter distance from the injection point. Moreover, in the diffusion dominated solute transport, the circulating fluid attains its equilibrium concentration close to the injection point for early injection time.

The influence of temperature on silica dissolution and precipitation can be described by observing the temperature profile in the fracture in (Figure 6.4). For the case considered, the temperature of the circulating fluid nearly reaches the rock temperature at $225 \mathrm{~m}, 500$ $\mathrm{m}$ and $850 \mathrm{~m}$ from the injection point for 1 week, 1 month and 3 months of injection times, respectively. As the heat is extracted from the formation over the fracture length, the rock is cooled to a lower temperature. Therefore, the fluid temperature profile in the fracture decreases with time affecting the dissolution/precipitation process in the crack.

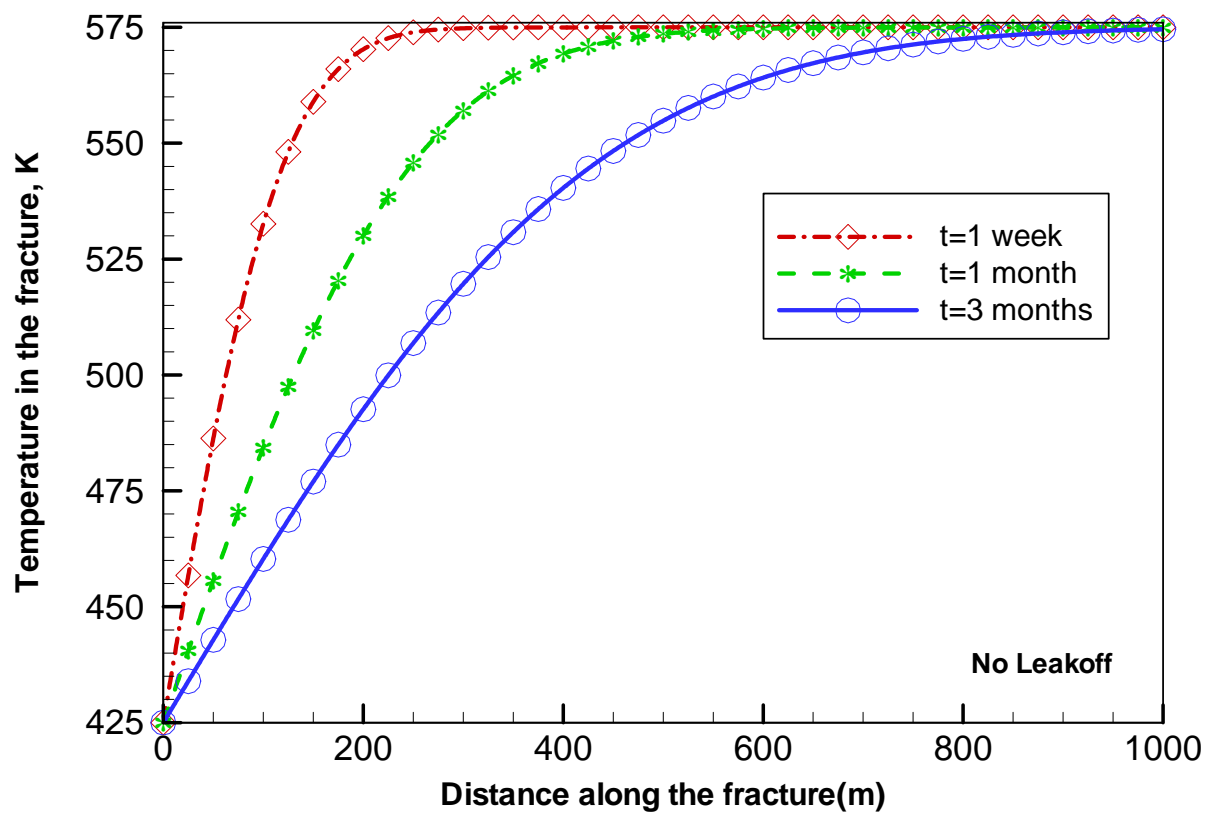

Figure 6.4. Temperature distribution in the fracture after 1 week, 1 month and 3 months. 


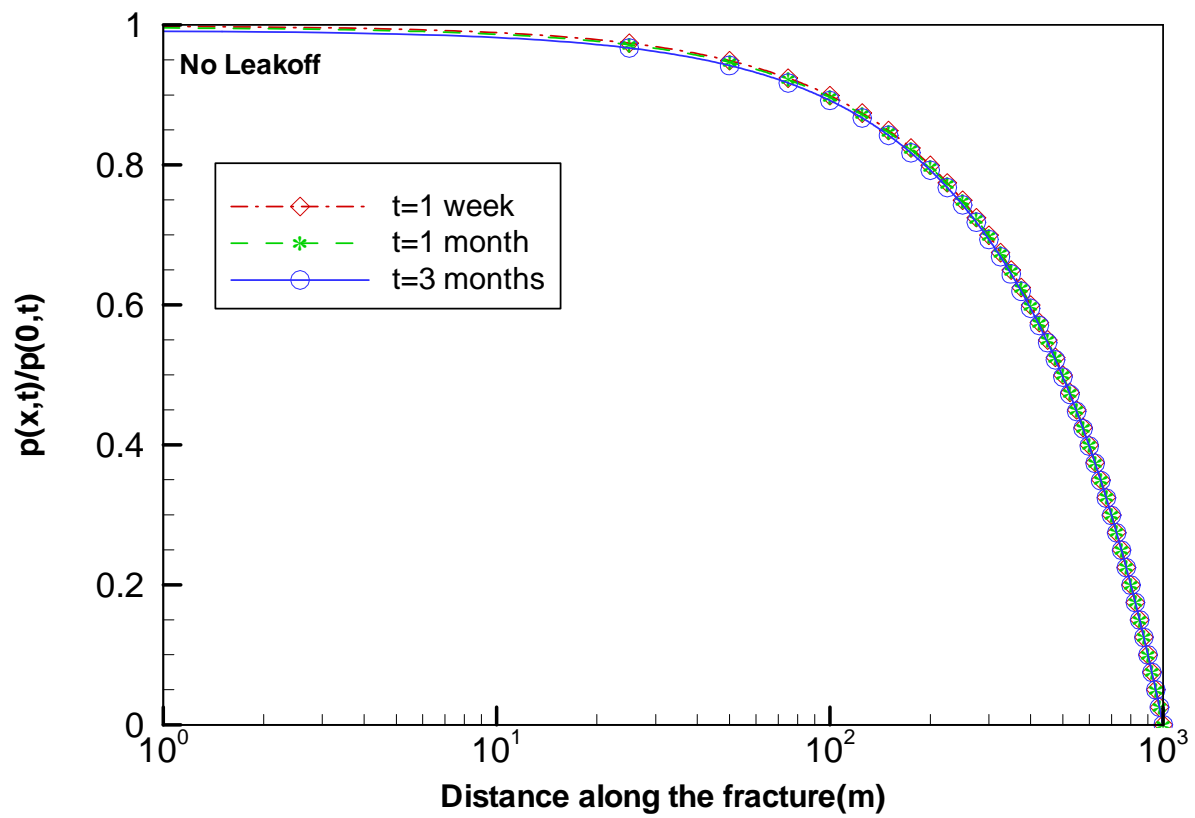

Figure 6.5. Normalized pressure profile in the fracture after 1 week, 1 month and 3 months.

In Figure 6.5, the normalized pressure profile corresponding to silica dissolution/precipitation along the fracture is shown for 1 week, 1 month and 3 months of injection time. The pressure is normalized with respect to the isothermal impermeable injection pressure, $\left(\mathrm{P}_{\mathrm{imp}}(0, \mathrm{t})=1.2 \mathrm{MPa}\right)$. As in this case, the fracture aperture is not influenced by an appreciable amount due to silica dissolution/precipitation, the pressure profiles for various injection times differ only by a small amount.

Fluid leak-off into the formation influences the dissolution/precipitation of silica in the fracture. To see the effects of fluid leak-off on the silica dissolution/precipitation, consider the results of under-saturated and supersaturated fluid injections as shown in Figure 6.6 and Figure 6.7. The fracture aperture profile corresponding to silica dissolution/precipitation by pure water injection for 1 week and 3 months are shown in Figure 6.6 for the cases of leak-off and no leak-off. Note that fluid leak-off tends to accelerate dissolution up to about half the fracture length. Precipitation is also accelerated but at longer injection times. Essentially the curve attains a higher peak and shifts to the left with increased leak-off. This pattern of acceleration/deceleration is observed at short times (i.e. of 1 week) but at a lower magnitude. Thus, the leak-off enhances both dissolution and precipitation in under-saturated case because more silica mass is exchanged in between fracture and rock-matrix. 


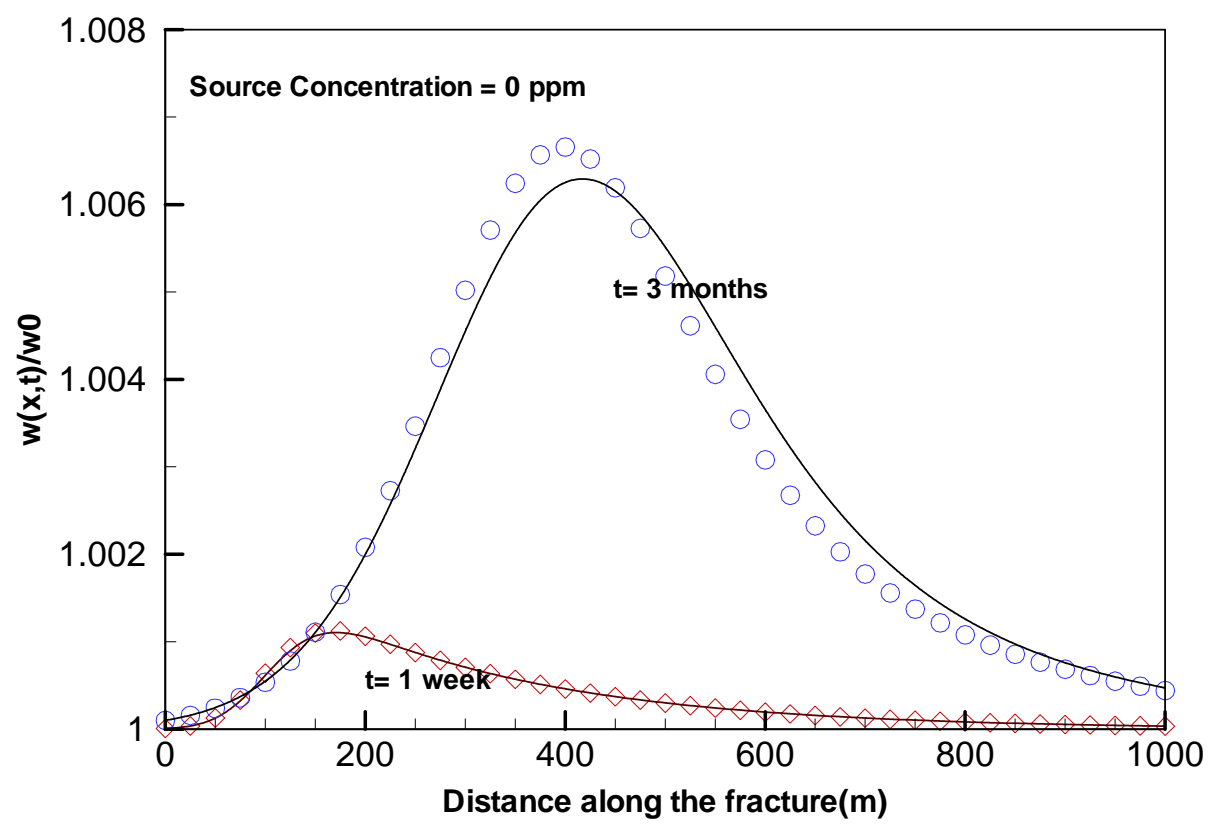

Figure 6.6. Normalized fracture aperture due to silica dissolution/precipitation at 1 week and 3 months (under-saturated case; Solid lines: No leak-off: and symbols: $m=0.5$ ).

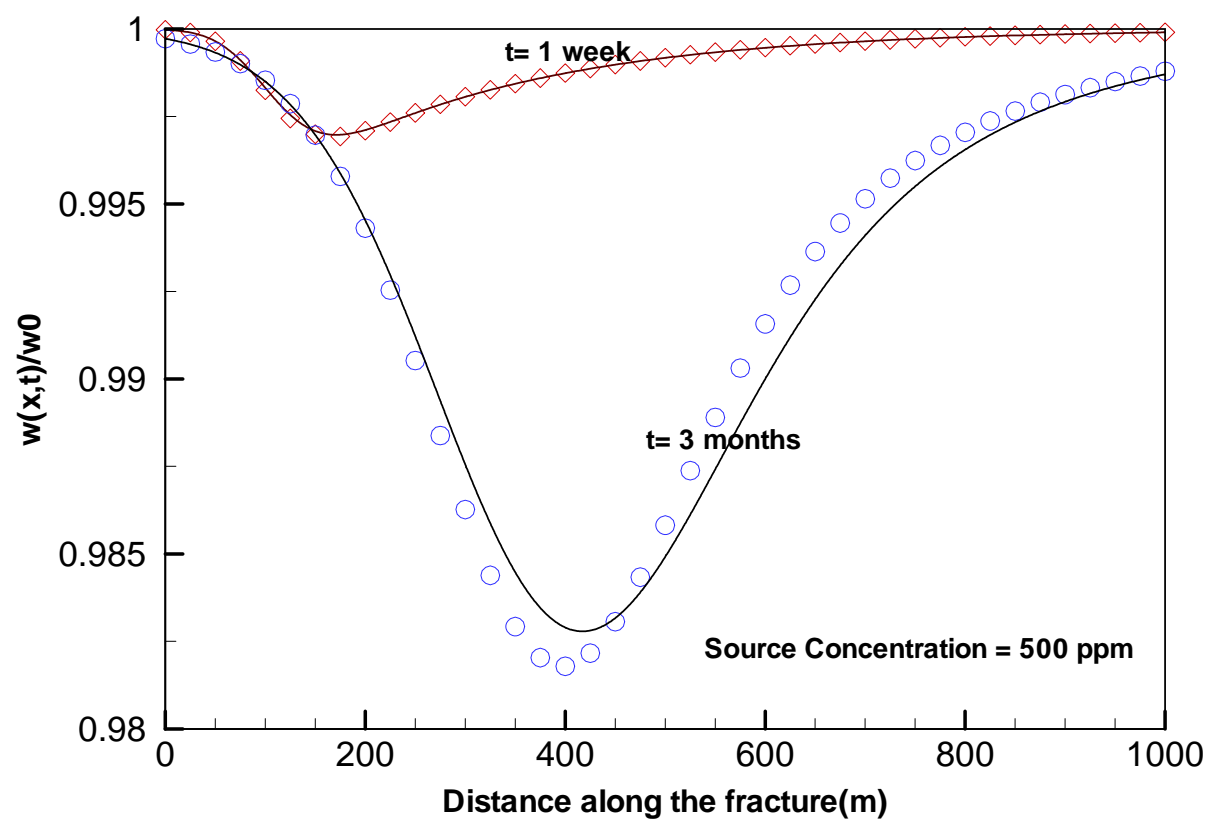

Figure 6.7. Normalized fracture aperture due to silica dissolution/precipitation at 1 week and 3 months (super-saturated case; Solid lines: No leak-off: and symbols: $m=0.5$ ).

On the other hand, if supersaturated fluid of $500 \mathrm{ppm}$ is injected in the fracture, the aperture decreases appreciably with longer injection time (Figure 6.7). This effect is enhanced by leak-off and the peak aperture reduction shifts away closer to the injection 
point. This is because the injectate in the fracture attains its equilibrium stat at a smaller distance in view of a temperature dependent of temperature reaction rate.

Figure 6.8-6.10 show the normalized fracture aperture resulting from poroelastic and thermoelastic deformation in the presence of fluid leak-off, respectively. In Figure 6.8, the normalized fracture aperture is plotted against distance along fracture for various values of leak-off ratio (m) after 3 months of injection time. It can be observed that the fracture closure is relatively small and evolves slowly for lower levels of leak-off. The maximum reduction of fracture aperture occurs at the injection point where the induced pressure $\mathrm{p}(\mathrm{x}, \mathrm{t})$ and hence leak-off is the greatest.

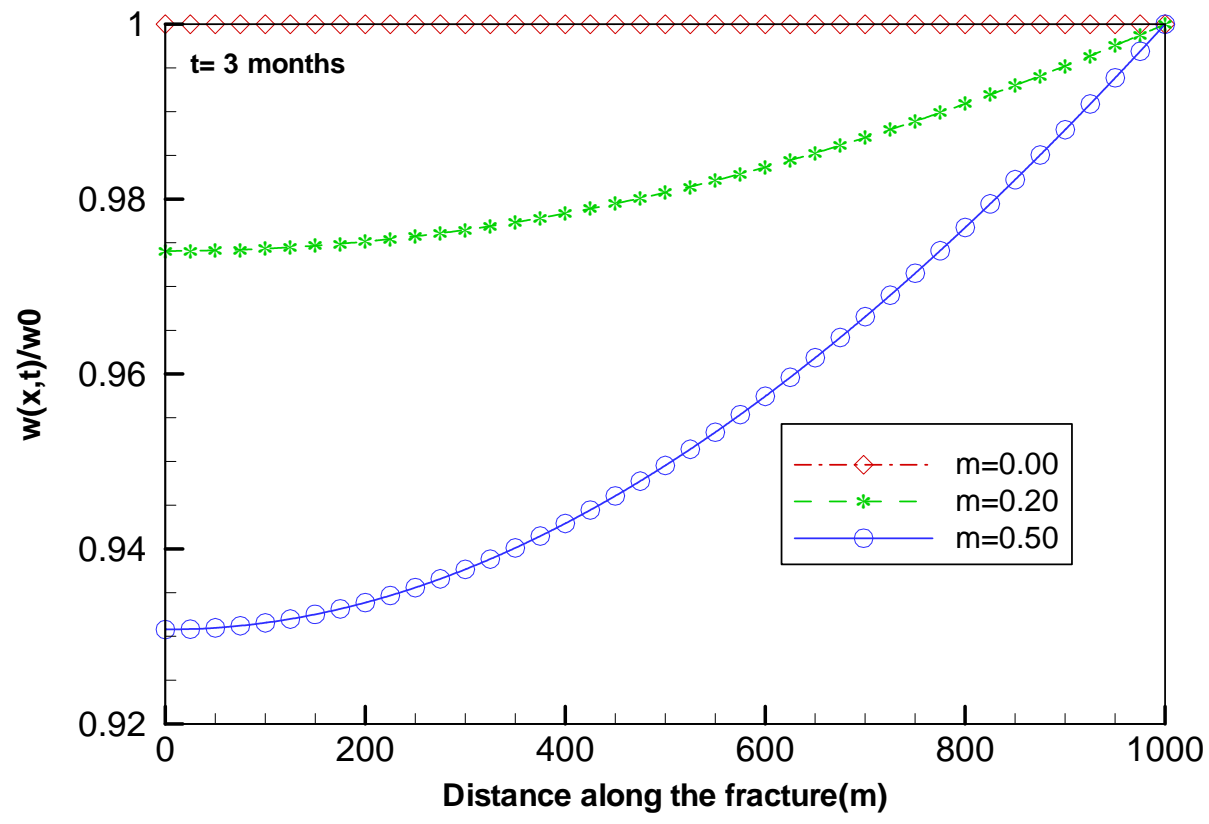

Figure 6.8. Normalized fracture aperture due to influence of poroelastic stress on fracture aperture after 3 months. The figure is drawn for different fluid loss/injection ratios $(\mathrm{m})$.

Figure 6.9 and 6.10 show the normalized fracture aperture history at the injection point for poroelastic and thermoelastic cases, respectively. The aperture profile has same shape in both plots, but the effects are opposite. Figure 6.9 shows that the fracture aperture change due to poroelastic effects is pronounced with higher fluid leak-off $(m>0)$ and at early time. However in the thermoelastic case, the fracture aperture at the injection point becomes larger at a later time when compared to the impermeable case $(m=0)$ as shown in Figure 6.10. This is because leak-off increases the fluid residence time and the heat having been extracted from the rock but this effect evolves very slowly. 


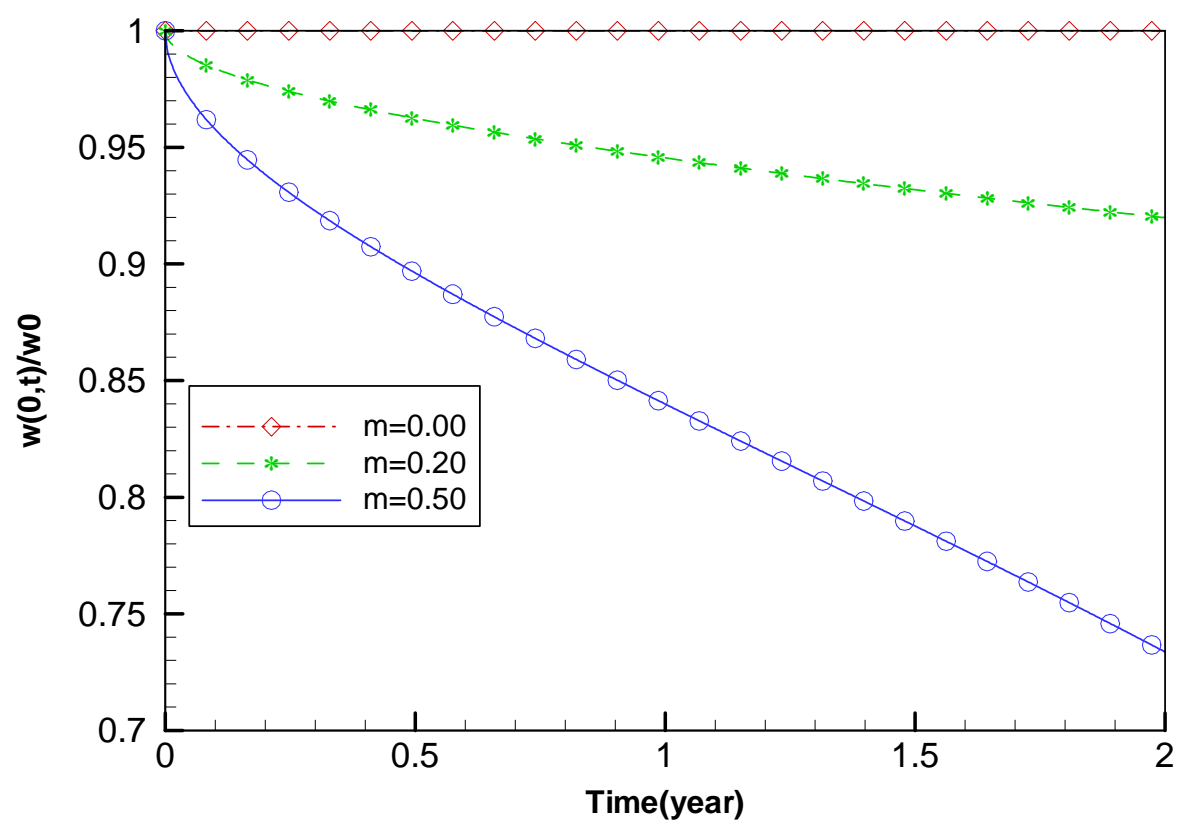

Figure 6.9. Normalized fracture aperture at the injection point due to influence of poroelastic stress on fracture aperture for different fluid loss/injection ratios $(m)$ and injection times.

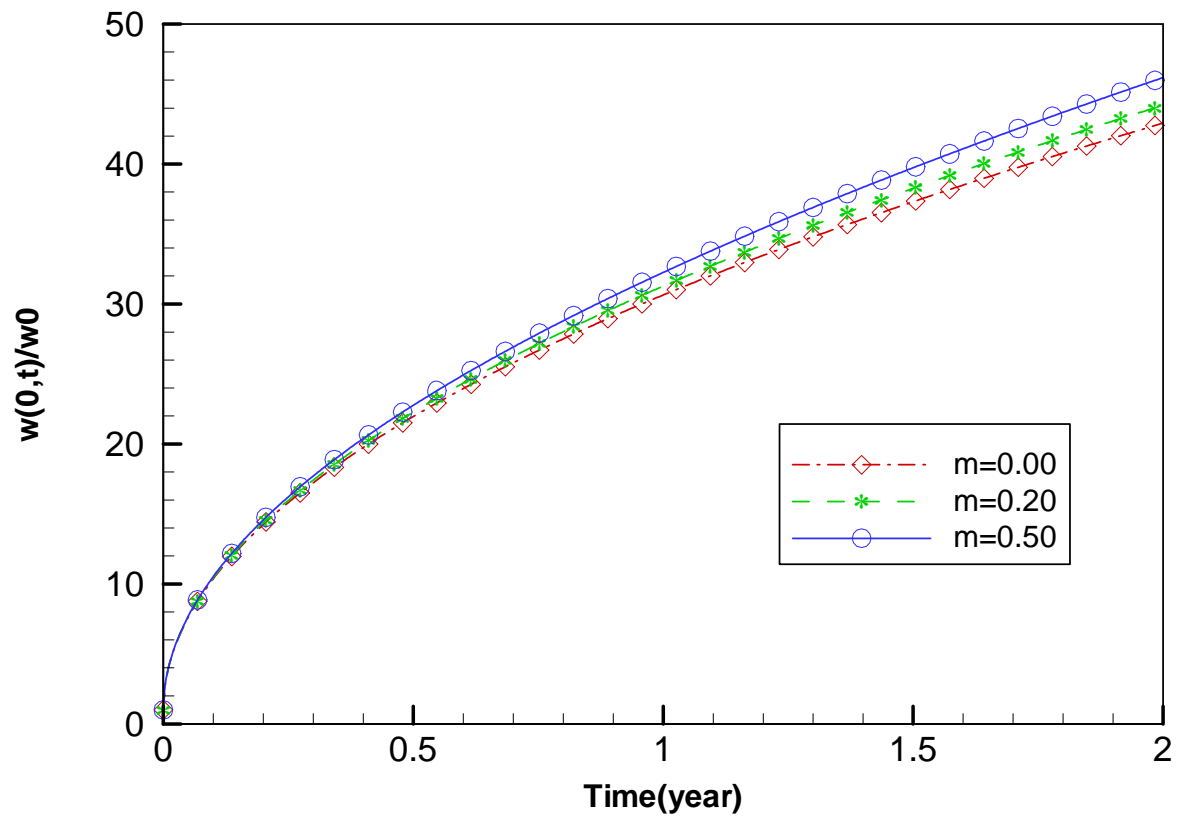

Figure 6.10. Normalized fracture aperture at the injection point due to thermoelastic effects for different fluid loss/injection ratios $(m)$ and injection times.

Figure 6.11 shows induced pressure distribution in the fracture corresponding to the poroelastic effects (as illustrated in Figure 6.8) and thermoelastic effects after 3 months of injection time; the pressure is normalized with respect to the isothermal impermeable injection pressure of $\left(\mathrm{P}_{\text {imp }}(0, \mathrm{t})=1.2 \mathrm{MPa}\right)$. It can be seen that the pressure profiles in the poroelastic case have higher magnitudes compared to those of thermoelastic case 
(because of the larger poroelastic width contraction). The poroelastic and thermoelastic pressure profiles are nearly identical near the extraction point as the poroelastic fracture aperture change becomes negligible (see Figure 6.8) in response to pressure reduction in the fracture. Pressure profiles in the thermoelastic case change their slope near half fracture length where the thermoelastic stresses in the fracture are largely reduced.

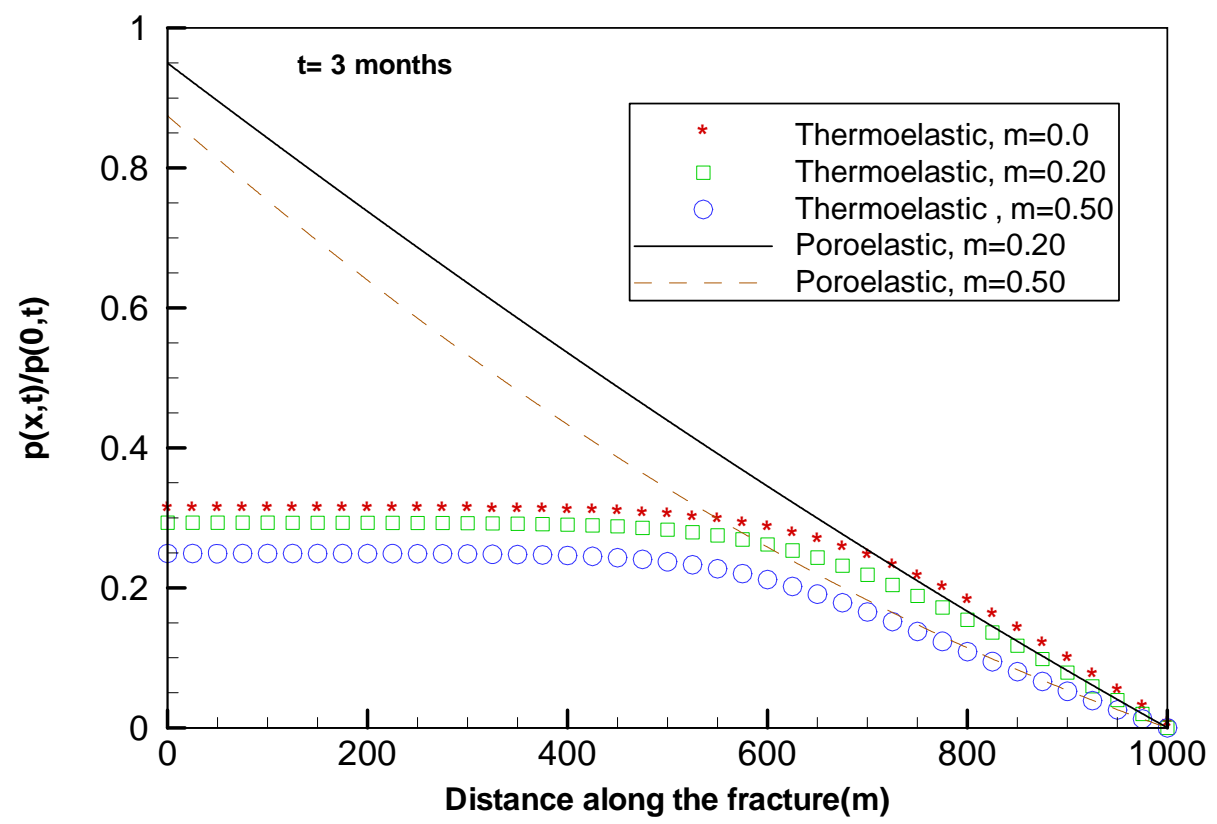

Figure 6.11. Normalized pressure profile along he fracture for different fluid loss/injection ratios

$(m)$ after 3 months of injection. Lines: poroelastic case and symbols: thermoelastic case.

We have analyzed reactive chemistry and poro-thermoelastic effects of low-temperature fluid injection in a natural fracture using semi-analytical poro-thermo-chemo model. The governing equations of the model were solved analytically (but some requires numerical inversion) to investigate fracture aperture change caused by low temperature fluid injection and fluid leak-off into the formation. The corresponding pressure profiles were also calculated. Both the solute reactivity along the fracture and its diffusion into the rock-matrix are considered using temperature depended reaction kinetics for a single component (silica system). The results indicate that the circulating fluid concentration attains its saturation farther away from the injection point for longer injection times. The under-saturated fluid injectate has tendency to widen up the aperture however supersaturated fluid leads to fracture closure. Similarly fluid leak-off can influence the silica dissolution/precipitation with considerable amount after longer injection time. The thermoelastic effects are dominant near the injection point. Although fluid leak-off does not change the fracture aperture significantly, it can lead to pore-pressure increase. 


\section{References}

1. Bolton, E.W., A. C .Lasaga, and D. M. Rye, 1996. A model for the kinetic control of quartz dissolution and precipitation in porous media flow with spatially variable permeability: Formulation and examples of thermal convection. J. Geophys. Res., 101, 22157- 22187.

2. Lowell, R.P., P. Van Cappellen and L.N. Germanovich, 1993. Silica precipitation in fractures and the evolution of permeability in hydrothermal upflow zones. Science, 260, 192-194.

3. Kumar, S., and A. Ghassemi, 2005. Numerical modeling of non-isothermal quartz dissolution and precipitation in a coupled fracture-matrix system. Geothermics, 34, 411439.

4. Carroll S., E. Mroczek, M. Alai, and M. Ebert, 1998. Amorphous silica precipitation (60 to 120 degrees C): Comparison of laboratory and field rates. Geochim Cosmochim Acta, 62, 1379-1396.

5. Johnson, J.W., K.G. Knauss, W.E. Glassley, L.D.DeLoach, and A.F.B.Tompson, 1998. Reactive Transport Modeling of Plug-Flow Reactor Experiments: Quartz and Tuff Dissolution at $240^{\circ}$ C. J. Hydrol. 209, 81-111.

6. Dobson, P. F., T. J. Kneafsey, E. I .Sonnenthal, N. Spycher, and Apps, J.A., 2003. Experimental and numerical simulation of dissolution and precipitation: implications for fracture sealing at Yucca Mountain, Nevada. J. Contam. Hydrol., 62-63, 459-476.

7. Pruess, K. (1991). TOUGH2: A general numerical simulator for multiphase fluid and heat flow. Lawrence Berkeley Laboratory Report LBL-29400, Berkeley, California.

8. Xu, T., and Pruess, K. (1998), Coupled modeling of nonisothermal multiphase flow, solute transport and reactive chemistry in porous and fractured media: 1 . Model development and validation. Lawrence Berkeley National Laboratory Report LBNL42050, Berkeley, California, 38 pp.

9. $\mathrm{Xu}, \mathrm{T}$., and K. Pruess. (2001) Modeling multiphase nonisothermal fluid flow and reactive geochemical transport in variably saturated fractured rocks: 1. Methodology, Am. J. Sci., 301, 16-33.

10. Wells, J. T., Ghiorso, M. R., 1991. Coupled fluid flow and reaction in mid-ocean ridge hydrothermal systems: the behavior of silica. Geochim. Cosmochim. Acta, 55(9), 24672481.

11. Rimstidt, J.D., and Barnes, H.L., 1980. The kinetics of silica-water reactions. Geochim. Cosmochim. Acta, 44, 1683-1699.

12. Arnorsson, S., Gudmundsson, B. T., 2003. Geochemical monitoring of the response of geothermal reservoirs to production load - examples from Krafla, Iceland. Proc. Int. Geothermal Conf., Reykjavik, Sept. 2003, pp. 30-36; SO7 Paper117. 
13. Manning, C.E., 1994. The solubility of quartz in H2O in the lower crust and upper mantle. Geochimica et Cosmochimica Acta, 58, 4831- 4839.

14. Yariv, S., Cross, H., 1979. Geochemistry of volloid systems for Earth scientists. Berlin: Springer-Verlag, $450 \mathrm{p}$.

15. Zhou, X., Ghassemi, A., and Cheng, A.H.-D. 2009. A Three-Dimensional Integral Equation Model for Calculating Poro- and Thermoelastic Stresses Induced by Cold Water Injection into a Geothermal Reservoir. Int. J. Num. Anal. Methods Geomech. DOI:10.1002/nag.

16. Cheng, A.H.-D., A. Ghassemi and Detournay, E. 2001. A two-dimensional solution for heat extraction from a fracture in hot dry rock. Int. J. Numer. Analyt. Meth. Geomech, 25, 1327-1338.

17. Tang D.H., Frind E.O. and Sudicky E.A. 1981. Contaminant transport in fractured porous media: analytical solution for a single fracture. Water Res. Research, 17-3, 555-564.

18. Steefel Carl I. and Lichtner Peter C. 1998. Multicomponent reactive transport in discreate fractures: I. Controls on reaction front geometry. J. Hydrology, 209, 186-199.

19. Wangen M., and Munz Ingrid A. 2004. Formation of quartz veins by local dissolution and transport of silica, Chemical Geology, 209, 179-192.

20. Ghassemi, A., and Kumar, S. 2005. Numerical modeling of non-isothermal quartz dissolution/precipitation in a coupled fracture-matrix system. Geothermics, 34, 411-439.

21. Robinson, B.A., and Pendergrass, J. 1989. A combined heat transfer and quartz dissolution/deposition model for a hot dry rock geothermal reservoir. Proc. 14th Workshop Geothermal Reservoir Engineering, Stanford University, Jan 24-26, 207-212.

22. Ghassemi, A., Tarasovs, A. and Cheng, A.D.-H. 2005. Integral equation solution of heat extraction induced thermal stress in enhanced geothermal reservoirs. Int. J. Num. \& Anal. Methods in Geomechanics, 29, 829-844.

23. Steefel, C.I., Litchner, P.C. 1998. Multicomponent reactive transport in discrete fractures: I. Controls on reaction front geometry: J. Hydrology, 209, 186-199.

24. Stehfast, H., 1970. Numerical inversion of Laplace transforms. Comm. ACM, 13, 47-49.

25. Gringarten, A.C., Witherspoon, P.A. and Ohnishi, Y. 1974. Theory of heat extraction from fracture hot dry rock. J. Geophys. Res., 80, 1120-1124, 1974.

26. Ghassemi, A., Nygren, A., Cheng, A.D.-H. 2008. Effects of heat extraction on fracture aperture: A poro-thermoelastic analysis. Geothermics, 37 (5), 525-539. 


\section{Conclusions \& Future Research}

The role of poro-thermo-mechanical processes in rock failure and fracturing, and permeability change were investigated through development and implementation of porothermoelastic displacement discontinuity boundary element methods, and coupled geochemical models.

A number of applications and analyses were presented to highlight the impact of various mechanisms. In particular, fracture opening and slip, injection/production in naturally fractured reservoir, and fracture propagation were considered and 3D fracture slip was studied. Finally, examples were presented to illustrate the combined effects of silica dissolution/precipitation and poro-thermoelastic processes on the fracture aperture and pressure distributions during circulation operations.

\subsection{Injection into Fractured Reservoir, Fracture Propagation and Permeability Change}

The opening of a crack under combined poro-thermomechanical was considered under geothermal conditions and very small and very large times. It was found that the poroelastic effects on fracture opening are generally small for granitic rocks. This poroelastic mechanism corresponding to fluid diffusion from a fracture into the rock tends to close the fracture with time i.e., the width of the crack decreases. This is because the fluid injected into the fracture diffuses into the porous formation, increasing the pore pressure around the crack which, in turn, induces a dilatation of the rock formation. This increased pore pressure, however, can cause rock failure in the vicinity of the main fracture and contribute to overall permeability enhancement.

The thermoelastic effect causes the crack to open gradually and in a much slower pace compared to the poroelastic effect. The crack opens as a result of the shrinking of the rock formation. The rock shrinking can also cause additional tensile fractures.

By comparing the temporal variations of fracture opening due to stress, pore pressure, and thermoelastic loading, it is concluded that in many practical situations in EGS development, modes 1 and 2 contribute to fracture propagation during conventional hydraulic fracturing jobs. However, during injection operations their effect can become negligible in comparison to that of cooling. This is evident from the results showing that the contribution of mode 3 or thermal stress is much higher in stimulation and circulation operations which are conducted over a long period of time. For example, for rock types of Coso, a $200{ }^{0} \mathrm{C}$ cooling for a time of $t=10^{6} \mathrm{sec}$, the cooling-induced opening is approximately 2.5 times that of the instantaneous crack opening in response to the applied hydraulic pressure of $15 \mathrm{MPa}$. 
By developing and implementing a joint model for the poro-thermoelastic DD methods, a fractured reservoir simulator has been developed that allows investigation of the reservoir response to injection and extraction operations while considering natural fracture slip and opening. Preliminary applications explicitly show the previously described poroelastic and thermoelastic phenomena in the context of reservoir engineering. In particular, permeability change and variation of pressure at the injection and production wells are clearly observed. The results provide an explanation for the phenomenon noted during water injection in well number 83-16 in Coso, i.e., injectivity varies water temperature; injecting cold water decreases injection pressure and subsequent hot water injection increases it.

Continued fluid injection tends to increase the pore pressure inside the rock thus reducing the crack width to some extent by Mode 2 or poroelastic effects. However, as noted previously, cooling induces large tensile stresses in the rock, thus creating new cracks and increasing the width of existing ones. If the pre-existing crack surfaces are continuously cooled, the major portion of the influence of cooling tends to materialize within a week of injection for the rock properties considered. This mechanism is reversed once the well is put on hot water injection. Heating tends to reduce the fracture widths and tends to close some of the newly opened fractures. Furthermore, continued hot water injection will lead to additional poroelastic effects which also contribute to crack closure.

The fracture propagation work included development and implementation of a new fracture propagation criterion in a partially-coupled DD code. An extension of the Neuber-Novozhilov structural fracture propagation criterion was used for mode I (tensile) and mode II (shear) propagation under compressive loads. In addition to allowing numerical simulation of crack growth, the criterion can model change of propagation mode, crack branching, and coalescence. The results of example simulations illustrated the importance of the role of Mode 2 loading (pore pressure gradient) in fracture propagation. The diffusion of pore pressure inside the crack into the rock can lead to change of possibility, mode and direction of fracture propagation. In most cases, besides fracture growth speed, rock and fluid properties, fracture geometry, in-situ stresses and internal fluid pressure all play an important role in determining the fracture propagation behavior in a permeable poroelastic medium.

\subsection{Three-Dimensional Thermoelastic Effects}

The two-dimensional plane strain crack model is only an approximation of the threedimensional crack opening and propagation. In fact, the two-dimensional model tends to significantly overestimate the fracture width due to cooling (by 57\%). Therefore, a 3D model was developed in the time domain to calculate poroelastic and thermally-induced stresses associated with injection and extraction. 
This was achieved using a 3D integral equation formulation to model heat extraction from a planar fracture in an infinite reservoir and to calculate the temperature and heat flux distributions within the crack and the matrix. This model was then used to determine the 3D thermal stresses resulting from cold water injection, and to estimate the natural fracture slip using a Mohr-Coulomb joint model. The approach used is distinguished from existing analytical and numerical works that treat the heat conduction in the geothermal reservoir as one-dimensional and perpendicular to the fracture. The analytical procedures and computational schemes were tested by considering the problem of injection into an infinite fracture and comparing the results with a semi-analytical solution. Then, using data from Coso, the thermally induced stresses were calculated for the injection experiment in Well 83-16. It has been found that the normal (axial) stress on the fracture surface is smaller than that predicted by uniform cooling of the crack surface. Also, the normal stress is tensile up to some distance from the injection well, its maximum occurs near the injection point and gradually approaches zero away from it. The normal stress becomes compressive at some distance away from the cooled region because as the cooled rock shrinks, it tends to pull on the exterior rock material (strain compatibility) inducing a compressive stress in it. It was interesting to note that the induced tensile stress near the injection well causes the rock to contract causing the aperture to open wider. Then, for a constant injection rate, the injection pressure drops due to the increased conductivity. This is indeed observed in Well 83-16 of the Coso. The explicit consideration of the fracture provides more insight for this mechanism and was subsequently considered.

In considering a 3D fracture, the model was first applied to study the impact of poroelasticity on the fracture opening and slip. It was found that the application of a normal stress loading on the fracture surfaces increases the fracture opening with time because of the dissipation of the pore pressures in the rock. On the other hand, a pore pressure loading causes fracture closure as fluid leaks-off from the fracture into the rock matrix. This is consistent with our previous 2D studies. Simulations of injection into a critically stressed fracture at pressures insufficient to jack it open showed that the fracture slips and dilates. Thereafter, the fracture slip decreases as the matrix dilates in response to pore pressure diffusion, increasing the normal stress on the fracture surface and reducing the crack opening. This transient slip can contribute to the injection pressure variations as well as to induced reservoir seismicity observed in enhanced or engineered geothermal systems

Consideration of fracture slip, opening and injection pressure variation in response to injection/extraction clearly showed that the fracture pressure and aperture are predominantly affected by the poroelastic effects at the early stage and by the thermoelastic effect after a long time of fluid injection. When the initial fracture aperture is assumed to be small enough to generate a relatively large fracture pressure compared to the initial reservoir pressure, the large fluid leakoff decreases the fracture pressure and aperture at early stages when compared to the case of no fluid leakoff. However, the leakoff influence becomes less evident for longer injection times. After a long time of fluid injection, the fracture aperture increases significantly, mainly due to the thermoelastic effect. 


\subsection{Influence of Quartz Precipitation and Dissolution on Fracture Permeability}

An analytical model was developed to simulate the combined effect of poro-mechanical, thermal, and reactive solute transport (with quartz dissolution/deposition kinetic model) in a fracture-matrix system. Both the solute reactivity along the fracture and its diffusion into the rock-matrix were considered using temperature depended reaction kinetics for a single component (silica system). The solution of the governing equations was used to investigate the fracture aperture change caused by low temperature fluid injection and fluid leak-off into the formation. The corresponding pressure profiles were also calculated. Simulations were carried out to analyze the effects of reactive chemistry and poro-thermoelasticity when injecting into a natural fracture under the conditions of Coso geothermal reservoir. The results indicated that the circulating fluid concentration attains its saturation further away from the injection point for longer injection times. An undersaturated fluid injectate has a tendency to widen the aperture, while a supersaturated fluid leads to fracture closure. Similarly, fluid leak-off can influence the silica dissolution/precipitation to a considerable degree after longer injection times. The thermoelastic effects are dominant near the injection point. Although fluid leak-off does not significantly change the fracture aperture, it can lead to pore-pressure increase.

\subsection{Future Work}

The fracture propagation and coalescence algorithms need to be coupled to fluid flow calculation to allow one to calculate the pressure necessary to extend a fracture into the reservoir. The full coupling between heat transfer and fluid flow should be considered and the capability of propagation in the presence of natural fractures added.

The reactive transport component should be added to the 2D naturally fractured reservoir simulator to take into account the influence of porothermal stresses and silica reactivity on permeability evolution in a coupled manner. In addition, it will be of interest to consider a multi-component chemical system. This can be important as the net result of the process of precipitation/dissolution of various minerals in the reservoir matrix influence the amount of exposed fracture surfaces (fracture walls) and subsequently affect fluid circulation in the system.

The 3D fracture algorithms should be improved to efficiently model multiple fracture systems. Also, addition of reactive transport models would be useful in understanding the impact of non-isothermal reactive transport using a geometrically realistic fracture model. 


\section{PUBLICATIONS \& Technology Transfer/ Student Training}

The research activities have trained a number of graduate students in the field of Rock Mechanics for geothermal reservoir development namely, Andrew Nygren, Jun Ge, Qingfeng Tao, Chakra Rawal, Wenxu Xue, and Xiaoxian Zhou. Mr. Andrew Nygren was awarded the best M.S. Thesis Award by the American Rock Mechanics Association in 2006. These students have participated in preparing publications and making presentation in national/international conferences. We have published several papers, and have 3 additional manuscripts under review:

1. Ghassemi, A., Nygren, A., Cheng, A.D.-H. 2008. Effects of heat extraction on fracture aperture: A poro-thermoelastic analysis. Geothermics, 37 (5), 525-539.

2. Zhou, X., Ghassemi, A., and Cheng, A.H.-D. 2009. A Three-Dimensional Integral Equation Model for Calculating Poro- and Thermoelastic Stresses Induced by Cold Water Injection into a Geothermal Reservoir. Int. J. Num. Anal. Methods Geomech. DOI:10.1002/nag.

\section{Papers under Review}

3. Ghassemi, A., X. Zhou. 2009a. A Three-dimensional poroelastic displacement discontinuity method for simulating hydraulic and natural fractures. It. J. Rock Mech. Min. Sci.

4. Ghassemi, A., X. Zhou. 2009b. Simulation of aperture and pressure variation caused by poro- and thermoelastic effects of injection into a geothermal reservoir. It. J. Rock Mech. Min. Sci.

5. Ghassemi, A. Rawal, C. 2008. Reactive silica transport in hot poroelastic rock and its effects on fracture aperture. Geothermics.

\section{Conference Papers}

6. Xue, W., Ghassemi, A., 2009. Poroelastic Analysis of Hydraulic Fracture Propagation. 43rd US Rock Mech. Symp., Asheville, NC June 28th - July 1, 2009.

7. Zhou, A., Ghassemi, A. 2009. Three-dimensional poroelastic displacement discontinuity simulation of natural fractures. 43rd US Rock Mechanics Symposium and 4th U.S.-Canada Rock Mechanics Symposium, held in Asheville, NC June 28th - July 1, 2009.

8. Ghassemi, A., 2009. Rock joints \& fractures in geothermal \& petroleum reservoir development: poro-thermoelastic effects. International Conference on Rock Joints and Jointed Rock Masses, Tucson, AZ, Jan. 2009.

9. Zhou, A., Ghassemi, A. 2009. Three-Dimensional Poroelastic Simulation of Hydraulic and Natural Fractures Using the Displacement Discontinuity Method. 34th Stanford Geothermal Workshop, Feb. 9-11th, Palo Alto. 
10. Rawal, C., Ghassemi, A. 2008. Fracture aperture change in response to reactive transport of silica and thermoelastic effects. Proc. 42nd U.S. Rock Mech. Symp., June 29 - July 2, San Francisco.

11. Zhou, X., Ghassemi, A. 2008. A three-dimensional model for calculating poroand thermoelastic Stresses Induced by Cold Water Injection into a Geothermal Reservoir. Proc. 42nd U.S. Rock Mech. Symp., June 29 - July 2, San Francisco.

12. Zhou, X., Ghassemi, A. 2008. A three-dimensional poroelastic model for water injection into a geothermal reservoir. 33rd Stanford Geothermal Workshop of Reservoir Engineering.

13. Ge, J., Ghassemi, A. 2007. Pore pressure and stress distributions around an 14. injection-induced fracture. Transactions, Geothermal Res. Coun., Reno.

15. Nygren, A. and Ghassemi, A. 2006. Coupled Poroelastic and Thermoelastic Effects of Injection into a Geothermal Reservoir. 40th U.S. Rock Mech. Symp., Golden, CO. 